
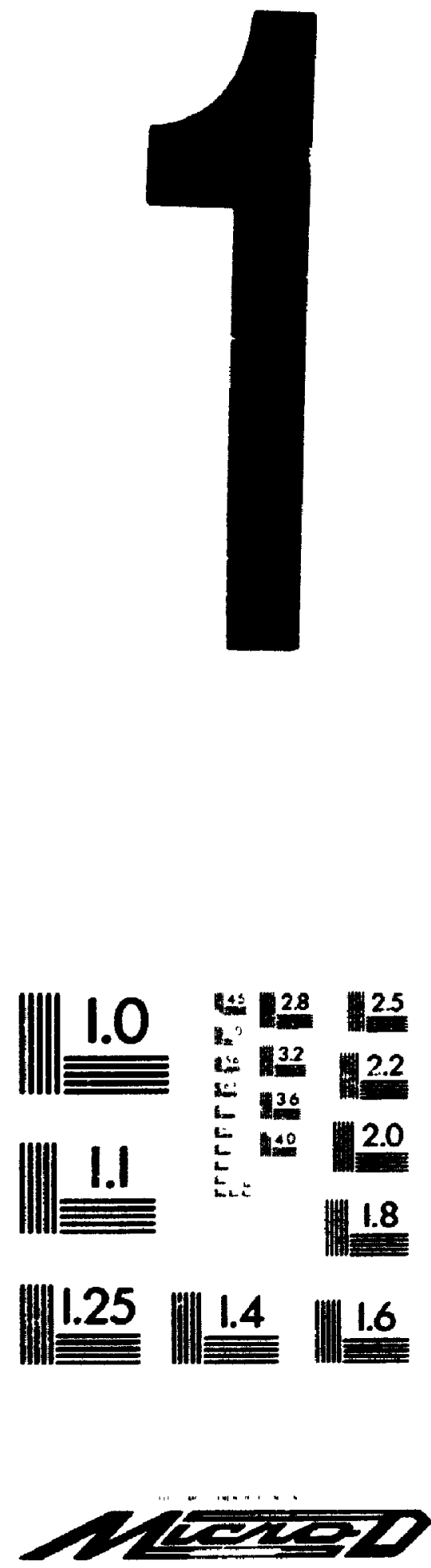
Orema, Ceneda

KTA ON4

NOTICE

The quality of this microform is heavily dependent upon the quality of the original thesis submitted for microfilming. Every effort has been made to ensure the highest quality of reproduction possible.

If pages are missing, contact the university which granted the degree.

Some pages may have indistinct print especially if the original pages were typed with a poor typewriter ribbon or if the university sent us an inferior photocopy.

Peproduction in full or in part of this microform is governed by the Canadian Copyright ACt, R.S.C. 1970, C. C-30, and subsequent amendments.

\begin{abstract}
AVIS
La qualité de cette mirsolorme dépend grandement de la qualité de la these soumise au microtilmage. Nous avons tout fait pour assurer une qualites superieure de reproduc. tion.

S'il manque des pages, veuillaz communiquer avec luniversite qui a contéré le grade.

La qualité d'impression de certaines pages peut laisser á désirer, surtout si les pages originales ont été dactylographiées a raide d'un ruban usé ou si l'université nous a fail parvenir une photocopie de qualité inférieure.

La reproduction, méme partielle, de cette microforme es soumise a la Loi canadienne sur le droil d'auteur. SRQ 1970, c. C-30, et ses amendements subséquents.
\end{abstract}




\title{
ANALYSIS OF THE APPLICATION OF TRELLIS CODING TO \\ SPREAD SPECTRUM MULTIPLE ACCESS SYSTEMS
}

\author{
by \\ GARY D. BOUDREAU \\ A thesis submitted to \\ the Faculty of Graduate Studies and Research \\ in partial fulfillment of \\ the requirements for the degree of
}

Doctor of Philosophy

Department of Systems and Computer Engineering

Carleton University

Ottawa, Ontario

October 11, 1989

Ccopyright

1989, Gary D. Boudreau 
National Library

of Canada

Canadian Theses Service

Ottawa. Canoda

KIA ONA
Bibliotheque nationale

du Canada

Service des theses canadiennes

The author has granted an irrevocable nonexclusive licence allowing the National Library of Canada to reproduce, loen, distibute or sell copies of his/her thesis by any means and in any form or format, making this thesis available to interested persons.

The author retains ownership of the copyright in his/her thesis. Neither the thesis nor substantial extracts from it may be printed or otherwise reproduced without his/her permission.
L'auteur a accordé une licence irrévocable et non exclusive permettant a la Bibliotheque nationale du Canada de reproduire, preter, distribuer ou vendre des coples de sa these de quelque maniere et sous quetque forme que ce soit pour mettre des exemplaires de cette thése à la disposition des personnes intéressées.

L'auteur conserve la propriété du droit d'auteur qui protège sa thèse. Ni la thése ni des extraits substantiels de celle-ci ne doivent etre imprimés ou autrement reproduits sans son autorisation.

$$
\text { I8BN } 0-315-58974-1
$$


The undersigned hereby recommend to the Faculty of Graduate Studies and Research acceptance of the thesis,

\section{ANALYSIS OF THE APPLICATION OF TRELLIS CODING TO SPREAD SPECTRUM MULTIPLE ACCESS SYSTEMS submitted by \\ GARY D. BOUDREAU}

in partial fulfillment of the requirements for the degree of Doctor of Philosophy

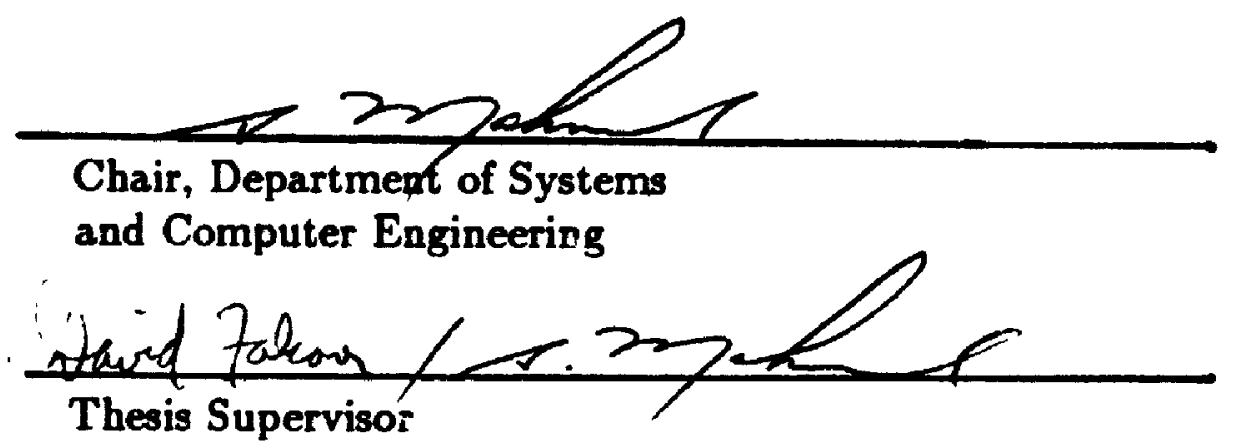
Tuperviso:

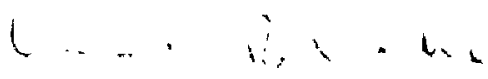

External Examiner

Carleton University 


\section{TO EMER AND CAITLIN}




\section{ABSTRACT}

The performance of trellis coding when it is applied to a spread spectrum multiple access (SSMA) system is examined. A system model is proposed that allows one to apply both trellis coding and a PN spreading sequence to the data symbols to be transmitted. Rate $\frac{n}{n+1}$, trellis codes employing $2^{n+1}$ point MPSK signal constellations are investigated when Gold sequences are employed for purposes of PN spreading. Performance in both an Additive White Gaussian Noise (AWGN) channel and a Rician fading channel is investigated, with 5-30 users transmitting simultaneously. Using criteria of equal complexity and throughput, the performance of the trellis codes in a SSMA environment is compared to that of medium to low rate convolutional codes through the use of a generalized transfer function bound in conjunction with the Chernoff bound. The average degradation due to the interuser interference is determined by emplcying the method of moments. The validity of approximating the interuser interference as a Gaussian random variable is also inrestigated. The numerical results illustrate that for a given complexity, chip rate and throughput, that low rate convolutional codes provide the best performance in a SSMA system. This is in spite of the fact that if lower rate convolutional codes are employed, there is an increase in the effective interuser interference due to the greater cross-corrclation effects from using shorter PN sequences, or alternatively from the effects of partial cross-correlation. However this increased degradation is more than overcome by the increased distance properties of the low rate codes. The coding gains for the codes that were considered range from 1-6dB (depending on the type and complexity of the code) over the corresponding uncoded performances of SSMA in an AllG.N channel. In the Rician fading channels the superiority of the convolutional codes over the trellis codes is more pronounced. 


\section{Acknowledgements}

I would like to express my appreciation for the help and guidance of Dr. David Falconer and Dr. Samy Mahmoud, without whom this work would not have been possible. I would also like to acknowledge the financial support of the Natural Sciences and Engineering Research Council in the form of NSERC Postgiaduate Scholarships, as well as the financial assistance provided by Canadian Astronatutirs Ltd., and the Telecommunications Research Institute of Ontario (TRIO). Finally I would like to acknowledge the support and patience of my wife Emer, throughout this work. 


\section{Contents}

Acceptance Sheet ii

Abstract iv

Acknowledgements v v

Table of Contents vi

List of Tables $\quad x$

List of Figures

$\begin{array}{ll}\text { Glossary of Notation } & \text { xvii }\end{array}$

Glossary of Abbreviatons $\quad$ xxiii

Chapter 1: Introduction 1

1.1 Literature Survey . . . . . . . . . . . . . . . . . 3

1.1.1 Spread Spertrum Multiple Access . . . . . . . . . . . 4

1.1.2 Code Sequence Analysis . . . . . . . . . . . . .

1.1 .3 Trellis Codirg . . . . . . . . . . . . . . 11

1.2 Thesis Organization . . . . . . . . . . . . 16

1.3 Thesis Contributions $\ldots \ldots \ldots \ldots \ldots$

$\begin{array}{lr}\text { Chapter 2: System Model } & 18\end{array}$

2.1 General system Architecture . . . . . . . . . . . . . 18 
2.1.1 Treilis Coded SSM.A . . . . . . . . . . . . . 20

2.1.2 Convolutionally Coded SSMA . . . . . . . . . . 26

2.1.3 Decoding ........................ 29

2.2 Derivation of Complex Interuser Interference $\ldots \ldots \ldots \ldots$

Chapter 3: Transfer Function Bounds 38

3.1 General Derivation . . . . . . . . . . . . . . . . 39

3.2 Transfer $F_{1}$ nction Bound Algorithm . . . . . . . . . . . . . 44

3.2.1 Four State 4-PSK Code $\ldots \ldots \ldots \ldots \ldots \ldots$

3.2.2 Four State 8-PSK Code $\ldots \ldots \ldots \ldots \ldots \ldots$

3.2.3 Encoder Diagrams . . . . . . . . . . . . . . 50

3.2.4 Convolutional Codes . . . . . . . . . . . . . . . .

3.3 Minimum Distance Computation $\ldots \ldots \ldots \ldots$

Chapter 4: Performance Analysis in an AWGN Channel 66

4.1 Exact Pairwise Error Probability . . . . . . . . . . . 677

4.2 Chernoff Bound Pairwise Error Probability . . . . . . . . . . . . 69

4.3 Chernoff Bound U'sing a Gaussian Approximation . . . . . . . it

Chapter 5: Performance Results in an AWGN Channel 75

5.1 Benchmark of Method of Moments . . . . . . . . . . if

5.2 Validity of the Independence Assumption . . . . . . . . is

5.3 Coded Q-function Bound . . . . . . . . . . . . . . . xu

5.4 Uncoded SSMA Bounds . . . . . . . . . . . . . . x(1)

5.5 Trellis Coded SSMA $\ldots \ldots \ldots \ldots \ldots \ldots \ldots$

$5.5 .1 \quad$ 4-PSK Codes $\ldots \ldots \ldots \ldots \ldots \ldots \ldots$

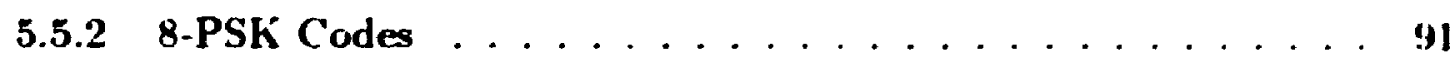

$5.5 .3 \quad 16-$ PSK Codes . . . . . . . . . . . . .

5.6 Convolutionally coded SSMA $\ldots \ldots \ldots \ldots$ (N)

5.6 .1 Rate $1 / 2$ Codes $\ldots \ldots \ldots \ldots \ldots$. . . . . . . . . . . . .

5.6 .2 Rate $1 / 8$ Codes . . . . . . . . . . . . . . 104

5.6 .3 Rate $2 / 3$ Codes . . . . . . . . . . . . . 104 
5.6.4 Rate $2 / 7$ Codes . . . . . . . . . . . . . . 111

5.7 Monte Carlo Simulations . . . . . . . . . . . . . . . . 114

5.8 Gaussian Approximation of the Interuser Interference . . . . . . 116

5.9 Partial Correlation Results . . . . . . . . . . . . . . 116

5.10 Summary . . . . . . . . . . . . . . . . . . 122

Chapter 6: Performance Analysis in a Fading Channel 126

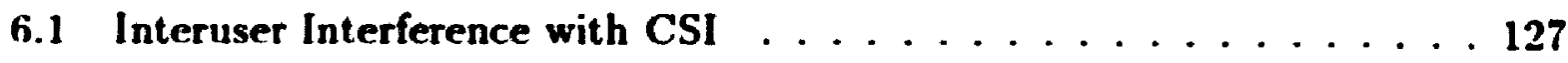

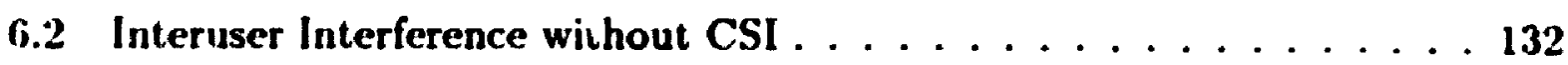

6.3 Fading channel with Gaussian assumption and CSI . . . . . . 135

6.4 Fading channel with Gaussian assumption and no CSI . . . . . . 138

Chapter 7: Performance Results in a Rician Fading Channel 141

i.1 Benchmark of Fading Bounds . . . . . . . . . . . . . 141

7.2 Trellis Codes with SSMA in Rician Fading . . . . . . . . . 143

7.3 Convolutional Codes with SSMA in Rician Fading . . . . . . . . 157

i.4 Gaussian Approximati in to IUI in Rician Fading ....... . 171

Chapter 8: Ccsolusion 182

8.1 Future Work. . . . . . . . . . . . . . . . . 184

Appendix A: Evaluation of the Moments of the Interuser Interference 186

A.1 Evaluation of Moments Inder the Independence Assumption . . . . 192

Appendix B: Moments of a Normalized Rician Random Variable 193

Appendix C: Exponential Integral 197

Appendix D: I and $Q$ Interuser Interference Analysis 199

D.1 Derivation of Interference . . . . . . . . . . . . . . 199

D.2 Evaluation of the Moments of the Interuser Interference . . . . . . 202

Appendix E: Correlation Moments of Common Constellations 206

E.1 :-i'Sh Constellation ................. . . . . . 
E.2 4-PSK Constellation $\ldots \ldots \ldots \ldots \ldots \ldots \ldots \ldots \ldots$

E.3 8-PSK Constellation . . . . . . . . . . . . . . 208

E.4 16-PSk Constellation . . . . . . . . . . . . . .208

Afpendix F: Moments of Product of Cosine and Sine Functions 213 Appendix G: Dependence of $z I_{,}$and $z Q$, 215 References 


\section{List of Tables}

2.1 Squared Euclidean distances between constellation signal points . . . 27

3.1 Superstate matrix for 4-state 4-PSK rate $1 / 2$ trellis code . . . . . 46

$3.28 \times 8$ superstate matrix for 4-state 4-PSK rate $1 / 2$ trellis code . . . 49

$3.34 \times 4$ superstate matrix for 4-state 4-PSK rate $1 / 2$ trellis code . . . 4 49

3.4 Superstate matrix for 4-state 8-PSK rate $2 / 3$ trellis code . . . . . 51

$3.58 \times 8$ superstate matrix for 4 -8tate 8-PSK rate $2 / 3$ trellis code . . . . 52

$3.64 \times 4$ superstate matrix for 4-state 8-PSK rate $2 / 3$ trellis code . . . 52

3.7 Generator polynomials of convolutional codes . . . . . . . . . 64

3.8 Comparison of the minimum distances of the trellis and convolutional codes studied . . . . . . . . . . . . . . . . 65

5.1 Performance of SSMA system with $K=2$ and $M$-sequences of length 31 it

5.2 Performance of SSMA system with $k=3$ and $M$-sequences of length 31 it

5.3 Comparison of trellis and convolutionally coded SSMA at $P_{e}=10^{-5}$

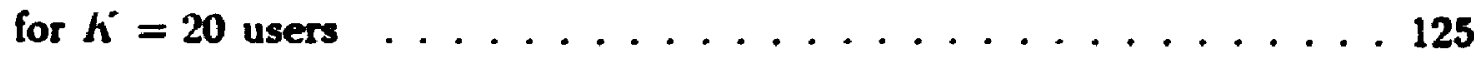

7.1 Comparison of trellis and convolut: $n$ ally coded SSMA at $P_{e}=10^{-5}$ for $K=20$ users in Rician fading channels . . . . . . . . 172 


\section{List of Figures}

2.1 System model. . . . . . . . . . . . . . . . 1!

2.2 Generalized transmitter basebas.t system model. . . . . . . 21

2.3 Partitioning of 8-PSK signal constellation. . . . . . . . . 22

2.4 Antipodal cosets and Euclidean distances of 4-PSK $\ldots \ldots \ldots .23$

2.5 Antipodal cosets and Euclidean distances of 8-PSK. . . . . . . 24

2.6 Antipodal cosets and Euclidean distances of 16-PSK. . . . . . 25

2.7 Trellis state diagram of rate $1 / 2$ 4-state 4-PSK trellis code $\ldots \ldots .28$

2.8 Receiver model $\ldots \ldots \ldots \ldots \ldots \ldots \ldots \ldots \ldots \ldots$

3.1 Systematic 2-state rate 1/2 4-PSK and 2-state rate 2/3 8-PSK trellis encoders ...........................

3.2 Systematic 4-state rate $1 / 2$ 4-PSK, 4-state rate $2 / 3$ 8-PSK and 4 state rate $3 / 4$ 16-PSK trellis encoders . . . . . . . . . . . 5

3.3 Systematic 8 -st 2 te rate $1 / 2$ 4-PSK trellis encoder $\ldots \ldots \ldots \ldots$

3.4 Systematic 8-state rate 2/3 8-PSK and 8-state rate 3/4 16-PSK trellis

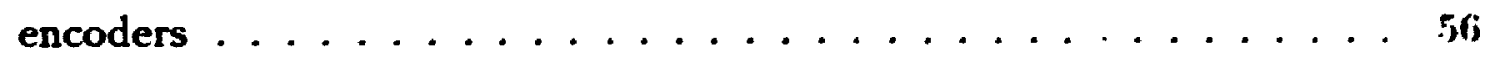

3.5 Systematic 16 -state rate $2 / 3$ 8-PSK trellis encoder . . . . . . . . i7

3.6 Encoder for the 16-PSK 16-state rate $3 / 1$ trellis code . . . . . . . i,

3.7 Encoder for the 4 -state rate $1 / 2$ convolutional code $\ldots \ldots \ldots$. . . (i)

3.8 Encoder for the 4 -state rate $2 / 7$ convolutional code . . . . . . . . (i)

5.1 A comparison of the Chernoff bound for dependent versus independent moments for path lengths of 2 and $3 \ldots \ldots \ldots$. . . . . . . 
5.2 Performance of Q-function bound versus Chernoff bound for the 4 state 4-PSK trellis code. . . . . . . . . . . . . . 81

5.3 Comparsion of Q-function : ound versus Chernoff bound for 4 and 8 state rate $1 / 2$ convolutional codes $\ldots \ldots \ldots \ldots \ldots$. . . . 82

5.4 Performance of uncoded SSMA for $K=10$ users . . . . . . . . 84

5.5 Performance of uncoded SSMA for $K=20$ users . . . . . . . . 8 85

5.6 Chernoff parameter $\lambda$ as a function of $\frac{E_{t}}{N_{0}}$ for a branch of distance 2. . 87

5.7 Performance of 4-PSK 2-state trellis codes for $K=10$ and $K=20$ users

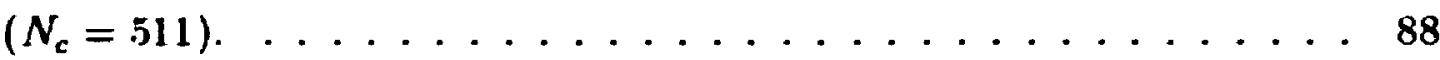

5.8 Performance of 4-PSK 4-state trellis codes for $K=10$ and $K=20$ users $\left(N_{c}=\$ 11\right)$.

5.9 Performance of 4 -PSK 8-state trellis codes for $K=10$ and $K=20$ users $\left(N_{c}=511\right)$.

5.10 Performance of 8-PSK 2-state trellis codes for $K=10$ and $K=20$ users $\left(N_{c}=511\right)$

5.11 Performance of 8-PSK 4-state trellis codes for $K=10$ and $K=20$ users $\left(N_{c}=511\right)$.

5.12 Performance of 8-PSK 8-state trellis codes for $K=10$ and $K=20$ users $\left(N_{c}=511\right)$

5.13 Performance of 8-PSK 16 -state trellis codes for $K=10$ and $K=20$ users $\left(N_{i}=511\right)$.

5.14 Performance of 16-PSK 4-state trellis codes for $K=10$ and $K=20$ users $\left(N_{c}=511\right)$.

5.15 Performance of 16-PSK 8-state trellis codes for $\mathrm{K}=10$ and $\mathrm{K}=20$ users $\left(N_{\mathrm{c}}=511\right)$.

5.16 Performance of 16-PSK 16-state trellis codes for $K=10$ and $K=20$ users $\left(N_{c}=511\right)$

5.17 Performance of rate $1 / 24$-state convolutional codes for $K=10, K=20$ and $k=30$ users $\left(N_{c}=255\right) \ldots \ldots \ldots \ldots \ldots 1 \ldots \ldots \ldots \ldots$

5.18 Performance of rate $1 / 2$ 8-state convolutional codes for $\mathrm{K}=10$, and $h=20$ users $\left(N_{r}=255\right)$. 
5.19 Performance of rate $1 / 216$-state convolutional codes for $k=10$, and

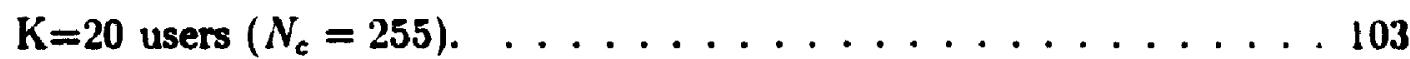

5.20 Performance of raie $1 / 8$ 4-state convolutional codes for $k=10$ and $\mathrm{K}=20$ users $\left(N_{c}=63\right)$.

5.21 Performance of rate $1 / 8$ 8-state convolutional codes for $k=10$ and

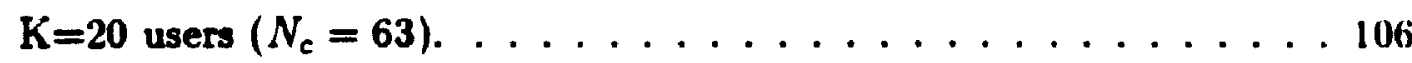

5.22 Performance of rate $1 / 8 \quad 16$-state convolutional codes for $\mathrm{h}=10$ and $\mathrm{K}=20$ users $\left(N_{c}=63\right)$.

5.23 Performance of rate $2 / 3$ 4-state convolutional codes for $k=10$ and $\mathrm{K}=20$ users $\left(N_{\mathrm{c}}=127\right)$.

5.24 Performance of rate 2/3 16 -state convolutional codes for $K=10$ and $\mathrm{K}=20$ users $\left(N_{c}=127\right)$.

5.25 Performance of rate $2 / 7$ 4-state convolutional codes for $k=10$ and $\mathrm{K}=20$ users $\left(N_{c}=63\right)$.

5.26 Performance of rate $2 / 7$ 16-state convolutional codes for $h=10$ and $\mathrm{K}=20$ users $\left(N_{c}=63\right)$.

5.27 Performance of Monte Carlo simulation for rate 4-state rate 1/2 4PSK trellis code versus 4 -state rate $1 / 2$ convolutional code

5.28 Performance of 4-PSK 4-state trellis code for $K=10$ and $K=20$ users employing the Gaussian Approximation $\left(N_{c}=511\right) \ldots \ldots 117$

5.29 Performance of 8-PSK 4-state trellis code for $k=10$ and $k=20$ isers employing the Gaussian Approximation $\left(N_{c}=511\right) \ldots \ldots \ldots$. . . .

5.30 Performance of 8-PSK 16-state trellis code for $K=10$ and $K=20$ users employing the Gaussian Approximation $\left(N_{c}=511\right) \ldots \ldots \ldots$ 119

5.31 Performance of rate $1 / 8$ 4-state convolutional code with $K=10$ and $\mathrm{K}=20$ users employing the Gaussian Approximation $\left(N_{c}=63\right) \ldots 120$

5.32 Performance of rate $2 / 7$ 4-state convolutional code with $K=10$ and $\mathrm{K}=20$ users employing the Gaussian Approximation $\left(N_{c}=63\right) . \ldots 121$

5.33 Performance of rate $1 / 7$ 4-state convolutional code for $\mathrm{K}=10$ users $\left(N_{c}=511\right)$. Each coded symbol spans a subsequence of 73 chips . . . 123 
7.1 Performance of the 2-state 4-PSK trellis code in Rician fading without

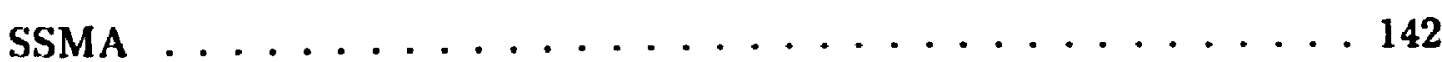

7.2 Performance of the 4-state 4-PSK trellis code in Rician fading without SSMA . . . . . . . . . . . . . . . . 144

7.3 Performance of 4-state rate $1 / 8$ convolutional code in Rician fading without SSMA . . . . . . . . . . . . . 145

7.4 Performance of uncoded BPSK in Rician fading with CSI . . . . . 146

7.5 Performance of uncoded BPSK in Rician fading with

7.6 Performance of uncoded 4-PSK in Rician fading with CSI . . . . . 148

7.7 Performance of uncoded 4-PSK in Rician fading without CSI . . . 149

7.9 Performance of 4-PSK 4-state trellis code for $\mathrm{K}=20$ users in a Rician fading channel with $\left(\right.$ SI for $K_{f}=10.0,1.0$ and $0.0\left(N_{c}=511\right) \ldots 151$

7.9 Performance of 4-PSK 4-state trellis code for $\mathrm{K}=20$ users in a Rician fading channel with no CSI for $K_{f}=10.0,1.0$ and $0.0\left(N_{c}=511\right) \ldots 152$

7.10 Performance of 8-PSK 4-state trellis code for $\mathrm{K}=\mathbf{2 0}$ users in a Rician fading channel with CSI for $K_{f}=10.0,1.0$ and $0.0\left(N_{c}=511\right) . \ldots 153$

T.11 Performance of 8-PSK 4-state trellis code for $\mathrm{K}=\mathbf{2 0}$ users in a Rician fading channel with no CSI for $K_{j}^{\prime}=10.0,1.0$ and $0.0\left(N_{c}=511\right) \ldots 154$

7.12 Performance of 8-PSK 16-state trellis code for $\mathrm{K}=20$ users in a Rician fading channel with CSI for $K_{f}=10.0$ and $1.0\left(N_{c}=511\right) . \ldots 155$

7.13 Performance of 8-PSK 16-state trellis code for $\mathrm{K}=20$ users in Rician fading with no CSI for $K_{f}=10.0,1.0$ and $0.0\left(N_{c}=511\right) \ldots \ldots 156$

T.14 Performance of rate 1/2 4-state convolutional code for $\mathrm{K}=20$ users in Rician fading with CSI for $K_{f}=10.0,1.0$ and $0.0\left(N_{c}=255\right) . \ldots 158$

7.15 Performance of rate $1 / 2$ 4-state convolutional code for $K=20$ users in Rician fading without CSI for $K_{f}=10.0,1.0$ and $0.0\left(N_{c}=255\right) \ldots 159$

7.16 Performance of rate 1/8 4-state convolutional code for $K=20$ users in Rician fading with CSI for $K_{j}=10.0,1.0$ and $0.0\left(N_{c}=63\right) \ldots \ldots 160$

7.17 Performance of rate 1/8 4-state convolutional code for $K=20$ users in Rician fading without CSI for $K_{f}=10.0,1.0$ and $0.0\left(N_{c}=63\right) . \ldots 161$ 
7.18 Performance of rate $1 / 216$-state convolutional code for $h=20$ users in Rician fading with CSI for $K_{f}=10.0 .1 .0$ and $0.0\left(N_{c}=255\right) \ldots$ 162

7.19 Performance of rate $1 / 2$ 16-state convolutional code for $K=20$ users in Rician fading without CSI for $K_{f}=10.0,1.0$ and $0.0\left(N_{r}=255\right) . \quad .163$

7.20 Performance of rate $1 / 8$ 16-state convolutional code for $h=20$ users in Rician fading with CSI for $K_{f}=10.0,1.0$ and $0.0\left(N_{c}=63\right) \ldots \ldots 164$

7.21 Performance of rate $1 / 8$ 16-state convolutional code for $k=20$ users in Rician fading without CSI for $K_{f}=10.0,1.0$ and $0.0\left(N_{c}=63\right)$. . 165

7.22 Performance of rate $2 / 7$ 4-state convolutional code for $K=20$ users in Rician fading with CSI for $K_{f}=10.0,1.0$ and $0.0\left(N_{c}=63\right) \ldots \ldots$ I66

7.23 Performance of rate $2 / 7$ 4-state convolutional code for $h=20$ users in Rician fading without CSI for $K_{f}=10.0,1.0$ and $0.0\left(N_{c}=63\right) . \ldots 16 i$

7.24 Performance of rate $2 / 7$ 16-state convolutional code for $K=20$ users in Rician fading with CSI for $K_{f}=10.0,1.0$ and $0.0\left(N_{c}=63\right) \ldots \ldots 168$

7.25 Performance of rate $2 / 7 \quad 16$-state convolutional code for $k=20$ users in Rician fading without CSI for $K_{f}=10.0,1.0$ and $0.0\left(N_{c}=63\right) \ldots 169$

7.26 Performance of 4-PSK 4-state trellis code in Rician fading with CSI for $K=20$ users employ ing the Gaussian Approximation $\left(N_{c}=511\right) . \quad 174$

7.27 Performance of 4-state rate $1 / 8$ convolutional code in Rician fading with CSI for $\mathrm{K}=20$ users comploying the Gaussian Approximation $\left(N_{c}=63\right)$

7.28 Performance of 8-PSK 16-state trellis code in Rician fading with ('S) for $\mathrm{K}=20$ users employing the Gaussian Approximation $\left(N_{c}=511\right)$. . 176

7.29 Performance of 16 -state rate $2 / 7$ convolutional code in Rician fading with $\mathrm{CSI}$ for $\mathrm{K}=20$ users employing the Gaussian Approximation

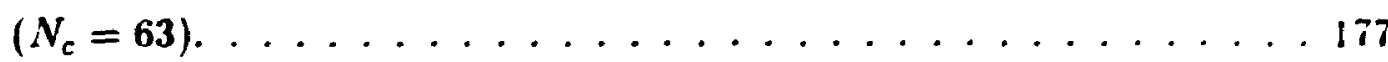

7.30 Performance of 4-PSK 4-state trellis code in Rician Fading without CSI for $K=20$ users employing the Gaussian Approximation $\left(N_{r}=.111\right) .17 \mathrm{~K}$

7.31 Performance of 8-PSK trellis codes in Rician fading without (' $\mathrm{SI}$ for $K=20$ users employing the Gaussian Approximation $\left(N_{c}=511\right)$. 
7.32 Performance of 16-state rate $1 / 8$ convolutional code in Rician fading without CSI for $K=20$ users employing the Gaussian Approximation

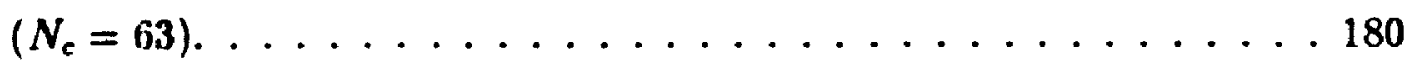

7.33 Performance of 16-state rate $2 / 7$ convolutional code in Rician fading without CSI for $\mathrm{K}=20$ users employing the Gaussian Approximation

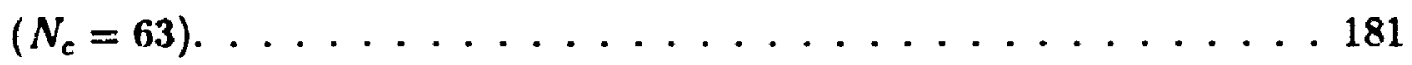

E.1 Signal constellation of 4-PSK with labelled I and $Q$ components . . 210

E.2 8-PSK signal constellation with labelled $I$ and $Q$ components. . . . 211

E.3 16-PSK signal constellation with labelled $I$ and $Q$ components. . . . 212 


\section{GLOSSARY OF NOTATION}

\begin{tabular}{|c|c|}
\hline $\mathbf{a}_{(r)}^{i}$ & $-r^{\text {th }}$ subsequence of $a^{i}(t)$ \\
\hline$a^{k}(t)$ & - direct sequence spreading code signal of the $k^{\text {th }}$ user \\
\hline$a_{m}^{k}$ & - $m^{\text {th }}$ chip of the PN sequence of the $k^{\text {th }}$ user \\
\hline$a(I)$ & - probability of a transition between 'good' super states \\
\hline $\mathbf{b}(I)$ & $\begin{array}{l}\text { - vector containing transition probabilities from good to bad } \\
\text { super states }\end{array}$ \\
\hline$b^{k}(t)$ & - real information signal of the $k^{\text {th }}$ user \\
\hline$b_{p}^{k}$ & - the amplitude of $x_{p}^{k}$ \\
\hline$C[\mathbf{a}, \mathbf{b}](l)$ & $\begin{array}{l}\text { - aperiodic cross-correlation function between subsequences } \\
\mathbf{a} \text { and } \mathbf{b}\end{array}$ \\
\hline$C_{k, i}(l)$ & $\begin{array}{l}\text { - the aferiodic cross-correlation function between sequences } k \text { and } \\
i \text { at a relative phase offset of } l\end{array}$ \\
\hline $\mathbf{c}(I)$ & $\begin{array}{l}\text { - vector containing transition probabilities from bad to good } \\
\text { super states }\end{array}$ \\
\hline$C\left(\alpha_{i}\right)$ & - Chernoff bound for Euclidean distance $\alpha_{i}$ \\
\hline $\mathbf{D}$ & - error pattern vector \\
\hline$D_{l_{p}}$ & - in-phase component of $\mathbf{D}$ at time $p$ \\
\hline$D_{Q_{p}}$ & - quadrature component of $\mathbf{D}$ at time $p$ \\
\hline$D_{\lambda}(\mathbf{S}, \mathbf{U})$ & - Bhattacharyya bound between $\mathbf{S}$ and $\mathbf{U}$ \\
\hline d & - energy normalized error pattern vector \\
\hline$d_{I_{p}}$ & - in-phase component of $d$ at time $p$ \\
\hline$d_{Q_{p}}$ & - quadrature component of $d$ at time $p$ \\
\hline$d(\mathbf{U})$ & - Hamming weight of $\mathbf{U}$ \\
\hline$d\left(U_{p}\right)$ & - Hamming weight of $U_{p}$ \\
\hline $\mathbf{E}[\cdot]$ & - expected value symbol \\
\hline$E_{p}$ & - the real (in-phase) part of $G_{p}$ \\
\hline$E$. & - symbol energy \\
\hline $\mathbf{e}$ & - the base of the natural logarithm (i.e. $2.71 \times 2 \times 1 \ldots$ ) \\
\hline
\end{tabular}




\begin{tabular}{|c|c|}
\hline$F_{p}$ & - the imaginary (quadrature) part of $G_{p}$ \\
\hline$f(\cdot)$ & - a nonlinear function of $u_{p}$ and $\delta_{p}$ \\
\hline$f_{c}$ & - carrier frequency \\
\hline$G_{d}$ & - the greatest common divisor of $\mathrm{L}$ and $N_{c}$ \\
\hline$G_{p}^{\prime}$ & $\begin{array}{l}\text { - amplitude and phase normalized interuser interference seen } \\
\text { by the } i^{\text {th }} \text { user in the } p^{\text {th }} \text { time interval }\end{array}$ \\
\hline$g(\cdot)$ & - a nonlinear function of $u_{p}$ and $\delta_{p}$ \\
\hline $\mathbf{I}$ & - the identity matrix \\
\hline 1 & $\begin{array}{l}\text { - indeterminate variable employed to tag bit errors in the transfer } \\
\text { function bound }\end{array}$ \\
\hline$l_{0}(\cdot)$ & - zeroth order Bessel function of the first kind \\
\hline j & $\cdot \sqrt{-1}$ \\
\hline $\mathbf{K}$ & - number of SSMA users \\
\hline $\boldsymbol{K}_{\mathrm{s}}$ & - the Rician fading parameter \\
\hline k & - the number of input bits to the trellis or convolutional coder in a baud \\
\hline$k_{1}$ & - the number of coded input bits in a trellis code \\
\hline$k_{2}$ & - the number of uncoded input bits in a trellis code \\
\hline L & - the length of a subsequence of a PN sequence \\
\hline $\mathbf{M}$ & - encoding constraint length of the trellis or convolutional code \\
\hline $\mathbf{m}$ & - number of states in a code \\
\hline$m(\mathbf{X} . \mathbf{Y})$ & - the maximum likelihood metric between $\mathbf{X}$ and $\mathbf{Y}$ \\
\hline$N_{c}$ & - the number of chips in a PN sequence \\
\hline$N_{m}$ & - the order up to which moments are calculated \\
\hline$N_{0}$ & - one sided spectral density of $A W G N$ \\
\hline$n(t)$ & - AWGN process with variance $N_{0} / 2$ \\
\hline $\mathbf{n}$ & - the number of coded symbols in a convolutional code \\
\hline$P_{b}$ & - the probability of bit error \\
\hline$P_{E}$ & - the probability of a pairwise error event \\
\hline$P\left(S_{\mathbf{0}}\right)$ & - the probability of being in super state $S_{0}$ \\
\hline $\operatorname{Pr}(\cdot)$ & - rectangular pulse of $\mathrm{T}$ seconds duration \\
\hline
\end{tabular}




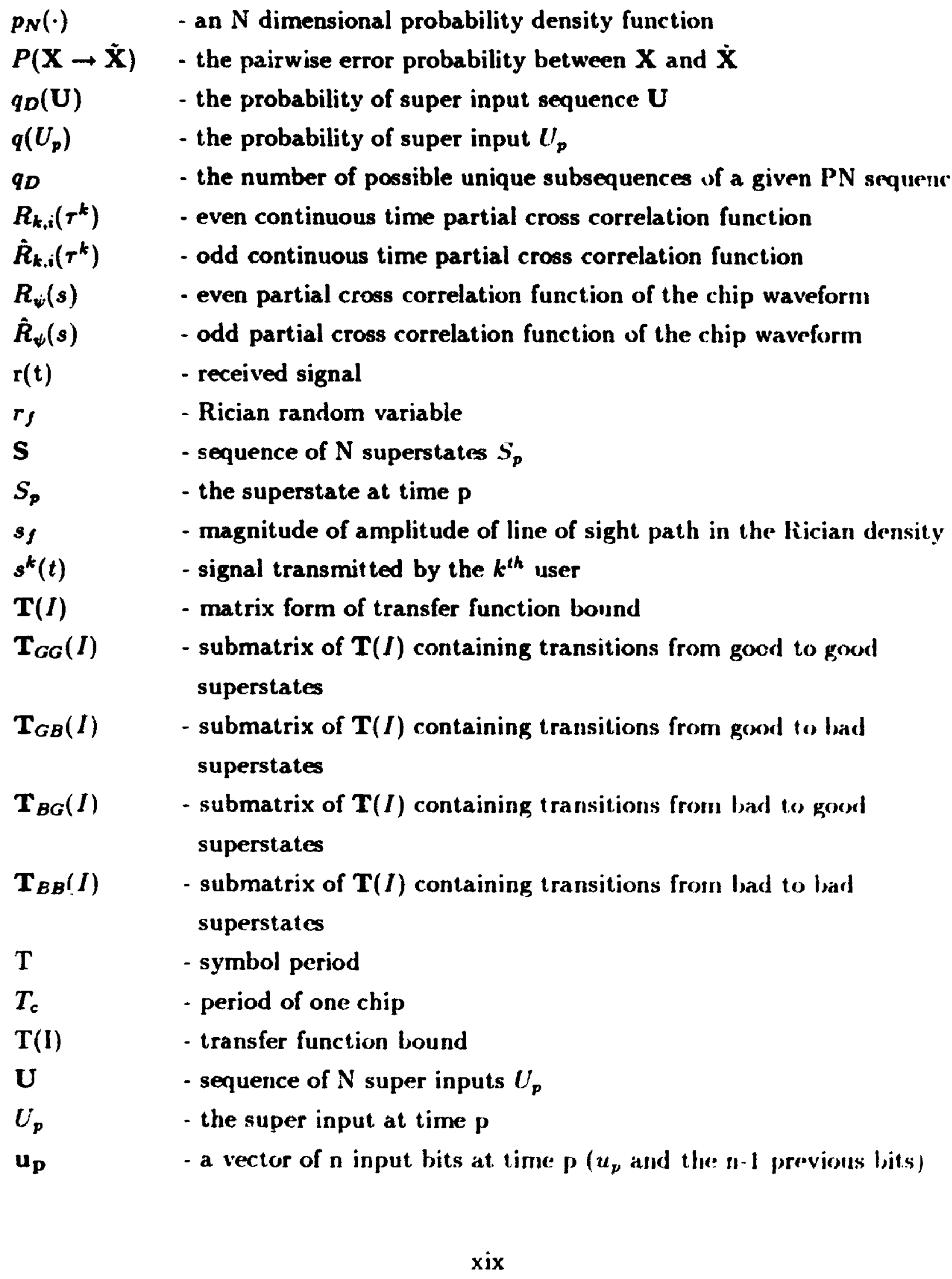




\begin{tabular}{|c|c|}
\hline$\tilde{\mathbf{u}}_{p}$ & - a vector of $n$ input bits at time $p$ for the error sequence \\
\hline$u_{p}$ & - the input bit at time $p$ \\
\hline $\mathbf{V}$ & - joint IUI random vector \\
\hline $\mathbf{v}$ & - energy normalized joint IUI random varialle \\
\hline$W_{\mathrm{p}}$ & $\begin{array}{l}\text { - the } i^{\text {th }} \text { weight from the method of moments for the } p^{\text {th }} \text { time } \\
\text { interval }\end{array}$ \\
\hline $\mathbf{X}$ & - complex baseband channel input sequence \\
\hline$\dot{\mathbf{x}}$ & - complex baseband channel error event input sequence \\
\hline $\boldsymbol{X}_{p}$ & - complex baseband symbol transmitted in the $p^{\text {th }}$ time interval \\
\hline$x^{k}(t)$ & - normalized complex baseband information signa! of the $k^{\text {th }}$ user \\
\hline $\boldsymbol{r}_{p}^{k}$ & $\begin{array}{l}\text { - normalized complex baseband information symbol of the } k^{\text {th }} \text { user } \\
\text { at time } \mathrm{p}\end{array}$ \\
\hline$r I_{p}$ & - in-phase (real) component of $x_{p}^{k}$ \\
\hline$x_{Q_{p}}$ & - quadrature (imaginary) component of $x_{p}^{k}$ \\
\hline $\mathbf{Y}$ & - romplex baseband ahannel uutput symbol sequence \\
\hline$Y(t)$ & - equivalent complex lowpass output of the correlation process \\
\hline$Y_{p}^{\prime}$ & $\begin{array}{l}\text { - equivalent complex lowpass output symbol of the correlation } \\
\text { process of the } i^{\text {th }} \text { user at time } p\end{array}$ \\
\hline $\mathbf{Z}$ & - complex baseband sequence of interuser interference \\
\hline$Z_{n}^{2}$ & $\begin{array}{l}\text { - a sample of interuser interference seen by the } i^{\text {th }} \text { user in the } \\
p^{\text {th }} \text { time interval }\end{array}$ \\
\hline$z_{p}^{\prime}$ & $\begin{array}{l}\text { - energy normalized sample of the IUI seen by the } i^{\text {th }} \text { user in } \\
\text { the } p^{\text {th }} \text { time interval }\end{array}$ \\
\hline$=I_{p}$ & - in-phase (real) component of $z_{p}^{k}$ \\
\hline$\approx Q_{p}$ & - quadrature (imaginary) component of $z_{p}^{k}$ \\
\hline 1 & the all ones column vector \\
\hline $\begin{array}{l}\alpha_{1} \\
s^{k}\end{array}$ & $\begin{array}{l}\text { - Euclidean distance between two signal points } x_{i} \text { and } \dot{x}_{i} \\
\text { - random phase of the carr:ar of the } k^{\text {th }} \text { user at the receiver }\end{array}$ \\
\hline$\delta_{p}$ & - the state of the encoder at time $p$ \\
\hline$i_{p}$ & - the incorrect state of the encoder at time $p$ \\
\hline
\end{tabular}




\begin{tabular}{|c|c|}
\hline$\mu_{c}$ & - angular carrier frequency \\
\hline$\phi^{k}$ & - random phase of the carrier of the $k^{\text {th }}$ user at the transmitter \\
\hline$\Phi i(t)$ & - in phase orthonormal carrier of the $i^{\text {th }}$ user \\
\hline$\Phi_{2}^{i}(t)$ & - quadrature orthonormal carrier of the $i^{\text {th }}$ user \\
\hline$\eta_{p}^{i}$ & $\begin{array}{l}\text { sample of complex AWGN at the output of the correlation } \\
\text { process with variance } N_{0}\end{array}$ \\
\hline$\eta I$ & - the real part of $\eta_{p}^{2}$ \\
\hline$\eta_{Q}$ & - the imaginary part of $\eta_{p}^{i}$ \\
\hline$\theta_{p}^{k}$ & - the phase of $x_{p}^{\kappa}$ \\
\hline$\lambda$ & - Chernoff bound parameter \\
\hline$\nu$ & - set of all $p$ such that $X_{p} \neq \dot{X}_{p}$ \\
\hline$\sigma_{z}^{2}$ & - the variance of the IUI random variable \\
\hline$\zeta_{p_{\text {s }}}$ & - the $i^{\text {th }}$ node from wae method of moments in the $p^{\text {th }}$ time interval \\
\hline$\rho_{p}$ & $\begin{array}{l}\text { - the normalized faded amplitude on the transmittod symboi during } \\
\text { the } p^{\text {th }} \text { time interval }\end{array}$ \\
\hline$\rho^{k}$ & - the normalized faded amplitude on the IUI from the $h^{\text {th }}$ user \\
\hline$\Lambda$ & - a lattice \\
\hline$\Lambda^{\prime}$ & - a sublattice of $\Lambda$ \\
\hline$\tau^{k}$ & - random delay of the $k^{\text {th }}$ user \\
\hline $4(\cdot)$ & - chip waveform \\
\hline$\xi^{k}(t)$ & - complex baseband direct sequence spread signal of the $k^{\text {th }}$ user \\
\hline $\mathcal{Z}\left(z_{p}, z_{p}^{*}\right)$ & - intermediate product of $z_{p}$ and $z_{p}^{*}$ used in the moment calculations \\
\hline $\mathcal{Z}^{\prime}\left(z_{l}, z_{Q}\right)$ & - intermediate product of $z$, and $z_{Q}^{*}$ used in the momknt calculations \\
\hline $\mathcal{G}\left(G_{p}, G_{p}^{*}\right)$ & $\begin{array}{l}\text { - intermediate product of } g_{p} \text { and } r_{p}^{*} \text { userl in the monnent } \\
\text { calculations }\end{array}$ \\
\hline $\mathcal{F}\left(E_{p}, F_{p}\right)$ & $\begin{array}{l}\text { - intermediate product of } E_{p} \text { and } F_{p} \text { used in the mosnent } \\
\text { calculations }\end{array}$ \\
\hline $\mathcal{X}\left(x_{I_{p}}^{k} \cdot x_{Q_{p}}^{k}\right)$ & $\begin{array}{l}\text { - intermediate product of } x_{I_{p}}^{k} \text { and } x_{Q_{p}}^{k} \text { used in the moment } \\
\text { calculations }\end{array}$ \\
\hline $\mathcal{R}(R, \hat{R})$ & - intermediate product of $R_{k, x}\left(\tau^{k}\right)$ and $\dot{R}_{k, 1}\left(\tau^{k}\right)$ itsed in the moment \\
\hline
\end{tabular}


calculations

xxii 


\section{GLOSSARY OF ABBREVIATIONS}

$\begin{array}{ll}\text { AWGN } & \text { - Additive White Gauseian Noise } \\ \text { BCH } & \text { - Bose-Chaudhuri-Hocquenghem } \\ \text { BPSK } & \text { - Binary Phase Shift Keying } \\ \text { CSI } & \text { - Channel State Information } \\ \text { dB } & \text { - decibel } \\ \text { DPSK } & \text { - Differential Phase Shift Keying } \\ \text { DS } & \text { - Direct Sequence } \\ \text { I } & \text { - in-phase } \\ \text { ISI } & \text { - Intersymbol Interference } \\ \text { IUI } & \text { - Interuser Interference } \\ \text { MHz } & \text { - MegaHertz } \\ \text { MPC } & \text { - Multiphase Complementary Codes } \\ \text { MPSK } & \text { - M-ary Phase Shift Keying } \\ \text { MSK } & \text { - Minimum Phase Shift Keying } \\ \text { MTCM } & \text { - Multiple Trellis Coded Modulation } \\ \text { OQPSK } & \text { - Offset Quadrature Phase Shift Keying } \\ \text { PN } & \text { - Pseudonoise } \\ \text { Q } & \text { - quadrature } \\ \text { QAM } & \text { - Quadrature Amplitude Modulation } \\ \text { QPSK } & \text { - Quadrature Phase Shift Keying } \\ \text { SAW } & \text { - Surface Acoustic Wave } \\ \text { SS } & \text { - Spread Spectrum } \\ \text { SSMA } & \text { - Spread Spectrum Multiple Access } \\ \text { TCM } & \text { - Trellis Coded Modulation } \\ \text { TDMA } & \text { - Time Division Multiple Access } \\ \end{array}$




\section{Chapter 1}

\section{Introduction}

There has been recent interest in the use of direct sequence (DS) spread spectrum inultiple arcess (SSMI ) for use in an indoor wireless local area network, in the 800 MIlz to $1200 \mathrm{MIIz}$ frequency range. Kavehrad in conjunction with other authors $[1-5]$ has investigated the use of BPSK and DPSK in conjunction with DS spreading as an alternative communication technique to TDMA in an indoor wireless radio environment.

The pluses of DS spread spectrum over a TDMA architecture include [1]: (i) multiple acce.. is asynchronous as opposed to the relatively complex synchronization circuitry required in a TDMA system: (ii) inherent diversity to multipath: (iii) frequency re-use is possible if a star local area network is employed; (iv) spread spectrum is robust to time varying channel conditions and finally (v) SS provides built in addressing and security.

Disad vantages of employing a DS spread spectrum system as opposed to a TDMA scheme. include: (i) the near/far problem: (ii) interference to existing systems (i.e. if the spread spectrum system is overlaid on frequency bands currently being used), and (iii) the inferior probability of error performance possible for a given number of users.

Kawhrad has analyzed DS spread spectrum (using BPSK and DPSK) for various 
types of diversity and coding [1][2\};3] in an indoor wireless Rayleigh fading multipath environment. This analysis emp oyed Gold and Kasami codes for DS spreading of the transmitted waveforms. The results of these analyses conclude that traditional DS SSMA schemes provide poor performance in a Rayleigh fading environment unless some form of diversity is provided. It should be noted that the above studies made no attempt to optimize the DS spreading code.

Rece.at research into trellis codes has demonstrated that coding gains of 3-6dB are possible with no bandwidth expansion, making them ideally suited to bandlimited channels[6][7]. It is proposed that the use of trellis codes in a DS-SSMA system be investigated to ascertain if similar improvements in the performance of a SSMA system can be obtained. In the system to be studied, the data sequence is trellis coded before being spread by a pseudo-random sequence. Such an approach allows one to obtain coding gain without any resulting reduction in throughput. For purposes of comparison, the performance of convolutionally coded SSMA systems are also investigated. The comparison is performed on a basis of equal throughput and complexity. In order to maintain the same throughput as an uncoderd system, the convolutionally coded system must either use shorter PN sequences or pay a penalty due to additional interuser interference degradation from partial cross-correlation effects. The magnitude of the degradation due to partial cross-correlation will be examined in relation to increased coding gain due to the greater minimum distance of the lower rate convolutional codes.

Since any actual implementation of a SSMA system is likely to encounter fading (i.e. such as in indoor wireless radio channels [2][8][9], or cellular telephone rhannels), a the ough comparison of the performance of trellis coded SSMA to convelutionally coded : " TA must also include an analysis of a fading environment. As such, using the same comparison criteria as for the AWGN channel. both types of coding will also be investigated for a SSMA channel operating in a Rician fading chanme- Various ratios of the direct line of sight power to diffuse power will be examinerl. Althenght 
the Rician model of a fading channel may not be a completely realistic replica of the types of degradations to be encountered in a fading environment, it does illustrate the i,ypes of effects and performance that can be expected in a fading channel.

In addition to the channel impairments of AWGN or fading, as the case may be, the principal problem of evaluating the performance of a SSMA system is to adequately model the degradation due to interuser interference. It is possible to model this impairment as Gaussian noise, however such an assumption is in general not always 'iate[26]. Pursley et al.[12] [19] have developed notation and analysis to account for the interuser interference in an exact manner. Once having accurately mathematically modelled the interuser interference, the crux of the problem is to estimate the probability distribution function or equivalently the probability density function of the interuser interference, since in general the density function is unknown. Using Pursley's analysis it is possible to calculate the moments of this unknown density. from which there are a variety of techniques available to estimate the density function accurately (i.e. such as the method of moments[23] or the maximum entropy technique [24]). The method of moments was employed in this study to evaluate the interuser interference, in conjunction with standard transfer function bound techniques[90] to bound the average performance of the trellis or convolut ional codes.

\section{$1.1 \quad$ Literature Survey}

This work brings together theory from three areas of research in communication theory; namely. (i) indoor wireless radio and SSMA performance evaluation: (ii) psendorandom code sequence analysis and finally (iii) trellis coding. The following werview of the currently available relevant papers in the open literature is divided into these sections. 


\subsubsection{Spread Spectrum Multiple Access}

This section outlines the significant recent studies of asynchronous SSMA systems. As described in the introduction, Kavehrad, in conjunction with other authors has examined the performance of SSMA with and without coding and/or diversity, in an indoor wireless fading channel [1][2][3][4][5]. The fading multipath model employed in the analysis is based on two common channel models, namely, the models of Turin[10] and Saleh and Valenzuela [11]. Turin's propagation model characterizes the impulse response as a discrete time summation of $K$ paths, each with a random phase $\theta_{k}$, a random time delay $t_{k}$ and a random path gain $a_{k}$. The second channes model of Saleh and Valenzuela[11] is similar to Turin's model, with the difference that the discrete paths were assumed to arrive in clusters with the arrival times of the clusters forming a Poisson process of some fixed rate $\Lambda$. Furthermore within each cluster subsequent rays arrive according to a second Poisson process of fixed rate $\lambda$.

Kavehrad also examined var.ous diversity schemes such as selection diversity, equal gain combining and maximal ratio combining (MRC) when more than one antenna is present per receiver, whereas predetection combining was employed to analyze a spread spectrum (SS) system using DPSK modulation. The results of the analysis of various receiver structures demonstrated that diversity provided significant gains in performance, with maxirnal ratio combining being superior to selection diversity with bluck coding.

In order to ameliorate the performance of SSMA in a Rayleigh: fading multipatl, environment, Kavehrad and McLane[3] investigated the use of selection diversity and the use of a $(7,4)$ Hamming code as well as a $(15.7) \mathrm{BCH}$ code. The combination of selection diversity and error correction coding significant!y reduced the probability of the error rate noise floor when compared to the $n$ indiversity case previously described. The validity of the Gaussian assumption was also investigated for the: system under consideration and it was found to be in error by up to $20 \%$.

The above studies involved use of coherent detertion for biphase modulated DS. 
SSMA. Kavehrad and Ramamurthi[4] investigated the performance of an indoor wireless communications SSMA system employing DPSK modulation and diversity. A SAW filter was assumed to be employed as a matched filter (matched to the user's PN sequence) and diversity was achieved either throug.1 the use of predetection combining or selection diversity.

In order to evaluate the system performance of the predetection combining diversity DPSK receiver Kavehrad had to resort to the Gaussian assumption and use a Monte Carlo type simulation. It was found that the noise floor for predetection combining was an order of magnitude superior (in terms of $\boldsymbol{P}_{e}$ ) over that of selection diversity. This difference in performance became more pronounced as the order of diversity increased. The performance with predetection combining was also shown to compare favourably with the demodulation of coherent PSK employing MRC (only about half an order of magnitude diffecence in $P_{e}$ performance at high $E_{b} / N_{0}$ values).

A prototype system based on the analysis of [4] has been built and reported upon in [5]. A DS-SSMA system using DPSK equal gain combining diversity was implemented using a SAW filter matched to the user's PN sequence. An M-sequence was used for spreading and had a length of 127 chips and a chip period of 100 nanoseconds. The data rate of the system was $78.7 \mathrm{KHz}$ and only one interferer was tested (i.e. the system was implemented with only two users present). The system was tested in an indoor environment with fades of up to 50dB. When a single interferer was present (both signals were received with equal strength at the rcceiver), the degradation in performance from that of a single user, varied from 0 to $2 \mathrm{~dB}$ depending on the location of the receiver relative to the transmitter.

The notation employed to analyze asynchronous SSMA systems is based on that developed principally by Pursley[12] [13][15][16] (see also [17][18][19]). This notation assumes an asynchronous DS SSMA system employing $K$ users (no fading or multipath). The nathematical details of the notation will be presented in Chapter 2 . Pursley developed gencral expressions for the interuser interference term in a SSMA 
system derived in terms of continuous time partial cross-correlation functions and the aperiodic cross-correlation function $C_{k, i}(l)$. Other related correlation functions are the even and odd cross-correlation functions $\theta_{k, i}(l)$ and $\dot{\theta}_{k, i}(l)$, respectively. In [12] Pursley provides two estimates of system performance based on the aforementioned cross-correlation parameters. The first is a bound on the maximum probability of bit error based on the Gaussian assumption. The second measure of performance of interest is an estimate of the average signal to noise ratio based on the variance of the interuser interference random variable

In a companion paper to [12], Pursley and Sarwate discuss some of the relationships and bounds relating to $\theta_{k, i}(l), \hat{\theta}_{k, i}(l)$ and $C_{k, i}(l)[13]$. In order to evaluate the probability of error rate performance for various modulation techniques Pursley et al. developed several bounds and approximations. Upper and lower bounds on the average probability of error, as well as generalized Chebyshev bounds were derived using a recursive technique[14]. Geraniotis and Pursley also developed two approximation techniques to evaluate the average probability of error for a DS-SSMA system. The first approach employs a characteristic function whereas the second approach involves evaluating a series expansion of the average probability of error (the Gaussian assumption for the interference is invoked). These two techniques were employed to evaluate the performance of BPSK, QPSK, OQPSK and MSK modulated systerms. The spreading sequences investigated were either Cold sequences or $\mathrm{M}$-sequuences (the sozuences were chosen to be auto-optimal with least sidelobe energy), with periods in the range of 31 to 255 chips, whereas the number of users $K$ in the systern model varied between 3 and 24. Yao also evaluated the performance of DS-SSMA systems using the theory of moments[21]. Bounds were developed based on various moments of interuser interference. These include the second moment, the fourt/s moment, the single exponential moment and multiple exponential momornts.

Laforgia et al.[22] also evaluated the bit error rate performance of SSMA systems based on moment generating techniques. Mcre spexifically they crnployed thr. 
method of moments (as was subsequently used by Kavehrad in [2][3]) to evaluate the effects of interuser interference using Gauss-quadrature integration techniques. This technique has been used extensively in the past to evaluate ISI type problems. A good tutorial paper is that due to Meyers[23]. The performance for BPSK, OQPSK, QPSK and MSK DS-SS systems was evaluated using this technique. The results obtained were comparable to those of Geraniotis and Pursley[16].

There have been many other studies in the open literature dealing with the performance of DS-SSMA [25][26][29], as well as papers which deal with error rate performance of SSMA in frequency selective fading channels[27][28]. Geraniot:s examined the performance of binary DS-SSMA in both nonselective and frequency selective Rician fading channels. The system model of [12][13] was employed and the performance was evaluated using a characteristic function approach similar to that used in [16]. Both rectangular and sine pulse shapes were used as chip waveforms.

Ochsner calculated the performance of DS-SS on a frequency selective channel by using Bello's model of a wide sense stationary uncorrelated scattering channel model (WSSUS)[30]. Performance was evaluated assuming ideal PN sequences and assuming a discrete channel impulse response that could be orthogonalized. Based on these assumptions an optimum receiver was derived and its performance evaluated.

\subsubsection{Code Sequence Analysis}

In the previous section it was outlined how the performance of a DS-SSMA system deperds on the correlation properties of the spreading code. Three principal parameters that are employed to describe these properties are the aperiodic cross correlation function $C_{k, i}(l)$, and the even and odd periodic correlation functions $\theta_{k, 2}(l)$ and $\hat{\theta}_{k, i}(l)$. The code sequence must be selected in a manner that will minimize these cross correlation parameters in order to reduce the interuser interference.

There have been numerous texts and papers written on how to generate pseu- 
dorandom sequences and calculate their correlation properties. Two thorough tutorial papers covering these areas are those due to MacWilliams and Sloane[31] and Sarwate and Pursley[32]. The paper by MacWilliams and Sloane provides a good review of how to generate pseudorandom shift register sequences (i.e. in particular M-sequences), from primitive polynomials over a finite Galois Field GF(q), and their properties. The relationship of these codes to classical error correcting coding theory is reviewed (see also the texts by MacWilliams and Sloane[34], Peterson and Weldon[35] as well as Simon et al.[36]), and in addition two-dimensional pseudorandom arrays and non-binary pseudorandom sequences are examined (i.e. with elements from $G F(q), q>2$ ).

One design criterion that can be employed in a SSMA system to reduce interference is to minimize the maximum cross correlation between any two sequences from a set of sequences to be used for SSMA. Welch [37] has derived a lower bound for the maximum value of both periodic and aperiodic cross-correlation functions. These bounds have been elaborated upon by Sarwate[38] for the case appropriate to SSMA.

Besides the peak correlation bounds described above relating to aperiodic crosscorrelation and the even periodic cross-correlation, there are two other parameters that relate to the aperiodic cross-correlation functions that are significant. The first of these is defined as the sidelobe energy[40] which is a mean-square autocorrelation parameter, and is of importance for synchronization and inultipath considerations. Another mean-square autocorrelation parameter of importance is the auto-optimal phase of a given sequence[41].

The bounds derived by Welch and Sarwate give a worst case measure of performance, but provide little information about the average cross-correlation parametcers of the set of sequences to be employed. Pursley and Roefs have defined an averagu: interference parameter $r_{k, i}[12][40]$. Also see [41] in which a bound on the maximum mean square cross-correlation for a set of restricted signals is derived. 
The previous discussion centered upon the properties of, and the bounds of the cross-correlation of a general set of finite sequences. Much research has been spent investigating the cross-correlation properties of specific sets of binary sequences. $M$ sequences are well known for their close to ideal autocorrelation properties (for an $\mathrm{M}$-sequence of length $\mathrm{N}$, the autocorrelation with zero phase shift is $\mathrm{N}$, whereas it is -1 at all other phase shifts), however the number of different M-sequences of a given length, with small values of cross-correlation is restricted.

Trading off some of the nearly ideal autocorrelation of $\mathbf{M}$-sequences for improved cross-correlation, one can obtain sets of well known binary sequences such as Gold codes [32][42][43] [44] or Kasami codes[32][45]. Gold codes of length $N$ are generated by use of a polynomial (i.e. using a shift register generator), which is the product of two properly selected primitive polynomials each of length $n$ (the code is of length $N=2^{n}-1$ ). Kasami codes are superior to Gold codes in cross-correlation performance, but are fewer in number for a given sequence length.

There are several other classes of binary sequences with similar cross-correlation properties to Gold and Kasami sequences, as described by Pursley and Sarwate[32]. These include the large set of Kasami codes, Dual BCH sequences and sets of reciprocal M-seq :ences.

Studies of the cross-correlation properties of sequences whose elements are chosen from GF( 4 ), q prime and greater than 2 (i.e. nonbinary), have also been carried out. $A$ maximal linear sequence of length $q^{n}-1$ can be generated from a primitive polynomial of order $n$, with coefficients taken from GF(q). See for example Peterson and Weldon[35] or Macwilliams and Sloane[34] for details on how to generate such sequences given a primitive polynomial. Algorithms to generate the required primitive polynomials are available ( see for example Simon et al. [36] Appendix 5), and tables of such polynomials for $q$ prime are also available[46][47][48][52].

llaving obtained sequences in higher order alphabets, one must choose a mapping into a usable signal format. One such mapping that has been investigated is to map 
the sequence over $\mathrm{GF}(\mathrm{q})$ into a complex roots of unity sequence. Roots of unity sequences have the desirable property (i.e. for synchronization) that for sequences of length $N$, the in phase autocorrelation is $N$, whereas it takes on a value of $-l$ for all other phases. Many theorems regarding the values taken on by the crcss-correlation function of roots of unity sequences, and the number of times these values occur, have been derived. One of the principal techniques used to evaluate these cross-correlation functions is through use of the "trace function". Details of the trace function can be found in Simon et al.[36] or in MacWilliams and Sloane[31]. Helleseth has used this technique to evaluate numerous cross-correlation properties of roots of unity sequences[49].

Specific st'ndies of the correlation properties of nonbinary sequences over GF(q) include those by Briggs and Godfrey[50], Godfrey[51], Balza et al.[53], and Chang[54]. These papers deal with the investigation of the autocorrelation functions of three and four level M-sequences. The paper by Godfrey[51] deals with 3-level M-sequences (i.e. over $\mathrm{GF}(3)$ ) and the autocorrelation function of the resulting sequences (these properties are generalizations of the well known properties of binary M-sequences). The papers by Briggs and Godfrey[50] and that of Balza et al.[53] deal with properties of 4 level M-sequences such as their autocorrelation properties and spectral density. In fact these sequences can be represented in an equivalent binary sequence form by noting that $G F(4) \equiv G F\left(2^{2}\right)$ (i.e. see [36] - Vol. I Appendix 5). Also, the paper by Chang investigates some properties of 5 -level $\mathbf{M}$-sequences.

Other relevant investigations include those of Scholtz and Welch[55] who studiry the correlation properties of sequences of "group characters", Sivaswany[56] who studied the properties of multiphase complementary codes (MPC), Shaar at al[57] who investigated the performance of and derived upper and lower bounds on the cross-correlation properties of "state $M$-sequences", as well as papers related to cordo sequences by McEliece[58] and by Pursley and Sarwate[59]. 


\subsubsection{Trellis Coding}

The original work on trellis codes is attributed to Ungerboeck[6]. Using channel capacity arguements Ungerboeck demorstrated performance gains could be obtained using multilevel/phase constellations. Classical coding theory achieves coding gain by maximizing the Hamming distance between any two possible codewords. In constrast Ungerboeck maximized the Euclidean distance between any two possible received signal sequences. This was accomplished through "mapping by set partitioning". Typically a block of $m$ bits was coded using a binary convolutional coder of rate $R=m / m+1$ bits. The " $m+1$ " encoded bits were then mapped into a channel signal set in a manner that maximized the Euclidean distance between any two code words. The most difficult aspect of defining the code was in fact mapping the convolutionally coded sequence into the chosen signal set. The first aspect of the mapping was to divide the signal set into subsets such that the minimum Euclidean distance between any two points in the subset exceeded that of the original set. The subsets themselves were then subdivided in the same manner, with the partitioning process continuing until no further subdivisions could be obtained. As an example. an 8-PSK constellation can be subdivided into 2 QPSK constellations, which can be further subdivided into four BPSK constellations. Depending on the number of states in the convolutional code, the signal subsets were mapped into the states of the selected code in order to maximize the free Euclidean distance.

Ungerboeck found trellis codes which maximized $d_{\text {free }}$, through what were basically exhaustive searches of all possible codes. For a given signal set and partitioning of that signal set, Ungerboeck developed an algorithm to check all possible relevant combinations of parity check coefficients of the convolutional code portion of the trellis code based on the concept of Euclidean weights.

Since Ungerboeck's original paper (described above), there has been a significant amount of research in the field of trellis coding. A good tutorial survey of much of this work was undertaken in a two article set by Ungerboeck[60][61]. The first 
article provides a tutorial description of the concepts of [6], whereas the second article provides an overview of recent advances in trellis coded modulaton (TCM). These advances include a new description of TCM[62] as well as the development of a general encoder structure[7]. Furthermore, a mathematical basis for the seloction of trellis codes based on the theory of lattices and sphere packings has been formalized[7][63][64]. Other studies have investigated rotationally invariant trellis codes[65][66] and multidimensional trellis codes(67)[68]. In [6] Ungerboeck tabulates the parity check codes for some of the best known trellis codes for given signal sets.

Another earlier tutorial survey of modulation and channel coding (which includes an overview of trellis coding), is that due to Forney et al.[70]. This paper provides an overview of the optimum selection of uncoded signal constellations, a review of mapping by set partitioning, and how this concept can be applied to both block codes as well as convolutional codes (i.e. trellis codes). An alternate description of trellis coding has been developed by Calderbank and Mazo[62].

A more recent work by Calderbank and Sloane presented a more general description of trellis codes[7]. In their formulation of trellis codes, a sequence of symbols from an input alphabet $A$ of size " $a$ " (i.e. if the alphabet is chosen over $G F(q)$, then $a=q$ ) is mapped into a sequence of output points taken from a lattice $\Lambda$ in $\mathrm{n}$-dimensional Euciidean space $R^{\mathrm{n}}$. The set of all output points forms a constellation which is a finite subset of $\Lambda$. The authors identified three necessary romponents (besides $\Lambda$ ) that are required in order to define the trellis code. The first of these is a sublattice $\Lambda^{\prime}\left(\Lambda^{\prime} \subseteq \Lambda\right)$. It is necessary that $\Lambda$ and $\Lambda^{\prime}$ be selected such that $\Lambda$ is an Abelian group and that $\Lambda^{\prime}$ is a subgroup. The second aspect of the trellis code requires that $k_{1}$ of $k$ input symbols enter a convolutional coder, the output of which selects a coset of the sublattice $\Lambda^{\prime}$. Formally, the output is an element of $R=\Lambda / \Lambda^{\prime}$ in which $R$ is a factor or quotient group (i.e. the group of cosets of $\Lambda^{\prime}$ ). Defining $k_{2}=k-k_{1}$, the remaining $k_{2}$ input symbols select one of the $a^{k_{2}}$ possible points in a coset. Thus a set of $a^{k_{2}} \cdot T$ (T is the number of cosets a vailable - i.e. the order of $R$ ) 
signal points is required. The performance of the code is determined, as discussed previously, by the minimum squared Euclidean distance between output sequences corresponding 10 distinct input sequences. This minimum distance is the minimum of two possible values: (i) the minimum distance $d_{1}$ between output sequences of the convolutional coder (i.e sequences of cosets), and (ii) the minimum Euclidean distance $d_{2}$ between points in any coset (note that these signal points are uncoded). Thus the designer should attempt to choose a convolutional code such that $d_{1} \doteq d_{2}$ (having $d_{1}$ exceed $d_{2}$ gains little). The authors examine in detail several examples of the effects of combinations of signal constellations, lattices and convolutional codes on the resulting performance of the given modulation scheme. Furthermore. a comparison of most known trellis codes (i.e. such as those of Ungerboeck. Calderbank and Sloane, etc.) is provided in tabular form. Parameters that are relevant in the comparison are the rate of the code (bits/dimension). the number of states in the trellis code, the path multiplicity (i.e. the number of paths in the trellis at minimum distance $d_{\text {free }}$ from the correct path) and the convolutional code that was used.

As mentioned previously, an in depth exposition of the use of coset codes and lattices in trellis codes has been undertaken by Forney[63][64]. The first paper[63] deals principally with the gains that can be obtained by selecting various lattices in conjunction with appropriate partitions that produce desired sublattices. Forney deals with the lattices from a mathematical basis and defines various parameters of lattices that are germane to the discussion of trellis code performance.

After defining the mathematical terminology, Forney discusses various implementation strategies of the coder used for coset selection., an: well as various well known lattices and partitions of these lattices. Block coders for coset selection are discussed (particularly the use of Reed Muller codes - see also Cusack[71]), as well as convolutional coders (i.e. the analysis is general enough to accommodate both block and convolutional codes). Extensive tables are provided of trellis codes (classified according to type, such as Ungerboeck. coset. etc.) and then reclassified according to 
gain (1.5dB, 3dB. 4.5dB, and 6dB). Codes are compared based on the type of lattice and sublattice, the order of the partition, the number of states in the coder (for convolutional codes), the number of information bits $k$, the number of redundant bits ' $\rho$ ', the minimum distance, $N_{0}$, and $N_{D}$ a measure of decoding complexity.

A companion paper[64] is a detailed treatise of multi-dimensional lattices, as well as the codes and decoding methods that can be used in their implemeniation. Forney identifies several construction techniques which enable one to create higher dimensional lattices by selected partitions of lower dimensional lattices (leading to lattices with ircreased density for the same value of $d_{\min }$ ). Two of these techniques are the "squaring construction" (for lattices of dimension $N=2^{n}$ ), and the "rubing construction" (for lattices of dimension $N=3 \times 2^{n}$ ). Also explored are properties of the $(24,12)$ Golay code, the 24-dimensional Leech lattice and efficient iterative decoding algorithms for lattice codes.

There have also been numerous other papers detailing the mathematical properties and structures of lattices, most notably papers authored or co-authored by Sloane[72][73][74] [75j[76][77j $\left.{ }_{i}^{78}\right][79]$. There has also been significant effort put into finding efficient decoding algorithms for lattices and lattice codes[80]|[81] [82] [83]. Ipper bounds on the performance of trellis codes have been established by (alderlank ei al.[84][85] (see also [86]).

The performance of specific trellis codes can be evaluated using a modified form of the transfer function bound usually employed to evaluate the distance properties and performance of conventional convolutional codes[87][88][89]. The conventional approach employs 1 generating function of the form $T(D, L, I)$, in which I), L, and I are indeterminate variables whose exponents indicate the Hamming weight or distance of a path (i.e. from the all zeros path), the length of the path (i.e. the numixer of branches in the path), and the number of bit errors caused by the incorrect path, respectively[91][92]. However, since in general a trellis code is nonlinear, the distance between any incorrect path and a correct path is dependent on the corrert 
path. In general. one will have to solve a transfer function with $m=2^{2 M}$ states (see $[88][89][90][93])$ in which $M$ is the encoding constraint length of the code. This is equivalent to solving a $\mathrm{m}^{2} \times \mathrm{m}^{2}$ matrix to evaluate $T(D, L, I)$. If the trellis code and signal constellation possess sufficient symmetry, the size of the transfer function bound can be appropriately reduced (see also [87] for an alternative method).

Besides the above studies which have explored the general performance of trellis codes, there have been numerous other studies investigating various examples and variations of trellis codes[92-104]. One area that has received attention is the study of multidimensional trellis code implementions. Calderbank and Sloane[68] as well as Gersho and Lawrence [67] have investigated 4-D QAM schomes. A paper by Wei[69] also investigates multidimensional trellis codes.

IVei also studied the tolerance of trellis code schemes to $180^{\circ}$ and $90^{\circ}$ phase ambiguities in a set of companion papers[65][66]. Divsalar and Simon studied the effects of varying the asymmetry angle between the in-phase and quadrature components of the signal constellatiun in question. Gains of $1 \mathrm{~dB}$ to $3 \mathrm{~dB}$ were achieved over the performance of the symmetric case.

Pottie and Taylor [95] have investigated va: :ous bounds for trellis codes[86][95] as well as a generalized set partitioning scheme[96]. Also of note are works on asymmetric trellis coding[88]. trellis coding in a partial response channel[97], and multiple trellis coded modulation (MTCM) [103].

Recent investigations have also examined the performance of trellis codes in fading channels [104][105][106][114] [115][116]. One of the major results of these studies is that maximizing the free Euclidean distance is not the optimum criterion for selecting trellis codes in a fading environment. Simon and Divsalar [104] demonstrated that the best criterion in a Rayleigh fading environment was to maximize the length of the shortest path error event. If the channel was Ricean. the selection of the best trellis code involved maximizing both the shortest path error event and $d_{\text {free }}$ The results of these preliminary studies indicated that significant gains could be 
obtained by the use of trellis coding in fading environments. However if fast fading was present, or the fading process was strongly correlated over the constraint length of the decoder, then interleaving was required to help mitigate the efferts of the fading.

\subsection{Thesis Organization}

The general background of the study as well as an overview of the relevant papeers in the open literature have been presented in this introduction. Chapter 2 describes the system model to be employed in the anslysis as well as a derivation of tha. notation to be employed in subsequent analysis. Chapter 3 provides an overview of the generalized transfer function bound that has been employed to evaluate the performance of the coded systems under consideration. Detailed examples of the transfer function bound derivation for some of the specific codes studied are providerl. Chapter 4 derives expressions of the Chernoff upper bound for the pairwise error in the performance of convolutional and trellis codes, and the performance of the . codes in an AWGN channel is presented in Chapter 5. Chapter 6 derives the ( hernulf upper bounds on the performance of both trellis and convolutional codes in a ltician fading environment both with and without channel state information. ('hapter 7 presents the performance results for the Rician fading channels. (Chapter $x$ presents the conclusions of the thesis as well as suggestions for future research.

Appendix A provides a derivation of the general 6 :pression for the mome.nts of the. interuser interference required in the method of moments. An alternate derivation of the moments is provided in Appendix D. Appendices $B$ and $\left({ }^{\circ}\right.$ derive expressions re. lated to the Rician distribution, whereas Appendices $\mathrm{E}, \mathrm{F}$ and $\mathrm{G}$ provide derivations of other relevant moment expressions. 


\subsection{Thesis Contributions}

The Inajor contribution of this thesis is that a system model is proposed and analyzed that allows one to apply trellis coding to an asynchronous SSMA system. The performance of such systems employing rate 1/2 4-PSK, rate $2 / 3$ 8-PSK, and rate $3 / 4$ 16-PSK trellis codes (with up to 16 states) has been evaluated in both the presence of AWGN alone and the combination of AWGN and Rician fading. The performance in Rician fading channels has been determined both with and without channel state information. These results are new. In order to benchmark the performance of the trellis coded system, the performance of convolutionally coded SSMA systems has also been evaluated using equal throughput and complexity as criteria of comparison. The convolutional codes that have been considered are rate $1 / 2,1 / 8,2 / 3$ and $2 / 7$ codes, with up to 16 states. The performance of convolutionally coded SSMA systems has been previously estiuated by employing a Gaussian approximation for the SSMA interference[33]. The results in this thesis provide an exact confirmation of the performance of the convolutionally coded SSMA systems. The comparison of the trellis and convolutional codes demonstrates that the best performance (for the codes considered) is obtained from low rate convolutional codes.

The validity of the use of the Gaussian approximation for the SSMA interference has also been calculated both for the trellis and the convolutionally coded systems. It will be shown that this approximation is overly optimistic, which is consistent with the conclusions of previous studies in SSMA channels[3]. 


\section{Chapter 2}

\section{System Model}

\subsection{General System Architecture}

The general architecture of the system that will be analyzed is illustrated in Figure 2.1, which has been used frequently in previous studies of asynchronous $\operatorname{SiMA}$ systems[2][12][15]. The system is comprised of $K$ users transmitting asynchronously over an Additive White Gaussian Noise (AWGN) channel. Each user transmits using a different spreading code and the signal transmitted by the $k^{\text {th }}$ user $s^{k}(t)$ is assumed to be delayed randomly by a delay of $\tau^{k}$. Thus if the roceiver of ther $i^{\text {th }}$ uscr is attempting to receive the signal transmitted by the $j^{\text {th }}$ user, the received demend. ulated signal will consist of the desired signal and interference due to a combination of the AWGN and the signals transmitted by the other users on the system. The notation required to analyse the performance of such a system will be developed in section 2.2. The interferers are all assumed to arrive at the desired receiver with equal signal powers. A final general point is that protocols for addressing other users or for transmitting and receiving signals are not considered in this study. 


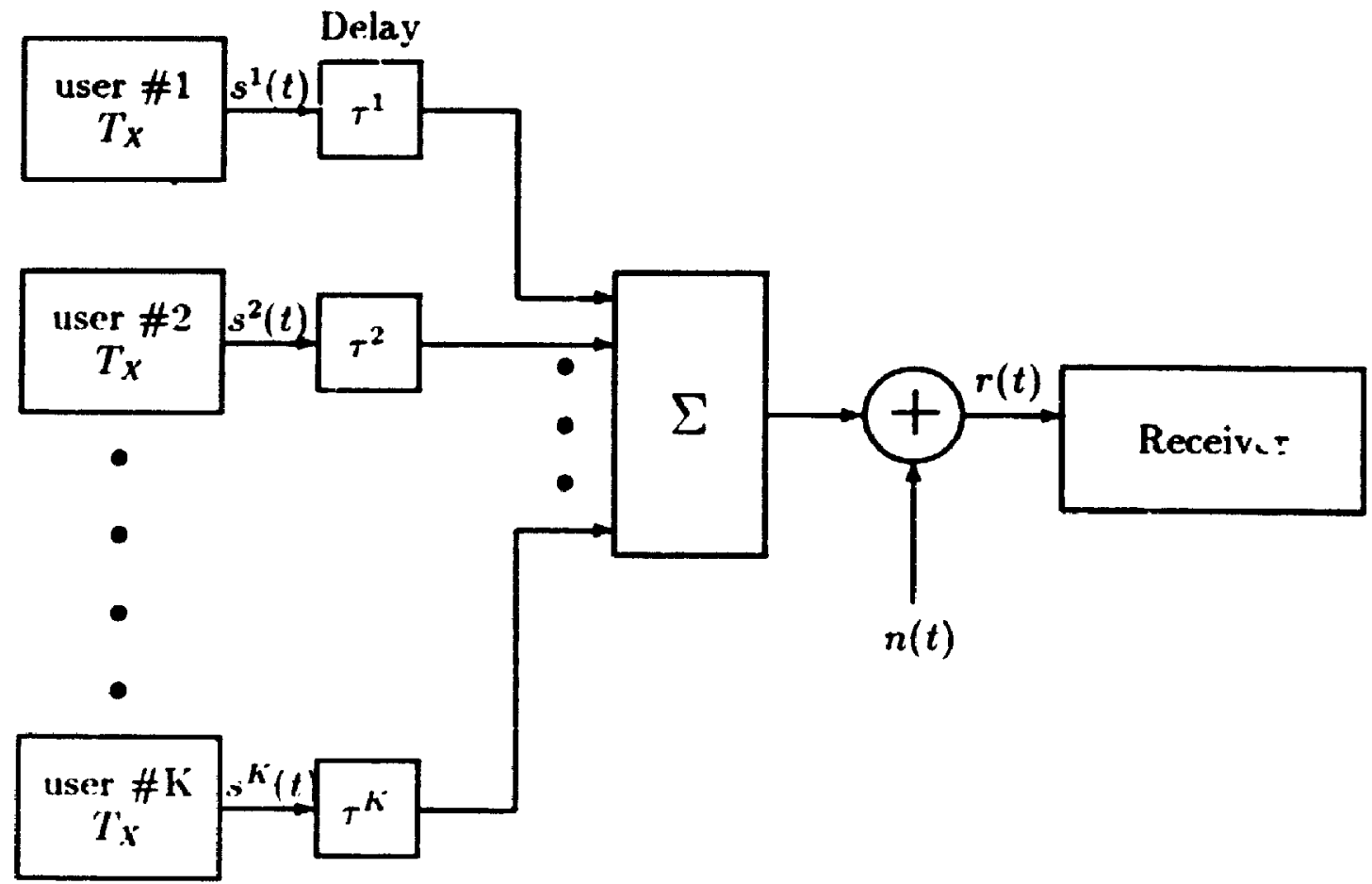

Figure 2.1: System model. 


\subsubsection{Trellis Coded SSMA}

The transmitter system model for trellis coded SSMA is illustrated in Figure 2.2a. This architecture is based on that defined by Calderbank and Sloane [i], wherelby a trellis code is based on the combination of a lattice $\Lambda$, a sublattice $\Lambda^{\prime}$, a convolutional code, and a signal constellation. The signal constellation is a subset of the lattice $\Lambda$ and the convolutional code selects one of the cosets of the sublattice $\Lambda^{\prime}$. For $k$ input bits from the data source, $k_{1}$ of these bits select the coset of $\Lambda^{\prime}$ through the convolutional coder. while the remaining $k_{2}$ select the signal point within the given coset. The architecture of Figure $2.2 \mathrm{a}$ is analogous to the above description, with the additional feature that the phase of the signal point chosen within the given coset is multiplied by the PN spreading sequence to be transmitted in the given symbol period (it is assumed that the data symbol transitions are aligned with chip transitions, so that there is no spectral spreading due to the multiplication process). Thus in effect, what is achieved is that in each symbol period, a binary SS signal is transmitted, the absolute phase of which is determined by the trellis codr. 'The two antipodal signal points (denoted as the antipodal coset) employed by the PN sequence as well as the modulation on the PN sequence is selected by the 1 rellis coder - see section 2.2 for further details.

In more conventional terms, the process of selecting a sublattice from a lattice is equivalent to using a redundant alphabet (i.e. the signal points in the constollation that can be transmitted), and partitioning the alphabet (or constellation) into subsets with increasing Euclidean distance. This partitioning process for an $x-\mathrm{p} S \mathrm{~K}$ signal constellation is illustrated in Figure 2.3 (see [6]). Such a constellation wonld be employed, for example, with a rate $2 / 3$ trellis code. If this code was cmployed in a SSMA system, in any particular symbol interval an antipodal PN serjuence is transmitter, the absolute phase of which is one of $\pm \pi / 8, \pm 3 \pi / 8, \pm 5 \pi / x$, or $\pm \pi \pi / x$. The antipodal cosets for 4-PSK, 8-PSK and 16-PSK signal constellations a ro illustrated in Figures 2.4. 2.5 and 2.6. Also labelled in these figures are the iturlirlenu 
a) Trellis coded baseband system:

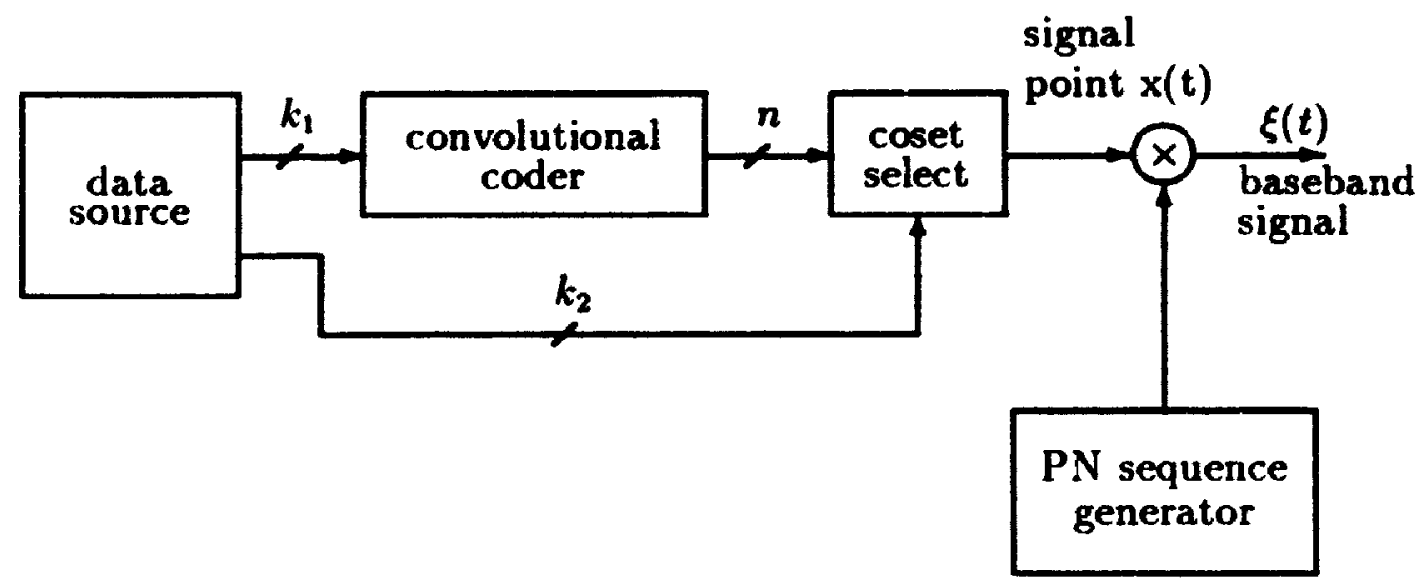

b) Convolutionally coded baseband system:

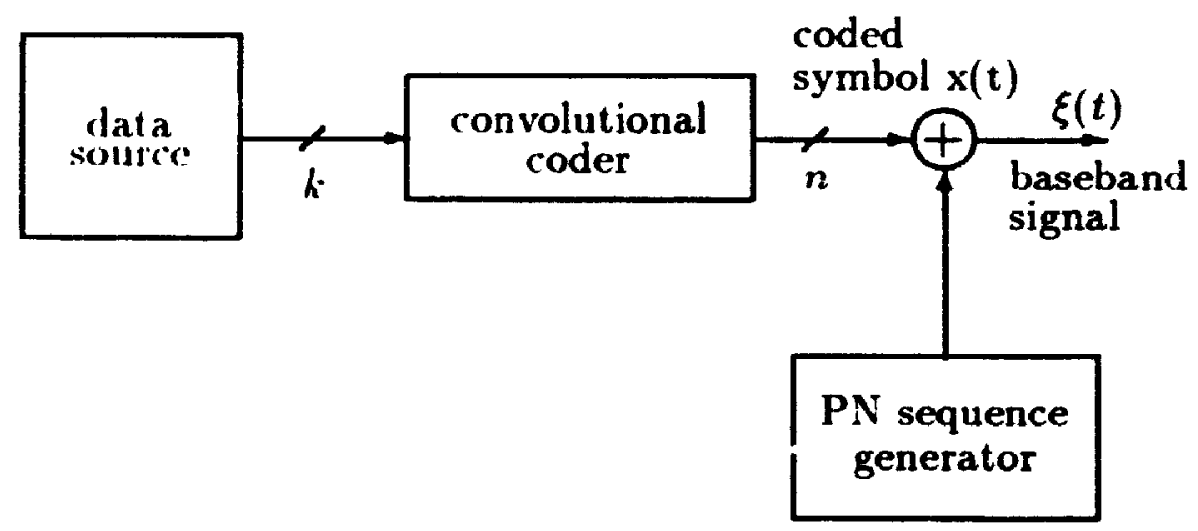

Figure 2.2: Generalized transmitter baseband system model. 


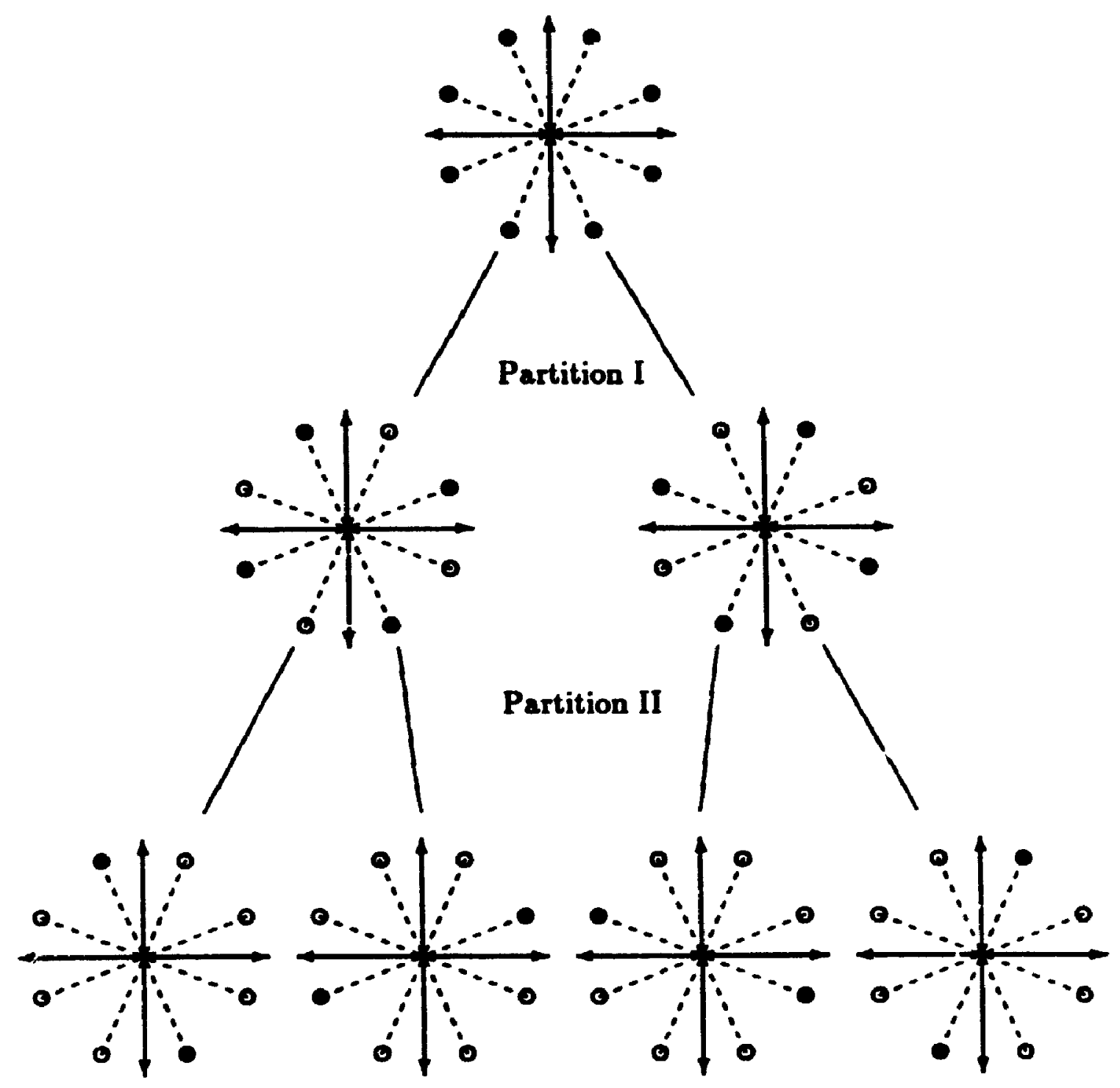

Figure 2.3: Partitioning of 8-PSK signal constellation. 


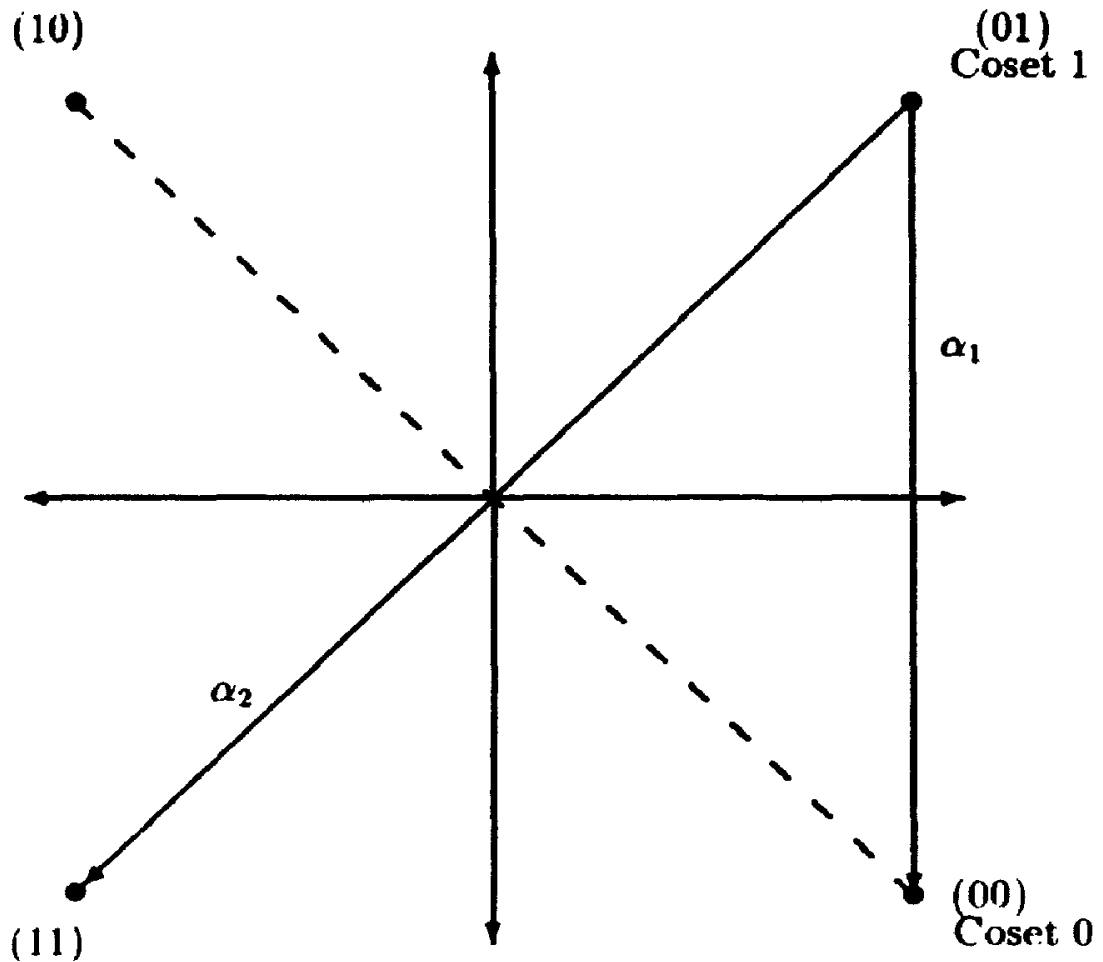

Figure 2.1: Antipodal cosets and Euclidean distances of 4-PSK 


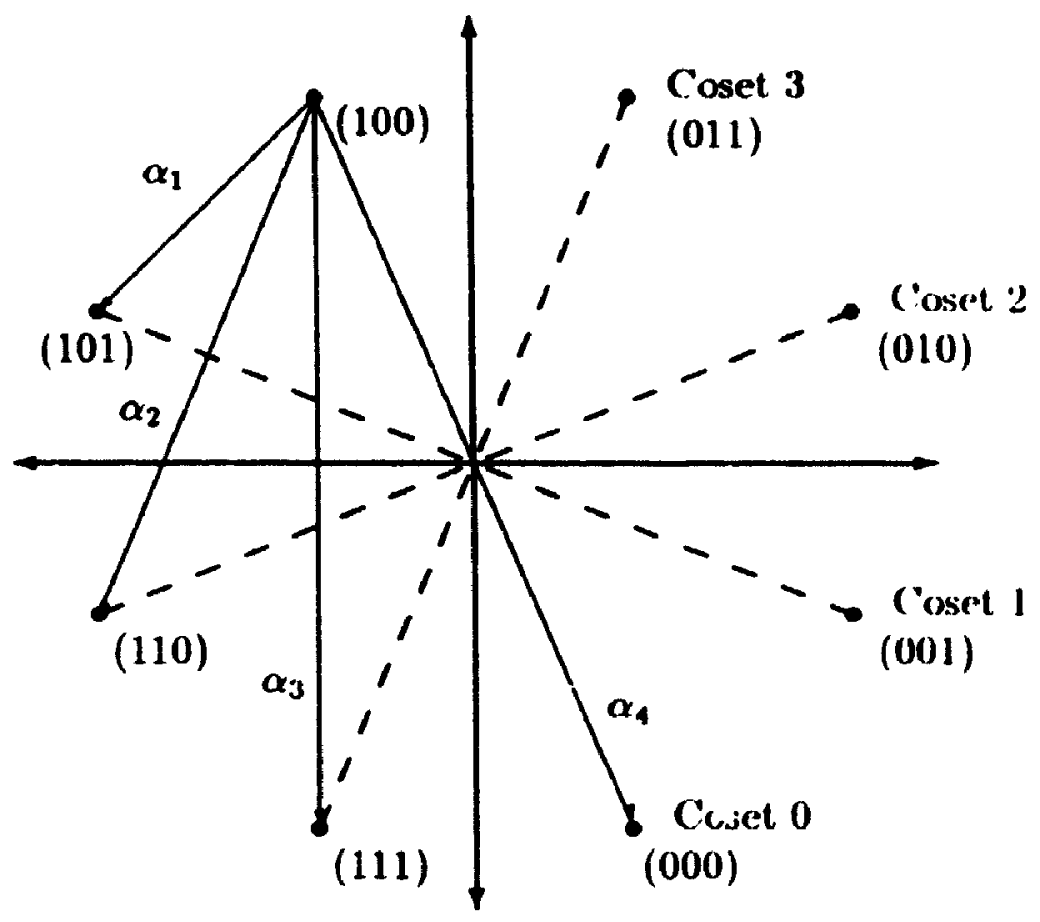

Figure 2.5: Antipodal cosets and Euclidean distances of 8-PSK. 


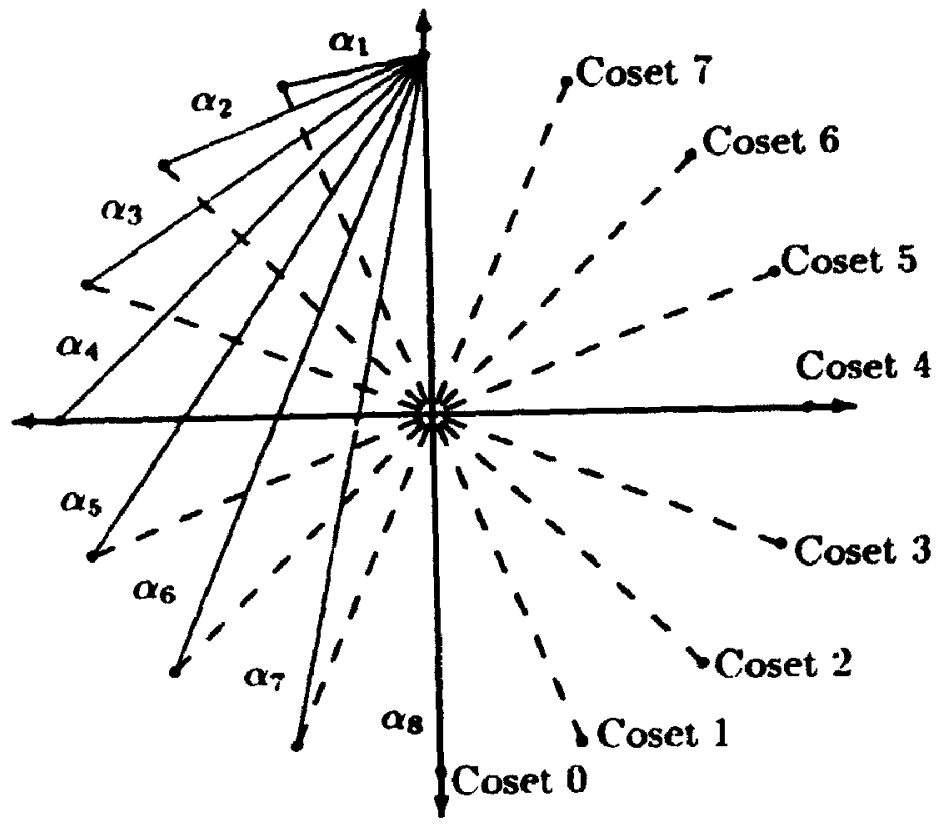

Figure 2.6: Antipodal cosets and Euclidean distances of 16-PSK. 
distances between signal pointe which are tabulated in Table 2.1.

For codes which have parallel transitions between any two states of the trellis. each of the branches in the parallel transition pair can be assigned a signal point in the antipodal coset. This allows a somewhat simpler implementation of the baseband architecture. The convolutional code portion of the trellis code would select the antipodal coset to be used in the transmisaion (i.e. from the $k_{1}$ source bits), whereas the $k_{2}$ uncoded source bits could be modulo-2 added to the PN sequence. The resulting data modulated sequence would then be transmitted using the signal points selected by the convolutional code (i.e. the antipodal coset). Examples of such a code are the rate 1/2, 2-state 4-PSK trellis code and the rate 2/3, 4-state 8-PSK trellis code. In general except for certain low complexity codes, such as those described above, both the antipodal coset and the data modulation on the PN sequence to be transmitted are selected by the trellis coder. An example of a trellis diagram is provided in Figure 2.7 for the rate 1/2 4-state 4-PSK code.

\subsubsection{Convolutionally Coded SSMA}

Figure 2.2b illustrates the baseband transmitter system model employed to analyze the performance of convolutionally coded SSMA systems. In this model the data source produces a group of $\boldsymbol{k}$ data bits, which enter a rate $\boldsymbol{k} / \boldsymbol{n}$ convolutional cuder. Each of the $n$ coded symbols output by the convolutional coder is then modulaterd directly onto the PN spreading sequence using a BPSK signalling format. The coded symbol can modulate the PN sequence in one of two possible manners. One approach is to have each coded symbol span one complete period of a $P N$ sequence. If it is assumed that the convolutional code is to have a throughput equal to that of a trellis code of the same complexity and that the chip period is fixed, then it follows that the length of the PN sequence must be shortened by a factor of $1 / n$. $T^{\prime}$ is results in additional degradation due to interuser interference since it is well kr. swn that the cross-correlation between any two PN sequences is proportional to $\frac{1}{N_{c}}$ in which $N$. 


\begin{tabular}{|c|c|c|c|}
\hline \multirow{2}{*}{ parameter } & \multicolumn{3}{|c|}{ Squared Euclidean Distance } \\
\cline { 2 - 4 } & $4-$ PSK & $8-$ PSK & 16-PSK \\
\hline$\alpha_{1}$ & $2 E_{0}$ & $2 E_{0}\left(1-\frac{1}{\sqrt{2}}\right)$ & $2 E_{0}\left(1-\sqrt{\frac{\sqrt{2}+1}{2 \sqrt{2}}}\right)$ \\
\hline$\alpha_{2}$ & $4 E_{0}$ & $2 E_{0}$ & $2 E_{0}\left(1-\frac{1}{\sqrt{2}}\right)$ \\
\hline$\alpha_{3}$ & - & $2 E_{0}\left(1+\frac{1}{\sqrt{2}}\right)$ & $2 E_{0}\left(1-\sqrt{\frac{\sqrt{2}-1}{2 \sqrt{2}}}\right)$ \\
\hline$\alpha_{4}$ & - & $4 E_{0}$ & $2 E_{0}$ \\
\hline$\alpha_{5}$ & - & - & $2 E_{0}\left(1+\sqrt{\frac{\sqrt{2}-1}{2 \sqrt{2}}}\right)$ \\
\hline$\alpha_{6}$ & - & - & $2 E_{0}\left(1+\frac{1}{\sqrt{2}}\right)$ \\
\hline$\alpha_{7}$ & - & - & $2 E_{0}\left(1+\sqrt{\frac{\sqrt{2}+1}{2 \sqrt{2}}}\right)$ \\
\hline$\alpha_{0}$ & - & - & $4 E_{0}$ \\
\hline \hline
\end{tabular}

Tabie 2.1: Squared Euclidean distances between constellation signal points 


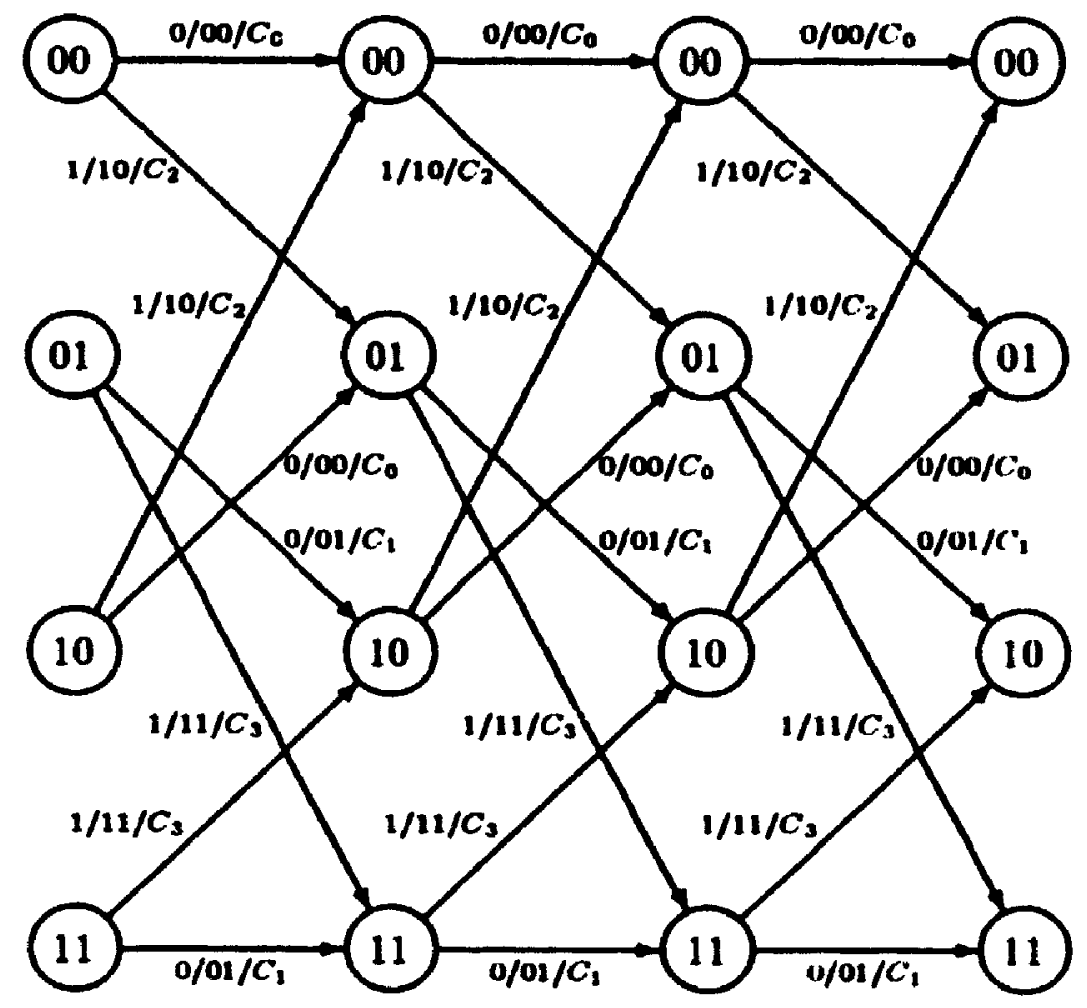

transition labels: $a / b / c$

$$
\begin{aligned}
& a=\text { input bit } u_{0} \\
& b=\text { output } \mu_{0} \mu_{1} \\
& c=\text { coset }
\end{aligned}
$$

Figure 2.7: Trellis state diagram of rate 1/2 4-state 4-PSK trellis rede. 
is the number of chips in the PN sequence[32].

An alternative implementation of a convolutionally coded system would be to have each coded symbol span a fraction $\frac{1}{n}$ of the PN sequence period. However this will also result in increased degradation due to the partial cross-correlation properties of the desired signal with the interfering signal. In general, the partial cross-correlation of two sequences is larger than the cross-correlation of the ce mplete sequences[20]. The tradeoff between the greater distance properties of lower rate codes and the increased cross-correlation effects of employing lower rate codes will be apparent in the results presented in Chapters 5 and 7.

\subsubsection{Decoding}

Nearly optimal decoding of the above type schemes can be achieved by employing the Viterbi algorithm (the decoding is not optimal since the correlator receiver is matched to the AWGN in the channel, and not to the combination of the AWGN and the interuser interference). At the receiver, ite received signal is correlated with the PN spreading sequence for each of the possible antipodal cosets in the code. This can be accomplished by correlating the received signal with one of the signal points in the antipodal coset. since the value of the correlation for any two signal points in the same antipodal coset will have the same magnitude but opposite polarities. In general. for cach coset, the estimate of the data bit modulated on the PN sequerice is stored, as well as the distance of the received signal from the coset (or any other mitric uscd in the decision process). The Viterbi algorithm is used to identify the signal path (i.e. the sequence of cosets) through the code trell:s which has minimum distance from the rereived signal sequence (see section 2.2 for more details). 


\subsection{Derivation of Complex Interuser Interference}

The following notation is based on that developed by Pursley [12][15] and utilized by Kavehrad in his analysis of SSMA for indoor wireless radio[1][2]. This notation is based on the architecture that was briefly described in Section 2.1. and illustraterl in Figure 2.1. Assume a SSMA system consisting of $h$ users each utilizing a l'N sequence of length $N_{c}$ chips, which spans one data symbol (the effects of the PN sequence spanning more than one data or coded symbol will brexamined near the end of this section). Define the $k^{t h}$ user's complex baseband information signal at the output of the trellis coder or convolutional coder as

$$
x^{k}(t)=\sum_{p=-\infty}^{\infty} x_{p}^{k} P_{\gamma}(t-p T)
$$

in which $T$ is the symbol period and $P_{T}(\cdot)$ is a rectangular pulse of $T$ seromls duration. The term $x_{p}^{k}$ is the complex baseband symbol of the $k^{\text {th }}$ user during the $p^{\text {th }}$ symbol period, defined by

$$
x_{p}^{k}=b_{p}^{k} \exp \left[j \theta_{p}^{k}\right]
$$

in which $b_{p}^{k}$ and $\theta_{p}^{k}$ are the amplitude and phase of the complex baseband output and $j=\sqrt{-1}$. Note that for the convolutionally coded system that the signial constellation is always a BPSK format, sc that equations (2.1) and (2.2) anl l,. simplified to

$$
b^{k}(t)=\sum_{p=-\infty}^{\infty} b_{p}^{k} P_{T}(t-p T)
$$

in which $b_{j}^{k}$ is a coded symbol of the convolutional code which can take on a value of +1 or -1 . The term $b^{k}(t)$ has been used in place of $x^{k}(t)$ of cyuation $(2.1)$ since the. output symbols only take on real values for the convolutionally corled case. Since thre convolutionally coded SSMA implementation is architreturally a simplified versian of the trellis coded case, the subsequent analysis will refer only to the trellis codrad system. If need be, with a simple modification of equation (2.1). the analysis ran br applied to the convolutionally coded SSMA sysu.m. 
Similar to the definition of $x^{k}(t)$, the DS spreading chip waveform is defined as

$$
a^{k}(t)=\sum_{m=-\infty}^{\infty} a_{m}^{k} \psi\left(t-m T_{c}\right)
$$

in which $a_{m}^{k}$ is the $m^{\text {th }}$ chip of the $k^{\text {th }}$ user, $\psi(\cdot)$ is the chip waveform, and $T_{\varepsilon}$ is the duration of the chip pulse with $N_{c}=T / T_{c}$ being the period of the PN sequence. In the above and subsequent definitions, the superscripts refer to the user under consideration, whereas the subscripts denote the time interval under consideration (i.e. a particular symbol or chip). The sequence $a^{k}(t)$ is multiplied with $x^{k}(t)$ (as was mentioned previonsly the data and chip symbol transitions are assumed to be aligner), and the resulting sequence is used to biphase modulate a carrier of frequency $f_{c}$. The resulting transmitted signal of the $k^{\text {:h }}$ user is

$$
\begin{aligned}
r^{k}(t)= & \sqrt{\frac{2 E_{s}}{T}} \operatorname{Re}\left\{\xi^{k}(t) \exp \left[j\left(\omega_{c} t+\phi^{k}\right]\right\}\right. \\
= & \sqrt{\frac{\mathscr{V E}_{s}}{\Gamma}}\left\{\operatorname{Re}\left[a^{k}(t) \cdot x^{k}(t)\right] \cos \left(\omega_{c} t+\phi^{k}\right)-\right. \\
& \left.\operatorname{Im}\left[a^{k}(t) \cdot x^{k}(t)\right] \sin \left(\omega_{c} t+\phi^{k}\right)\right\}
\end{aligned}
$$

in which $\xi^{k}(t)$ is the complex l,aseband signal of the $k^{\text {th }}$ user, defined by

$$
\xi^{k}(t)=a^{k}(t) x^{k}(t)
$$

and $s^{k}$ is the random phase of the $k^{\text {th }}$ carrier. $A$ point of note is that this architecture in effert uses the same PN sequence for both the in-phase and quadrature channels. which differs from Pursleys[15] technique of employing a different PN sequence on ach of the $I$ and $Q$ channels. Examining equations (2.2) and (2.6) it can be seen that the trellis coded SSMA system transmits a biphase modulated PN sequence. the phase $\theta_{p}^{k}$ and amplitude $b_{p}^{k}$ of which are determined by the signal point selected by the trellis code. The binary PN sequence is transmitted using the signal point $x_{p}^{k}$ and the signal point antipodal to $x_{p}^{k}$. Furthermore $E$, is the symbol energy defined by

$$
E_{s}=\int_{0}^{T}\left[s^{k}(t)\right]^{2} d t
$$


The superscript has been omitted from $E$, without loss of generality, since it, is assumed that the symbol energy from one user to the next is constant. Assuning an AWGN channel, the received signal may be expressed as

$$
r(t)=\sqrt{\frac{2 E_{s}}{T}} \operatorname{Re}\left\{\sum_{k=1}^{K} \xi^{k}\left(t-T^{k}\right) \exp \left[j\left(\omega_{c} t+\beta^{k}\right)\right]\right\}+n(t)
$$

in which $\tau^{k}$ is the random delay of each user's signal arriving at the receiver. and

$$
\beta^{k}=\phi^{k}-\omega_{c} T^{k}
$$

is the random phase of the $k^{t h}$ user's carrier at the receiver. Furth more $n(t)$ is a sample of $A W G N$ with variance $\Lambda_{0} / 2$.

For the purposes of analysis the receiver for the $i^{\text {th }}$ user can be implententerl as two correlators matched to the orthonormal carriers[15][91]

$$
\Phi_{1}^{\prime}(t)=\sqrt{\frac{2}{T}} a^{2}(t) \cos \left(\omega_{c} t\right)
$$

and

$$
\Phi_{2}^{i}(t)=\sqrt{\frac{2}{T}} a^{i}(t) \sin \left(\omega_{c} t\right)
$$

which has the form of a conventional I and $Q$ type demodulator. Thus the recriver consists of a correlator matched to the $i^{\text {th }}$ user's PN sequence for both the in-phase' (I), and quadrature (Q) channels (sce Figure 2.8) followerd by a coset correlator which provides inputs to the Viterbi decoder. This receiver is optimum in a: AWC:N environment, but not in a SSMA environment due to the presence of the interusier interference. In complex notation, the receiver performs an operation ecguivalont to correlating $r(t)$ of equation (2.8) with $\sqrt{\frac{2}{T}} a^{3}(t) \exp \left(-j \omega_{c} t\right)$. Defining $Y(t)$ to be 1 l', equivalent complex lowpass, output of the correlation process gives

$$
\begin{aligned}
Y^{\prime}(t) & =\sqrt{\frac{2}{T}} \int_{0}^{T} r(t) a^{2}(t) \exp \left(-j \omega_{c} t\right) d t \\
& =2 \frac{\sqrt{E_{s}}}{T} \int_{0}^{T}\left\{\operatorname{Re}\left[\sum_{k=1}^{K} \xi^{k}\left(t-\tau^{k}\right) \exp \left[j\left(\omega_{c} t+\beta^{k}\right)\right]+\|(t)\right\} a^{2}(1)(\cdot \times p)\left(-j \alpha^{\prime}, l\right) d t\right.
\end{aligned}
$$




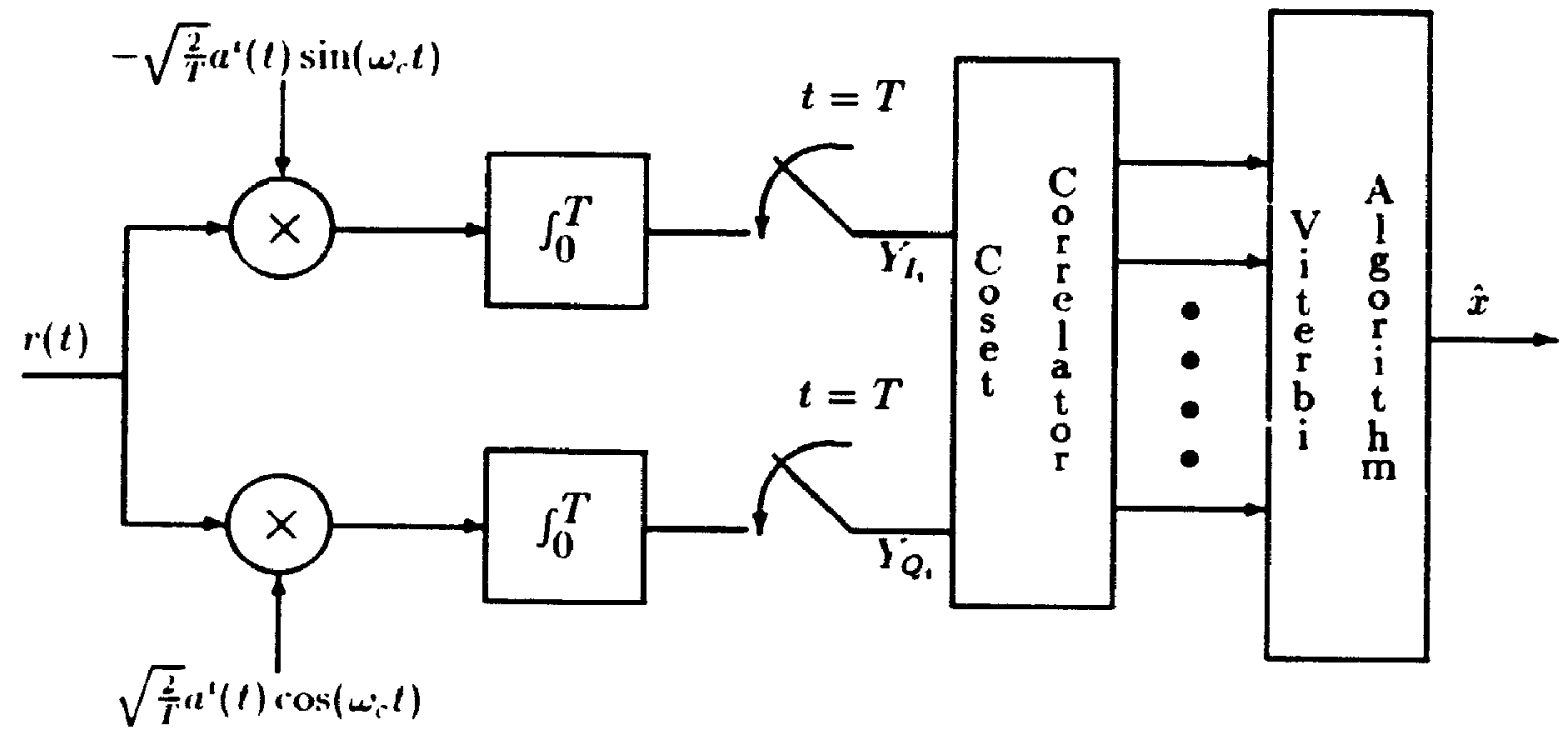

Figure 2.8: Receiver model 
Assuming without loss of generality that $\tau^{\prime}=0$ and $\beta^{k}=0$, the sampled complex input to the coset correlator at some arbitrary baud interval $p$ becomes

$$
Y_{p}^{i}=\sqrt{E_{0}}\left\{x_{p}^{i}+\frac{1}{T} \sum_{\substack{k=1 \\ k \neq i}}^{K}\left[x_{p-1}^{k} R_{k, 4}\left(\tau_{k}\right)+x_{p}^{k} \dot{R}_{k, 1}\left(\tau^{k}\right)\right] \exp \left(j \beta^{k}\right)\right\}+\eta_{p}^{i}
$$

in which $\eta_{p}^{i}$ is a complex sample of AWGN with variance $N_{0}$. The result of equation (2.13) is valid if one assumes that $\omega_{c} \gg T^{-1}$, in which case the higher order frequency terms are negligible. The even and odd continuous-time partial cross. correlation functions $R_{k, i}\left(\tau^{k}\right)$ and $\hat{R}_{k, i}\left(\tau^{k}\right)$ are defined by $[12][15]$

$$
\begin{aligned}
R_{k, \mathrm{i}}\left(\tau^{k}\right) & =\int_{0}^{\tau^{k}} a^{k}\left(t-\tau^{k}\right) a^{i}(t) d t \\
& =C_{k, i}\left(l-N_{c}\right) \hat{R}_{\psi}\left(\tau^{k}-l T_{c}\right)+C_{k, \mathrm{~s}}\left(l+1-N_{c}\right) R_{\psi}\left(\tau^{k}-l T_{c}\right) \\
\hat{R}_{k, i}\left(\tau^{k}\right) & =\int_{\tau^{k}}^{T} a^{k}\left(t-\tau^{k}\right) a^{1}(t) d t \\
& =C_{k, i}(l) \hat{R}_{\psi}\left(\tau^{k}-l T_{c}\right)+C_{k, i}(l+1) R_{\psi}\left(\tau^{k}-l T_{c}\right)
\end{aligned}
$$

in which the partial autocorrelation functions of the chip waveform are defined by $R_{\psi}(s)$ and $\hat{R}_{\psi}(s)[15]$

$$
\begin{aligned}
& R_{\psi}(s)=\int_{0}^{s} \psi(t) \psi\left(t+T_{r}-s\right) d t \\
& \hat{R}_{\psi}(s)=\int_{s}^{T_{c}} \psi(t) \psi(t-s) d t
\end{aligned}
$$

and the aperiodic cross-correlation function $C_{k, 1}(\cdot)$ is defincel by[15]

$$
C_{k, 1}(l)= \begin{cases}\sum_{j=0}^{N_{r}-1-l} a_{j}^{(k)} a_{j+l}^{(1)} & 0 \leq l \leq N_{c}-1 \\ \sum_{j=0}^{N_{r-1+1}} a_{j-1}^{(k)} a_{j}^{(1)} & 1-N_{r} \leq l<0 \\ 0 & |l| \geq N .\end{cases}
$$


For the rectangular chip waveforms employed in this study equations (2.14) and (2.15) take on the form

$$
\begin{aligned}
& R_{k, i}\left(\tau^{k}\right)=C_{k, i}\left(l-N_{c}\right) T_{c}+\left(\tau^{k}-l T_{c}\right)\left[C_{k, i}\left(l+1-N_{c}\right)-C_{k, i}\left(l-N_{c}\right)\right] \\
& \dot{R}_{k, i}\left(\tau^{k}\right)=C_{k, i}(l) T_{c}+\left(\tau^{k}-l T_{c}\right)\left[C_{k, i}(l+1)-C_{k, i}(l)\right]
\end{aligned}
$$

The coset correlator correlates the complex signal $Y_{p}^{i}$ with each of the possible signal points of the partitioned signal constellation (as was explained in Section 2.1.3). Recapitulating, this can be accomplished by correlating $Y_{p}^{i}$ with only one signal point from each antipodal coset. since the value of the correlation for any two signal points in the same antipodal coset, will have the same magnitudes but opposite polarities. Thus the number of computations can be reduced by half.

The first term on the left hand side of equation (2.13) is the transmitted symbol in the $\boldsymbol{p}^{\text {th }}$ baud interval. whereas the second term under the summation is the interuser interference (ILI) at the output of the correlators. Define the IUI seen by the $i^{\text {th }}$ user in the $p^{\text {th }}$ baud interval as

$$
\begin{aligned}
Z_{p}^{\prime} & =\sqrt{E_{s}}\left\{\frac{1}{T} \sum_{\substack{k=1 \\
k \neq i}}^{K}\left[x_{p-1}^{k} R_{k .1}\left(\tau^{k}\right)+x_{p}^{k} \dot{R}_{k .1}\left(\tau^{k}\right)\right] \exp \left(j \beta^{k}\right)\right\} \\
& =\sqrt{E_{s}}\left(j_{p}^{k} \exp \left(j \beta^{k}\right)\right.
\end{aligned}
$$

in which

$$
G_{p}^{\prime}=\frac{1}{T} \sum_{\substack{k=1 \\ k \neq 1}}^{K}\left[x_{p-1}^{k} R_{k, 1}\left(\tau^{k}\right)+x_{p}^{k} \hat{R}_{k, 3}\left(\tau^{k}\right)\right]
$$

is the amplitude and phase normalized IUI of the $i^{\text {th }}$ user during the $p^{\text {th }}$ baud. Combining equations (2.13), and (2.21) one obtains

$$
Y_{p}^{\prime}=I_{p}^{\prime}+Z_{p}^{s}+\eta_{p}^{\prime}
$$

in which $X_{p}^{-k}=\sqrt{E_{s}} x_{p}^{k}$. In the above and subsequent analysis. lower case has been 
employed to denote symbols or random variables that have been normalized with respect to $\sqrt{E_{s}}$, whereas upper case has been reserved for symbols or random variables that implicitly contain the square root of the symbol energy $E_{2}$.

The above analysis applies to trellis coded SSMA and convolutionally coderl SSMA systems in which the PN sequence spans one coded symbol. For the case in which a convolutionally coded system employs PN sequences that span n coded symbols, (or alternatively each coded symbol spans a fraction $\frac{1}{n}$ of the PN sequenc(e). it is necessary to modify equations (2.18) (2.19) and (2.20) appropriately in order to account for partial cross-correlation between subsequences of the: PN srquence. Following the notation of [20], define the $r^{\text {th }}$ subsequence of $a^{2}(t)$ as

$$
a_{(r)}^{2}=\left(a_{r L}^{2}, a_{r L+1}^{2}, \ldots a_{(r+1) L-1}^{1}\right)
$$

in which $\mathrm{L}$ is the length of the subsequences. Generalizing equation (2.18) to includv. subsequences gives[20]

$$
C[\mathbf{a} \cdot \mathbf{b}](l)= \begin{cases}\sum_{j=0}^{L-1-l} a_{j} b_{j+l} & 0 \leq l \leq l-1 \\ \sum_{j=0}^{L-1+l} a_{j-l} b, & 1-l \leq l<0 \\ 0 & |l| \geq l .\end{cases}
$$

in which $\mathbf{a}=\left(a_{0}, a_{1}, \ldots, a_{L}\right)$ and $\mathrm{b}=\left(b_{0}, b_{1}, \ldots \dot{\delta}_{L}\right)$. 'This allows onc (o) rewrit.' equations (2.19) and (2.20) as

$$
\begin{aligned}
& R\left[\mathbf{a}_{(r)}^{k}, \mathbf{a}_{(s)}^{\prime}\right]\left(\tau^{k}\right)=C\left[\mathbf{a}_{(r-1)}^{k} \cdot \mathbf{a}_{(s)}^{2}\right](l-L) T_{c}+\left(\tau^{k}-l T_{r}\right) \cdot \\
& {\left[C\left[\mathbf{a}_{(r-1)}^{2}, \mathbf{a}_{(s)}^{2}\right](l+1-L)-C \cdot \mathbf{a}_{(r-1)}^{2}, \mathbf{a}_{(s)}^{1}\right)(l-l, l]} \\
& \hat{R}\left[\mathbf{a}_{(r)}^{k}, \mathbf{a}_{(s)}^{\prime}\right]\left(\tau^{k}\right)=C:\left[\mathbf{a}_{(r)}^{k}, \mathbf{a}_{(s)}^{\prime}\right](l) T_{r}+\left(\tau^{k}-l T_{r}\right) . \\
& \left.\left[C\left[\mathbf{a}_{(r)}^{2}, \mathbf{a}_{(s)}^{z}\right](l+1)-C:\left[\mathbf{a}_{(r)}^{\prime}, \mathbf{a}_{(s)}^{(}\right)\right](l)\right]
\end{aligned}
$$


In urder to evaluate $R_{k, 3}(\tau)$ and $\hat{R}_{k, i}(\tau)$ of equations (2.19) and (2.20) to be used in equation (2.22), one can average equations (2.26) and (2.27) over all possible subsequences. For a PN sequence with period $N_{c}$ and a subsequence of length $L$, define the greatest common divisor of $M$ and $N_{c}$ as $G_{D}$. Then it can easily be shown that there are $q_{D}=N_{c} / G_{D}$ unique possible subsequences. Thus there are $q_{D}^{2}$ possible combinations of subsequences that one must average equations (2.26) and (2.27) over. It will be seen in Chapter 5 , that except when $N_{c}$ and $L$ are carefully chosen, that the number of computations involved in averaging over equations (2.26) and (2.27) is prohibitively large. 


\section{Chapter 3}

\section{Transfer Function Bounds}

The performance of trellis coded or convolutionally coded SSMA can be evaluated using transfer function bound techniques that have been developed for evaluating the performance of convolutional codes in AWGN[92] and have recently been applied to evaluating the performance of trellis codes in AWGN[87][88][89][90]. Since the transfer function bounds of convolutional codes can be formulated as a special case. of the generalized transfer function bound technique required for trellis codes, the following discussion will initially concentrate on transfer function bounds for trellis codes.

Viterbis initial derivation of the transfer function bound terhnique was aimurd at evaluating the performance of convolutional codes in an AW(iN channel. The trellis diagrams of the codes that were initially evaluated possessed symmetry comditions that allowed the performance to be evaluated by comparing all possible arror paths to the all zeros path. In general such conditions may not exist and an more. generalized transfer function bound technique was developed by Omura and Simon which accounted for all possible error paths when compared to all possible corrort paths[90]. This technique has been applied to evaluating the performance of 1 ral lis codes in AWGX by Dissalar and Simon[88][114] and also by Biglieri[x9]. The. generalized technique involves calculating the pairwise arrers between the correct 
and error sequences (by employing the Chernoff bound) and then summing river all possible patterns of correct and error sequences by using the union bound.

\subsection{General Derivation}

For the rate $\frac{n}{n+1}$ trellis codes that will be considered in this study, the trellis encoder selects one of $2^{n+1}$ signal points from the given constellation, for every $n$ input bits from the data source. Define the input bit sequence to the trellis encoder, at the $p^{\text {th }}$ bit interval to be

$$
u_{p}=\left(u_{p}, u_{p-1}, \cdots, u_{p-n+1}\right)
$$

The above and subsequent notation is based on that employed by Divsalar and Simon[114]. Corresponding to the sequence of input bits $u_{p}$, the trellis encoder will out put a symbol $x_{p}$ that is a nonlinear function $f(\cdot)$ of both $u_{p}$ and the state of the encoder $\delta_{p}$, i.e.

$$
\mathbf{x}=f\left(\delta_{p}, \mathbf{u}_{p}\right)
$$

Similarly, the next state of the encoder is a nonlinear function $g(\cdot)$ of the present state $d_{p}$ and the input vector $u_{p}$

$$
\delta_{p+1}=g\left(\delta_{p}, \mathbf{u}_{\mathbf{p}}\right)
$$

In the most gencral formulation of the transfer function bound, in which all possible correct seyuences must be compared to all possible incorrect sequences, it is useful to e'mploy' the concept of 'superstates' and 'super input signals'[89][90], defined as

$$
\begin{aligned}
& s_{p}=\left(\delta_{p}, \dot{\delta}_{p}\right) \\
& \zeta_{p}=\left(\mathbf{u}_{p} \cdot \dot{\mathbf{u}}_{p}\right)
\end{aligned}
$$

in whith $\varsigma_{p}$ and $u_{p}$ are the corrert state and input. whereas $\dot{\delta}_{p}$ and $\dot{u}_{p}$ are the incorrect state and input. 
From the definition of the complex baseband symbol output of the trellis or convolutional encoder of equation (2.1) define a complex baseband symbol channel input sequence of length $N$ by

$$
X=\left(X_{0}, X_{1}, \cdots, X_{N-1}\right)
$$

in which $\boldsymbol{X}_{\boldsymbol{p}}$ is the complex baseband symbol transmitted in the $p^{\text {th }}$ baud interval (the superscript $\boldsymbol{k}$ has been dropped without loss of generality for the present discussion). Corresponding to the channel input sequence $\mathbf{X}$. define a complex channel output symbol sequence (see equation (2.13))

$$
Y=\left(Y_{0}, Y_{1}, \cdots, Y_{N-1}\right)
$$

and a complex sequence of interuser interference as (see equation (2.21))

$$
\mathrm{Z}=\left(Z_{0}, Z_{1}, \cdots, Z_{N-1}\right)
$$

From equation (2.23), the complex channel output vector is

$$
\mathbf{Y}=\mathbf{X}+\mathbf{Z}+\boldsymbol{\eta}
$$

Using this notation. a general expression for the probability of bit crror can be shown to be[89][90]

$$
P_{b} \leq\left.\frac{1}{n} \frac{\partial T(I)}{\partial I}\right|_{I=1}
$$

in which $T(I)$ is the transfer function bound of the desires code. a veraged over the. interuser interference random vector $Z$ of equation (2.21), as denotud by theoverthar. In general, $T(I)$ can be expressed as

$$
T(D . I)=\sum_{N=1}^{\infty} \sum_{S\left(0 . N^{*}\right)} P\left(S_{0}\right) I^{d(\mathrm{~S}, \mathrm{U})} q\left(\mathrm{U}, I^{\prime}(\mathrm{X} \rightarrow \dot{\mathrm{X}})\right.
$$


in which $\mathbf{S}$ is a sequence of super states and $\mathbf{U}$ is a sequence of 'super input signals' defined respectively as

$$
\mathbf{S}=\left(S_{0}, S_{1}, \cdots, S_{N-1}\right)
$$

and

$$
\mathbf{U}=\left(U_{0}, U_{1}, \cdots, U_{N-1}\right)
$$

Furthermore in equation $(3.10) S(0, N)$ is a pair of correct and incorrect sequences, that diverge at time 0 , and remerge at time $N$, and $P\left(S_{0}\right)$ is the probability that the sequence started in state $S_{0}$. The indeterminate variable $I$ is employed as a base for the distortion measure $d(\mathbf{S}, \mathbf{U})$. In the case that will be studied the distortion is the Ilamming weigit of $U$, and as such can be expressed as $d(U)$. The term $q(U)$ denotes the probability of super input $U\left(\frac{1}{2^{n x}}\right.$ for the cudes under consideration ), and finally $P(\mathrm{X} \rightarrow \dot{\mathrm{X}})$ is the probability of a pairwise error event in which the incorrect vector $\dot{\mathbf{X}}$ is decoded in place of the correct vector $\mathbf{X}$. Frequently $\boldsymbol{P}(\mathbf{X} \rightarrow \dot{\mathbf{X}})$ can be replaced by the Bhattacharyya bound $D_{\lambda}(S, U)[88][90]$ however, for the present analysis the Bhattacharyya bound is not necessarily tight. In order to obtain the tightest bound un $P(\mathbf{X} \rightarrow \tilde{\mathbf{X}})$ it is necessary to optimize the more general Chernoff bound.

In order to a verage $T(I)$ over the random vector $Z$. one must possess knowladge of the probability density function of $Z$. For a SSMA system the probability density function of $Z$ is in genera! unknown. however from the definition of $Z_{p}^{a}$ of rquation (2.21), it is possible to calculate the moments of $Z$ up to any arbitrary order. From these moments an estimate of the probability density function can be detcrmined to any desired acurracy. The accuracy of the approximate density is a function of the number of moments employed in the evaluation. One such algorithm that has been employed in previous studies, and also in this study, is the method of muments $[\because \boldsymbol{y}][\because \cdot 2][\because 23]$. By enploring this algorithm one can account for the interuser inter irence due to other users in the SSMA system and thus evaluate the Chernoff bound for any pairwise cror. Conditioning on the random vector $Z$. and subseyuently taking the expectation over $\mathbf{Z}$ in order to obtain the average probability of 
a pairwise error. gives

$$
P_{E}=P(\mathbf{X} \rightarrow \overline{\mathbf{X}})=\mathbf{E}[P(\mathbf{X} \rightarrow \dot{\mathbf{X}} \mid \mathbf{Z})]
$$

If it is assumed that the interuser interference is independent from one symbol to the next, then employing the Chernoff bound (see section 4.2) allows one to upper boumd $P_{E}$ of equation (3.13) as a product of pairwise errors over individual symbols. 'The' method of moments can subsequently be employed to evaluate the pairwise error over a single symbol period as a weighted summation. The resulting expression for $P_{E}$ takes on the form

$$
P_{E} \leq \prod_{p=1}^{v} \sum_{j=0}^{N_{m}}\left|V_{p} P\left(X_{p} \rightarrow \dot{X}_{p} \mid Z_{r}\right)\right|_{Z_{r}=i_{p}}
$$

in which $I_{p}$, and $\zeta_{p,}$ are the $j^{\text {th }}$ weight and node (i.e. the abscissa at which Ifw. weighted function is to be evaluated), respectively in the $p^{\text {th }}$ symbol period. aal. c ' - ied from the moments of the interuser interference. and $N_{m}$ is the number of moments utilized to calculate the weights and nodes. The details of the calculation of the moments are provided in Appendix $A$. Using the definition of $F_{E}$ in erpuation (3.14) and the assumption of symbol independence allows one to express $T(I)$ as

$$
T(I)=\sum_{N=1}^{\infty} \sum_{s(0, N)} P\left(S_{0}\right) \prod_{p=0}^{N-1} I^{d\left(U_{p}\right)} q\left(U_{p}\right) \mathrm{E}\left[P\left(X_{p} \rightarrow \bar{X}_{r} \mid \zeta_{p}\right)\right]
$$

In the subsequent analysis the overbar shall be dropped without any less of generalit:

The a.bove expression for $T(I)$ can be evaluated in a systematic way l,y diffinint a matrix $T(I)$ with dimension $m^{2} \times m^{2}$ in which $m$ is the number of states in the. trellis code. In this matrix there is a row and column corresponding to each possilite. superstate (i.e. , each possible pair of correct and incorrect state pairs). The entry corresponding to the row of superstate $S_{p}$ and the column of superstate $S_{p}^{\prime}$ is given by

$$
[T(I)]_{\left(S_{p}, S_{p}^{\prime}\right)}=\left\{\begin{array}{l}
\sum_{u_{p}, \bar{u}_{p} \in U^{\frac{1}{2^{n}}} I^{d\left(U_{p}\right)} P\left(X_{p} \rightarrow \bar{X}_{p}\right)} \\
0 \text { if the transition } s_{p}^{*} \rightarrow S_{p}^{\prime} \text { not allowerl: }
\end{array}\right.
$$


U'sing the terminology of Biglieri[89] define a superstate as being 'good' if $\delta_{p}=\tilde{\delta}_{p}$, otherwise define the superstate as being 'bad'. Employing the subscripts ' $G$ ' and 'B' to denote good and bad respectively, one can partition the matrix $[T(I)]$ in a generic form as

$$
\mathbf{T}(I)=\left[\begin{array}{ccc}
\mathbf{T}_{G G}(I) & \vdots & \mathbf{T}_{G B}(I) \\
\ldots \ldots & \ldots \ldots . \\
\mathbf{T}_{B G}(I) & \vdots & \mathbf{T}_{B B}(I)
\end{array}\right]
$$

1.1 which the $m \times m$ submatrix $\mathbf{T}_{G G}(I)$ accounts for transitions between good superstates, $\mathbf{T}_{G B}(l)$ is an $m \times\left(m^{2}-m\right)$ submatrix accounting for transitions from good to bad superstates, $T_{H C}(I)$ is an $\left(m^{2}-m\right) \times m$ submatrix accounting for transitions from bad to good superstate;, and finally $\mathbf{T}_{B B}(I)$ is an $\left(m^{2}-m\right) \times\left(m^{2}-m\right)$ submaI rix accounting for transitions from bad to bad superstates. Using the definitions of the partition of equation (3.17), the transfer function bound of equation (3.10) can be written as[8?]

$$
T(I)=\frac{1}{m}\left\{a(I)+b^{T}(I) \cdot\left[\mathbf{I}-\mathbf{T}_{B B}(I)\right]^{-1} \mathrm{c}(I)\right\}
$$

in which

$$
\begin{aligned}
a(I) & =\mathbf{1}^{T} \cdot \mathbf{T}_{G G}(I) \cdot \mathbf{1} \\
\mathbf{b}(I) & =\mathbf{1}^{T} \cdot \mathbf{T}_{G B}(I) \\
\mathbf{c}(I) & =\mathbf{T}_{B G}(I) \cdot \mathbf{1}
\end{aligned}
$$

and 1 is a column vertor whose entries are all ones. Furthermore in equation (3.18) $I$ is the identity matrix. Substituting the expression of equation (3.18) into equation (3.9) taking the derivative and evaluating at $I=1$ gives the foliowing expression for the probability of bit error of [89]

$$
\begin{aligned}
& P_{i}=\frac{1}{m \cdot n}\left\{a^{\prime}(1)+b^{\prime T}(1) \cdot\left[\mathbf{I}-\mathbf{T}_{B B}(1)\right]^{-1} \cdot \mathbf{c}(1)+\right. \\
& \mathbf{b}^{r}(1)\left[\mathbf{I}-\mathbf{T}_{B B}(1)\right]^{-1} \cdot \mathbf{c}^{\prime}(1)+ \\
& \left.\mathbf{b}^{T}(1)\left[\mathbf{I}-\mathbf{T}_{B B}(1)\right]^{-1} \cdot \mathbf{T}_{B B}^{\prime}(1)\left\{\mathbf{I}-\mathbf{T}_{B B}(1)\right]^{-1} \cdot \mathbf{c}(1)\right\}
\end{aligned}
$$


In equation (3.22) ' denotes the derivative with respect to $I$.

The above upper bound for probability of bit error in equation (3.22) can be evaluated numerically on a computer. The most tedious step in the evaluation involves compiling the matrix $\mathbf{T}(I)$ of equation (3.17) which must be constructerl in a term by term fashion by using the matrix element definition of equation (3.16). For even a modestly complex code the number of elements in $\mathbf{T}(I)$ can berom. cumbersomely large since there are $m^{2} \times m^{2}$ terms to be generated. Ilowever, for a given trellis code and signal constellation it is possible to tabulate the Euclidean distances between all possible pairs of transmitted correct and error symbols (i.e. $x_{k}$ and $\tilde{x}_{k}$ ) for a given node in the rellis. From this stage it is straight forwaril to develop an algorithm that gencrates $\mathbf{T}(I)$. It should be pointed out that if thr. trellis code under consideration possesses symmetry properties, that it is frequently possible to reduce $\mathbf{T}(I)$ to an $m \times m$ matrix. Even if this is not the cast $\mathbf{T}(I)$ is usually a sparse matrix which allows one to employ numerically afficient sparse. matrix techniques in ihe evaluation of equation (3.22) [89].

\subsection{Transfer Function Bound Algorithm}

Given the signal constellation of the code under consideration and a systronatic de. scription of the enc der, an algorithm las been deveioperl to implement the general. ized transfer bound of equation (3.22). A prerequisite to evaluating erquation (3.2.2) is to generate the matrix $\mathbf{T}(I)$ as defined in equation (3.17).

For the trellis and convolutional codes under consideration in this study. thic. encoder can be represented in systematic form wit! feedback. Thus for any iufut $u_{n}$ it is possible to define the encoder logic of the given code in soft ware to produce thre coset output $\mu_{p}$ by the cuie (or the encoded symbols in the case of a convolutiontal code), as well as the next state $\delta_{p+1}$ in the trellis diagram. 'Thus as a first stop' towards generating equation (3.17) a table was created which contained for anch 
possible state $\delta_{p}$, the next state $\delta_{p+1}$, as well as the coset $\mu_{p}$ output. for all possible valuss of inputs $u_{p}$. For a rate $\frac{k}{n}$ code there will be $2^{k}$ possible inputs for each state of the code. resulting in $4 \times \mathrm{m} \lambda 2^{k}$ entries in the table (i.e. $\mathrm{m} \times 2^{k}$ entries of $\delta_{p}$, $\phi_{p+1}, \mathbf{u}_{p}$, and $\left.\mu_{p}\right)$.

The second step in the algorithm involves tabulating the intercoset Euclidean distance as well as the Hamming weight between the input symbois for all possible super state transitions. More explicitly, employing the data generated in step one above, for each permissible superstate transition $S_{p} \rightarrow S_{p+1}$ (for a superstate transition to be permissible. both of the transitions $\delta_{p} \rightarrow \delta_{p+1}$ and $\tilde{\delta}_{p} \rightarrow \tilde{\delta}_{p+1}$ must be possible according to the trellis diagram) the Euclidean distance between the ourput ignal points of the transitions $\delta_{p} \rightarrow \delta_{p+1}$ and $\tilde{\delta}_{p} \rightarrow \dot{\delta}_{p+1}$, as well as the Hamming weight between the inputs $\mathbf{u}_{p}$ and $\overline{\mathbf{u}}_{p}$ (i.e. $d\left(U_{p}\right)$ ), are tabulated. The Hamming weig', dt $d\left(U_{p}\right)$ defines the exponent of the variable $I$ of equation (3.16), whereas the ('her nolf bound $P\left(X_{p} \rightarrow \tilde{X}_{p}\right)$ can be evaluated for the intercoset distance of the superstate transition under consideration. The exact form of the Chernoff bound varies according to the channel condition: (i.e. AWGN or fading) and the metric chosen. l'he various forms of the Chernoff bound will be defined in subsequent chapters.

\subsubsection{Four State 4-PSK Code}

1 fur state. rate 1/2 1-J'SK trellis code will be used to illustrate the technique of alculating the generalized transfer function bound. From the signal constellation of the 1-15k rode (sere Figure 2.1), and the trellis state diagram for the code (ser? ligure 2.7 ) it is possilile to construct the generalized transfer function bound matrix: $\mathbf{T}(I)$ as is illustrated in Table 3.1. The entries of the matrix are calculated according forquation (3.16) in conjunction with the tre!lis tate diagram and the signal constellation. The ent ries of the matrix are as defined below

$$
\begin{aligned}
& a=1 \\
& b=I \cdot C\left(a_{i}\right)
\end{aligned}
$$




\begin{tabular}{|c|c|c|c|c|c|c|c|c|c|c|c|c|c|c|c|c|}
\hline & 00 & 11 & 22 & 33 & 01 & 02 & 03 & 10 & 12 & 13 & 20 & 21 & 23 & :30 & 31 & 32 \\
\hline 00 & $\mathbf{a}$ & $\mathbf{a}$ & 0 & 0 & b & 0 & 0 & b & 0 & 0 & 0 & 0 & 0 & 0 & 0 & 0 \\
\hline 11 & 0 & 0 & $\mathbf{a}$ & $\mathbf{a}$ & 0 & 0 & 0 & 0 & 0 & 0 & 0 & 0 & b) & 0 & (1) & h. \\
\hline 22 & $\mathbf{a}$ & $\mathbf{a}$ & 0 & 0 & b & 0 & 0 & b) & 0 & 0 & 0 & 0 & 0 & 0 & (I) & (1) \\
\hline 33 & 0 & 0 & $\mathbf{a}$ & $a$ & 0 & 0 & 0 & 0 & 0 & $\mathbf{v}$ & () & 0) & b, & (1) & () & l, \\
\hline 01 & 0 & 0 & 0 & 0 & 0 & c & $d$ & 0 & d & c. & 0 & 0 & 0 & () & () & 11 \\
\hline 02 & b & b & 0 & 0 & $\mathbf{a}$ & 0 & 0 & $a$ & 0 & 0 & () & 0 & () & () & () & () \\
\hline 03 & 0 & ) & 0 & 0 & 0 & $d$ & r & 0 & r & $d$ & 0 & 0 & () & 0 & () & 11 \\
\hline 10 & 0 & 0 & 0 & 0 & 0 & 0 & 0 & 0 & 0 & 0 & c & d & () & $d$ & c & 11 \\
\hline 12 & 0 & 0 & 0 & $\boldsymbol{U}$ & 0 & 0 & 0 & 0 & 0 & 0 & $d$ & c & () & $r$ & d & 11 \\
\hline 13 & 0 & 0 & $b$ & b & 0 & 0 & 0 & 0 & 0 & 0 & () & () & a & (I & 1) & $\mathbf{a}$ \\
\hline 20 & b & b & 0 & 0 & $\mathbf{a}$ & 0 & 0 & d & 0 & 0 & () & 0 & () & (1) & () & (I) \\
\hline 21 & 0 & 0 & 0 & 0 & 0 & $\mathrm{~d}$ & c & 0 & $c$ & $d$ & 0 & () & () & (1) & () & 11 \\
\hline 23 & 0 & 0 & 0 & 0 & 0 & c & d & 0 & d & - & 0 & (1) & (1) & 11 & 1) & 11 \\
\hline 30 & 0 & 0 & 0 & 0 & 0 & 0 & 0 & 0 & 0 & () & $d$ & c & (I) & c & $d$ & 11 \\
\hline 31 & 0 & 0 & $b$ & b & 0 & 0 & 0 & 0 & U & 0 & 1) & () & it & (1) & () & at \\
\hline 32 & 0 & 0 & 0 & () & 0 & () & 0 & () & () & 1, & r & 1 & (1) & d & , & (1) \\
\hline
\end{tabular}

Table 3.1: Superstate matrix for 4-state 4 lesh rate $1 / 21$ rollis arde. 


$$
\begin{aligned}
& c=C\left(\alpha_{1}\right) \\
& d=I \cdot C\left(\alpha_{1}\right)
\end{aligned}
$$

in which $I$ is an indeterminate variable employed to tag the bit errors, and

$$
C\left(\alpha_{i}\right)=P\left(x_{k} \rightarrow \tilde{x}_{k}\right)
$$

(i.e. the ('hernoff bound using energy normalized symbols), such that $\left|x_{k}-\tilde{x}_{k}\right|^{2}=a_{i}$. 'The values of $\alpha_{t}$ for the symmetrical 4-PSK constellation are tabulated in Table 2.1. It should be noted that the terms defined in equation (3.23) do not include the factor $\frac{1}{2^{\prime \prime}}$ of equation (3.16) which is the probability of a particular input being .uosen. assuming that all inputs are equiprobable.

The trellis state diagram and the signal constellation of this trellis code possess the necessary symmetry that will allow one to reduce the size of the generalized transfer function bo $\cdot 1$ matrix from a size of $16 \times 16$ to a size of $4 \times 4$. This reduction process can more easily be visualized as a two step reduction. Initially the $16 \times 16$ matrix will be recuced to an $8 \times 8$ matrix and subsequently to a $4 \times 4$ matrix. The first stage of the reduction can be achieved by noting that the following superstates are congruent (i.e. they can be combined into one superstate since they have the saume input and output transitions)

$$
\begin{aligned}
& 10 \Longleftrightarrow 22=\text { superstate } 0 \\
& 11 \Longleftrightarrow 33=\text { superstate } 1 \\
& 01 \Longleftrightarrow 10=\text { superstate } 2 \\
& 02 \Longleftrightarrow 20=\text { superstate } 3 \\
& 03 \Longleftrightarrow 30=\text { superstate } 4 \\
& 12 \Longleftrightarrow 21=\text { superstate } 5 \\
& 13 \Longleftrightarrow 31=\text { superstate } 6 \\
& 23 \Longleftrightarrow 32=\text { superstate } 7
\end{aligned}
$$


The resulting $8 \times 8$ transfer function bound matrix is illustrated in Table 3.2 in which the term elements are again as defined in equation (3.23). 'The labels for the combined row and column superstates were chosen to correspond to the righthand side of equation (3.25). A further reduction of the $8 \times 8$ matrix can be achievert by noting that the following superstates of Table 3.2 are congruent

$$
\begin{aligned}
& 0 \Longleftrightarrow 1 \text { relabeled as } 0 \\
& 2 \Longleftrightarrow 7 \text { relabeled as } 1 \\
& 3 \Longleftrightarrow 6 \text { relabeled as } 2 \\
& 1 \Longleftrightarrow 5 \text { relabeled as } 3
\end{aligned}
$$

The result is shown in Table 3.3 .

\subsubsection{Four State 8-PSK Code}

As a second example. consider the four state, rate $2 / 3$-PSh trellis code having lhe 8-PSK signal constellation of Figure 2.5. As in the previous section. the entries of the matrix $\mathbf{T}(I)$ are calculated according to equation (3.I(i) in conjunction with the' trellis state diagram and the signal constellation. The entries of the matrix arr an defined below

$$
\begin{aligned}
& a=I \cdot\left(\cdot\left(\alpha_{4}\right)\right. \\
& b=I(I+1) \cdot C^{\prime}\left(\alpha_{2}\right) \\
& c=I \cdot C^{\prime}\left(\alpha_{3}\right)+r^{\prime}\left(\alpha_{1}\right) \\
& d=I^{2} \cdot\left(\cdot\left(\alpha_{1}\right)+I \cdot\left(\cdot\left(\alpha_{3}\right)\right.\right. \\
& r=I \cdot\left(\cdot\left(\alpha_{1}\right)+I^{2} \cdot\left(\cdot\left(\alpha_{3}\right)\right.\right.
\end{aligned}
$$

in which $I$ is the indeterminate variable employed to tag the lif errers. and $r$ ' 6 e. 


\begin{tabular}{|c||c|c|c|c|c|c|c|c|}
\hline & 0 & 1 & 2 & 3 & 4 & 5 & 6 & 7 \\
\hline \hline 0 & $a$ & $a$ & $2 b$ & 0 & 0 & 0 & 0 & 0 \\
1 & $a$ & $a$ & 0 & 0 & 0 & 0 & 0 & $2 b$ \\
2 & 0 & 0 & 0 & $c$ & $d$ & $d$ & $c$ & 0 \\
3 & $b$ & $b$ & $2 a$ & 0 & 0 & 0 & 0 & 0 \\
\hline 4 & 0 & 0 & 0 & $d$ & $c$ & $c$ & $d$ & 0 \\
5 & 0 & 0 & 0 & $d$ & $c$ & $c$ & $d$ & 0 \\
6 & $b$ & $b$ & 0 & 0 & 0 & 0 & $c$ & $2 a$ \\
7 & 0 & 0 & 0 & $c$ & $d$ & $d$ & $c$ & 0 \\
\hline \hline
\end{tabular}

Table 3.2: $8 \times 8$ superstate matrix for 4-state 4-PSK rate $1 / 2$ trellis code

\begin{tabular}{|c||c|c|c|c|}
\hline & 0 & 1 & 2 & 3 \\
\hline \hline 0 & $2 \mathrm{a}$ & $2 \mathrm{~b}$ & 0 & 0 \\
1 & 0 & 0 & $2 \mathrm{c}$ & $2 d$ \\
2 & $2 \mathrm{~b}$ & $2 \mathrm{a}$ & 0 & 0 \\
3 & 0 & 0 & $2 d$ & $2 \mathrm{c}$ \\
\hline \hline
\end{tabular}

lalile 3.3: $1 \times 4$ superstate matrix for 4 -state 4 -PSK rate $1 / 2$ trellis cude 
is defined by equation (3.24) such that $\left|x_{k}-\dot{x}_{k}\right|^{2}=\alpha_{1}$. The values of $\alpha_{1}$ for the symmetrical 8-PSK constellation are tabulated in Table 2.1.

Again the trellis state diagram and the signal constellation of the trellis corle possess the necessary symmetry that will allow one to reduce the size of the generalized transfer function bound matrix from a size of $16 \times 16$ to a size of $4 \times 4$. The' congruent states in the reduction process are identical to those of equations (3.25) and (3.26) of the rate $\frac{1}{2}$ 4-state 4-PSK code. The corresponding $16 \times 16,8 \times \times$ and $4 \times 4$ matrices for the 8-PSK code are defined in tables 3.4. 3.5. and 3.6.

\subsubsection{Encoder Diagrams}

As was mentioned previously in section 3.2. the entries of the generalized transfer function bound matrix $\mathbf{T}(I)$ can be calculated from a knowledge of the signal con stellation being employed by the trellis code, as well as a knowledge of the logic of the encoder of the trellis code under consideration. Figures 3.1 to 3.6 define the encoders. in diagramatic form. of the codes that will be analymed in this study: namely, 4-PSK (2, 4 and 8 states). 8-PSK (2, 4, 8 and 16 states $)$ and 16-PSK (4, 8 and 16 states) trellis codes. These codes are standard trellis colls that hate. been analyzed for the AlVGN channel (see [6]. [88] and [102]). 'The ancorlers mill, $n$ inputs $\left(u_{0}, u_{1} \ldots u, v-1\right)$ to $n+1$ outputs $\left(\mu_{0}, \mu_{1}, \ldots \mu_{n}\right)$, which stedert a sperifis coset or signal constellation. The mapping of the outputs $\left(\mu_{1}, \mu_{1}, \ldots, \mu_{n}\right)$ itul, the. signal constellation are defined in Figures 2.4. 2.5 and 2.6 for the 1-PSK. S-PSK atul 16-PSK constellations respectively (ser also [i]]).

\subsubsection{Convolutional Codes}

The performance of convolutionally coded SSMA can bre revaluated using Ifre sarfu" algorithm described in Sertion 3.2. with some minor modifications. I lar majon lif ference between tha inplementation of the trellis coder SSMA system and the oul volutionally coded SSMA system is that the convolutional coder ontputs modulate. 


\begin{tabular}{|c|c|c|c|c|c|c|c|c|c|c|c|c|c|c|c|c|}
\hline & 00 & 11 & 22 & .33 & 01 & 02 & 03 & 10 & 12 & 13 & 20 & 21 & 23 & 30 & 31 & 32 \\
\hline 00 & $\mathbf{a}$ & $\mathbf{a}$ & 0 & 0 & $\mathbf{b}$ & $\mathbf{0}$ & 0 & $\mathbf{b}$ & 0 & 0 & 0 & 0 & 0 & 0 & 0 & 0 \\
\hline 11 & 0 & 0 & $\mathbf{a}$ & $\mathbf{a}$ & $\mathbf{0}$ & 0 & 0 & 0 & 0 & 0 & 0 & 0 & $\mathbf{b}$ & 0 & 0 & b \\
\hline 2.2 & $\mathbf{a}$ & $a$ & 0 & 0 & b & 0 & 0 & b & 0 & 0 & 0 & 0 & 0 & 0 & 0 & 0 \\
\hline 333 & 0 & 0 & $a$ & $\mathbf{a}$ & () & 0 & 0 & 0 & 0 & 0 & 0 & 0 & $\mathbf{b}$ & 0 & 0 & b \\
\hline 01 & 0 & 0 & 0 & 0 & 0 & $c$ & $d$ & 0 & $e$ & c & 0 & 0 & 0 & 0 & 0 & 0 \\
\hline (1)2 & b) & b & () & 0 & a & 0 & 0 & $\mathbf{a}$ & 0 & 0 & 0 & 0 & 0 & 0 & 0 & 0 \\
\hline 03 & 0 & 0 & 0 & 0 & 0 & $d$ & c & 0 & c & e & 0 & 0 & 0 & 0 & 0 & 0 \\
\hline 10 & 0 & 0 & 0 & 0 & 0 & 0 & 0 & $\mathbf{0}$ & 0 & 0 & $c$ & $e$ & 0 & $d$ & $r$ & 0 \\
\hline $1: 3$ & () & 0 & 0 & 0 & 0 & 0 & 0 & 0 & 0 & 0 & $e$ & $c$ & 0 & c & $\mathrm{d}$ & 0 \\
\hline 13 & 0 & 0 & b) & b & 0 & 0 & 0 & 0 & 0 & 0 & 0 & 0 & $\mathbf{a}$ & 0 & $\mathbf{0}$ & $\mathbf{a}$ \\
\hline 20 & b) & $b$ & 0 & 0 & $\mathbf{a}$ & 0 & $n$ & $\mathbf{a}$ & 0 & 0 & 0 & 0 & 0 & 0 & 0 & 0 \\
\hline 21 & 0 & 0 & 0 & 0 & 0 & e & c & 0 & $c$ & $d$ & 0 & 0 & 0 & 0 & 0 & 0 \\
\hline 23 & 0 & () & () & () & 0 & r & e & 0 & $d$ & c & 0 & 0 & 0 & 0 & 0 & 0 \\
\hline 30 & 0 & 0 & 0 & 0 & 0 & 0 & 0 & 0 & 0 & 0 & d & $c$ & 0 & c & $e$ & 0 \\
\hline 31 & () & 0 & b & $b$ & 0 & 0 & 0 & 0 & 0 & 0 & 0 & 0 & $\mathbf{a}$ & 0 & 0 & a \\
\hline $3: 2$ & 0 & 0 & 0 & 0 & 0 & 0 & 0 & 0 & 0 & U & $r$ & $d$ & 0 & e & $c$ & 0 \\
\hline
\end{tabular}

Tablo 3.1: Superstate matrix for 4-state 8-PSK rate $2 / 3$ trellis code 


\begin{tabular}{|c||c|c|c|c|c|c|c|c|}
\hline & 0 & 1 & 2 & 3 & 4 & 5 & 6 & 7 \\
\hline \hline 0 & $a$ & $a$ & $2 b$ & 0 & 0 & 0 & 0 & 0 \\
1 & $a$ & $a$ & 0 & 0 & 0 & 0 & 0 & $2 b$ \\
2 & 0 & 0 & 0 & $c$ & $d$ & $e$ & $c$ & 0 \\
3 & $b$ & $b$ & $2 a$ & 0 & 0 & 0 & 0 & 0 \\
\hline 4 & 0 & 0 & 0 & $d$ & $c$ & $c$ & $e$ & 0 \\
5 & 0 & 0 & 0 & $e$ & $c$ & $c$ & $d$ & 0 \\
6 & $b$ & $b$ & 0 & 0 & 0 & 0 & $c$ & $2 a$ \\
7 & 0 & 0 & 0 & $c$ & $c$ & $d$ & $c$ & 0 \\
\hline \hline
\end{tabular}

Table 3.5: $8 \times 8$ superstate natrix for 4 -state 6 -PSK rate $: / 3$ trellis rode

\begin{tabular}{|c||c|c|c|c|}
\hline & 0 & 1 & 2 & 3 \\
\hline \hline 0 & $2 \mathrm{a}$ & $2 \mathrm{~b}$ & 0 & 0 \\
$\mathrm{i}$ & 0 & 0 & $2 \mathrm{c}$ & $d+c$ \\
2 & $2 \mathrm{~b}$ & $2 \mathrm{a}$ & 0 & 0 \\
3 & 0 & 0 & $d+$, & $2 \mathrm{c}$ \\
\hline
\end{tabular}

Table 3.6: $4 \times 4$ superstate matrix for 4 -state $\times$-PSh sate $2 / 31$ rollis conto 


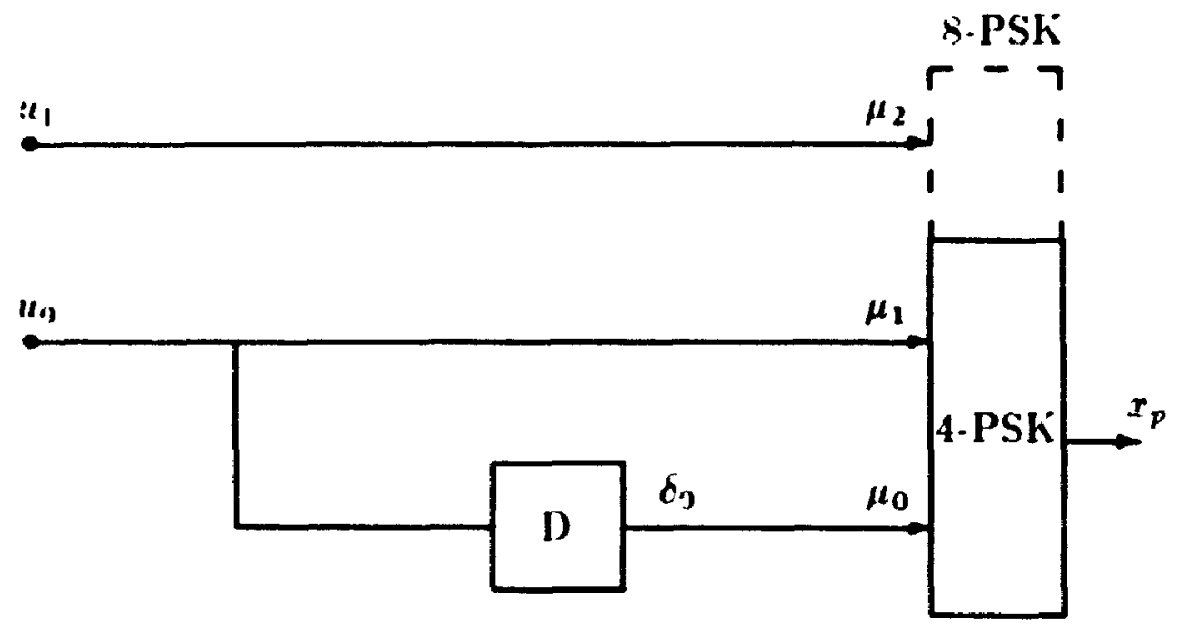

Figure 3.1: Sistenatic 2-stale rate $1 / 24-\mathrm{PSh}$ and 2 -state rate $2 / 3$ 8-PSK trellis encouders 


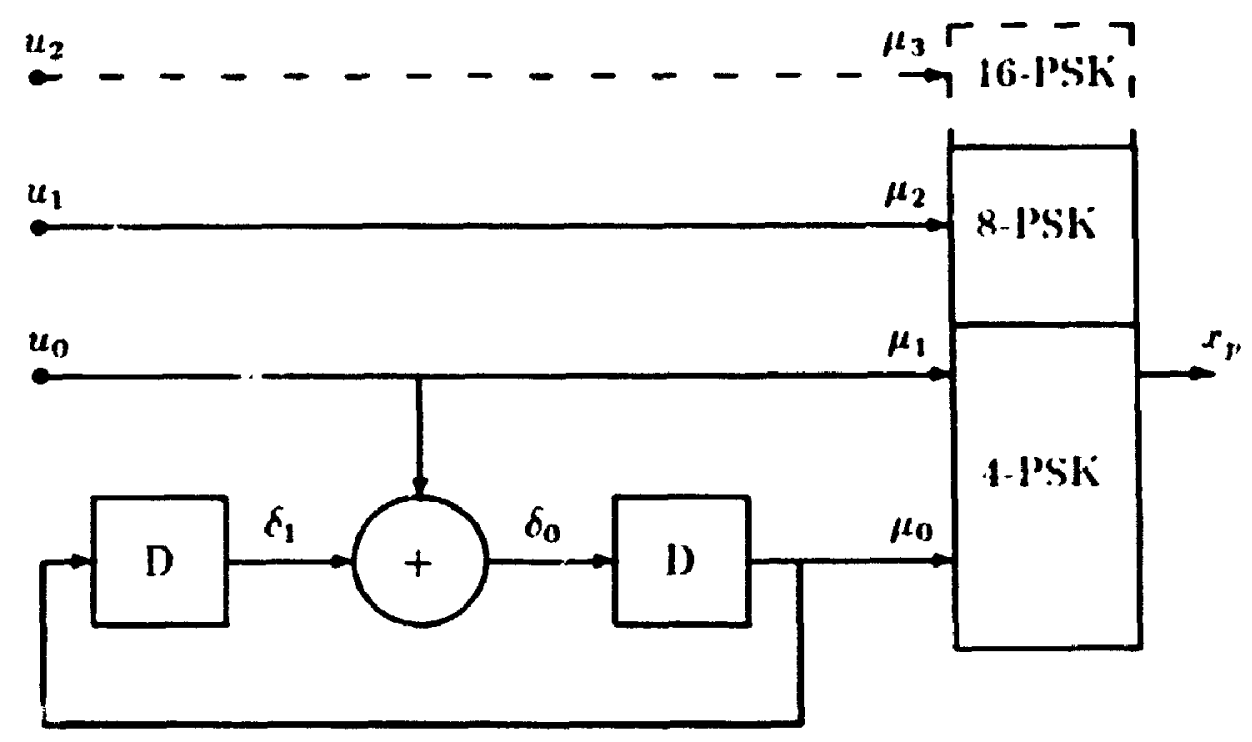

Figure 3.2: Sistematic 4-state rate 1/2 4-PSK . 4-stale rate $2 / 3 \times$. I'Sk and 1 statle rate $3 / 4$ 16-PSK t rellis cncoders 


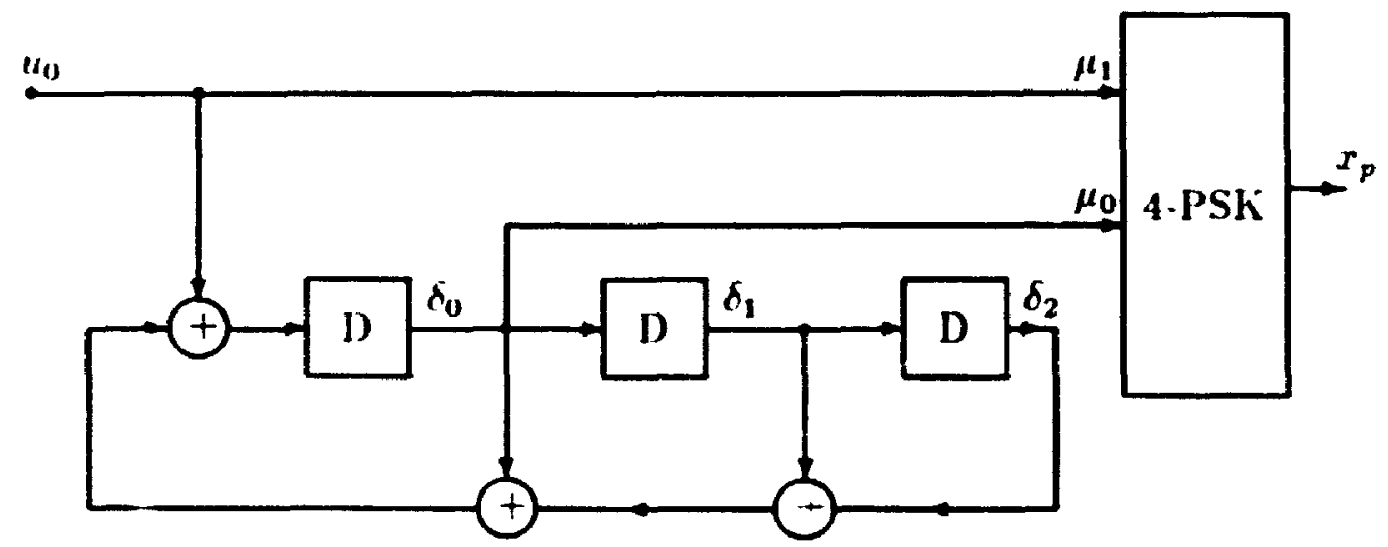

Figure 3.3: Sistematic 8-state rate 1/2 4-PSK trellis encoder 


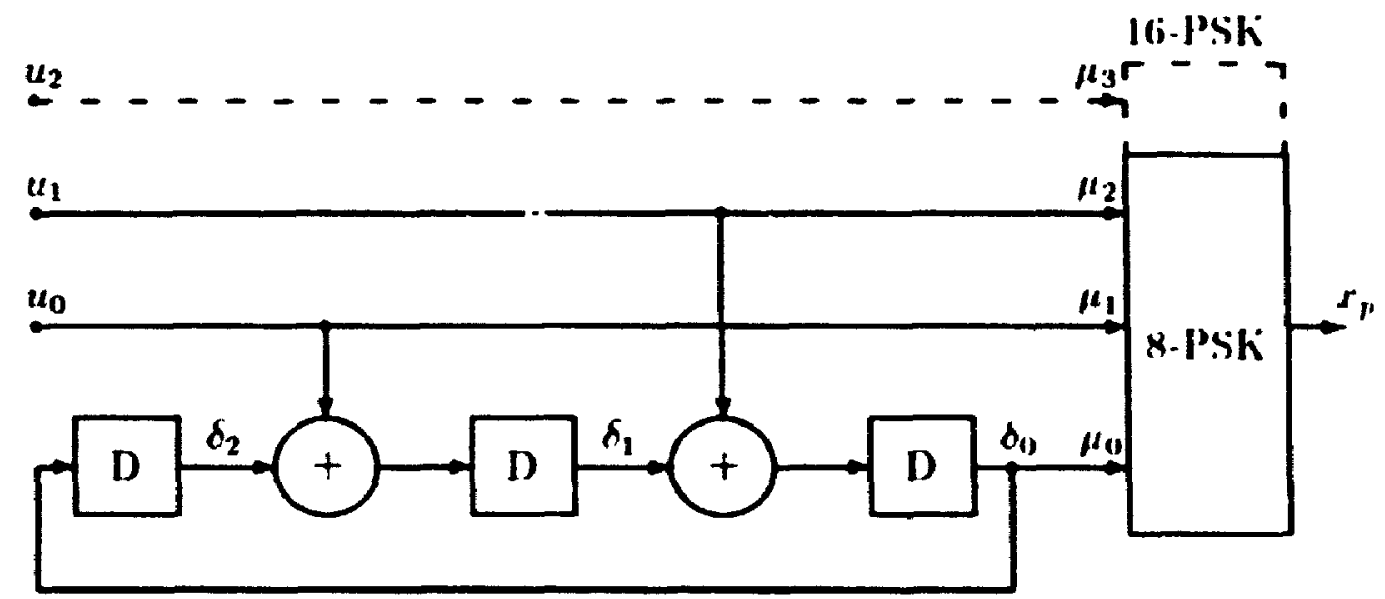

Figure 3.4: Systematic 8 -state rate $2 / 3$ 8 -PSK and $x$-state rate $3 / 416 \cdot 1$ - ih 1 rellis encoders 


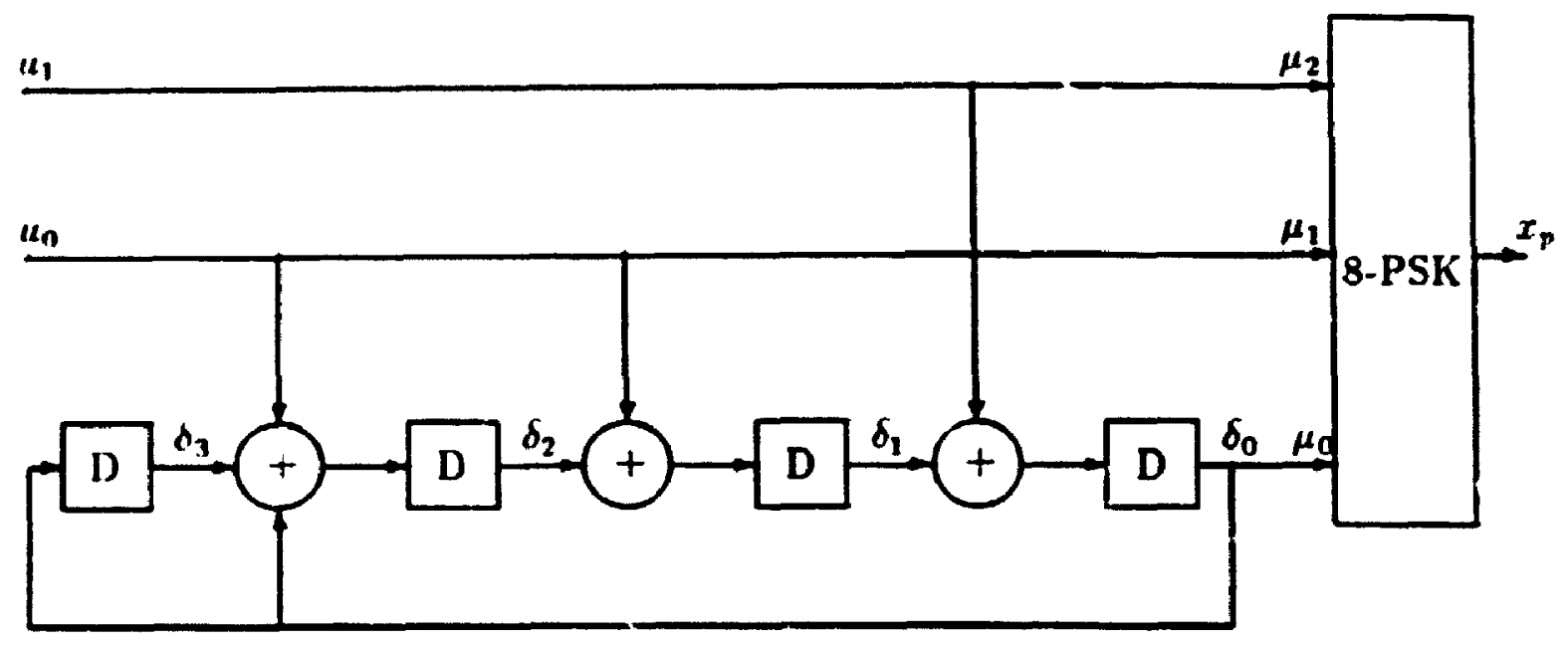

Figure 3.5: Systematic 16-state rate 2/3 8-PSK trellis enroder 


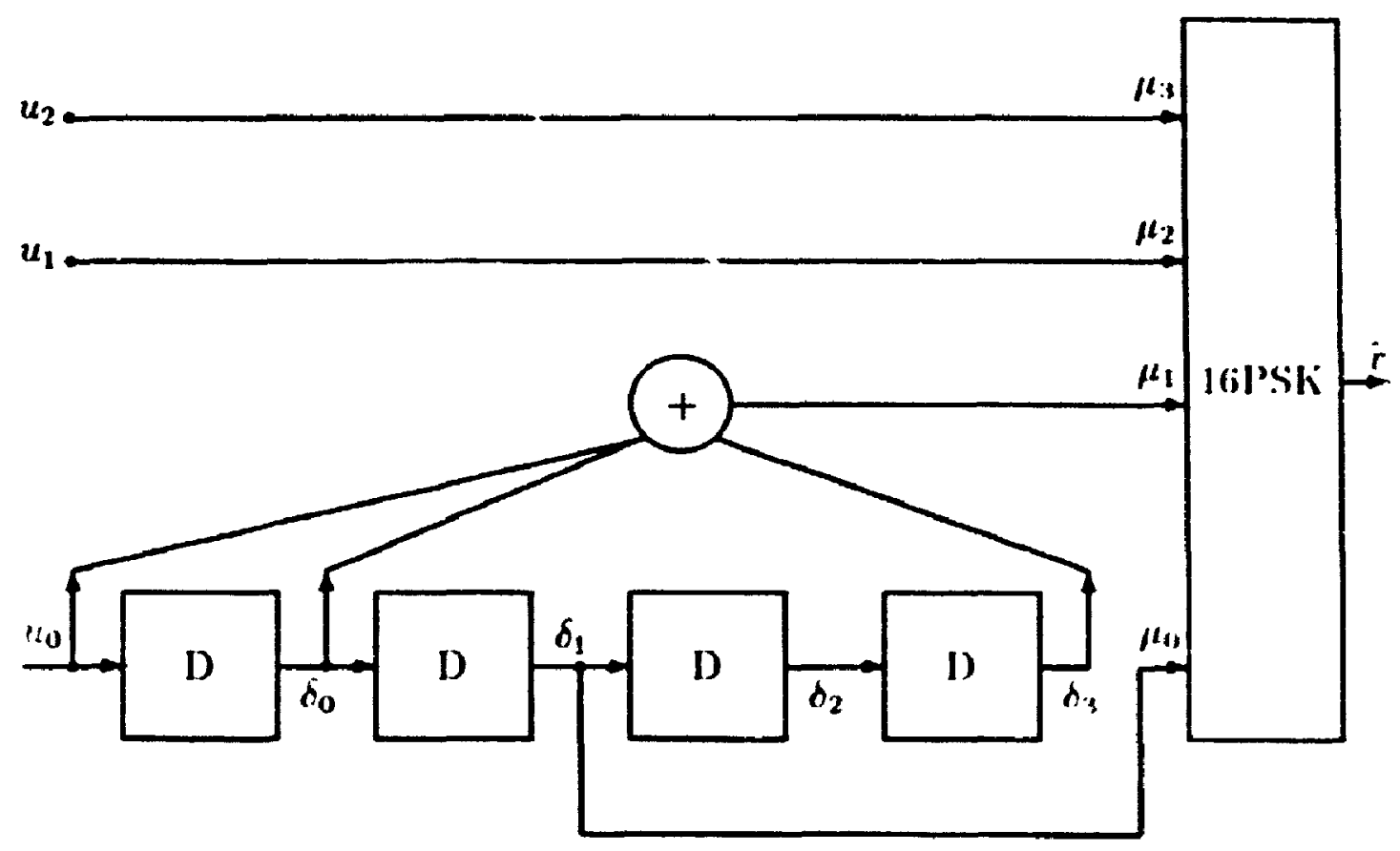

Figure 3.6: Encoder for the 16-PSK 16 state rate $3 / 4$ tr. ine orde 
the PX serquence directly, with the subsequent signal being transmitted in a BFSR signal format. Since the convolutional codes are to be compared to the trellis codes (I) the basis of equal throughput, in general, for a rate $\frac{k}{n}$ code, there will be $n$ coded output symbols for each $h$ information bits. with each coded symbol being reciuced in energy by a factor of $\frac{k}{n}$ with respect to the energy per bit. Thus the coset output during a transition from one superstate to another superstate will consist of $n$ coded symbols, the Ilamming woight of which is employed to evaluate the Chernoff bound pitirwise error. Moresperifically, since the coded symbols take on binary values $( \pm 1)$. the 'intercoset llamming woight' between $n$ correct coded symbols and $n$ incorrect coled symbols can le translated into an intercoset Euclidean distance since for a IBl'Sk signal constellation a llamming distance of 1 maps 10 a squared Euclidean distance of 1.0. For the scenario in which the interuser interference random variable. $\%$ is assumed to be independent from one coded symbol to the next. $Z$ will be averaged over each coded sumbol independently. The Hamming weight of $d\left(t_{p}\right)$ is emploved in an identical manner to that described for the trellis codes.

The convolutional codes that were considered in this study are rate $\frac{1}{2}, \frac{1}{8}, \frac{2}{3}$, and $\doteq$ colles. having 1. s and 16 states. The generator polynomials of these codes are tabulated in lable 3 [110]. Examples of the 4 -state rate $\frac{1}{2}$ encoder and the 4 -state rate.

\subsection{Minimum Distance Computation}

1ha matrix representation of the generalized transfer function bound derived in the previous section can also be utilized to evaluate the minimum squared Euclidean distance of the cole under consideration. Since one is only interested in the minimum listance. for the purposes of this discussion the channel in which the trellis code is operating can be considered to be the AlVGN channel. Under these assumptions, the ('hermoff hominl $I^{\prime}\left(X_{p} \rightarrow \dot{X}_{p}\right)$ of cquation (3.16) can be rewritten as a Bhattacharyya 


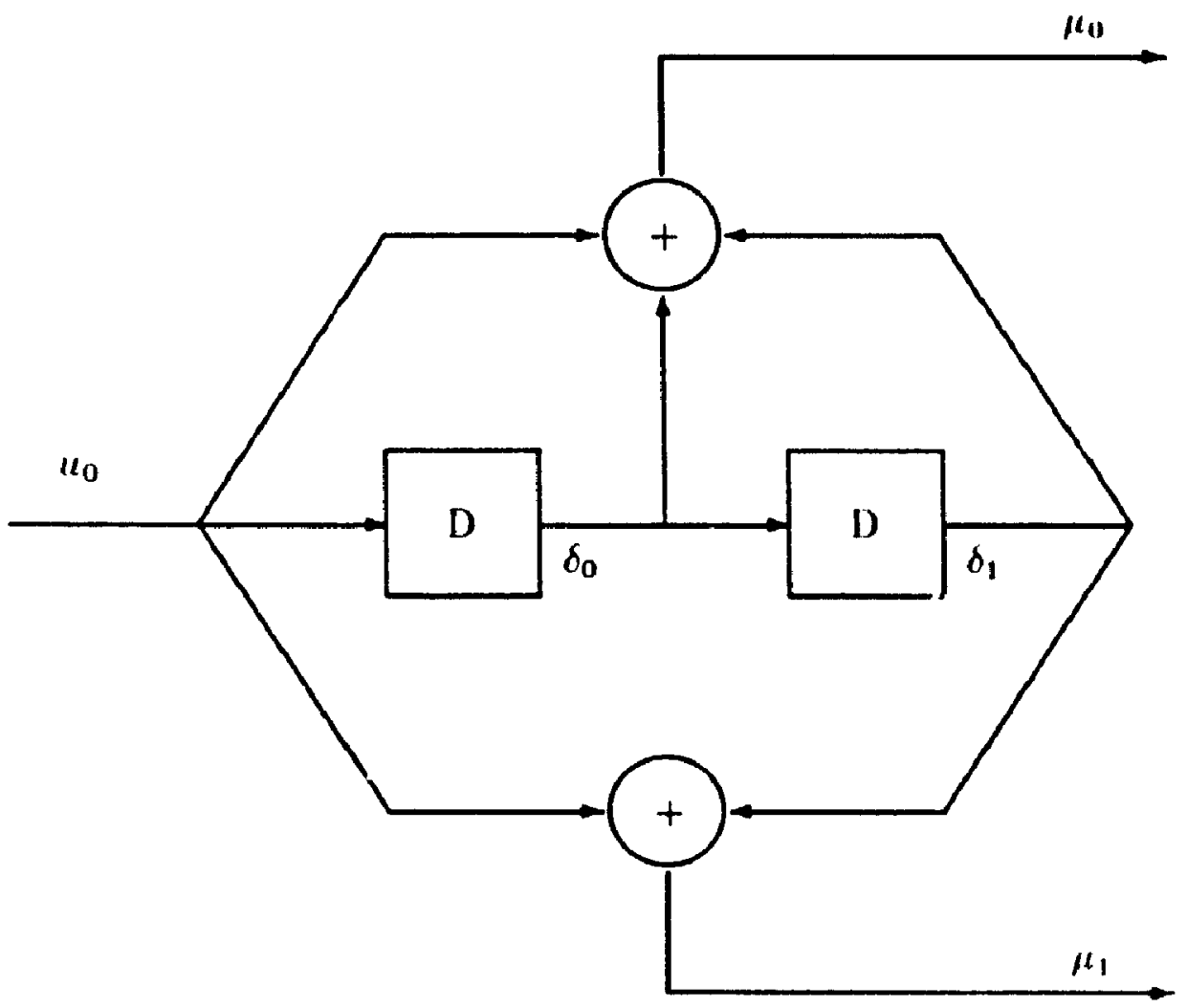

Figure 3.7: Encoder for the 4-state rate $1 / 2$ convolutional code 


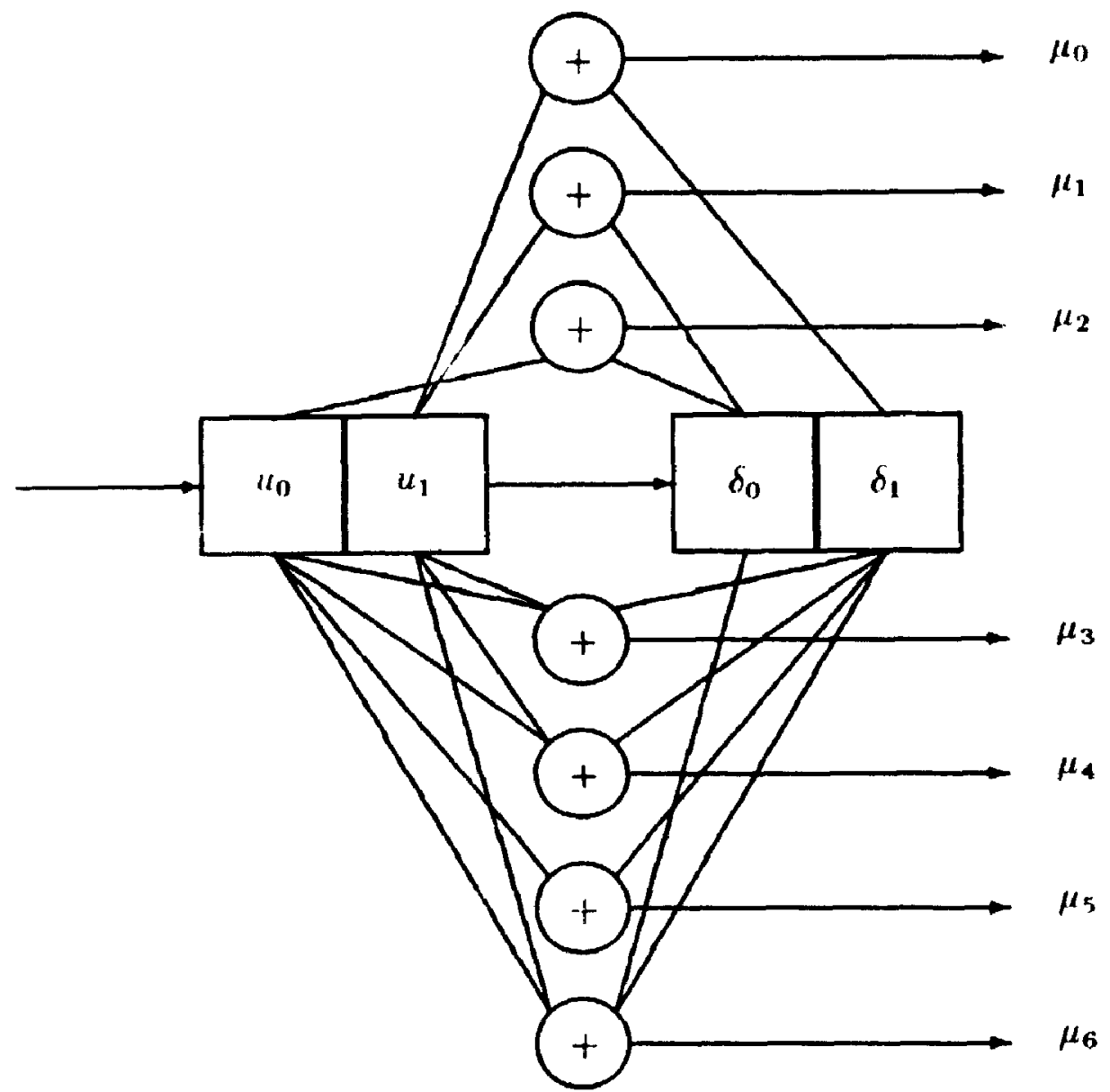

Figure 3.x: Encoder for the 4-state rate $2 / 7$ convolutional code 
bound $[88][89][92]$. resulting in a transfer function bound of the form

$$
\begin{aligned}
T(D) & =f_{1} D^{d_{m i n}^{2}}+f_{2} D^{d_{2}^{2}}+f_{3} D^{d_{1}^{2}}+\ldots \\
& =\sum_{i=1}^{\infty} f_{1} D^{d_{i}^{2}}
\end{aligned}
$$

in which $d_{1}=d_{m ! n}^{2}$ and

$$
D=\exp \left(-\frac{1}{8 \sigma^{2}}\right)
$$

is the Bhattacharyya parameter[88] $\left(\sigma^{2}\right.$ is the variance of the (ialssian noise $)$. Fur the purposes of this discussion, the terms $f_{1}, f_{2}, \ldots f_{1} \ldots$ of equalion (3.28) a.n ln. treated as constants. and $d_{m: n}<d_{2}<d_{3}<\ldots<d_{t}$ are the Euclidean distances of the respective paths. Furthermore. it follows that

$$
\begin{aligned}
T(e D) & =f_{1}(e D)^{a_{\text {nan }}^{2}}+f_{2}(c D)^{d_{2}^{2}}+f_{3}(e D)^{d_{1}^{2}}+\ldots \\
& =(e D)^{d_{\operatorname{man}}^{2}}\left(f_{1}+\sum_{i=2}^{\infty} f_{1}(e D)^{\left.d_{i}^{2}-d_{\operatorname{man}}^{2}\right)}\right.
\end{aligned}
$$

in which $e$ is the base of the natural logarithm. Factoring out $d_{m a n}^{2}$ from "alla tion (3.28) allows one to express the ratio of $T(e D)$ over $T(D)$ as

$$
\frac{T(e D)}{T(D)}=\exp \left(d_{m+n}^{2}\right) \cdot \frac{\left(f_{1}+\sum_{i=2}^{\infty} f_{1}(c D)^{i_{1}^{2}-t_{m+n}^{2}}\right)}{\left(f_{1}+\sum_{i=2}^{\infty} f_{1}(D)^{\left.i_{1}^{2}-t_{m+n}^{2}\right)}\right.}
$$

Taking the natural logarithm of equation (3.31) followed! by the limit as $D \rightarrow($ g,ivan

$$
\begin{aligned}
& \lim _{D \rightarrow 0} \ln \left[\frac{T(e D)}{T(D)}\right]=d_{m 2 n}^{2}+\lim _{D \rightarrow 0} \ln \left[\frac{\left(f_{1}+\sum_{i=2}^{\infty} f_{2}(c D)^{\left.d_{2}^{2}-d_{m(n)}^{2}\right)}\right.}{\left(f_{1}+\sum_{i=2}^{\infty} f_{2} D^{\left.d_{1}^{2}-d_{m a n}^{2}\right)}\right.}\right] \\
& =d_{m \geq n}^{2}+\ln \left[\frac{f_{1}}{f_{1}}\right] \\
& =d_{m: n}^{2}
\end{aligned}
$$


which is the desired result. It should be noted that in the construction of the matrix $T(D)$ (see equations (3.16)and (3.17)), that the entry for a given superstate row and column is modifed so that it only contains the term corresponding to the miniunum distance transition as opposed to the summation over all possible transitions. A final point is that the choice of the natural base in equation (3.30) is arbitrary: Divsalar[xy] chose a hase of 2, which makes the evaluation somewhat simpler nuImerically. The minimum distances of the trellis and convolutional codes considered in this study are listed in Table 3.8. The second last column gives the minimum Fuclidean distance for the trellis codes, whereas it gives the ninimum Hamming distance for thre convolutional codes. The last column provides the gain of the re-pertive codes wer the corresponding uncuded constellation (i.e. BPSK for rate $1 / \mathrm{n}$ coles, QPSK for rate 2 in rodes, etc. ). 


\begin{tabular}{||c|c|c||}
\hline \hline rate & no. states & generator polynomials in octal \\
\hline \hline $1 / 2$ & 1 & 57 \\
$1 / 2$ & 8 & 1517 \\
$1 / 2$ & 16 & 2335 \\
$1 / 8$ & 4 & 77555777 \\
$1 / 8$ & 8 & 1717131313151517 \\
$1 / 8$ & 16 & 3733252535332737 \\
$2 / 3$ & 4 & 170615 \\
$2 / 3$ & 16 & 277572 \\
$2 / 7$ & 4 & 05061215151317 \\
$2 / 7$ & 16 & 33557247255375 \\
$1 / 7$ & 4 & 7775555 \\
\hline \hline
\end{tabular}

Table 3.7: Generator polynomials of convolutional corjes 


\begin{tabular}{|c|c|c|c|c|}
\hline code & rate & $\begin{array}{c}\text { no. } \\
\text { states }\end{array}$ & $d_{m: n}$ & $\begin{array}{c}\text { gain over } \\
\text { uncoded system } \\
\text { [dB] }\end{array}$ \\
\hline 4-PSK trellis & $1 / 2$ & 2 & 8.0 & 3.0 \\
\hline 4-PSK trellis & $1 / 2$ & 4 & 10.0 & 3.98 \\
\hline 1-PSK trellis & $1 / 2$ & 8 & 12.0 & 4.77 \\
\hline 8-PSK trellis & $2 / 3$ & 2 & 2.586 & 1.12 \\
\hline R-PSh trellis & $2 / 3$ & 4 & 4.0 & 3.01 \\
\hline 8-PSh trellis & $2 / 3$ & $x$ & 4.586 & 3.6 \\
\hline Q.PSh trollis & $2 / 3$ & 16 & 5.172 & 4.1 \\
\hline 16-PSK trellis & $3 / 4$ & 4 & 1.324 & 3.54 \\
\hline 16-PSK trellis & $3 / 4$ & 8 & 1.476 & 4.01 \\
\hline 16-PSK trellis & $3 / 4$ & 16 & 1.629 & 4.4 \\
\hline convolutional & $1 / 2$ & 4 & 5 & 3.97 \\
\hline convolutional & $1 / 2$ & 8 & 6 & 4.77 \\
\hline convonutional & $1 / 2$ & 16 & 8 & 6.02 \\
\hline convolutiunal & $1 / 8$ & 4 & 21 & 4.19 \\
\hline convolutional & $1 / 8$ & 8 & 26 & 5.11 \\
\hline convolutional & $1 / 8$ & 16 & 32 & 6.02 \\
\hline convolutional & $2 / 3$ & 4 & 3 & 1.76 \\
\hline convolutional & $2 / 3$ & 16 & 5 & 5.22 \\
\hline convolutional & $2 / 7$ & 4 & 9 & 4.1 \\
\hline convolutional & $2 / 7$ & 16 & 14 & 6.02 \\
\hline
\end{tabular}

lable 3.s: Comparison of the minimum distances of the trellis and convolutional codes studied 


\section{Chapter 4}

\section{Performance Analysis in an AWGN Channel}

Chapter 3 outlined the manner in which the generaized transfer function bound an be employed to evaluate the desired performance of any trellis code (or comwolutional code), given the encoder logic and the signal constellation. For a given coule, the. probability of bit error perf ormance is determined by the chanmel characteristics. which defines the form of the pairwise error probability in expation (3.16). Fur thermore. the calculation of the transfer function bound involves summing over all possible pairwise error events using the union bound. In order to make lhe selutient tractable, the pairwise error 'vents are usually upper bounderl using a ( acrnoff type bound, although it is also of interest to determine the exact form of the pairwise er ror probability. Thus the key step is the calculation of the pairwise error probability which fro'n equation (3.16) is given by

$$
P_{E}=P\left(X_{p} \rightarrow \dot{X}_{p}\right)=\mathbf{E}\left[P\left(X_{p} \rightarrow \dot{X}_{p} \mid Z_{p}\right)\right]
$$

in which the error probability has been averaged over the interuser interforence random variable $Z_{p}$ of equation (2.21) 


\subsection{Exact Pairwise Error Probability}

From equation (2.23) one can define the received decision variable over an $\mathrm{N}$-symbol period as an $\mathbf{N}$-dimensional complex random vector dehned by

$$
\mathbf{Y}=\mathbf{X}+\boldsymbol{\eta}+\mathbf{Z}
$$

in which $Y, X$ and $Z$ are as defined in equations (3.5), (3.6) and (3.7). The vector $\eta$ is a vertor of samples of complex AWGN with variance $N_{0}$ defined as

$$
\eta=\left(\eta_{0}, \eta_{1}, \cdots, \eta_{N-1}\right)
$$

Define all error patcern vertor $D$ as

$$
\mathbf{D}=\mathbf{X}-\dot{\mathbf{x}}
$$

in which $\dot{\mathbf{X}}$ in the symlul ve tor detected in error when $\mathbf{X}$ was transmitted. For the maximum !ikelihood metric under consideration, such an error pattern will occur if

$$
|\mathbf{Y}-\dot{\mathbf{X}}|^{2} \leq|\mathbf{Y}-\mathbf{X}|^{2}
$$

lising equation $(1.2)$ in conjunction with equation (1.5) one obtains

$$
|\mathbf{D}+\eta+\mathbf{Z}|^{2} \leq|\eta+\mathbf{Z}|^{2}
$$

Ifter some algelora. this can be shown to be equivalent to

$$
\operatorname{Re}\left(\left(\mathrm{D}^{*}\right)^{T} \eta\right) \geq \operatorname{Rc}\left(\left(\mathrm{D}^{*}\right)^{T} \mathrm{Z}\right)+\frac{1}{2}|\mathrm{D}|^{2}
$$

in which - denotes conjugation and ${ }^{T}$ denotes transposition. The random variable on the lelthind side of equation (4.7) is a zero mean complex Gaussian random variable whl sariance $\frac{y_{a}}{i}|\mathbf{D}|^{2}$. As a result. if one conditions on the interuser interference $Z$. 
the resulting probability of error can be expressed as

$$
\text { Prob [error event } \begin{aligned}
\mathbf{D} \mid \mathbf{Z}] & =Q\left(\frac{\left.\operatorname{Re}\left(\mid \mathbf{D}^{-}\right)^{T} \mathbf{Z}\right)+\frac{1}{\mid}|\mathbf{D}|^{2}}{\sqrt{\frac{N_{0}}{2}|\mathbf{D}|^{2}}}\right) \\
& =Q\left(\sqrt{\frac{|\mathbf{D}|^{2}}{2 N_{0}}}+\sqrt{\frac{2}{N_{0}}} \frac{\operatorname{Re}\left(\left(\mathbf{D}^{-}\right)^{T} \mathbf{Z}\right)}{\sqrt{|\mathbf{D}|^{2}}}\right)
\end{aligned}
$$

In equation (4.8) the numerator of the second term of the Q-function san be definced as

$$
\begin{aligned}
& \mathbf{V}=\operatorname{Re}\left(\left(\mathbf{D}^{*}\right)^{\boldsymbol{T}} \mathbf{Z}\right) \\
& =\sum_{r=0}^{N-1}\left[D_{l_{p}} Z_{l_{p}}+D_{Q_{p}} \ddot{Q}_{Q_{p}}\right]
\end{aligned}
$$

in which $Z_{I_{p}}$ and $Z_{Q}$, (the user subscript ' $\mathrm{i}$ ' has been dropperl without losss of gent erality), are the in-phase and quadrature components of the intertuser interferrence random variable $Z_{p}$ at symbol time 'p' (the normalized forms are as defined in erpint tions (D.1:) and (D.12)). Furthermore $D_{l_{p}}$ and $D_{Q_{p}}$ are the in-phase and quadrat ure components of the error vector (i.e. $D_{p}=\sqrt{E_{S}}\left(d_{p_{p}}+d_{Q_{p}}\right.$ ) in which $d_{l_{p}}$ and $d_{Q_{p}}$ arc the in-phase and quadrature components respertively). Ciemetrically it is interest ing to note that $V$ is $\sqrt{\frac{2}{N_{0}}} \times$ the projection (in $N$-dimensional Euclidean space) of $Z_{I}+Z_{Q}$ onto $D_{I}+D_{Q}$. An alternate formulation of cyuation (4.9) that will prover expedient in he subsequent analysis is

$$
\mathrm{V}=\frac{\left(\mathrm{D}^{*}\right)^{T} \mathbf{Z}+\left(\mathrm{Z}^{*}\right)^{T} \mathrm{D}}{2}
$$

Fiom equations (1.8) and (3.14) one can express the exart probability of an event error as

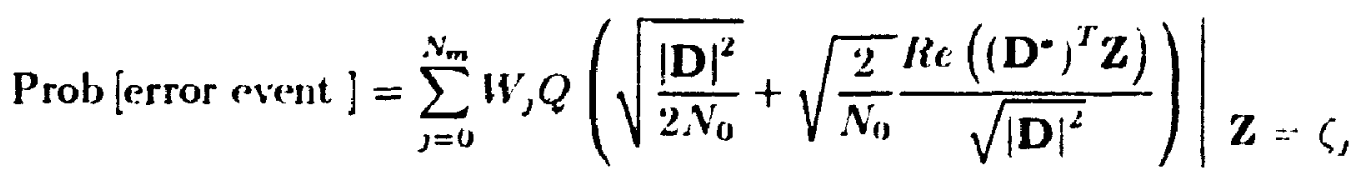




\subsection{Chernoff Bound Pairwise Error Probability}

It is well known that the pairwise error between any two events can be bounded using the ('hernoff bound which is defined by[36]

$$
\operatorname{Proh}(\mathbf{X}>0)<E[\exp (\lambda \mathbf{X})]
$$

in which $X$ is a random vertor. Similar to equation (2.23) the channel output signal in vectur form is

$$
\mathbf{Y}=\mathbf{X}+\mathbf{Z}+\eta
$$

The random vector " is a complex Gaussian random vector (the components of which have zero mean and variance of $\sigma^{2}$ ), and $Z$ is the complex vector of interuser interforence.

The pairwise crror probability $P(X \rightarrow \tilde{X})$ is the probability of choosing the coded serpuence $\overline{\mathbf{X}}$ instead of the coded sequence $\mathbf{X}$. This can be expressed in terms of ionditional probahilition as

$$
\operatorname{p.v}(\mathbf{Y} \mid \overline{\mathbf{X}})>\operatorname{p.v}(\mathbf{Y} \mid \mathbf{X})
$$

in which.$M$ denotes the dimensionality of the probability density function. The decoding pr cess employed by the Iiterbi Algorithm calculates the optimum path at aach node in the trellis by determining which path has the best metric (defired by m(Y.X)). The metric chosen for use in the Viterbi Algorithm depends on the optimality criterion of the decoder. Since the receiver under consideration is a correlator. and as such matched to an AlVGN channel, the metric employed is the maximum likelihosod metric

$$
m(\mathbf{Y}, \mathbf{X})=\ln \left[p_{. \mathbf{v}}(\mathbf{Y} \mid \mathbf{X})\right]
$$


which is optin um for a correlator receiver. However the conditional probability density function $p_{N}(Y \mid X)$ cannot in general be expressed in closed form since the probability density function of the random the vertor $Y$ is a convolution of the prob. ability density functions of a Gaussian random vector $\boldsymbol{\eta}$ and the interuser interference random vector $Z$ (having deterministic moments).

If an estimate of the random vector $\mathbf{Z}$ was available in each syminsl perioxl, once could define the metric with side information

$$
m(\mathbf{Y}, \mathbf{X}: \mathbf{Z})=\ln \left[\boldsymbol{p}_{N}(\mathbf{Y} \mid \mathbf{X}, \mathbf{Z})\right]
$$

In general the random variables $Z_{p}$ conprising $Z$ are dependert from one symlxol period to the next due to common interfering symbols from other users. The effert of the dependence can be bounded. and if required can easily be climinaterl by use. of interleaving and de-interleaving. Thus for the purpose of the present discussion it will be assumed that the $Z_{p}$ 's are independent from one symbol period to the next.

If no side information is available, the conditional density of equation (4.14) becomes

$$
\begin{aligned}
p_{N}(\mathbf{Y} \mid \mathbf{X}) & =\prod_{p=1}^{N} p\left(Y_{p} \mid X_{p}\right) \\
& =\prod_{p=1}^{N} \frac{1}{\sigma \sqrt{2 \pi}} \exp \left\{-\frac{\left|Y_{p}-X_{p}\right|^{2}}{2 \sigma^{2}}\right\}
\end{aligned}
$$

in which $Y_{p}$ is given by equation (4.13). Furthermore, under the abowe assumption of independence the metric $m(\mathbf{Y}, \mathbf{X})$ becomes

$$
m(\mathbf{Y}, \mathbf{X})=\sum_{p=1}^{N} m\left(Y_{p}, X_{p}\right)
$$

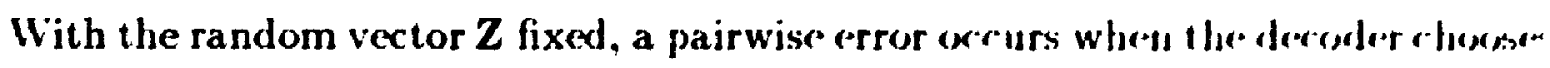


the sequence $\overline{\mathbf{X}}$ over the sequence $\mathbf{X}$ or equivalently when

$$
\sum_{p=1}^{N} m\left(Y_{p}, \tilde{X}_{p}\right)>\sum_{p=1}^{N} m\left(Y_{p}, X_{p}\right)
$$

Equivalently the probability of a pairwise error conditioned on the random variable $\mathbf{Z}$ is

$$
P(\mathbf{X} \rightarrow \dot{\mathbf{X}} \mid \mathbf{Z})=\operatorname{Prob}\left\{\sum_{p=1}^{N} m\left(Y_{p}, \dot{X}_{p}\right)>\sum_{p=1}^{N} m\left(Y_{p}, X_{p}\right)\right\}
$$

Applying the Chernoff bound of equation (4.12) to the right hand side of equation (4.20) results in

$$
P(\mathbf{X} \rightarrow \overline{\mathbf{X}} \mid \mathbf{Z}) \leq \prod_{p \in \nu} \mathrm{E}\left\{\exp \left[\lambda\left(m\left(Y_{p}, \tilde{X}_{p}\right)-m\left(Y_{p}, X_{p}\right)\right)\right]\right\}
$$

in which $\nu$ is the set of all $p$ such that $X_{p} \neq \dot{X}_{p}$. Furthermore from equation (4.17)

$$
m\left(Y_{p}, X_{p}\right)=\ln \left[p\left(Y_{p} \mid X_{p}\right)\right]=-\left|Y_{p}-X_{p}\right|^{2}
$$

in which the factor $\frac{1}{2 \sigma^{2}}$ has been incorporated into the parameter $\lambda$ without loss of generality, since the Chernoff bound will subsequently be optimized with respect to 1. Substituting equation (4.22) into equation (4.21) one obtains

$$
P(\mathbf{X} \rightarrow \overline{\mathbf{X}} \mid \mathrm{Z}) \leq \prod_{p \in \nu} \mathrm{E}\left\{\exp \left[\lambda\left(\left|Y_{p}-X_{p}\right|^{2}-\left|Y_{p}-\tilde{X}_{p}\right|^{2}\right)\right]\right\}
$$

Simplifying g ves

$$
P(X-\dot{X} \mid Z) \leq \prod_{p \in v} E\left\{\exp \left[\lambda\left(\left|Y_{p}\right|^{2}-\left|\dot{X}_{p}\right|^{2}-2 \operatorname{Re}\left\{Y_{p}\left(X_{p}-\dot{X}_{p}\right)^{*}\right\}\right)\right]\right\}
$$

Substituting equation ( 4.13 ) into equation (4.24) gives

$$
\begin{aligned}
& P(\mathbf{X} \rightarrow \tilde{\mathbf{X}} \mid Z) \leq \prod_{p \in \nu} \mathbf{E}\left\{\operatorname { e x p } \left[\lambda \left(\left|X_{p}\right|^{2}-\left|\tilde{X}_{p}\right|-\right.\right.\right. \\
&\left.\left.\left.2 \operatorname{Re}\left\{\left(X_{p}+\eta_{p}+Z_{p}\right)\left(X_{p}-\tilde{X}_{p}\right)^{*}\right\}\right)\right]\right\} \\
&=\prod_{p \in \nu} \exp \left(-\lambda E_{o}\left|x_{p}-\tilde{x}_{p}\right|^{2}\right) \times \\
& \mathbf{E}\left\{\exp \left[-2 \lambda \sqrt{E_{s}} \operatorname{Re}\left\{\left(\eta_{p}\left(x_{p}-\tilde{x}_{p}\right)^{*}\right)\right]\right\} \times\right. \\
& \mathbf{E}\left\{\exp \left[-2 \lambda E, \operatorname{Re}\left\{\left(z_{p}\left(x_{p}-\tilde{x}_{p}\right)^{*}\right\}\right]\right\}\right.
\end{aligned}
$$


in which $z_{p}=Z_{p} / \sqrt{ } E_{g}$. The first expectation of equation (4.25) involving the coill. plex Gaussian variable $\eta_{p}$ can be evaluated by expressing $\eta_{p}$ in terms of its cal and imaginary components. Denote the real (i.e. in-phase) component of $\eta_{p}$ by $\eta_{l}$ and the :maginary (i.e. quadrature) component by $\eta_{Q}$, in which the subscript $p$ has been dropped without loss of generality. Furthermore define the normalized error vector as $d=x-\tilde{x}$ (the norm of which is the distance between $x$ and $\tilde{x}$ ), and as in the case of $\eta_{p}$ define the real and imaginary components of $d$ as $d_{l}$ and $d_{Q}$. Noting that $\eta_{1}$ and $\eta_{Q}$ are uncorrelated, cero mean Gaussian random variables, each with variance $N_{0} / 2$, one obtains

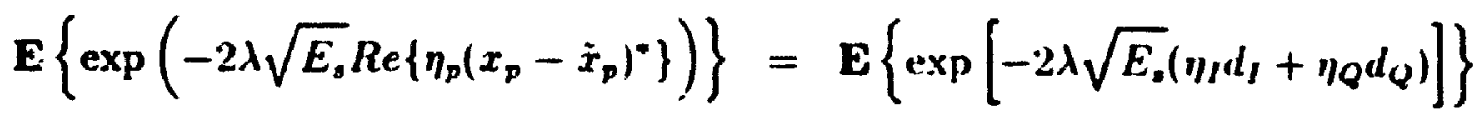

$$
\begin{aligned}
& =\frac{1}{\sqrt{N_{0} \pi}} \int_{-\infty}^{\infty} \exp \left\{-\frac{\eta_{1}^{2}}{N_{0}}-2 \lambda \sqrt{E_{s} \eta_{1} d_{l}}\right\} d \eta_{!} \times \\
& \frac{1}{\sqrt{N_{0 \pi}}} \int_{-\infty}^{\infty} \exp \left\{-\frac{\eta_{Q}^{2}}{V_{0}}-2 \lambda \sqrt{E_{s} \eta_{Q} d Q}\right\} d r_{i n}
\end{aligned}
$$

Completing the square in each integral and using the fact that the resulting densit: integrates to $r$,e, gives

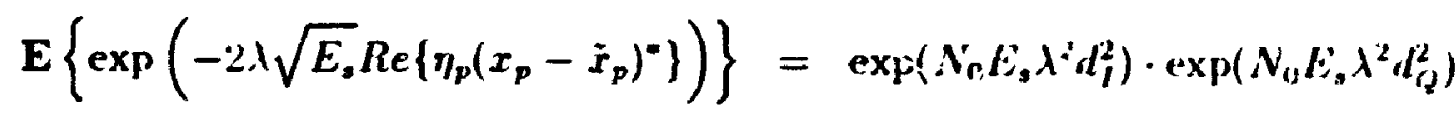

$$
\begin{aligned}
& =\exp \left(N_{0} E, \lambda^{2}{ }_{i p}-\left.\dot{x}_{p}\right|^{2}\right)
\end{aligned}
$$

It should be noted that the result of equation (4.27) also follows from the definition of the characteristic function of: Caussian random variable. The second expretal ias of equation (4.25) can be writt as

$$
\left.\mathbf{E}\left\{\exp \left(-2 \lambda E_{q} \operatorname{Re}\left\{z_{p}\left(x_{p}-\dot{x}_{p}\right)^{*}\right\}\right)\right\}=\int_{-\infty}^{\infty} \int_{-\infty}^{\infty} \exp \left(-2 \backslash E_{s} \mid z_{1} d d_{1}+z_{Q} d d_{Q}\right\}\right) .
$$

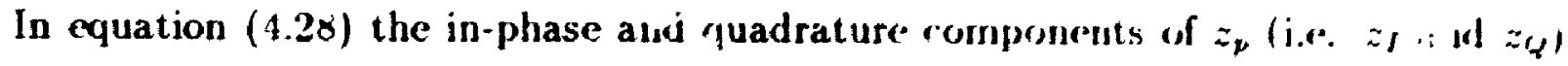



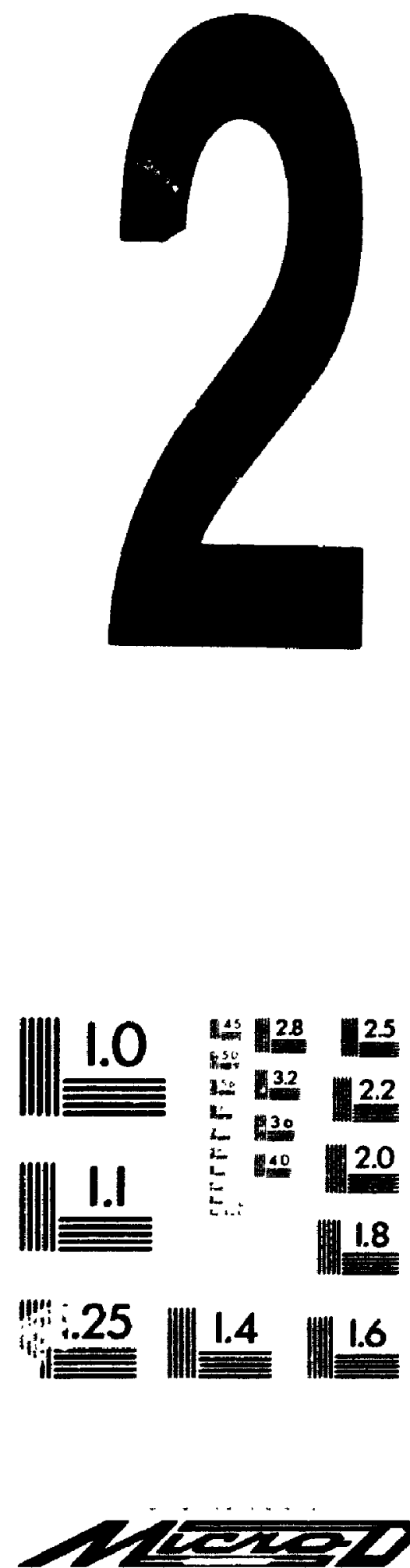
are dependent (see Appendix $G$ ) and as such the moments must be evaluated jointly (i.e. the density $p\left(z_{I}, z_{Q}\right)$ cannot be split into individual density functions of $z_{I}$ and $\left.z_{Q}\right)$. Defining $v=z_{1} d_{I}+z_{Q} d_{Q}\left(V=E_{0} \cdot v\right.$, i.e. see equation (4.10)), equation (4.28) can be rewritten as

$$
\begin{aligned}
E\left\{\exp \left(-2 \lambda E_{s} \operatorname{Re}\left\{z_{p}\left(x_{p}-\dot{x}_{p}\right)^{-}\right\}\right)\right\} & =\int_{-\infty}^{\infty} \exp \left(-2 \lambda E_{s} v\right) p(v) d v \\
& =\sum_{i=1}^{N_{m}} W_{p_{0}} \exp \left(-2 \lambda E_{s} \zeta_{p_{0}}\right)
\end{aligned}
$$

in which $\zeta_{p_{i}}$ is the $i^{\text {th }}$ node corresponding to the $i^{\text {th }}$ weight $W_{p_{i}}$. The number of moments used in the weighted summation is $N_{m}$. Substituting the results of equations (4.29) and (4.27) into equation (4.25) gives a pairwise error of

$$
\left.P(\mathrm{x} \rightarrow \overline{\mathbf{x}}) \leq \prod_{p \in \nu} \exp \left[-\lambda E_{9}\left(1-\lambda N_{0}\right)\left|x_{p}-\dot{x}_{p}\right|^{2}\right)\right] \cdot \sum_{i=1}^{N_{m}} W_{p_{0}} \exp \left(-2 \lambda E_{s} \zeta_{p_{\mathrm{s}}}\right)
$$

In order to obtain the tightest possible upper bound on the probability of error. equation (4.30) must be optimized with respect to the Chernoff ;)arameter $\lambda$. This can more easily be achioved if $\lambda$ is redefined as $\lambda^{\prime} / N_{0}$. Dropping the primes, gives

$$
\left.P(\mathrm{x} \rightarrow \tilde{\mathbf{x}}) \leq \prod_{p \in \nu}\left[\exp \left[-\frac{E_{g}}{N_{0}} \lambda(1-\lambda)\left|x_{p}-\tilde{x}_{p}\right|^{2}\right)\right] \cdot \sum_{i=1}^{N_{m}} W_{p_{t}} \exp \left(-2 \lambda \frac{E_{s}}{N_{0}} \zeta_{p_{0}}\right)\right]
$$

Expressing the pairwise error bound as in equation (4.31) enables $\lambda$ to be optimizerd in terms of $E_{g} / N_{0}$, a common system input parameter. A point of note is that the $\lambda$ of equation (4.31) is in fact the same $\lambda$ as in the original Chernoff bound, allowing easy tracking of the deviation of the optimum value of $\lambda$ from the Bhattacharyya value of $\frac{1}{2}$. A second point of note is that the optimization of $\lambda$ is dependent on the length $N$ of the error event. Since the transfer function bound involves calculating the error events for all possible lengths, it is not practically possible to calculate the optimum Chernoff bound. In this study, $\lambda$ was optimized over the branch with the minimum distance. and this value was employed for all branches. The degradation of the tightness of the Charnoff bound was not that significant for the corles that were considered. 


\subsection{Chernoff Bound Using a Gaussian Approxi- mation}

A common assumption that is used to simplify the analysis of the interuser interference in a SSMA system is to assume that such interference is Gaussian. If this assumption is used to evaluate the expectation with respect to $z_{p}$ in equation (4.25) one obtains the approximation

$$
\mathrm{E}\left\{\exp \left(-2 \lambda E_{s} \operatorname{Re}\left\{z_{p}\left(x_{p}-\tilde{x}_{p}\right)^{*}\right\}\right)\right\} \approx \exp \left(2 \lambda^{2} E_{s}^{2} \sigma_{z}^{2}\left|x_{p}-\tilde{x}_{p}\right|^{2}\right)
$$

in which $\sigma_{z}^{2}=\frac{1}{2} \mathrm{E}\left[\left|z_{p}\right|^{2}\right]$ (i.e. the variance of the interference in the in-phase or quadrature channels). In arriving at the result of equation (4.32) it was assumed that $z_{l}$ and $z_{Q}$ wure independent, which is a valid assumption since if $z_{I}$ and $z_{Q}$ are jointly Gaussian and uncorrelated (by definition) then it follows that they are independent. Substituting the results of equations (4.32) and (4.27) into equation (4.25) one obtains the following approximate pairwise error bound

$$
P(\mathrm{x} \rightarrow \tilde{\mathrm{x}}) \leq \prod_{p \in \nu} \exp \left\{-\lambda E_{s}\left(1-2 \lambda\left(N_{0} / 2+\sigma_{z}^{2} E_{\bullet}\right)\right)\left|x_{p}-\tilde{x}_{p}\right|^{2}\right\}
$$

Optimizing equation (4.33) with respect to the Chernoff parameter $\lambda$ gives

$$
\lambda=\frac{1}{4\left(N_{0} / 2+\sigma_{z}^{2} E_{8}\right)}
$$

Substituting equation (4.34) into equation (4.33) gives a resulting bound of

$$
\begin{aligned}
P(\mathbf{x} \rightarrow \tilde{\mathbf{x}}) & \leq \prod_{p \in \nu} \exp \left\{-\frac{\left|x_{p}-\tilde{x}_{p}\right|^{2} E_{s}}{8\left(N_{0} / 2+\sigma_{z}^{2} E_{s}\right)}\right\} \\
& =\prod_{p \in \nu} \exp \left\{-\frac{\left|x_{p}-\tilde{x}_{p}\right|^{2}}{4\left(\frac{N_{0}}{E_{o}}+\mathbf{E}\left[\left|z_{p}\right|^{2}\right]\right)}\right\}
\end{aligned}
$$

$A$ point to note is that the bound obtained in equation (4.35) is in fact the Bhattacharyya bound for the Gaussian approximation. This result is comparable to one derived by Pursley[12] for uncoded binary SSMA. 


\section{Chapter 5}

\section{Performance Results in an AWGN Channel}

Employing the generalized transfer function bound algorithm described in Chapter 3 in conjunction with the Chernoff bounds for pairwise errors analyzed in Chapter 4, the performance of various trellis and convolutionally coded SSMA systems have been evaluated in an AWGN channel. These results are presented in this chapter. However, before proceeding to present these results it is necessary to benchmark the accuracy of the method of moments algorithm that has been employed in the evalu.. .on of thes" codes. Furthermore the validity of the assumption that the interuser interference can be treated as being independent from one symbol period to the next will be examined. The tightness of the resulting Chernoff bound with respect to the actual performance will also be treated through comparison to the performance of transfer function bounds employing the Q-function for pairwise error probalility evaluation (as opposed to the Chernoff bound), as well as through comparison to the results of Monte Carlo simulations (presented in a later subsection). 


\subsection{Benchmark of Method of Moments}

As was cited in the literature survey of Chapter 1 , the method of moments algorithm has been employed by several previous researchers to evaluate the performance of SSMA systems in an AWGN environment[2][22]. In addition to the method of moments, there have been numerous other approximation techniques employed for the evaluation of SSMA performance of uncoded systems[12][13][14]. In order to benchmark the accuracy of the method of moments results, as well as to ascertain the number of moments required to obtain accurate results, the performance of uncoded SSMA systems employing Maximal length (M) sequences of length 31 , with $K=2$ and $K=3$ users was evaluated. The performance of these same systems has been previously evaluted by Pursley et. al.[14] using bounding techniques. The generator polynomials of the three M-sequences that were employed are (in octal notation) 45, 67 and 75. Furthermore the generators were choosen to have auto-optimal/least sidelobe energy (AO/LSE) initial loadings[40] of 63, 70 and 57 respectively. For the system with 2 users (generators 45 and 67), the performance of the method of moments is also compared to upper and lower bounds of the probability of bit error rate performance (see Table IV of [14]) in Table 5.1. Similar results are shown in Table 5.2 for the system with 3 users (compare to Table $V$ of [14]). From these tables it can be sien that the method of moments provides accurate results up to two to thrce decimal places which is more than sufficient when calculating probability of error values. The results for the method of moments were obtained from using 20 moments in the evaluation. It has been found that for the codes evaluated in this study that 20 to 22 moments usually provides results that have the desired accuracy of three decimal places. 


\begin{tabular}{||c|c|c|c||}
\hline \hline \multirow{2}{*}{$E_{b} / N_{0}$} & \multicolumn{3}{|c||}{ Probability of bit error } \\
\cline { 2 - 4 } & Lower Bound & Upper Bound & Method of Moments \\
\hline \hline 4 & $1.44 \times 10^{-2}$ & $1.45 \times 10^{-2}$ & $1.443 \times 10^{-2}$ \\
6 & $3.35 \times 10^{-3}$ & $3.36 \times 10^{-3}$ & $3.34 i \times 10^{-3}$ \\
8 & $4.42 \times 10^{-4}$ & $4.24 \times 10^{-4}$ & $4.216 \times 10^{-4}$ \\
10 & $2.40 \times 10^{-5}$ & $2.42 \times 10^{-5}$ & $2.397 \times 10^{-5}$ \\
12 & $4.81 \times 10^{-7}$ & $4.89 \times 10^{-7}$ & $4.815 \times 10^{-7}$ \\
14 & $2.28 \times 10^{-9}$ & $2.34 \times 10^{-9}$ & $2.285 \times 10^{-9}$ \\
\hline \hline
\end{tabular}

Table 5.1: Performance of SSMA system with $K=2$ and $M$-sequences of length 31

\begin{tabular}{||c|c|c|c||}
\hline \hline \multirow{2}{*}{$E_{b} / N_{\mathrm{c}}$} & \multicolumn{3}{|c||}{ Probability of bit error } \\
\cline { 2 - 4 } & Lower Bound & Upper Bound & Met hod of Moments \\
\hline \hline 4 & $1.66 \times 10^{-2}$ & $1.69 \times 10^{-2}$ & $1.662 \times 10^{-2}$ \\
6 & $1.58 \times 10^{-3}$ & $4.77 \times 10^{-3}$ & $1.596 \times 10^{-3}$ \\
8 & $8.43 \times 10^{-4}$ & $9.11 \times 10^{-4}$ & $8.487 \times 10^{-4}$ \\
10 & $1.06 \times 10^{-4}$ & $1.21 \times 10^{-4}$ & $1.076 \times 10^{-4}$ \\
12 & $1.04 \times 10^{-5}$ & $1.29 \times 10^{-5}$ & $1.081 \times 10^{-5}$ \\
14 & $9.68 \times 10^{-7}$ & $1.33 \times 10^{-6}$ & $1.047 \times 10^{-6}$ \\
\hline \hline
\end{tabular}

Table 5.2: Performance of SSMA system with $K=3$ and M-seguences of length 31 


\subsection{Validity of the Independence Assumption}

Due to the asynchronous nature of the interuser interference (IUI) tor the SS systems under consideration. the IUI random variable $Z_{p}^{\prime}$ is not independent from one symbol period to the next. This can be easily discerned from equation ( 2.21$)$, from which it is evident that both $Z_{p}^{;}$and $Z_{p+1}^{i}$ are a function of the transmitted symbol $x_{p}^{i}$. Since the method of moments involves calculating the moments of the random variable $Z_{p}^{*}$ up to some arbitrary order $\mathrm{V}_{m}$, the moments of the IUI over a symbol sequence of length .$V$ must be evaluated jointly. Ilowever if the moments over each symbol period were independent the moments of the IUI would simply be the product of the moments of the individual symbols. The accuracy and validity of assuming such independence is the topic of this section. The algebraic expresssions for the evaluation of the moments of a sequence of arbitrary length are provided in Appendix $A$. In general terms of the Chernoff bound of equation (4.12), the comparison is between

$$
\begin{aligned}
& \mathbf{E}[\exp \{\lambda[m(\mathbf{Y} . \dot{\mathbf{X}})-m(\mathbf{Y}, \mathbf{X})]\}] \\
& \text { versus } \\
& \prod_{n \in \eta} E\left\{\exp \left\{\lambda\left(m\left(Y_{n}, \dot{I}_{n}\right)-m\left(Y_{n}, X_{n}\right)\right)\right]\right\}
\end{aligned}
$$

A comparison of the above bounds for several arbitrary sequence pairs is plotted versus $\frac{F_{*}}{x_{0}}$ for path lengths of 2 and 3 in Figure 5.1. For sequence lengths of 4 or greater. the calculation of the dependent moments becornes prohibitively time consuming. From the above figures it can be seen that the use of the independence assumption for short path lengths upper bounds the true bound calculated from the rependent moments. 


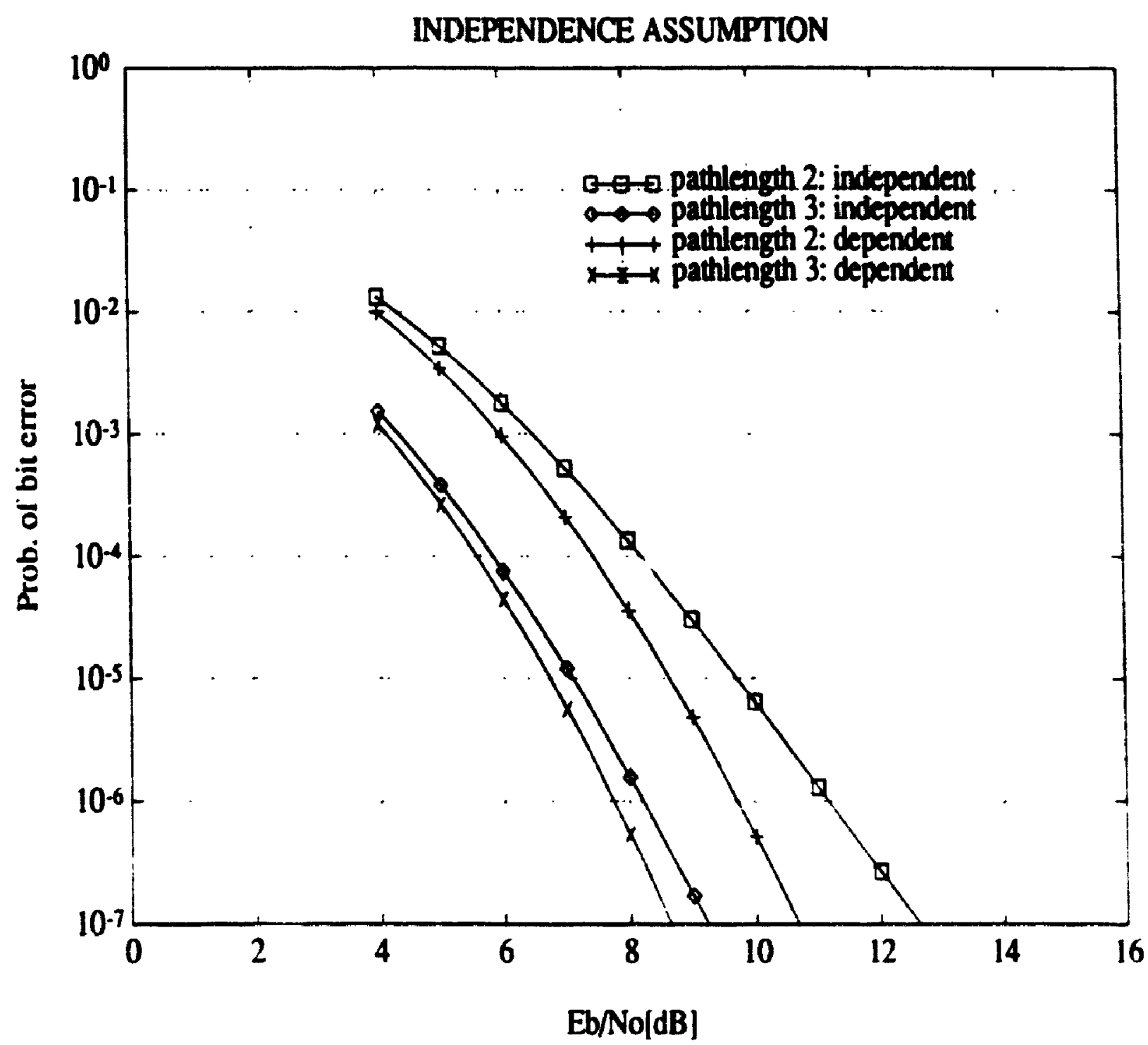

Figure 5.1: A comparison of the Chernoff bound for dependent versias independent. 1.uments for path lengths of 2 and 3 


\subsection{Coded Q-function Bound}

For a trellis coded system operating solely in an AWGN channel (i.e. with no IUI), it is nossible to tighten the generalized transfer function bound of equation (3.9) by ampluging the inequality [91][109]

$$
Q(\sqrt{x+y}) \leq Q(\sqrt{x}) \exp \left(-\frac{y}{2}\right)
$$

This results in an asymptotically tight bound of the form [88]

in which

$$
P_{h} \leq\left.\frac{1}{n} Q\left(\sqrt{\frac{n E_{b} d_{\text {free }}^{2}}{2 N_{0}}}\right) D^{-d_{f \text { free }}^{2}} \frac{\partial T(D . I)}{\partial I}\right|_{I=1}
$$

$$
D=\exp \left(-\frac{n E_{b}}{4 N_{0}}\right)
$$

and $d_{\text {free }}$ is the minimum distance of the code. Figure 5.2 illustrates a comparison of the bound of erration (5.3) with the Chernoff bound performance of equation (3.9), for the 4-PSK 4 tate code operating in ar. AWGN channel with no IUI. Also plotted in the same figure is a lower bound based on $d_{\text {free }}$ of the code. It can be seen that at a probability of bit error rate of $10^{-5}$ that the Chernoff bound performance is within $1 d B$ of the performance of the Q-function bound or the lower bound. Figure 5.3 illustrates an analogous set of curves for the rate $\frac{1}{2} 4$-state convolutional code of Table 3.7. These curves agree with the results presented in Heller and Jacobs[118], providing a benchmain for the validity of the transfer function bound algorithm. Again, for a probability of bit error rate of $10^{-5}$ the Chernoff bound is within $1 d B$ of the tighier $Q$-function bound. Such a correspondence between the $Q$-function bound and the Chernoff bound is also valid for the other codes considered in this study.

\subsection{Uncoded SSMA Bounds}

As wi. noted in Section 2.2 the trellis coded SSMA systems evaluated in this study 'mpoy the same PN sequence in both the in-phase and quadrature channels, as 


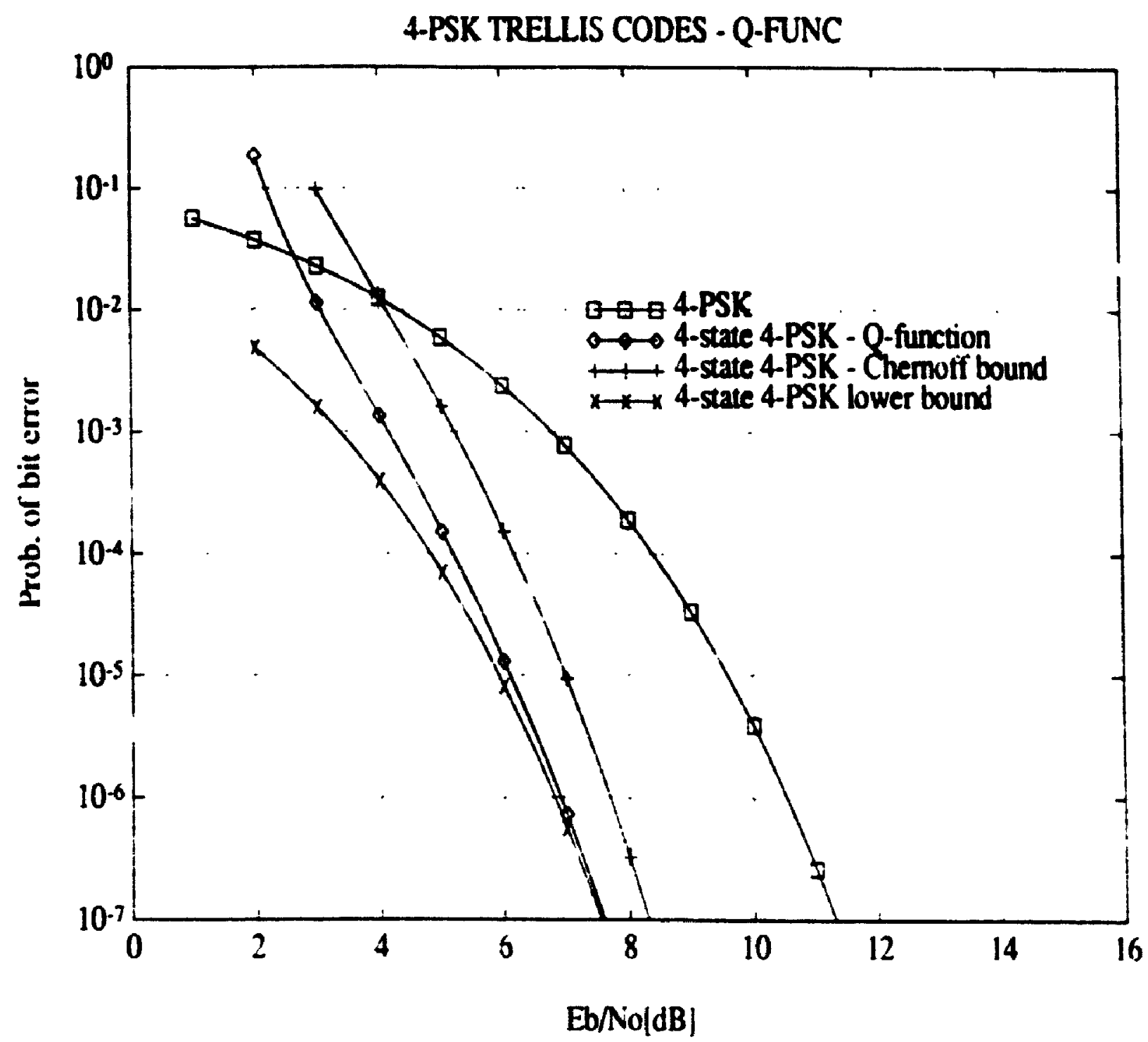

Figure 5.2: Performance of Q-function bound versis ('bernoff bound for the 4 state 4 -PSK trellis code. 


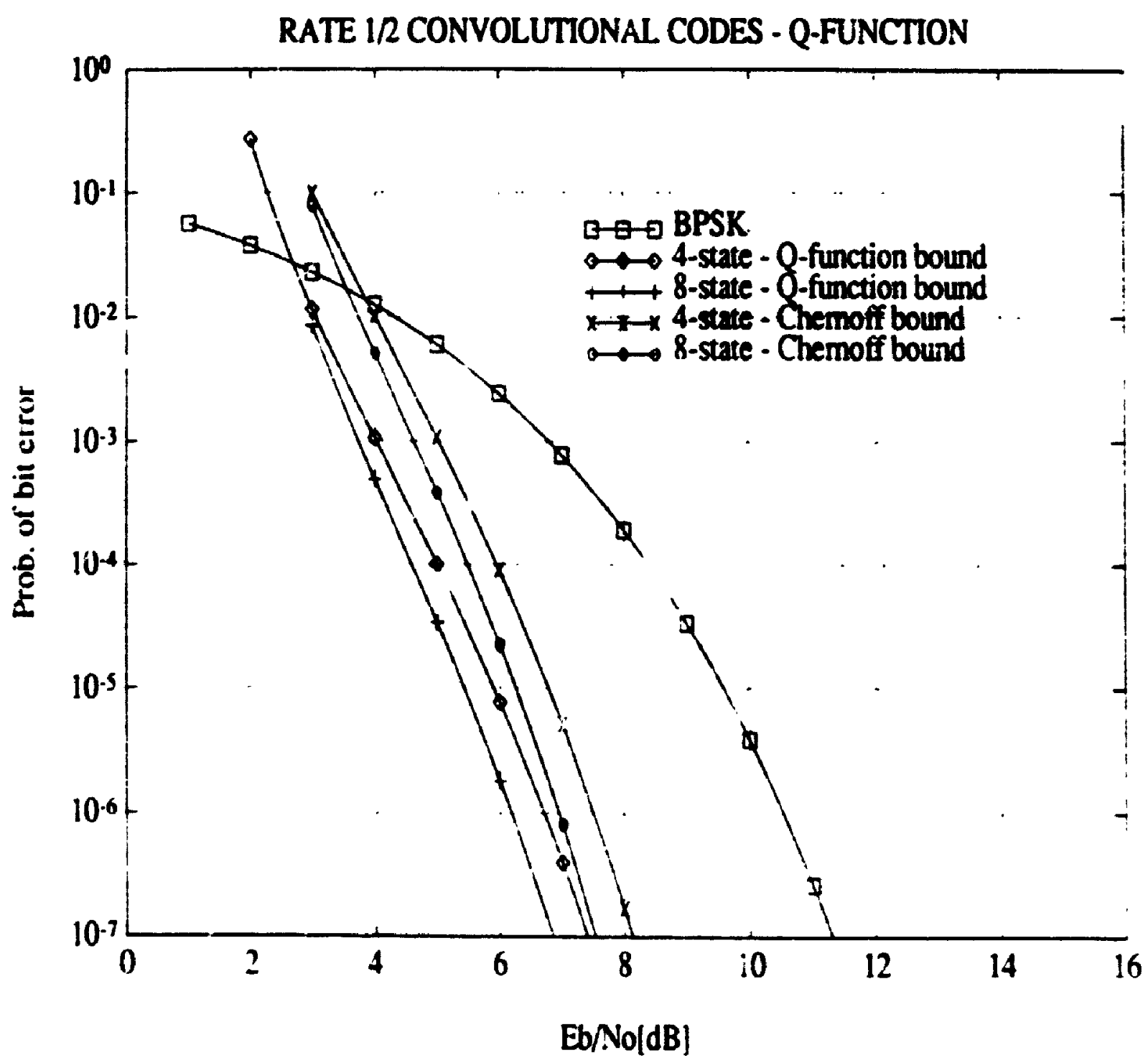

Figure 5.3: ('omparsion of Q-function bound versus Chernoff bound for 4 and 8 state rate $1 / 2$ convolutional codes 
opposed to Pursley's technique of employing different PN spreading sequences in both the in-phase and quadrature channels. As such. in order to evaluate the coding gain of the trellis and convolutionally coded SSMA systems. one inust benchmark their performance against the performance of uncoderl transmission possessing the same throughput as the coded systems for a given $\frac{E_{k}}{1}$. Thus the rate $1 / 2$ trellis codes and convolutional codes will be benchmarked against an t:ncoded IBPSh SSMA system; the rate $2 / n$ trellis and convolutional codes will be benchmarked against uncoded 4-PSK SSMA (i.e. QPSK with the same spreading sequence in the 1 and $Q$ channels): and finally the rate $3 / n$ codes will be compared to uncoled X-PSK SSMA. Figure 5.4 illustrates the performance of the aforementioned uncoled SEM $A$ systems for $h=10$ users. Whereas Figure 5.5 illustrates the analogous performance for $k=20$ users. It should be noted that the III of the SSIIA causes consideralsle degradation particularly for the 1-PSK and \&-PSK modulations. The performance of uncoded 8.PSK SSMA is $p$. ticularly poor. eren at high signal to roise ration (i.e. the probability of bit error saturates in the range of $10^{-2}$ to $10^{-3}$ for a modest numier of users in the range of $k=10$ to $k=20)$.

\subsection{Trellis Coded SSMA}

Beiore presenting the results of trellis coded SSMA systems. it will low use-ful t." review and expand upon several of the assumptions of the analysis in order to facilitate a proper understanding of the results. As was stated in the Introduction and in Chapter 2. the performance of the codes was evaluatorl on the basis of mpual complexity and throughput. and furthermore the chip rate is upper lesunderl (i.e. there is some minimum chip poriod $T_{r}^{\prime \prime}$ such that $T_{r}>T_{r}^{\prime}$ ). These assumptions will merit further discussion when the performance of the convolutionally conloul systems are examined. Another relevant assumption is that the inturforing signals (i.e. all other system e'sers) and the desired signal. all arrive at the reveiwer with the salll. absolute arrier power. 


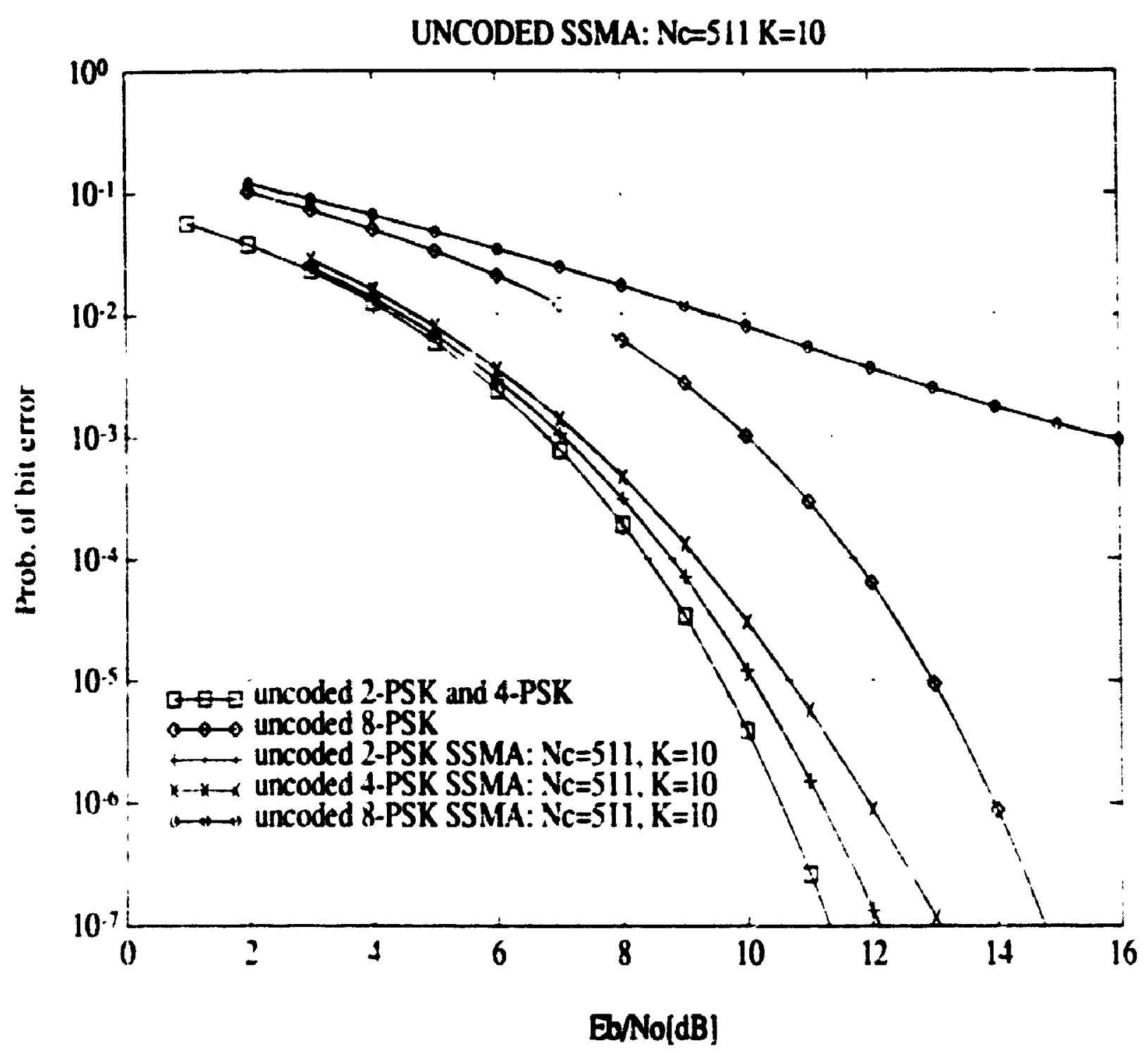

Fin.re 5.4: Performance of uncoded SSMA for $K=10$ users 


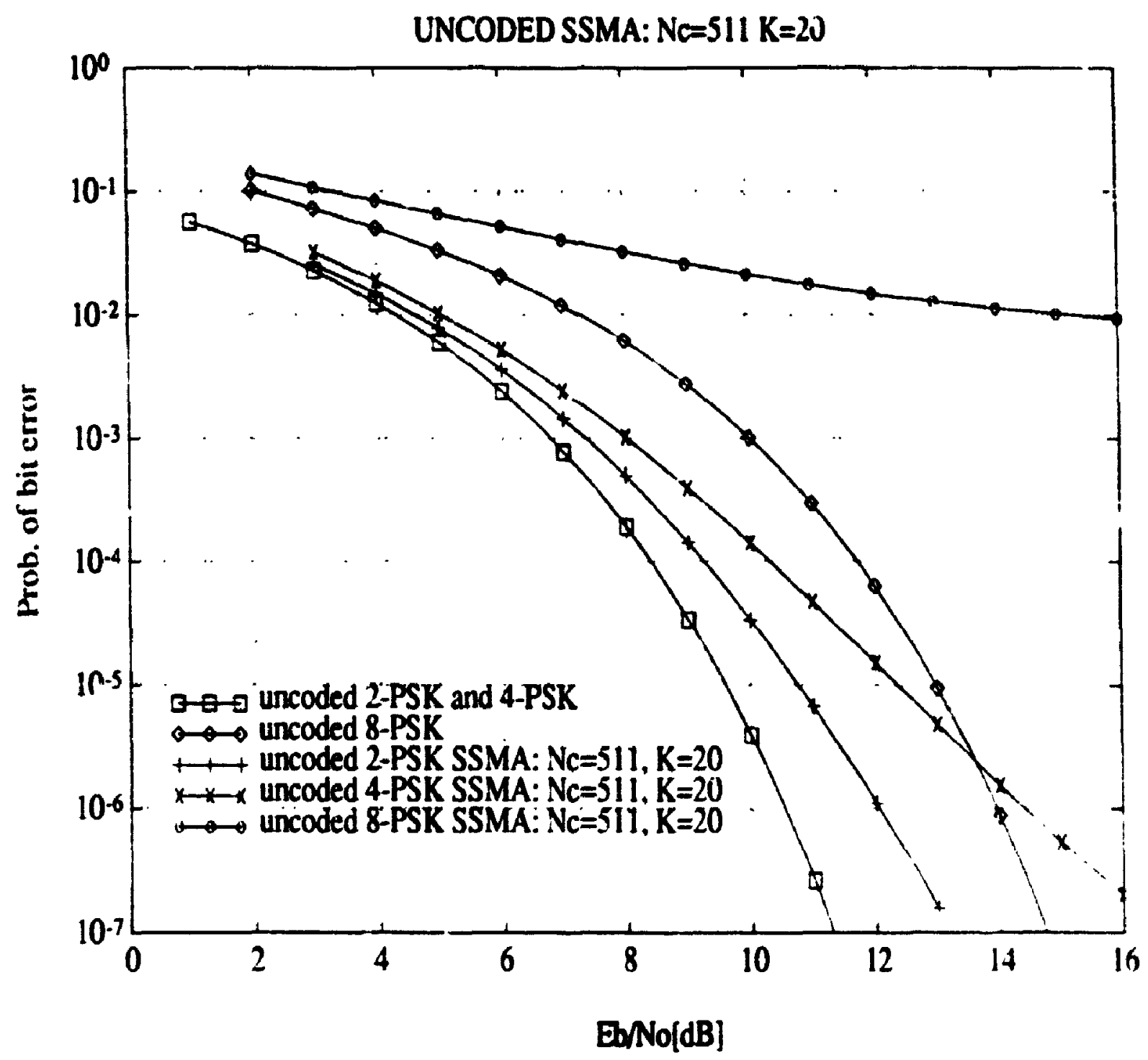

Figure 5.5: Performance of uncoded SSMA for $K=20$ users 
As was stated in section 5.3. the Chernoff bound performance typically upper bounds the true performance by $0.5 \mathrm{~dB}$ to $1 \mathrm{~dB}$ (the curves are in fact upper bounds on the average performance). In section 4.2 it was noted that the Chernoff parameter $\lambda$ was a function of the length of the error event under consideration. In fact, by examining equation (4.31) it can be seen that $\lambda$ is also a function of $\frac{E_{1}}{N_{0}}$, the symbol energy to noise spectral density ratio. I typical example of the optimum values of $\lambda$ as a function of $\frac{E_{p}}{v_{0}}$ is plotted in Figure 5.6 for the 4-PSK 4-state trellis code. This curve represents the optimum values of $\lambda$ for a branch of distance 2 . All of the following performance curves employed optimum values of $\lambda$ as a function of $E_{N_{0}}$ and the minimum branch distance in the evaluation of the Chernoff bounds of the code.

\subsubsection{4-PSK Codes}

Figures 5.7, 5.8 and 5.9 illustrate the performance of rate $\frac{1}{2}$ trellis codes. employing a 1-PSK signal constellation and having 2,4 and 8 states respectively. The encoders for these trellis codes can be found in Figures 3.1, 3.2 and 3.3 respectively. In each figure. the probability of bit error rate curves are plotted for the codes in the presence of: no IUI (i.e. just $A W C N$ ); $K=10$ multiple access users; and $K=20$ multiple access users. Also plotted for benchmark purposes are the performance curves of Incoded BIPSK SSMA systems having $K=10$ and $K=20$ users, respectively. All "f the treliis coded systems assume the use of Gold spreading sequences of length $V_{c}=311$ chips. These PN sequences are assumed to span one symbol period.

At a probability of bit crror rate of $10^{-5}$ it can be seen that the coded system otfers a gain over the uncoded systems varying from $1 \mathrm{~dB}$ for the 2-state code, to approximately $3 \mathrm{~dB}$ for the 4 -state code and $3.6 \mathrm{~dB}$ for the 8 -state code. The price paid for the incrense in coding gain is complexity. It should also be noted that for the nor complex codes (i.e. the 4 and 8 state codes), that the degradation of the performance as the number of users is increased from $K=10$ to $K=20$ users is less in 


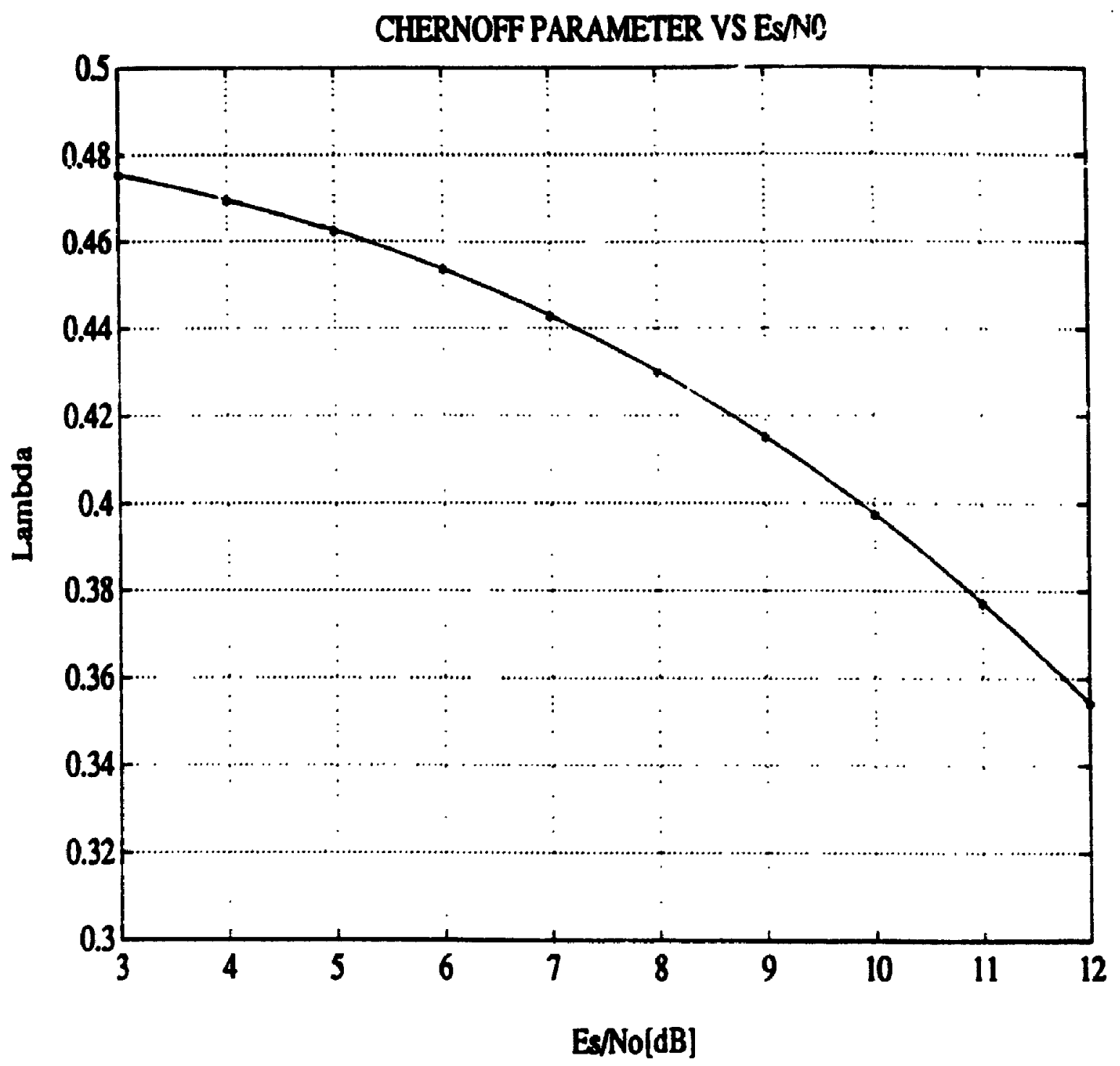

Figure 5.6: Chernoff parameter $\lambda$ as a function of $\frac{E_{0}}{N_{0}}$ for a branch of distance 2 . 


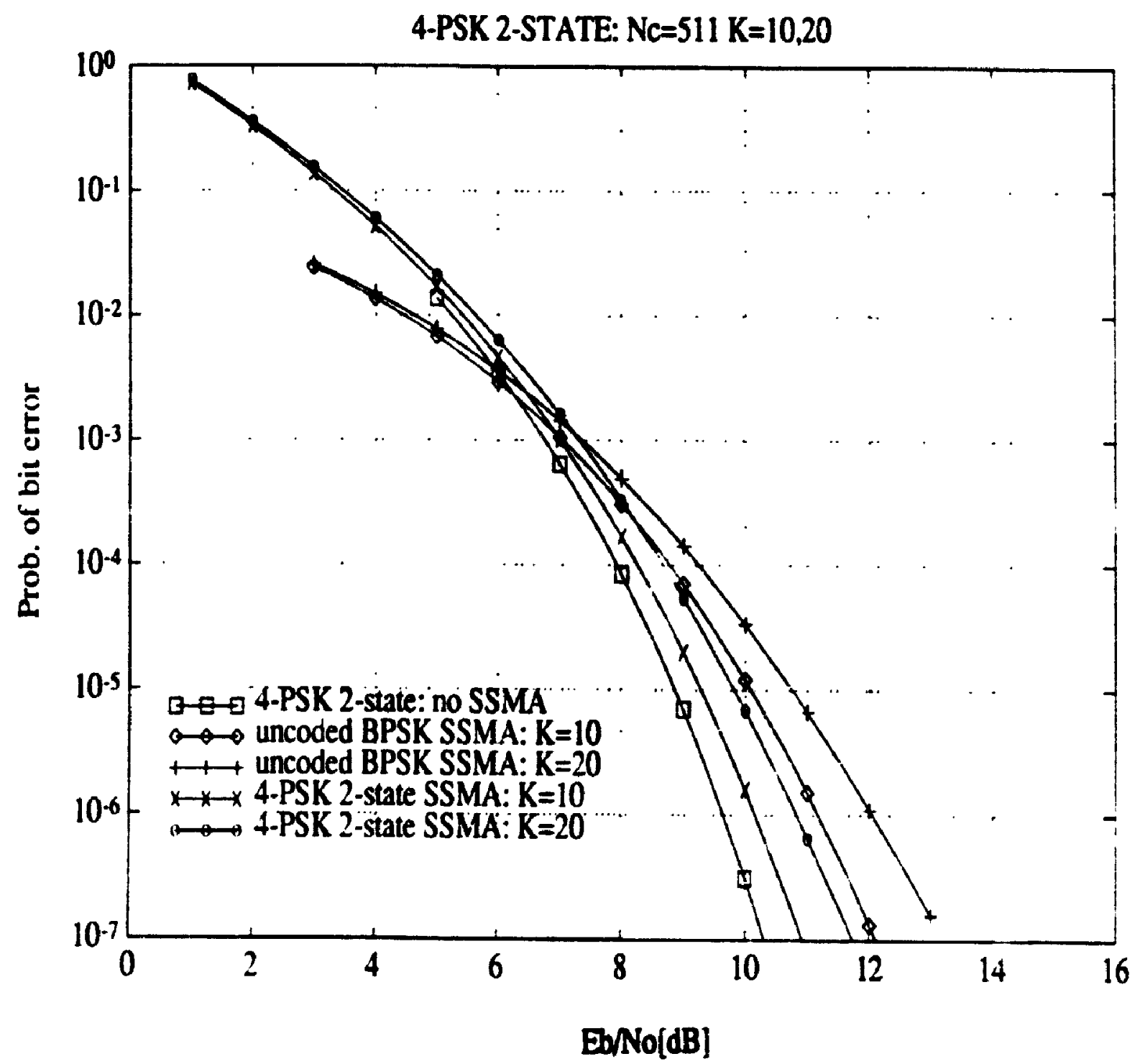

Figure 5.7: Performance of 4-PSK 2-state trellis codes for $K=10$ and $K=20$ users $\left(x_{i}=511\right)$. 


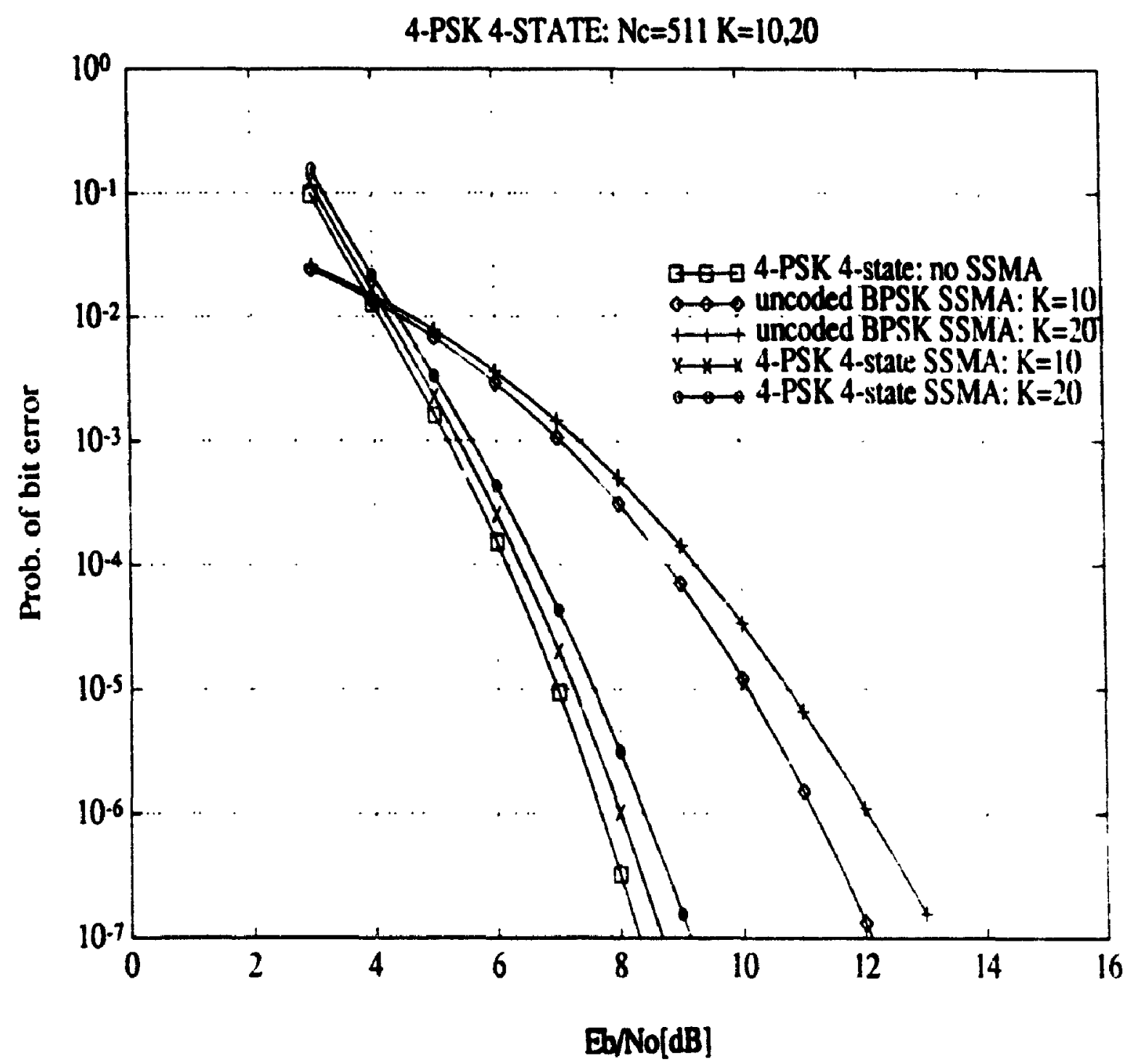

Figure 5.8: Performance of 4-PSK 4-state trellis codes for $K=10$ and $K=20$ users $\left(N_{c}=511\right)$. 


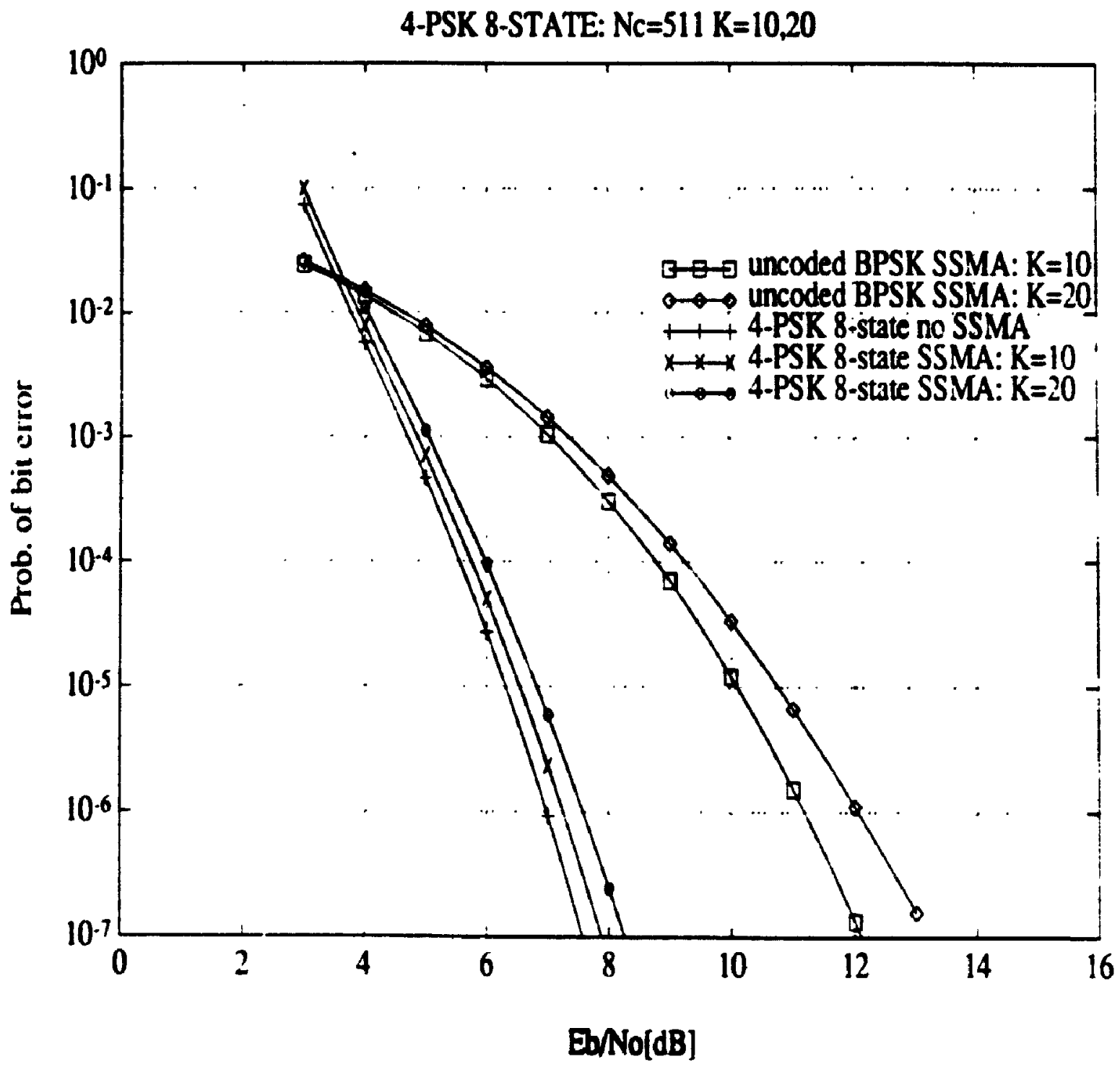

Figure 5.9: Performance of 4-PSK 8-state trellis codes for $K=10$ and $K=20$ users $\left(N_{c}=511\right)$. 
the coded case than in the uncoded case.

\subsubsection{8-PSK Codes}

Figures $5.10,5.115 .12$ and 5.13 illustrate the performance of rate $\frac{2}{3}$ trellis codrs, employing an 8-PSK signal constellation and having 2, 4, 8 and 16 states respectively. The encoders for these trellis codes can be found in Figures 3.1, 3.2. 3.4 and 3.5 respectively. As in the case for the 4-PSK trellis codes, in each figure the probability of bit error rate curves are plotted for the codes in the presence of: no IUI (i.e. just AWGN); $K=10$ multiple access users; and $K=20$ multiple access users. Also plotted for benchmark purposes are the performance curves of uncoded 4.PSk SSMA systems having $K=10$ and $K=20$ users respectively. Again all of the trellis coded systems assume the use of Gold spreading sequences of length $N_{c}=511 \mathrm{chips}$ and the PN sequences are assumed to span one symbol period. For $k=20$ users at a probability of bit error rate of $10^{-5}$ it can be seen that the coded system offers a gain over the uncoded systems varying from $2.8 \mathrm{~dB}$ for the 4 -state code, to appruximatcly $3.1 \mathrm{~dB}$ for the 8-state code and $3.7 \mathrm{~dB}$ for the 16-state code.

Since the 8-PSK trellis codes are rate $2 / 3$ codes, they have twice the throughput. of the rate $1 / 24$-PSK codes, for the same symbol rate. In order for the rate $1 / 2$ 4-PSK code to transmit at the same bit rate as the rate $2 / 3$ \&-PSK, une must dumble the symbol rate (and equivalently the bit rate, as well as the noise landwidth), of the 4-PSK code. This results in a penalty of over $3 \mathrm{~dB}$ since the noise bandwidth is doubled, and furthermore the PN spreading sequence length must also be lialverl since $T_{c}$ is assumed to be fixed. Under these conditions of comparison, the rate $2 / 3$ 8-PSK codes are superior to the rate $1 / 2$ 4-PSK codes. As an example, consider the performance of the 4 state codes for $K=10$ users, at a probability of lit arror

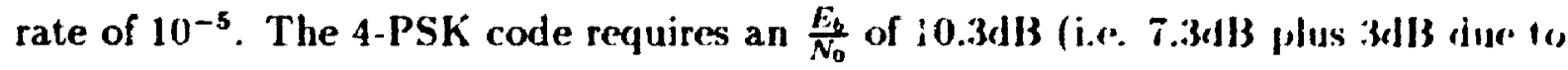
the increased noise bandwidth), whereas the 8-PSK codve requires an $\frac{k_{0}}{N_{0}}$,f x.fidls Accounting for the $3 \mathrm{~dB}$ loss of the rate $1 / 2$ code in order to soltain the same signalling 


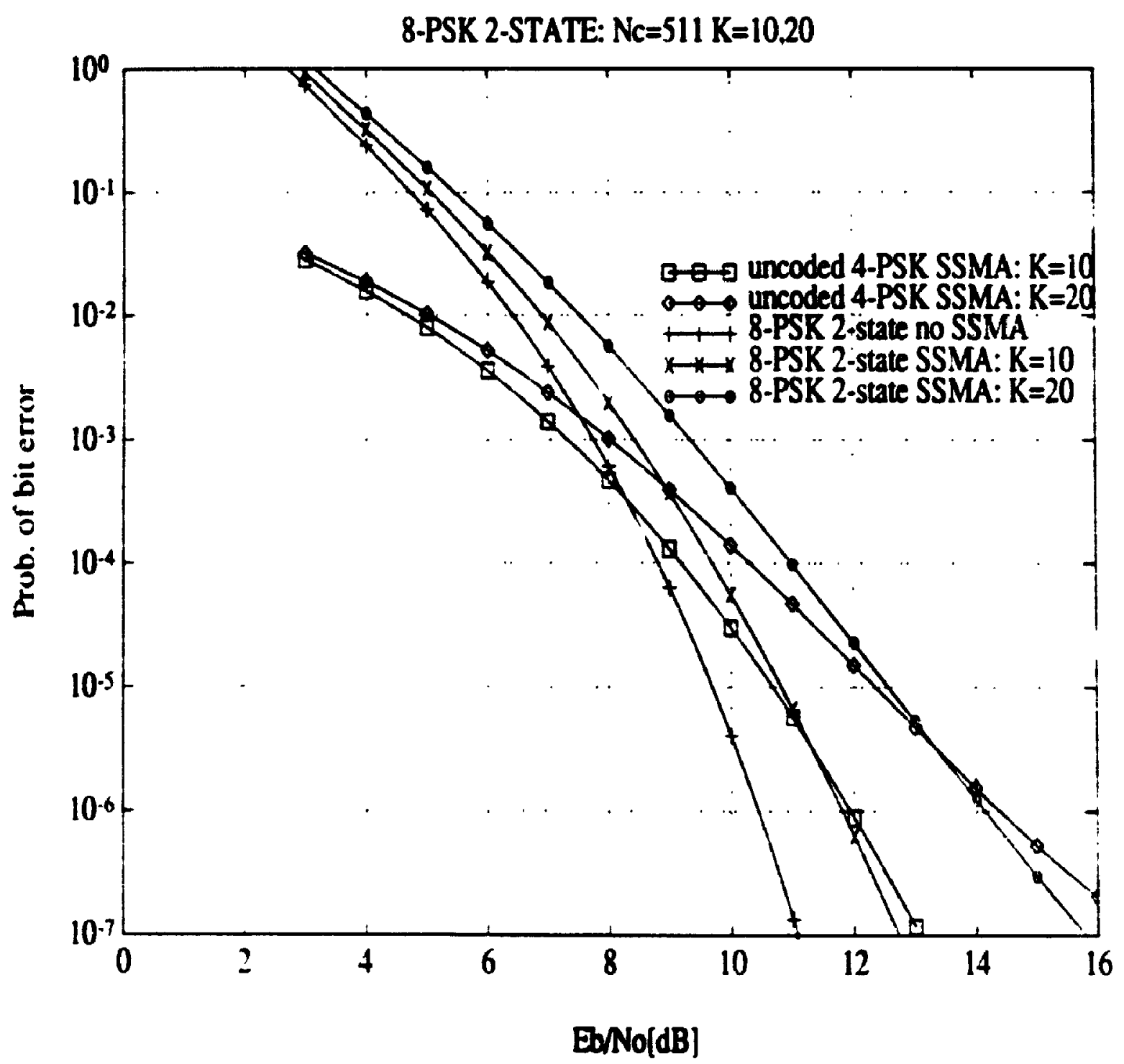

Figure 5.10: Performance of 8-PSK 2-state trellis codes for $k=10$ and $k=20$ users $\left(X_{c}=5(1)\right.$. 


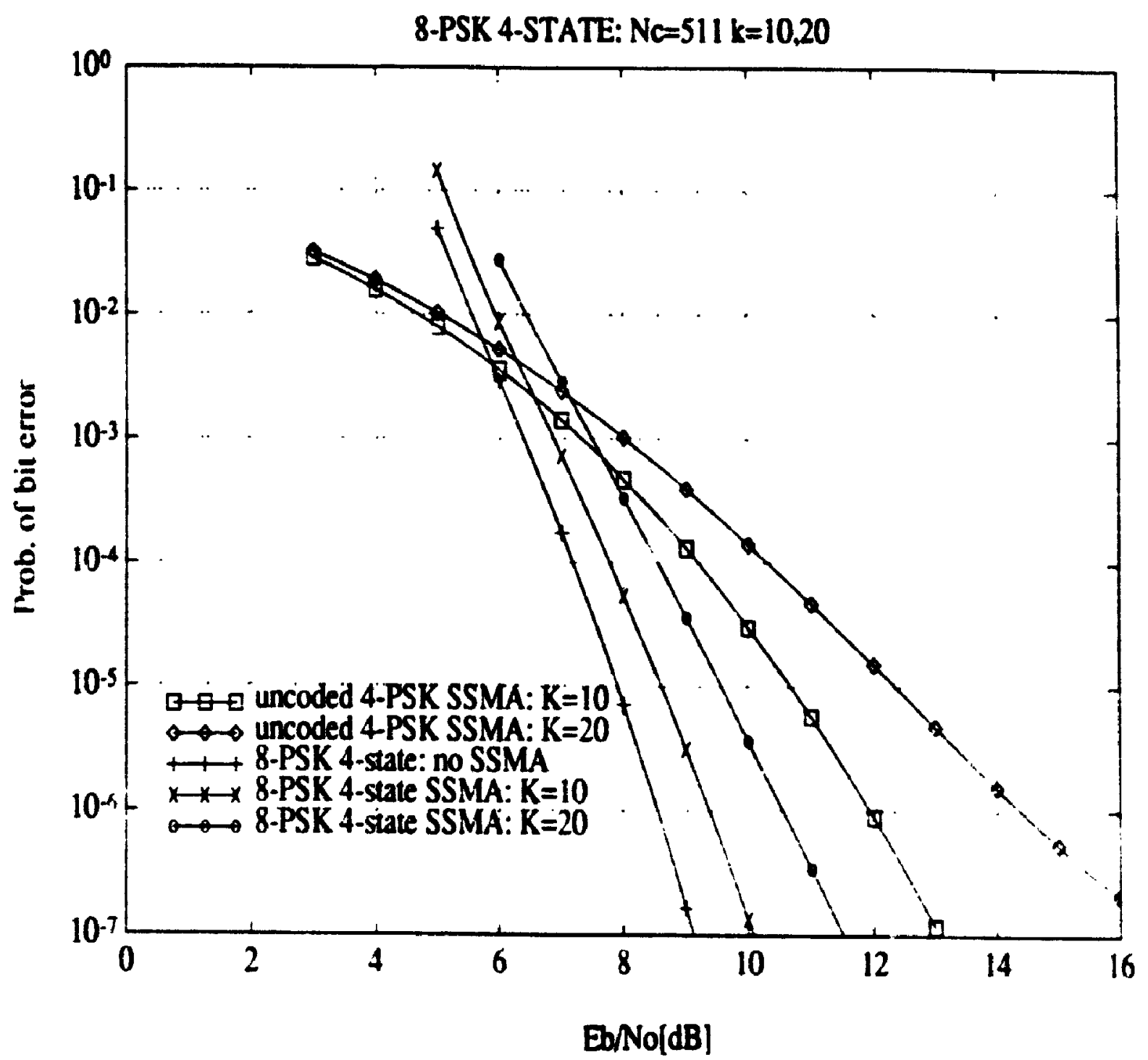

Figure 5.11: Performance of 8-PSK 4-state trellis codes for $K=10$ and $k=20$ users $\left(N_{c}=511\right)$. 


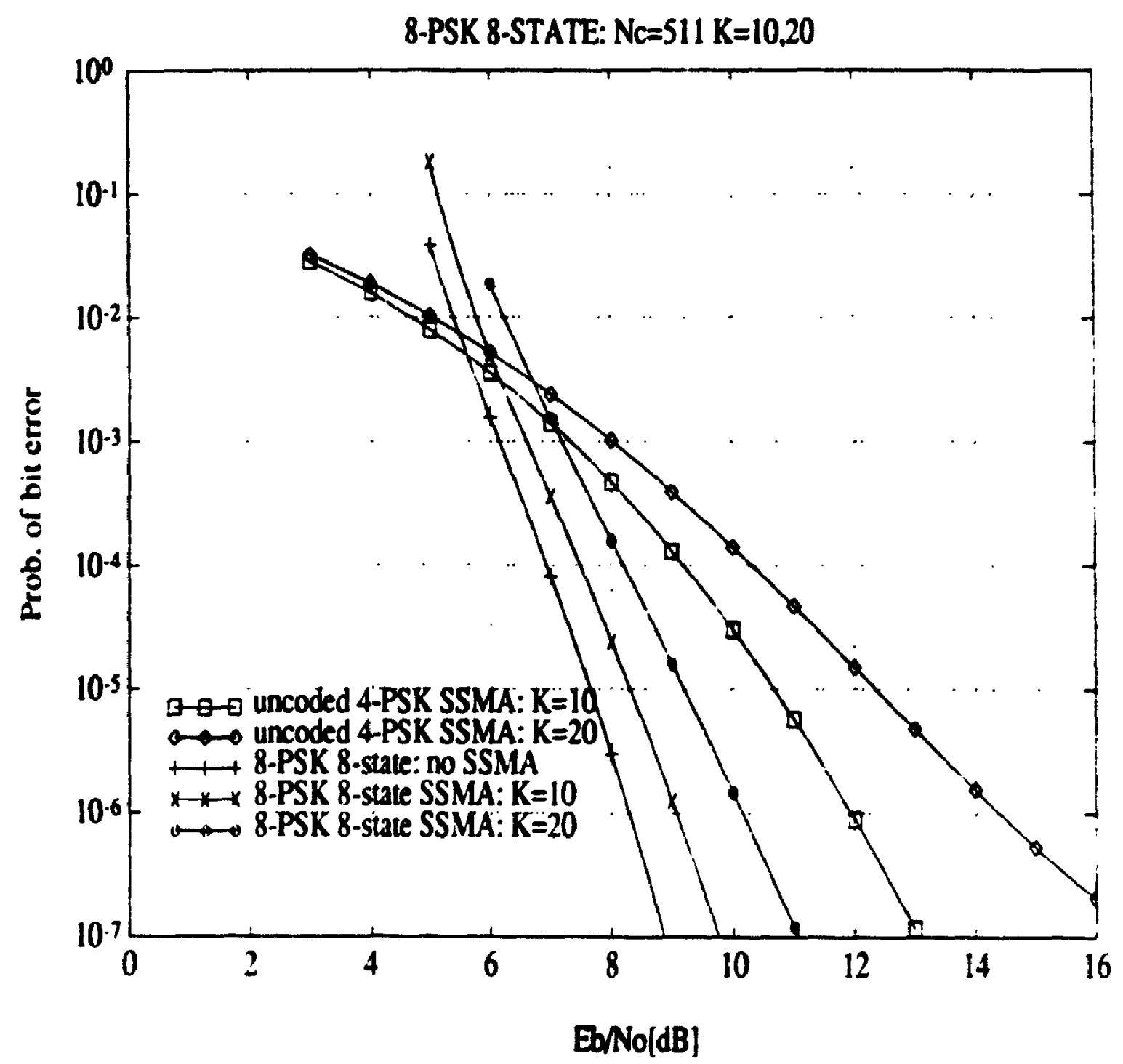

Figure 5.12: P'erformance of 8-PSK 8-state trellis codes for $K=10$ and $K=20$ users $(x=511)$. 


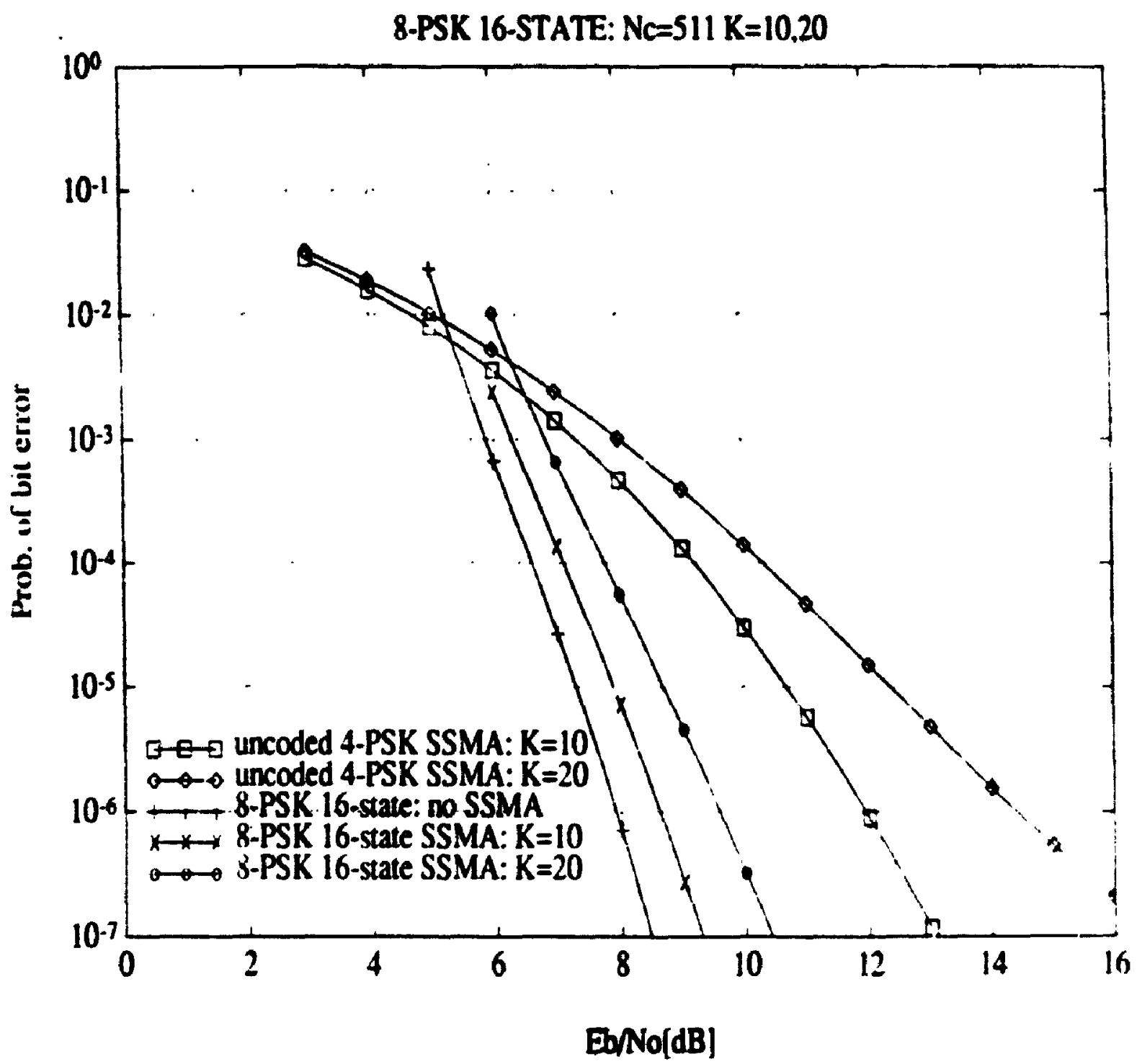

Figure 5.13: Performance of 8-PSK 16-state trellis codes for $k=10$ and $k=20$ users $\left(V_{\varepsilon}=511\right)$. 
rate implies that the 8-PSK code has a gain of at least 1.7dB over ihe 4-PSK code. A similar comparison for the 8 state codes shows that the 8 -PSK code has a gain of $0.5 \mathrm{~dB}$ over the 4-PSK code. A point of note is that the 8 -PSK rate $2 / 3$ codes degrade more quickly as the number of users is increased, than do the 4-PSK codes.

\subsubsection{6-PSK Codes}

Figures 5.14, 5.15 and 5.16 illustrate the performance of rate $\frac{3}{4}$ trellis codes, employing a 16-PSh signal constellation and having 4, 8 and 16 states respectively. The rncoders for these t rellis codes can be found in Figures 3.2, 3.4 and 3.6 respectively. ['erformance curves are ploted for the coded system with no IUI: $h=10$ multiple access users: and $K=20$ multiple access users. Also plotted for benchmark purposes are the performance curves of uncoded 8-PSK SSMA systems having $K=10$ and $K=20$ users respectively: Gold sequences of length $. V_{c}=511$ chips spanning one symbol period were employed as $P . N$ spreading sequences. For $K=10$ multiple access users. a probability of bit error rate of $10^{-5}$ is obtained with an $\frac{E_{8}}{. V_{0}}$ of $14 \mathrm{~dB}$ for the 1 state code, and $13.5 \mathrm{~d} B$ for the 8 state code. The coded curve for $K=20$ users saturates at a BER between $10^{-3}$ and $10^{-4}$. Using equal throughput as a basis of comparison. it can casily be determined that the 16-PSK codes are inferior to the S-PSK codes in BER performance. For example, at a BER of $10^{-5}$ the 16-PSK 1 stato code is 2.RdB inforior in BER performance than the 8-PSK 4 state code. even after accounting for the $1.77 \mathrm{~dB}$ degradation in the 8 -PSK code due to an increased noise bandwidth (by a factor of 1.5) in order to transmit at the no rate as the lij-P'Sh code. It should also be noted that the 16-PSK trellis codes are also inferior in performance than the 4 -PSK trellis codes on a basis of equal through it. 


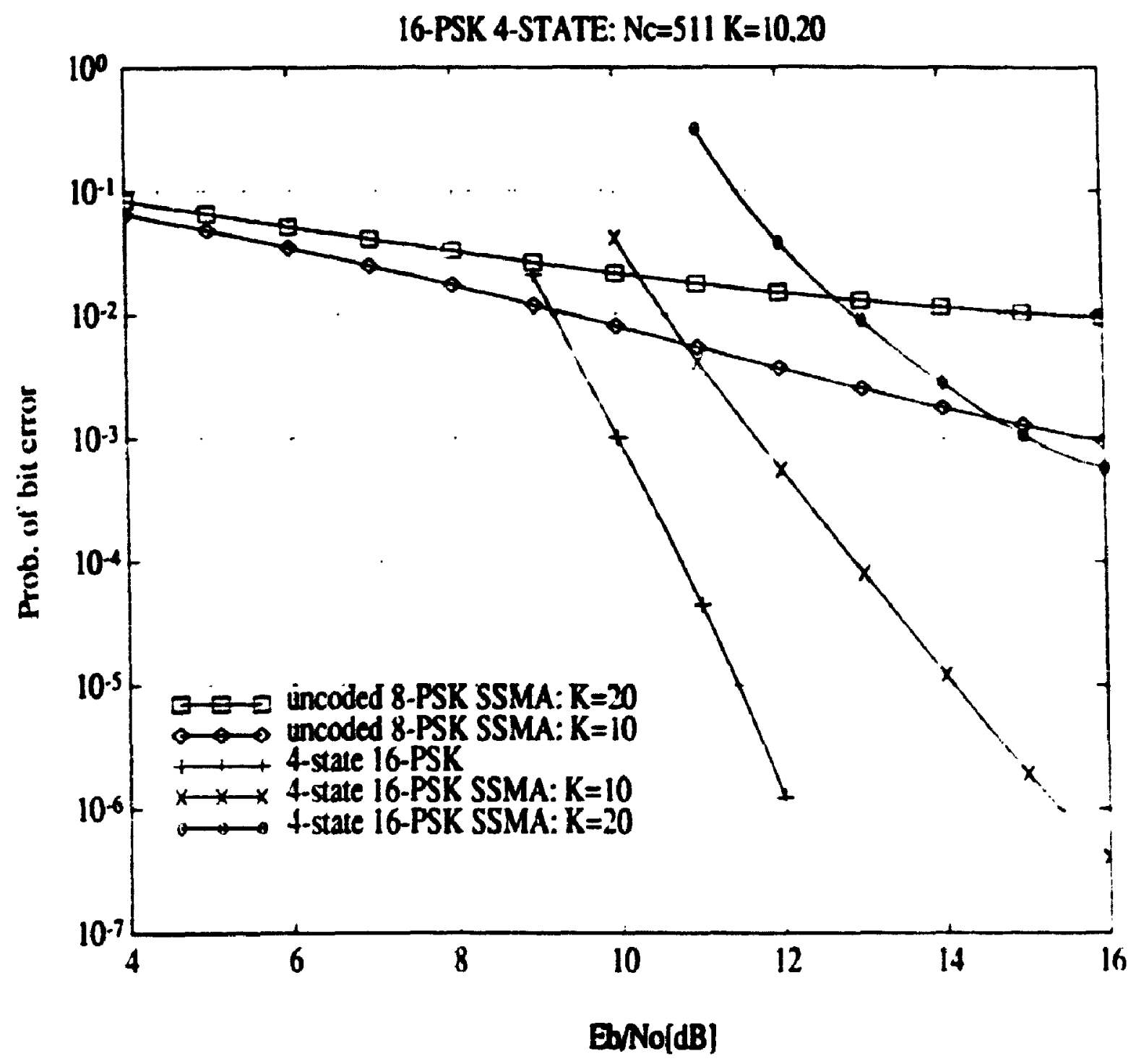

Figure 5.14: Performance of 16-PSK 4-state trellis codes for $k=10$ and $k=20$ users $\left(N_{c}=511\right)$. 


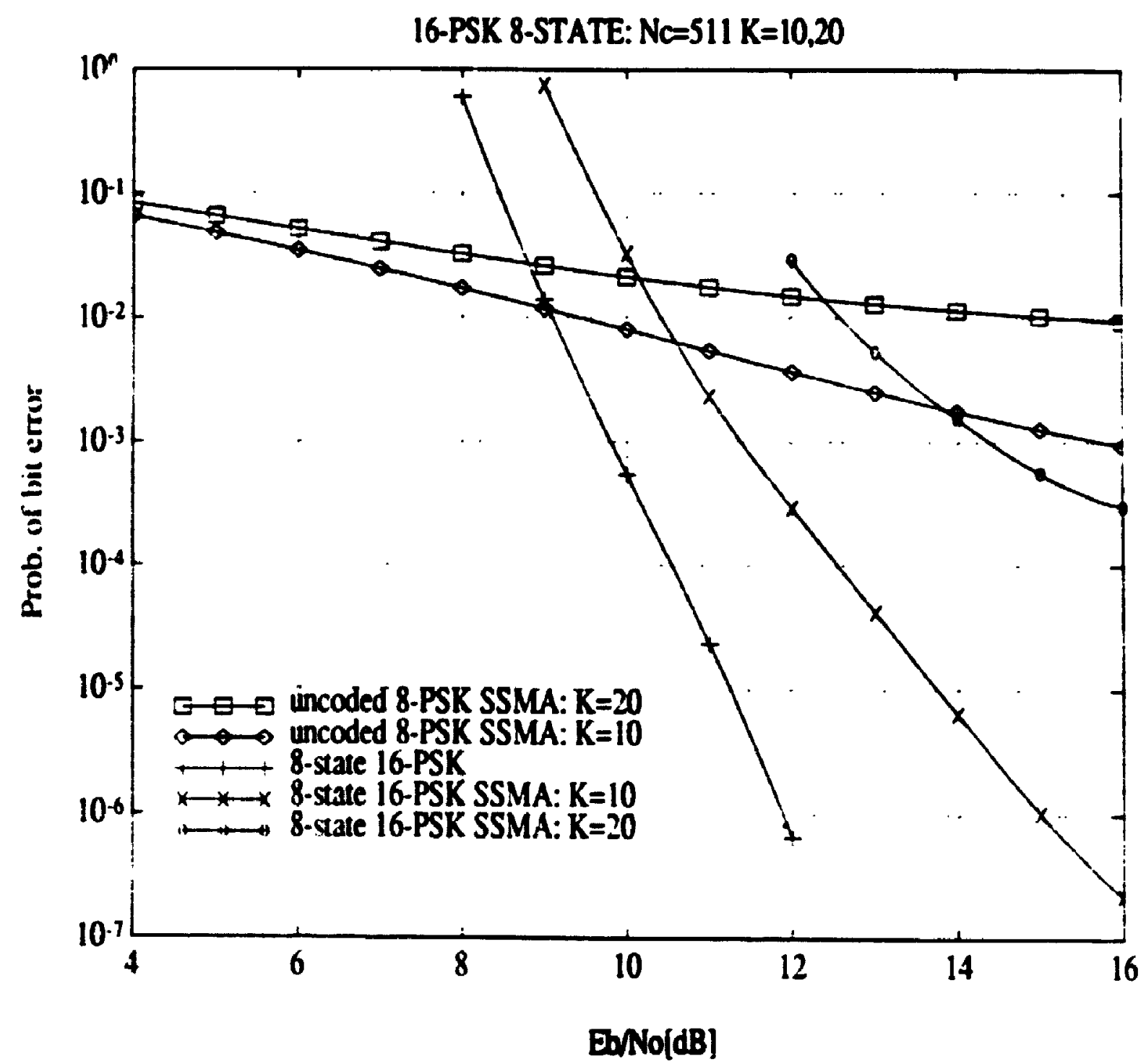

Figure 5.15: Performance of 16.PSK 8-state trellis codes for $K=10$ and $K=20$ users $\left(. x_{i}=511\right)$. 


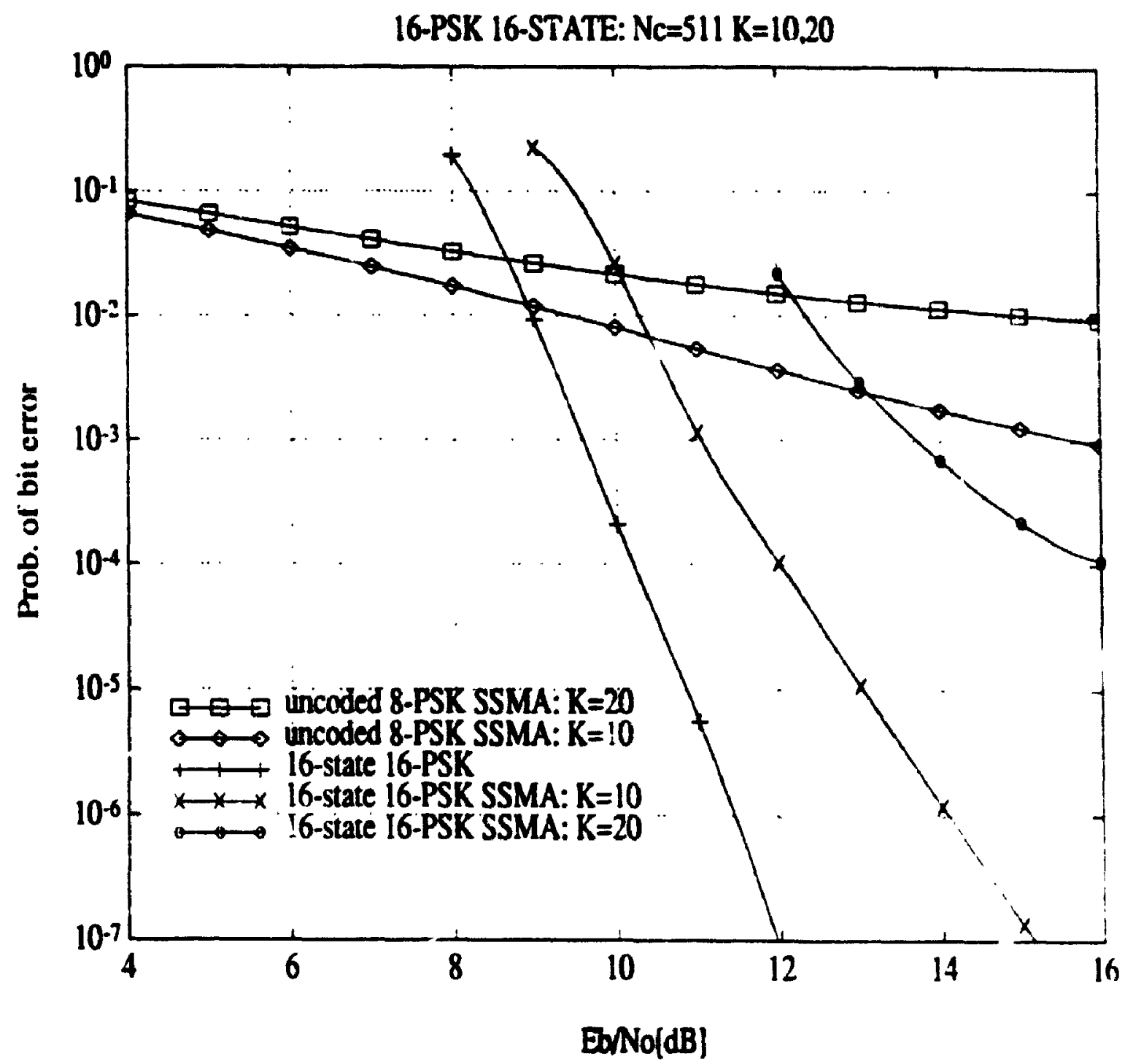

Figure 5.16: Performance of 16-PSK 16-state trellis codes for $K=10$ and $K=20$ users $\left(N_{c}=511\right)$. 


\subsection{Convolutionally coded SSMA}

The following results for convolutionally coded SSMA assume that the PN spreading sequence spans one coded symbol. As was explained in Chapter 2 and the introduction to Section 5 of this chapter, this implies that under the assumptions of fixed througiput (i.e. the data rate is constant), fixed maximum chip rate $\left(T_{c} \leq T_{c}^{\prime}\right)$ and fixed complexity, that a rate $\mathrm{k} / \mathrm{n}$ code must use a $\mathrm{PN}$ spreading sequence shorter by a factor of $1 / \mathrm{n}$ than that being employed by the trellis code to which it is being compared. This results in increased interuser interference due to the poorer cross correlation properties of shorter PN sequences. An alternative implementation of a convolutionally coded SSMA system would be to have the PN spreading sequence span more than one coded symbol. As was explained previously such an implementation results in poorer cross correlation properties since partial cross correlations between sequences must be accounted for, which are inherently more severe than cross correlations spanning a full sequence[20].

\subsubsection{Rate $1 / 2$ Codes}

Figures 5.17, 5.18 and 5.19 illustrate the perfor $r$ ance of rate $\frac{1}{2}$ convolutional codes. employing a BPSK signal constellation and having 4, 8 and 16 states. respectiveiy. The encoders for these convolutional codes are described by the generator polynomials given in the first three rows of Table 3.7. Since these convolutional codes are being compared to trellis codes using Gold spreading sequences of length $N_{c}=511$, the performance of these rate $1 / 2$ convolutionally coded SSMA systems was evaluated using Gold spreading sequences of half this length (i.e. $N_{c}=255$ ). Though in fact this is not exactly half of the sequence length of the trellis codes, the resulting difference in the performance curves are negligible and can easily be compensated for by appropriately scaling the symbol to noise ratio. Performance curves are plotted for a convolutionaily coded SSMA system having no IUI (i.c. just $\dot{A} W G N$ ); $K=$ 


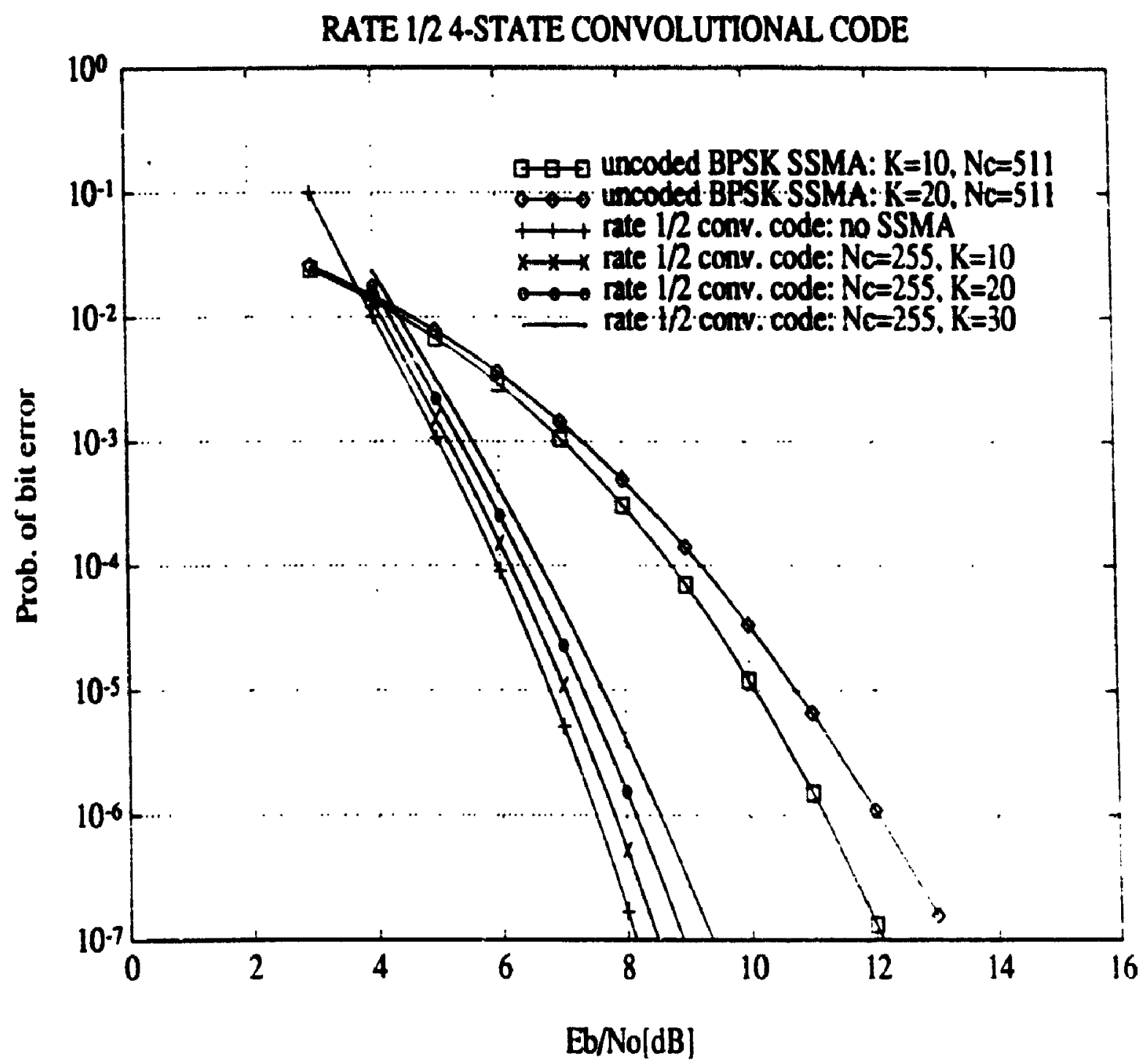

Figure 5.17: Performance of rate 1/2 4-state convolutional codes for $K=10, K=20$ and $\mathrm{K}=30$ users $\left(N_{c}=255\right)$. 


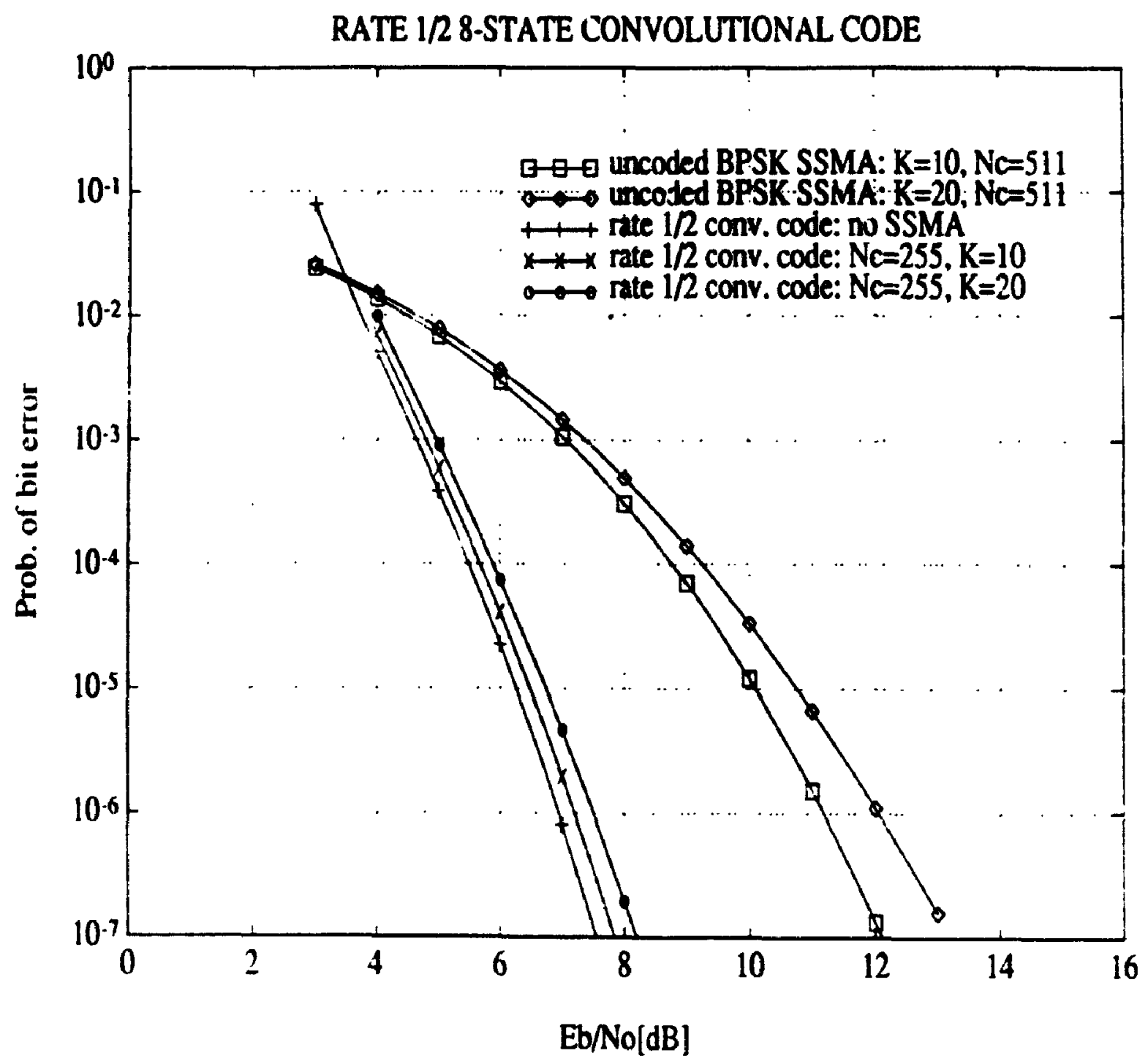

Figure 5.18: Performance of rate $1 / 28$-state convolutional codes for $K=10$, and K $=20$ users $\left(N_{i}=255\right)$. 


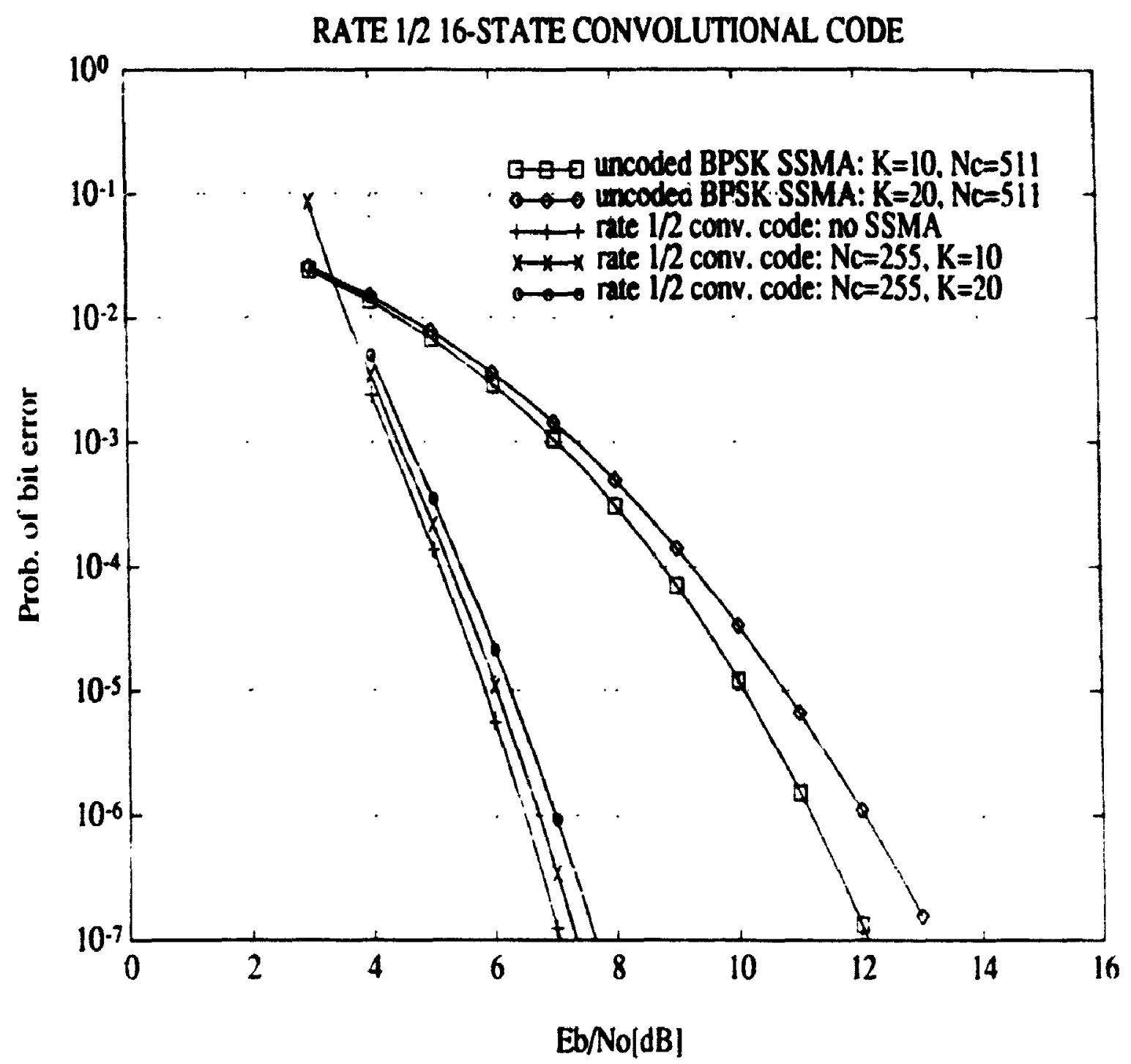

Figure 5.19: Performance of rate $1 / 216$-state convolutional codes for $K=10$. and $\mathrm{K}=20$ users $\left(N_{c}=255\right)$. 
10 multiple access users; $K=20$ multiple access users; and $K=30$ multiple access users. Also plotted for benchmark purposes are the performance curves of uncoded BPSK SSMA systems having $K=10$ and $K=20$ users respectively. For a BER of $10^{-5}$ and $\mathrm{K}=20$ users it can be seen that the convolutionally coded systems exhibit a gain of $3.4 \mathrm{~dB}$ for the 4 state code, $4.1 \mathrm{~dB}$ for the 8 state code and $4.6 \mathrm{~dB}$ for the 16 state code. Similar gains are illustrated for $K=10$ users. From the above figures it can also be scen that each additional 10 multiple access users causes an additional degradation of less than $0.5 \mathrm{~dB}$.

Comparing the performance of the rate $1 / 2$ convolutional codes to the rate $1 / 2$ 4-PSK trellis codes (i.e., these are the trellis codes having tl.e same throughput as the rate $1 / 2$ convolutional codes), it can be seen that the rate $1 / 2$ convolutional codes provide marginally better (i.e. $0.1 \mathrm{~dB}$ to $0.2 \mathrm{~dB}$ ) BER performance than do the rate $1 / 2$ trellis codes.

\subsubsection{Rate 1/8 Codes}

Figures 5.20, 5.21 and 5.22 illustrate the performance of rate $\frac{1}{8}$ convolutional codes, employing a BPSK signal constellation and having 4.8 and 16 states. respectively. The encoders for these convolutional codes are described by the generator polynomials given in Table 3. $\pi$. Since these convolutional codes are being compared to trellis rociss using (iold spreading sequences of length $N_{c}=511$, the performance of these rate $1 / 8$ convolutionally coded SSMA systems was evaluated using Gold spreading sequences of $1 / 8$ this length (i.e. $N_{c}=63$ ). Though in fact this is not exactly $1 / 8$ of 511 as before. the resulting difference in the performance curves are negligible and can easily be compensated for by appropriately scaling the symbol to noise ratio. Performance curves are plotted for a convolutionally coded SS.MA system having no III (i.e. just $A W G N$ ); $K=10$ multiple access users: and $K=20$ multiple access users. Also plotted for benchmark purposes are the performance curves of uncoded BPSK SSMA systems having $K=10$ and $K=20$ users respectively. For a BER of 


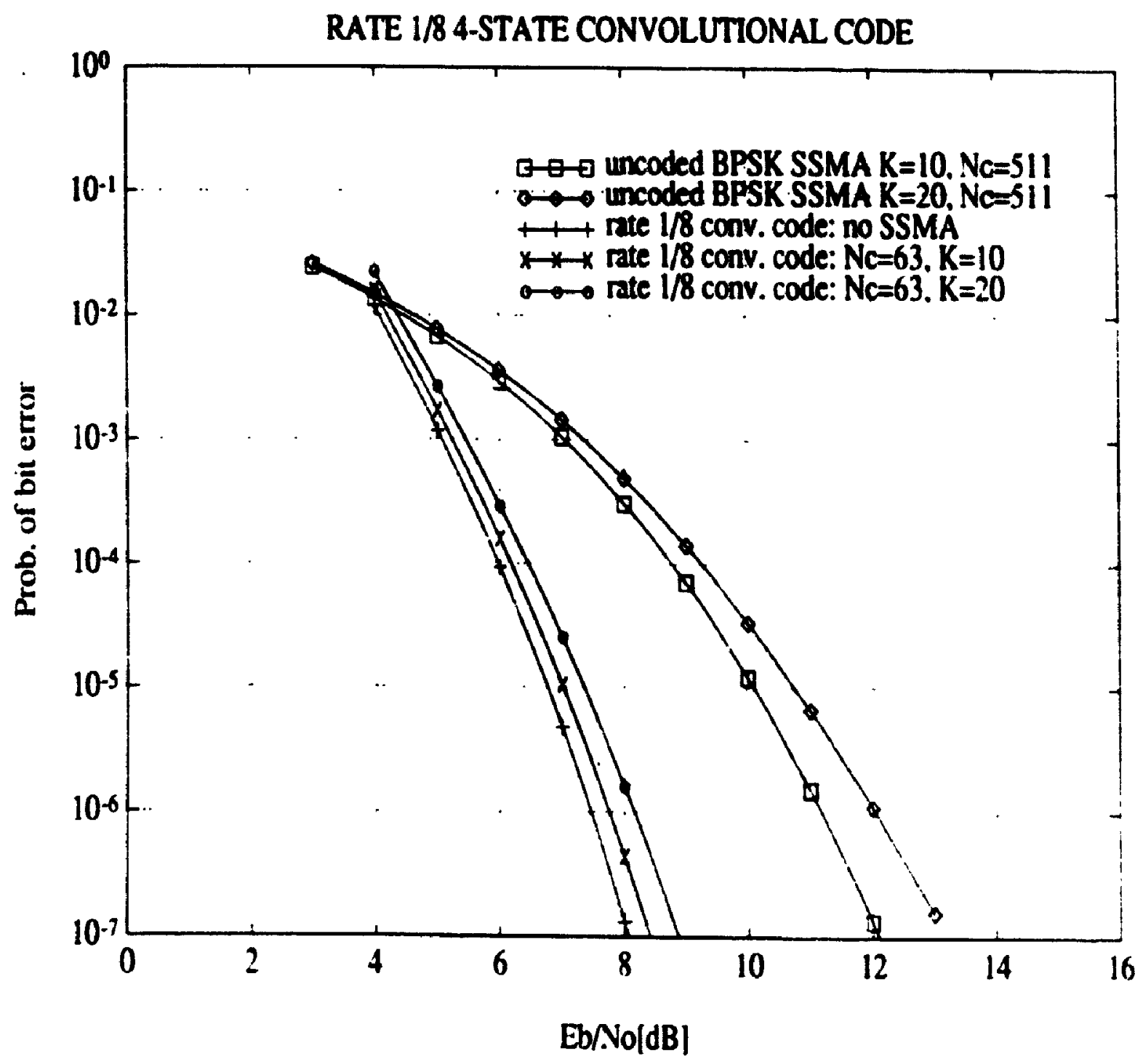

Figure 5.20: Performance of rate 1/8 4-state convolutional codes for $K=10$ and $K=20$ ) users $\left(N_{c}=63\right)$. 


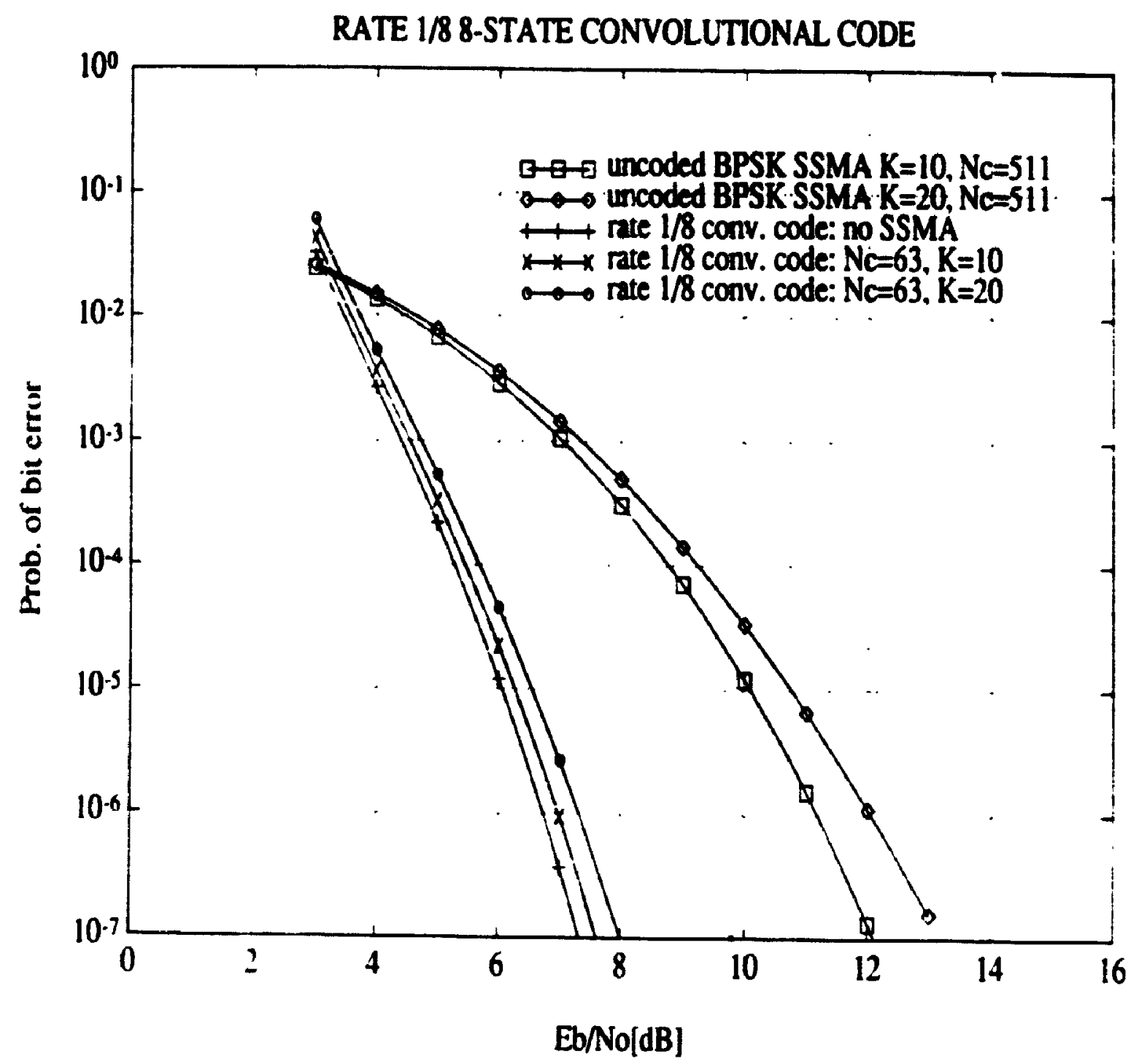

Figure 5.21: Performance of rate 1/8 8-state convolutional codes for $\mathrm{K}=10$ and $\mathrm{K}=20$ users $\left(I_{c}=63\right)$. 


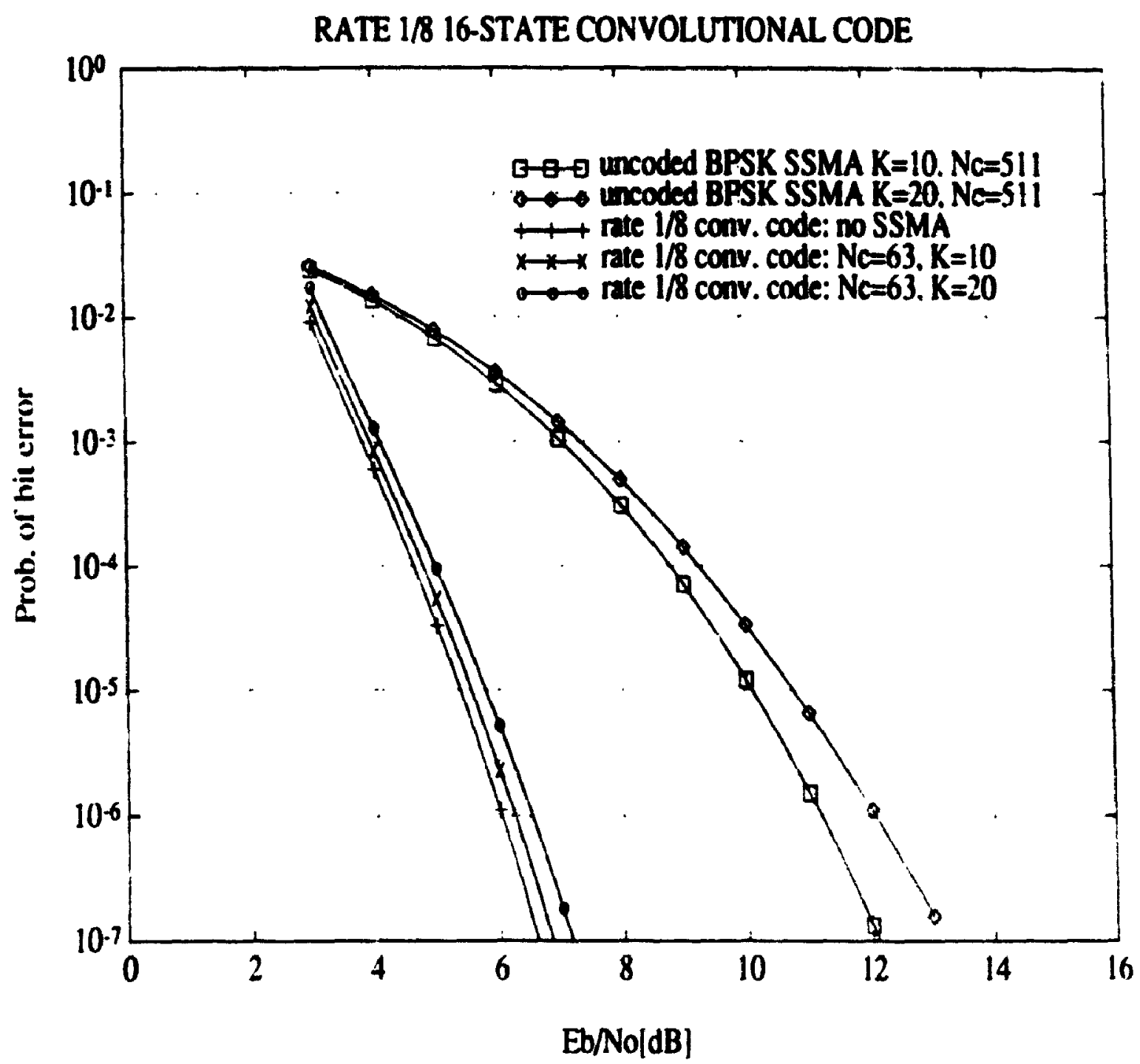

Figure 5.22: Performance of rate $1 / 8$ 16-state convolutional codes for $K=10$ and $\mathrm{K}=20$ users $\left(N_{c}=63\right)$. 
$10^{-5}$ and $K=20$ users it can be seen that the convolutionally coded systems exhibit a gain of $3.4 \mathrm{~dB}$ for the 4 state code, $4.2 \mathrm{~dB}$ for the 8 state code and $5.0 \mathrm{~dB}$ for the 16 state code. Similar gains are illustrated for $K=10$ users.

Comparing the performance of the rate $1 / 8$ convolutional codes to the rate $1 / 2$ 4-PSK trellis codes and the rate $1 / 2$ convolutional codes, it can be seen that the rate $1 / 8$ convolutional codes provide marginally better (i.e. $0.3 \mathrm{~dB}$ to $U .5 \mathrm{~dB}$ ) BER performance than do the rate $1 / 2$ convolutional codes, with a corresponding increase over the rate 1/2 1-PSK trellis codes. This gain in performance is more pronounced for the more complex codes (i.e. the 16 state code).

\subsubsection{Rate 2/3 Codes}

Figures 5.23, and 5.24 illustrate the performance of rate $\frac{2}{3}$ convolutional codes, employing a BPSh signal constellation and having 4 and 16 states, respectively, with generator polynomials as defined in Table 3.7. The PN sequences employed fur DS spreading of the information symbols were Gold codes of length $N_{c}=127$. These convolutional codes transmit two information bits per 3 coded symbols. As such they can be compared to the rate 2/3 8-PSK trellis codes on an equitable basis, since each PN sequence spans one coded symbol. Performance curves are plotted for a convolutionally coded SSMA system having no IUI (i.e. just AWGN); $k=10$ multiple access users: and $K=20$ multiple access users. Also plotted for benchmark purposes are the performance curves of uncoded 4-PSK SSMA systems having $K=$ 10 and $K=20$ users respectively. For a BER of $10^{-5}$ and $K=20$ users it can be seen that the convolutionally coded systems exhibit a gain of $2.5 \mathrm{~dB}$ for the 4 state code, and $4.6 \mathrm{~dB}$ for the 16 state code, over the uncoded schemes. Similar gains are illustrated for $k=10$ users.

Comparing the performance of the rate $2 / 3$ convolutional codes to the rate $2 / 3$ 8-PSK trellis codes of Figures 5.11 and 5.13 (i.e. these are the trellis codes having the same throughput as the rate $2 / 3$ convolutional codes), it can be seen that at a BER 


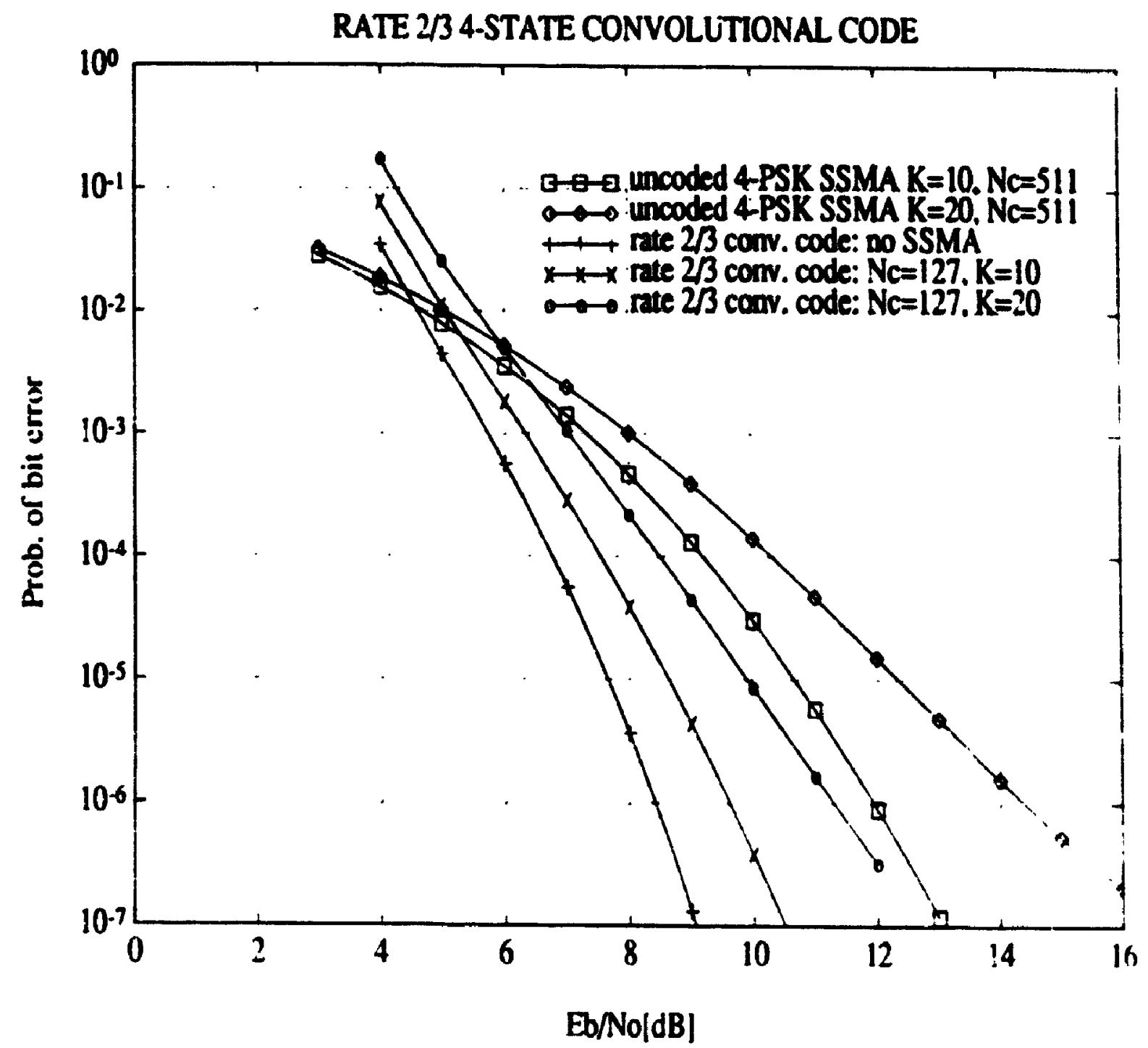

Figure 5.23: Performance of rate $2 / 3$ 4-state convolutional codes for $K=10$ and $K=20$ users $\left(N_{c}=127\right)$. 


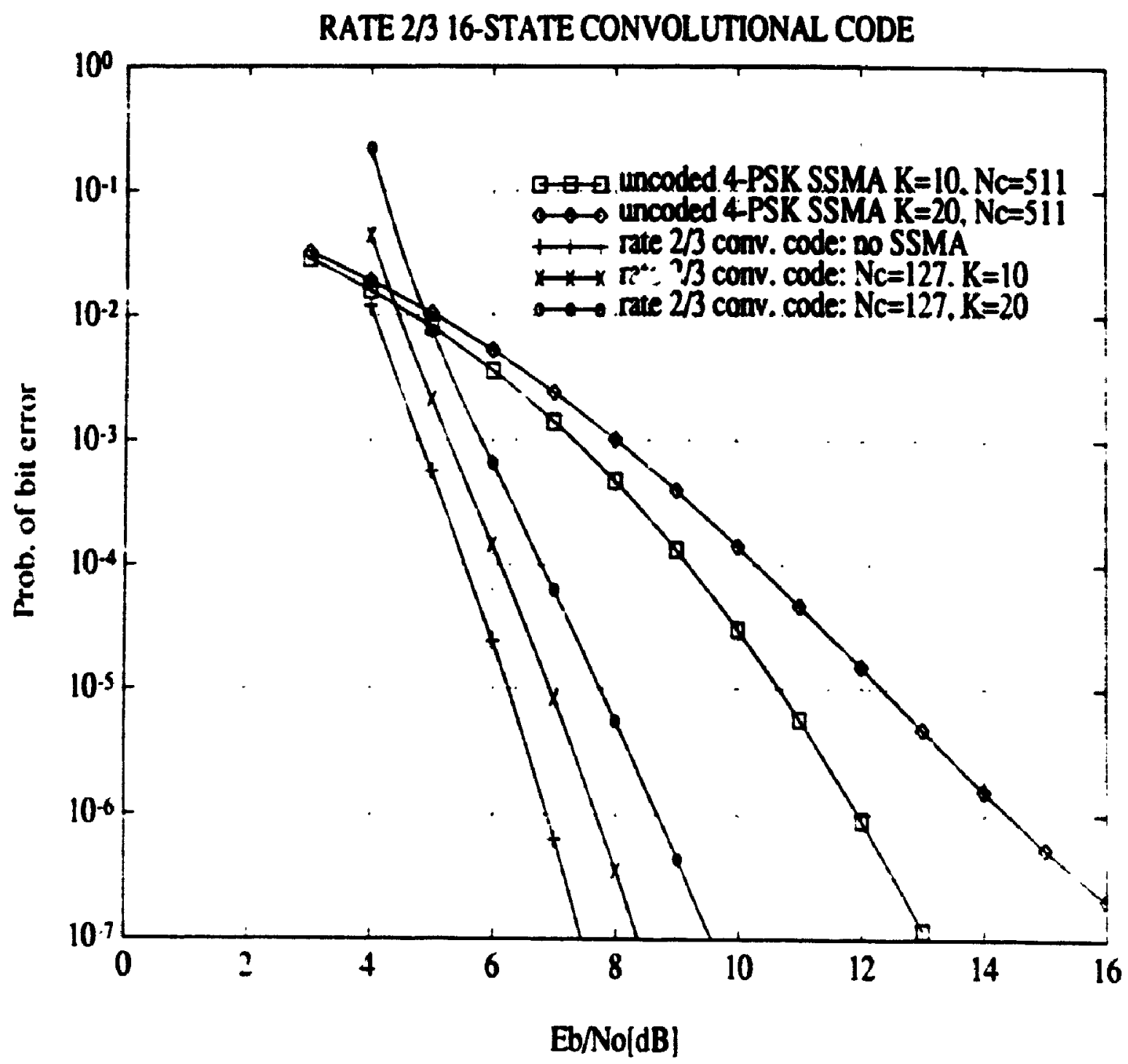

Figure 5.24: Performance of rate 2/3 16-state convolutional codes for $\mathrm{K}=10$ and $h=20$ users $\left(N_{r}=127\right)$. 
of $10^{-5}$ and $K=20$ users that the 4 state rate $2 / 3$ convolutional code is about $0.3 \mathrm{~dB}$ inferior to the corresponding rate 2/3 8-PSK trellis code. However, the 16 state rate 2/3 convolutional code is approximately $1 \mathrm{~dB}$ superior in performance to the 16 state rate $2 / 3$ trellis code at a BER of $10^{-5}$ for both $k=10$ and $k=20$ users. Thus as in the case of the rate $1 / 2$ codes. as codes with higher complexity are employed, the convolutional codes tend to outperform the corresponding trellis codes.

\subsubsection{Rate 2/7 Codes}

Figures 5.25, and 5.26 illustrate the performance of rate $\frac{2}{7}$ convolutional codes. employing a BPSK signal constellation and having 4 and 16 states. respectively. with generator polynomials as defined in Table 3.T. The PN sequences employed for DS spreading of the information symbols were Gold codes of length $\Lambda_{c}=63$. As in the case of the rate $2 / 3$ convolutional codes, these convolutional codes transmit two information bits per $\boldsymbol{i}$ coded symbols, and as such they can be compared to the rate 2/3 8-PSK trellis codes on an equitable basis, since cach PN sequence spans one coded symbol. Performance curves are plotted for a convolutionally coded SSMA system having no IUI (i.e. just $A W G N$ ); $k=10$ multiple access users: and $k=$ 20 multiple access users. Also plotted for benchmark purposes are the performance curves of uncoded 4-PSK SSMA systems having $k=10$ and $k=20$ users resper. tively. For a $B E R$ of $10^{-5}$ and $k=20$ users it can be seen that the convolutionally coded systems exhibit a zain of $4.1 \mathrm{~dB}$ for the 4 state code. and $5.9 \mathrm{~dB}$ for the 10 state code. over the uncoded schemes. Similar gains are illustrated for $k=10$ users.

Compasing the performance of the rate $2 / 7$ convolutional codes to the rate $2 / 3$ 8-PSK trellis codes of Figures 5.11 and 5.13 it can be seen that at a BER of $10^{-1}$ and $K=20$ users that the 4 state rate $2 / 7$ convolutional code is about $1.3 \mathrm{~dB}$ su;rrior to the corresponding rate $2 / 3$ 8-PSK trellis code. Furthermore. th 16 st. . e rate $2 / i$ convolutional code is approximately $2.3 \mathrm{~dB}$ superior in performance to t: 16 state 


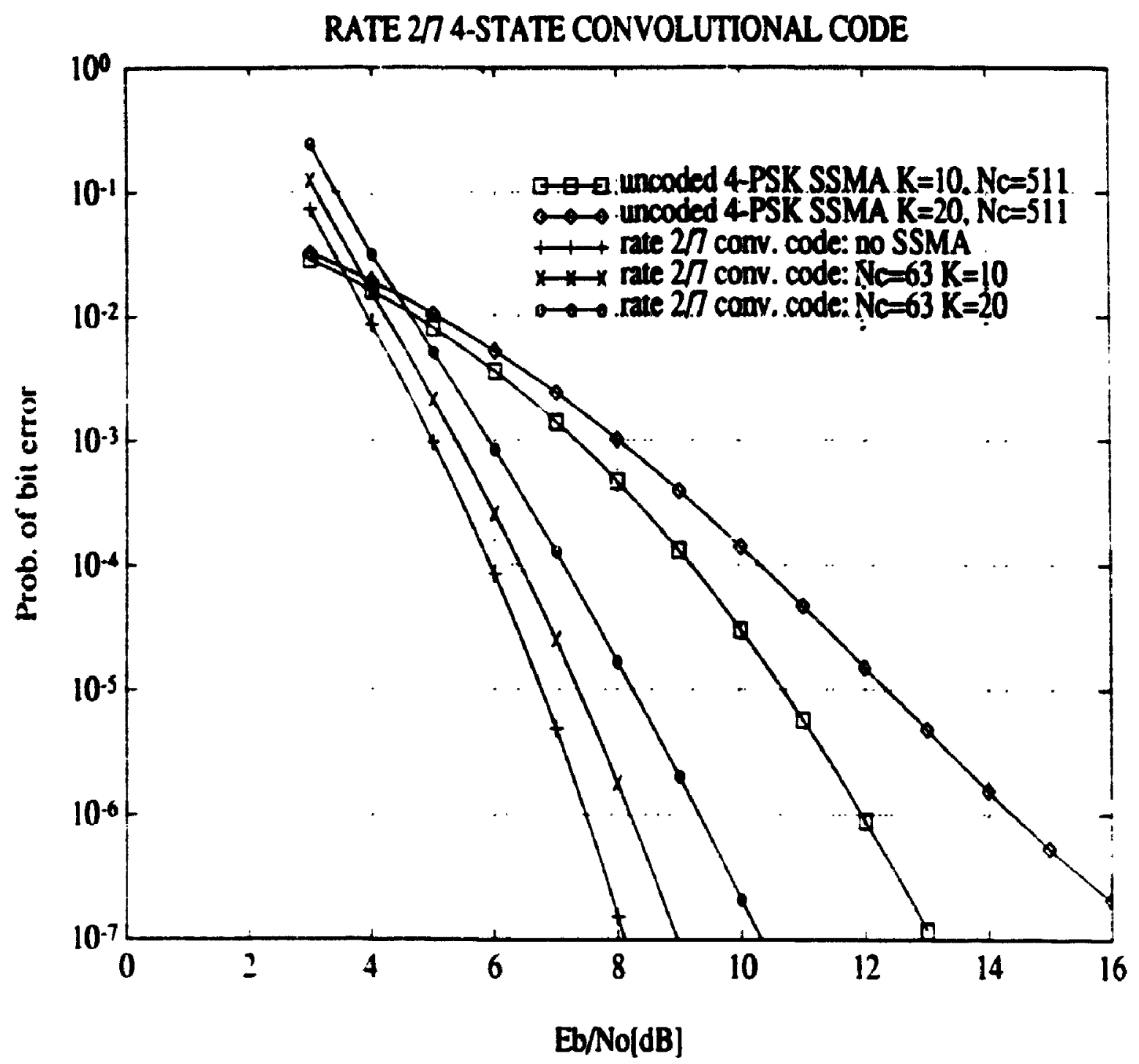

Figure 5.25: Performance of rate 2/7 4-state convolutional codes for $K=10$ and $K=20$ users $\left(. V_{c}=63\right)$. 


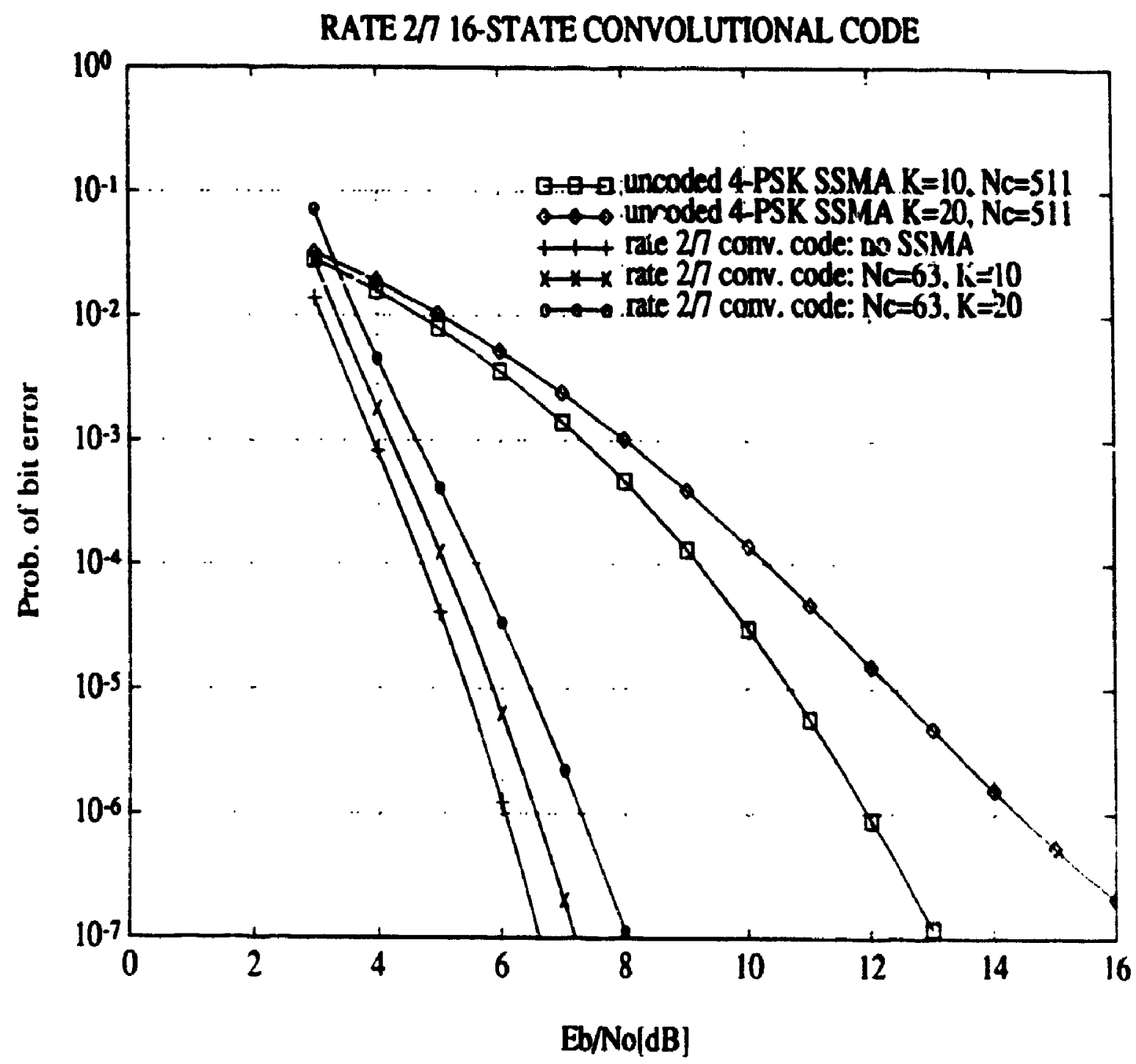

Figure 5.26: Performance of rate 2/7 16-state convolutional codes for $k=10$ and $\mathrm{K}=20$ users $\left(N_{c}=63\right)$. 
rate $2 / 3$ 8.PSK trellis code at a $B E R$ of $10^{-5}$ for both $K=10$ and $K=20$ users. It should also be noted that the rate $2 / 7$ convolutional codes are superior to the rate $2 / 3$ convolutional codes and on a basis of equal throughput. are superior to the rate $1 / 8$ convolutional codes.

Two treids are exhitited in the above results. As lower rate convolutional codes are employed, one obtains superior performance over both medium rate convolutional codes and over the corresponding trellis codes, at a slight increase in complexity, and furthermore, this increase in coding gain becomes more pronounced as the number of states is increased.

\subsection{Monte Carlo Simulations}

A Monte Carlo simulation of the performance of both trellis and convolutionally coded SSMA was carried out in order to benchmark and verify the accuracy of the Chernoff upper bound performance presented in the previous sections in this chapter. Figure 5.27 compares the simulated performance of the 4-state rate 1/2 4-PSK trellis code to the simulated performance of the 4 -state rate $1 / 2$ convolutional code. The trellis coded SSMA system is assumed to emplcy Gold spreading sequences of length $V_{.}=127$ chips. Whereas the rate $1 / 2$ convolutionally coded system is assumed to 'mploy Cold spreading sequences of length $N_{c}=63$ chips. Both systems assume the presence of $\mathrm{K}=5$ users. The Monte Carlo simulations points a- Jlotted with a second order least squares error polynomia! fit, and the error bars inaicate 95 percent confidence intervals on the abscissa. From these curves it can be that the true performance of the coded SSMA is approximately $1 \mathrm{~dB}$ superior than that indicated by the chernoff upper bound performance. The slight superiority of the rate $1 / 2$ convolutional code is ronsistent with the results obtained by the Chernoff bound. 


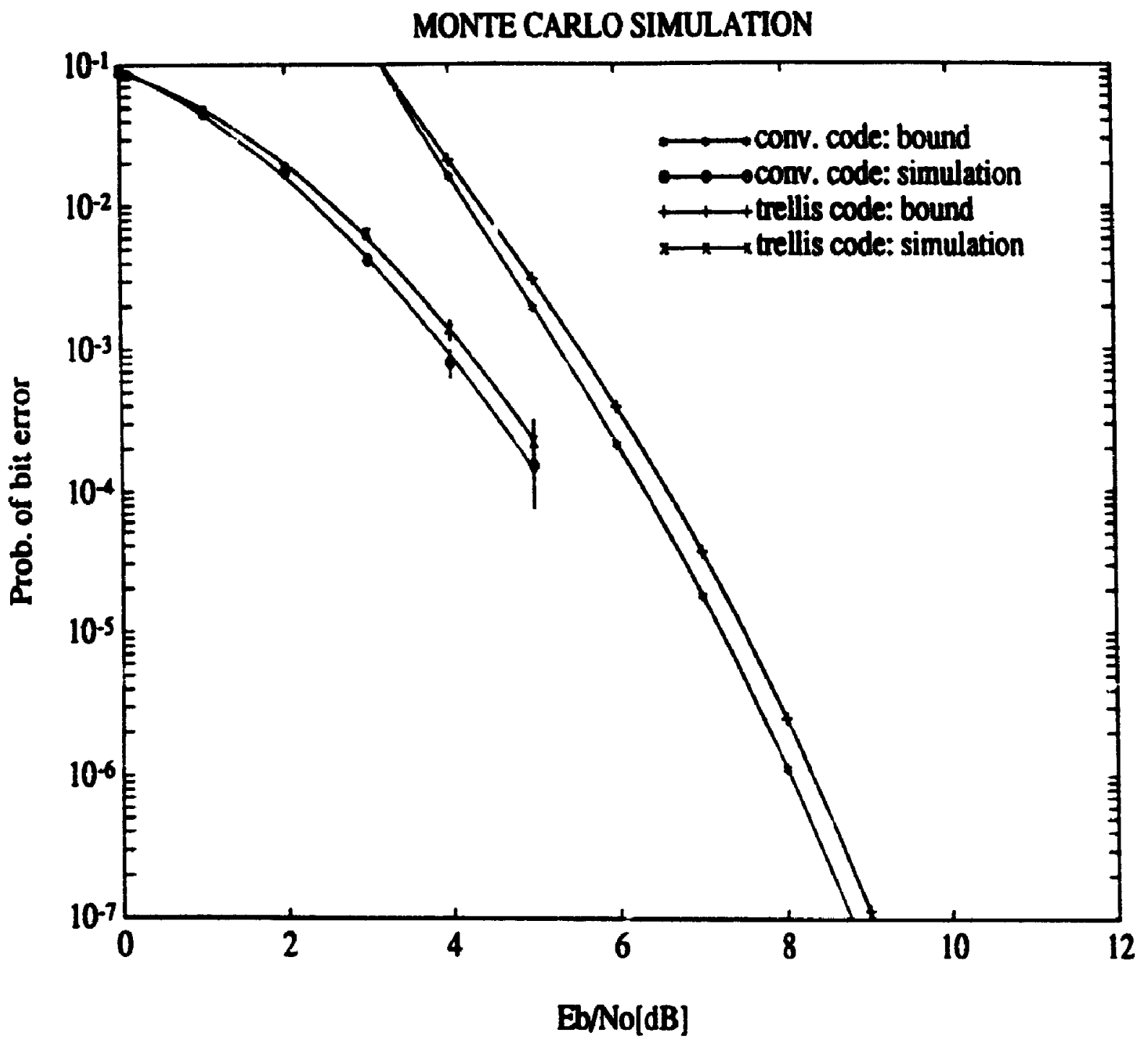

Figure 5.27: Performance of Monte Carlo simulation for rate 4-state rate 1/2 4PSK trellis code versus 4 -state rate $1 / 2$ convolutional code 


\subsection{Gaussian Approximation of the Interuser In- terference}

As was outlined in Chapter 4, Section 4.3 , in order to simplify the calculation of the performance of a SSMA system the IUI interference random variable (in this case $z_{p}$ ) is often assumed to be a Gaussian random variable. For uncoded SSMA systems, previous use of this approximation by other authors has found that the resulting predicted performance is overly optimistic as compared to more exact bounding techniques (such as the method of moments)[2][26].

Several of the above codes have been evaluated in an AWGN noise channel employing SSMA, and the IUI has been approximated using a Gaussian random variable. The results are presented in Figures 5.28, 5.29, 5.30, 5.31 and 5.32 for the 4-PSK 4-state, 8-PSK 4-state, 8-PSK 16-state trellis codes as well as rate 1/8 and $2 / 7$ 4-state convolutional codes, respectively. The results of these curves show that the use of the Gaussian approximation in coded SSMA systems also tends to underbound the predicted Chernoff upper bound performance. For the rate $1 / \mathrm{n}$ codes the Gaussian approximation is overly optimistic by roughly $0.25 \mathrm{~dB}$ for $\mathrm{K}=10$ users and $0.5 \mathrm{~dB}$ for $\mathrm{K}=20$ users. For the rate $2 / \mathrm{n}$ codes these numbers change to $0.4 \mathrm{~d} b$ and $0.9 \mathrm{~dB}$ for $K=10$ and $K=20$ users respectively. The two trends to note in the above resu'ts are that the degree to which the Gaussian approximation is overly optimistic increases with the throughput of the code as well as the number of users present in the SSMA system.

\subsection{Partial Correlation Results}

As was detailed in Chapter 2 and earlier in this Chapter, a second implementation of convolutionally coded 'SSMA systems involves having each coded symbol span a subseyuence of the complete PN sequence as opposed to having each coded symbol 


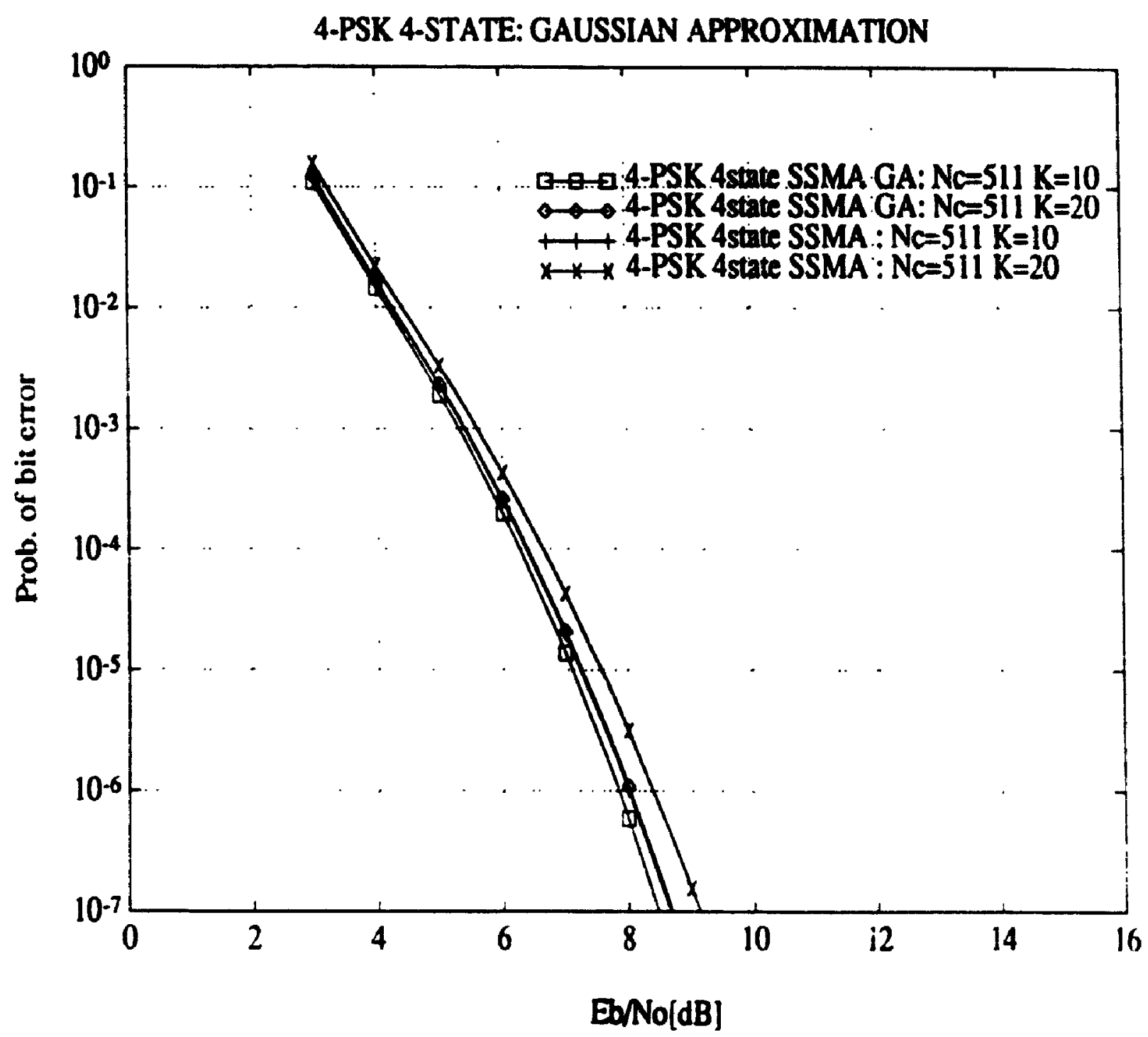

Figure 5.28: Performance of 4-PSK 4-state trellis code for $K=10$ and $K=20$ users employing the Gaussian Approximation $\left(N_{c}=511\right)$. 


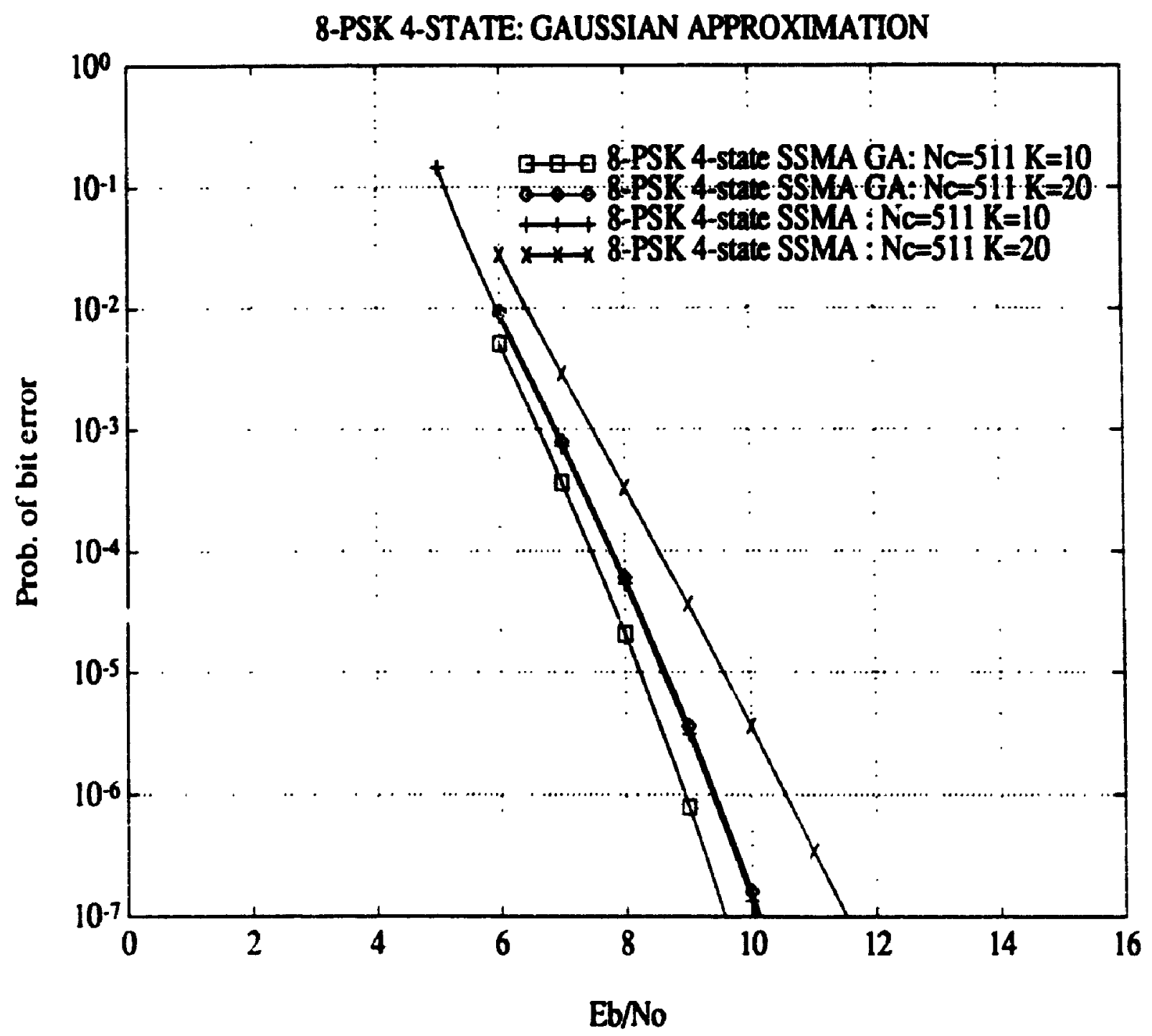

Figure 5.29: Perfornance of 8-PSK 4-state trellis code for $K=10$ and $K=20$ users (mploying the Ga'ssian Approximation $\left(N_{c}=511\right)$. 


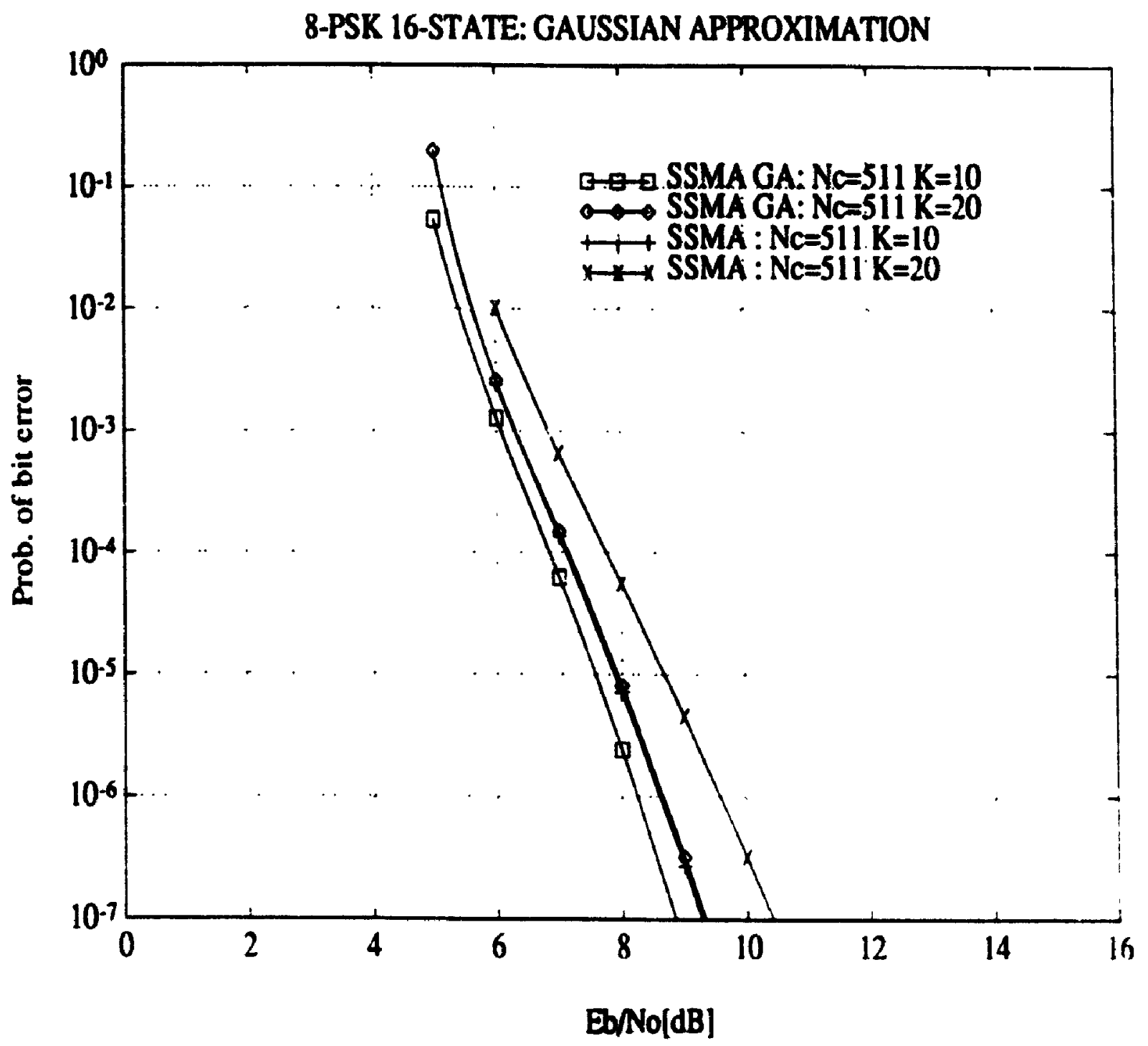

Figure 5.30: Performance of 8-PSK 16-state trellis code for $K=10$ and $K=20$ users employing the Gaussian Approximation $\left(N_{c}=511\right)$. 


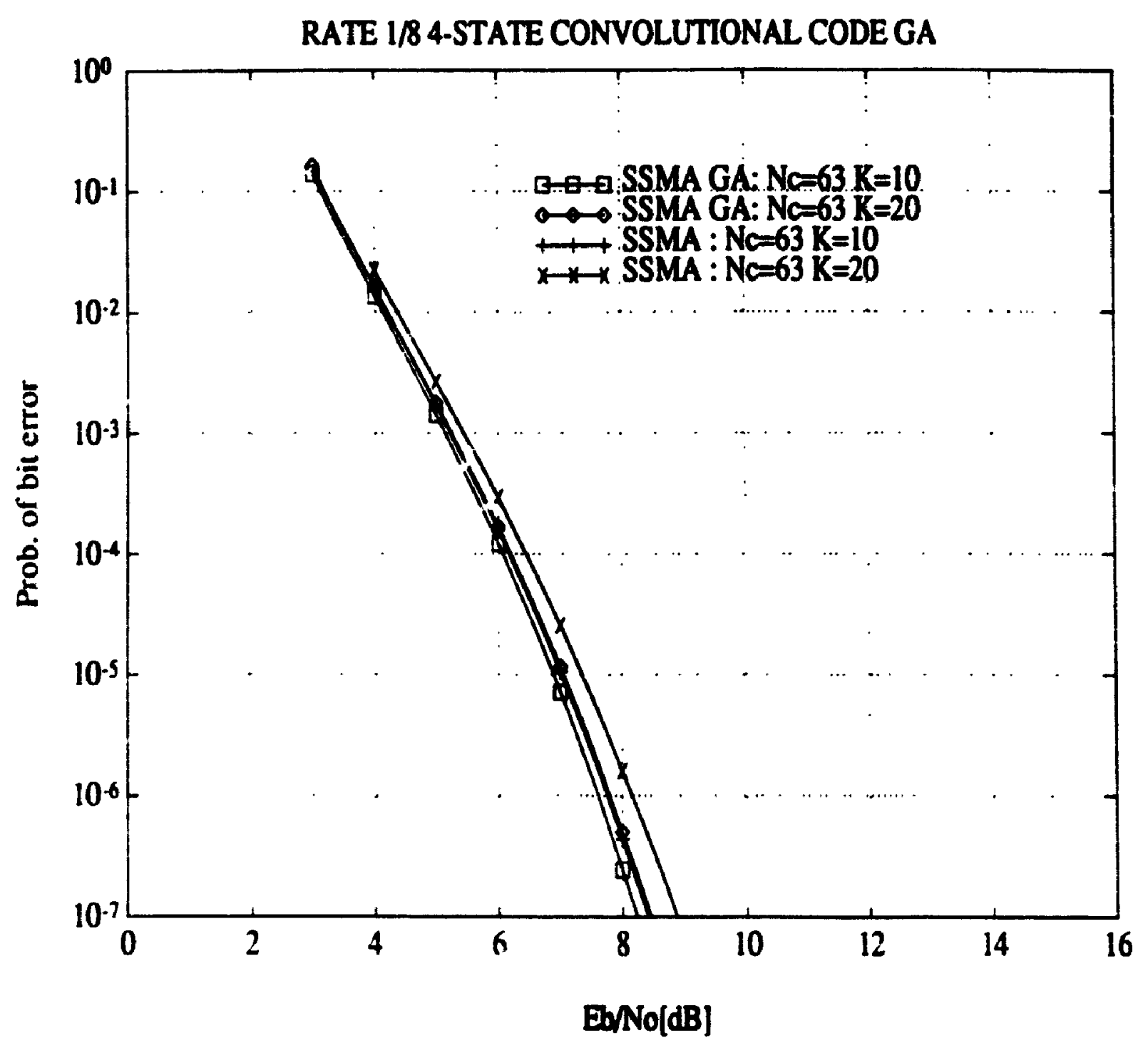

Figure 5.31: Performance of rate 1/8 4-state convolutional code with $K=10$ and $k=20$ users employing the Gaussian Approximation $\left(N_{c}=63\right)$. 


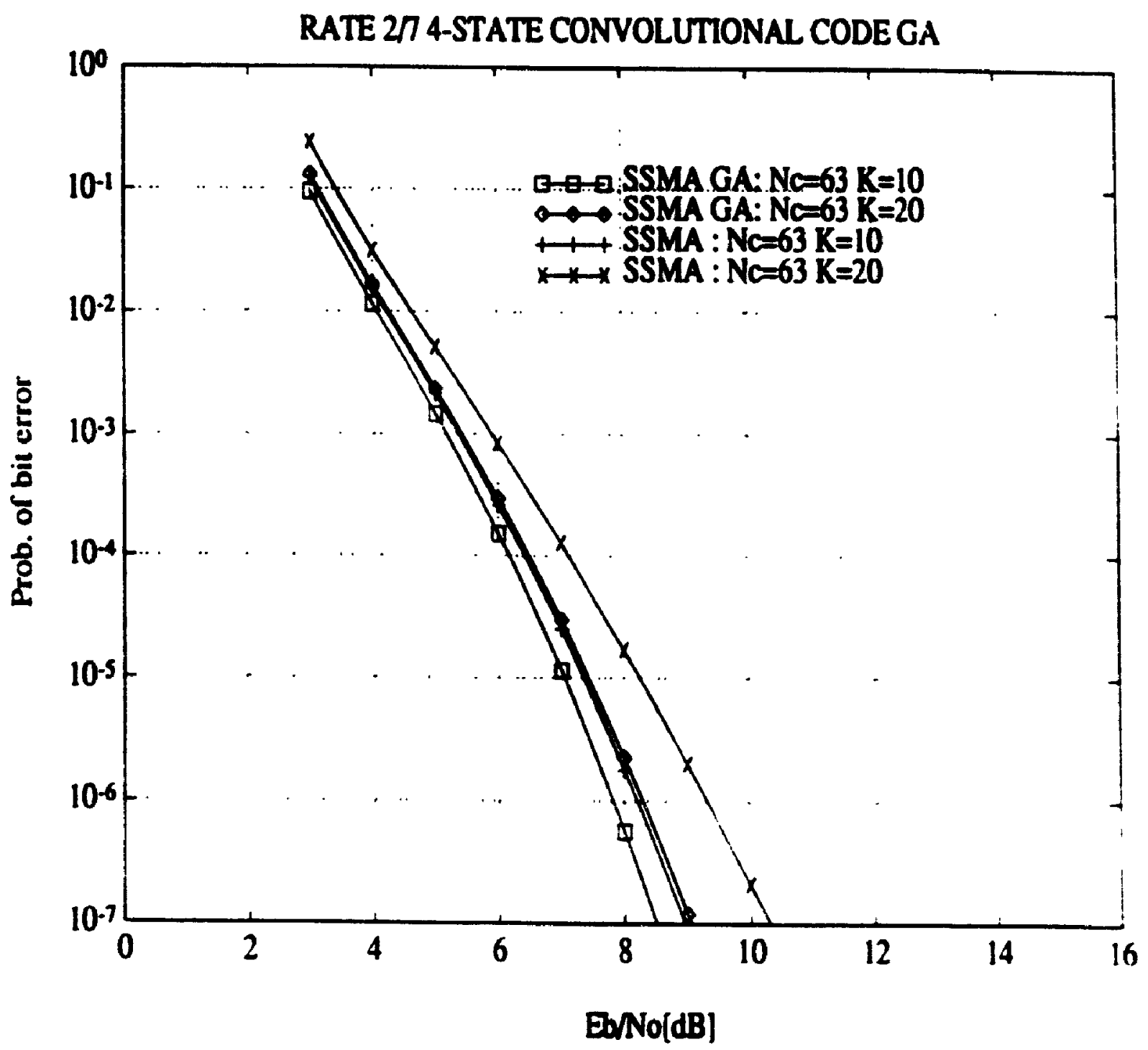

Figure 5.32: Performance of rate 2/7 4-state convolutional code with $K=10$ and $\mathrm{K}=20$ users employing the Gaussian Approximation $\left(N_{c}=63\right)$. 
span a complete PN sequence period. As was explained in Section 2.2. one must average the partial correlation products over all possible subsequences. Thus the evaluation of the performance of such a system is very computationally intensive. even when the subsequence and full sequence length are carefully choosen so as to minimize the number of subsequences that one must average equations (2.26) and (2.27) over. The result presented below is for a SSMA system employing a rate $1 / T$ code in the presence of $K=10$ users. The spreading sequences are Gold codes of length $N_{c}=511$ chips. Each convolutionally coded symbn! is assumed to span one-seventh of the complete PN sequence or a subsequence of length i3 rhips. For this choice of sequence and subsequence lengths there are 49 possible subsequences that must be averaged over (i.e., 73 divides evenly into 511 seven times). The resulting performance curve in Figure $\mathbf{5 . 3 3}$ shows that for this choice of Gold code and subsequence length, that the coded SSMA system with $::=10$ users provides performance within $0.2 \mathrm{~dB}$ of the coded performance with no SSMA at a BER of $10^{-5}$. Thus from this result it would appear that the partial cross correlation products have no more of a deleterious effect than do the full length cross-correlation products. A generalization of this conclusion to all convolutional codes would require further investigation.

\subsection{Summary}

Table 5.3 provides a summary of the performance of the trellis and convolutionally coded SSMA systems at a BER of $10^{-5}$ for $h=20$ users. The performance is compared to the corresponding performance of the uncoded signal modulation that will provide the equivalent throughput (i.e. BPSK for rate $1 / \mathrm{n}$ codes and QPSK for rate $2 / \mathrm{n}$ codes $)$. From the table it can be seen that the low rate convolutional codes provide the best performance of the codes considered. This is in spite of the fact that the low rate convolutional codes must employ shorter PN spreading sequences which leads to a larger IVI contribution. It is interesting to compare the performance in 


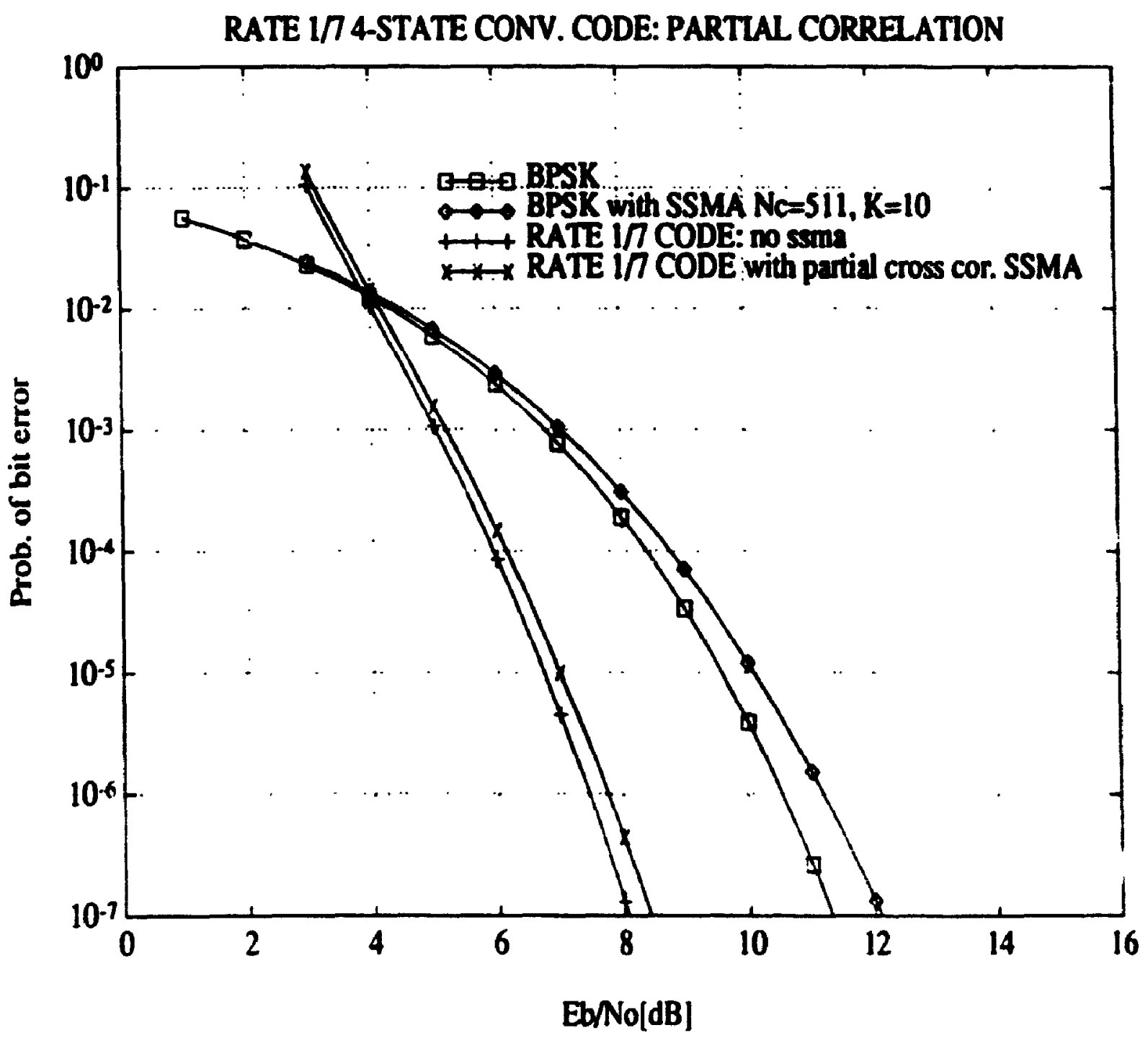

Figure 5.33: Performance of rate 1/7 4-state convolutional corle for $K=10$ users $\left(N_{c}=511\right)$. Each coded symbol spans a subsequence of 73 chips 
Table 5.3 to the minimum distances of the codes as tabulated in Table 3.8, from which it can be seen that the effective minimum distance is an accurate predictor of the performance of the trellis or convolutional codes (i.e. the greater the minimum distance the better the BER for a given SNR). The results in this chapter indicate that the larger distance properties of the low rate convolutional codes more than compensate for the slight degradation due to increased IUI. Specifically the 16-state rate $1 / 8$ and the 16 -state rate $2 / 7$ codes can be seen to provide the best performance for their given rates. On the basis of throughput, the 16 -state rate $2 / 7$ code provides the best performance for a given $\frac{E_{0}}{N_{0}}$. 


\begin{tabular}{|c|c|c|c|c|c|c|}
\hline code & rate & $\begin{array}{c}\text { no. } \\
\text { states }\end{array}$ & $\begin{array}{l}\text { sequence } \\
\text { length }\end{array}$ & $\begin{array}{c}E_{b} / N_{0} \\
\text { for } \\
P_{e}=10^{-s} \\
{[d B]}\end{array}$ & $\begin{array}{c}\text { gain } \\
\text { over } \\
\text { BPSK } \\
\text { [dB] }\end{array}$ & $\begin{array}{c}\text { gain } \\
\text { over } \\
\text { QPSK } \\
\text { [dB] }\end{array}$ \\
\hline $\begin{array}{l}\text { 4-PSK trellis } \\
\text { 4-PSK trellis } \\
\text { 4-PSK trellis }\end{array}$ & $\begin{array}{l}1 / 2 \\
1 / 2 \\
1 / 2\end{array}$ & $\begin{array}{l}2 \\
4 \\
8\end{array}$ & $\begin{array}{l}511 \\
511 \\
511\end{array}$ & $\begin{array}{l}9.9 \\
7.6 \\
6.8\end{array}$ & $\begin{array}{l}0.9 \\
3.2 \\
1.0\end{array}$ & $\cdot$ \\
\hline $\begin{array}{l}\text { convolutional } \\
\text { convolutional } \\
\text { convolutional }\end{array}$ & $\begin{array}{l}1 / 2 \\
1 / 2 \\
1 / 2\end{array}$ & $\begin{array}{c}4 \\
8 \\
16\end{array}$ & $\begin{array}{l}255 \\
255 \\
255\end{array}$ & $\begin{array}{l}7.4 \\
6.7 \\
6.2\end{array}$ & $\begin{array}{l}3.4 \\
4.1 \\
4.6\end{array}$ & - \\
\hline $\begin{array}{l}\text { convol- ional } \\
\text { convolutional } \\
\text { convolutiona }\end{array}$ & $\begin{array}{l}1 / 8 \\
1 / 8 \\
1 / 8\end{array}$ & $\begin{array}{c}4 \\
8 \\
16\end{array}$ & $\begin{array}{l}63 \\
63 \\
63\end{array}$ & $\begin{array}{l}7.4 \\
6.6 \\
5.8\end{array}$ & $\begin{array}{l}3.4 \\
4.2 \\
5.0\end{array}$ & - \\
\hline $\begin{array}{l}\text { 8-PSK trellis } \\
\text { 8-PSK trellis } \\
\text { 8-PSK treilis }\end{array}$ & $\begin{array}{l}2 / 3 \\
2 / 3 \\
2 / 3 \\
\end{array}$ & $\begin{array}{l}4 \\
\times \\
16\end{array}$ & $\begin{array}{l}511 \\
511 \\
511\end{array}$ & $\begin{array}{l}9.6 \\
9.2 \\
8.8\end{array}$ & - & $\begin{array}{l}2.8 \\
3.2 \\
3.6\end{array}$ \\
\hline $\begin{array}{l}\text { convolutional } \\
\text { convolutional }\end{array}$ & $\begin{array}{l}2 / 3 \\
2 / 3 \\
\end{array}$ & $\begin{array}{r}4 \\
16 \\
\end{array}$ & $\begin{array}{l}127 \\
127 \\
\end{array}$ & $\begin{array}{l}9.5 \\
7.8\end{array}$ & - & $\begin{array}{l}2.5 \\
4.6\end{array}$ \\
\hline $\begin{array}{l}\text { convolutional } \\
\text { convolutional }\end{array}$ & $\begin{array}{l}2 / 7 \\
2 / 7\end{array}$ & $\begin{array}{c}4 \\
16\end{array}$ & $\begin{array}{l}63 \\
63\end{array}$ & $\begin{array}{l}8.3 \\
6.5\end{array}$ & - & $\begin{array}{l}4.1 \\
5.9\end{array}$ \\
\hline
\end{tabular}

Table 5.3: Comparison of trellis and convolutionally roded SSMA at $l_{c}=10^{-5}$ for $h=20$ users 


\section{Chapter 6}

\section{Performance Analysis in a Fading Channel}

As was the case for the analysis of the AWGN channel, the key step in the analysis for a fading channel is to formulate and evaluate an expression for the pairwise crror between a transmitted sequence and a possible error sequence (i.e. an error event). Possessing such an expression, the performance of any of the codes under consideration can be evaluated using the appropriate expression for the generalized transfer function bound of equation (3.10). The analysis of this chapter considers a generalized Rician fading channel in which the normalized fading amplitude is defined as $\rho$ (see Appendix $B$ for details of the exact expression for the probability density function of $\rho$ ). Four cases are considered: (i) IUI with channel state information (CSI): (ii) II! without CSI: (iii) use of the Gaussian approximation for the IUI in a fading channel with CSI: and (iv) use of the Gaussian approximation for the IUI in a fading channel with no (SSI. For the channel under consideration. CSI is the value of $\rho$, the normalized fading amplitude. This channel information can be obtained for cxample from the power in a recovered pilot tone.

In a Rician fading channel outputs of the correlation process of the receiver in 
Figure 2.8 (i.e. an analogous expression to equation (2.23)), take on the form

$$
Y_{p}=\rho_{p} X_{p}+Z_{p}+\eta_{p}
$$

in which $\rho_{p}$ is the normalized faded amplitude of the transmitted symbol $\boldsymbol{X}_{\boldsymbol{p}}$. In equation (6.1), the total interuser interference random variable $Z_{p}$ consists of the interuser interference contribution from each of the other users in the SSMA, as in the previous analysis. For the casc of a fading channel it is assumed that the fading on each of the contributions of the interuser interference is independent (i.e. the contributions of IUI arrive on independently faded paths). This slightly alters the definition of $Z_{p}$, since the fading amplitude must now be accounted for. Ising the definition of $G_{p}$ in equation (2.22), $Z_{p}$ can now be expressed as

$$
Z_{p}=\sum_{k=2}^{K} Z_{p}^{k}=\sqrt{E} \cdot \sum_{k=2}^{K} \varphi_{p}^{k} G_{p}^{k}
$$

in which $\varphi_{p}^{k}$ is the normalized fading amplitude on the interuser interference received from the $k^{\text {th }}$ user. It has been assumed without loss of generality that the received signal of the first user is under consideration. Furthermore the notatiun $\varphi_{p}^{k}$ has been employed for the fading amplitudes of the IUl signals to contrast with $\rho_{p}$ the fading amplitude on the transmitted signal. The effert of $\varphi^{k}$ on the performance of the system can be incorporated into the calculation of the moments of $Z_{p}^{k}$. In fact for each moment, all that is required is to calculate the moment of $\varphi^{k}$ and multiply it by the corresponding moment of $Z_{p}$ (this is due to the independence of the moments of $\varphi^{k}$ and $G_{p}^{k}$ ). The details of the calculation of the moments of $\varphi^{k}$ are provided in $\therefore$ ppendix B.

\subsection{Interuser Interference with CSI}

For a Rician fading channel with CSI, it one conditions on the CSI and the int rruser interference $Z_{p}$, the required metric for the evaluation of thro (Chernoff hound can be. 
expressed as

$$
m\left(Y_{p}, X_{p} ; Z_{p}, \rho_{p}\right)=-\left|Y_{p}-\rho_{p} X_{p}\right|^{2}
$$

Incorporating the metric of equation (6.3) into the Chernoff bound of equation (4.12) results in

$$
P(\mathbf{X} \rightarrow \tilde{\mathbf{X}} \mid \mathbf{Z}, \rho) \leq \prod_{p \in \nu} \mathbf{E}\left\{\exp \left[\lambda\left(m\left(Y_{p}, \tilde{X}_{p} ; Z_{p}, \rho_{p}\right)-m\left(Y_{p}, X_{p} ; Z_{p}, \rho_{p}\right)\right)\right]\right\}
$$

in which as before $\nu$ is the set of all $p$ such that $X_{p} \neq \tilde{X}_{p}$. Substituting the definition of the metric from equation (6.3) into equation (6.4) gives

$$
\begin{aligned}
\Gamma(\mathbf{X} \rightarrow \tilde{\mathbf{X}} \mid \mathbf{Z} . \rho) & \leq \prod_{p \in \nu} \mathbf{E}\left\{\exp \left[\lambda\left(-\left|Y_{p}-\rho_{p} \tilde{X}_{p}\right|^{2}+\left|Y_{p}-\rho_{p} X_{p}\right|^{2}\right)\right]\right\} \\
& =\prod_{F \in \nu} \mathrm{E}\left\{\exp \left[-\lambda\left(\rho_{p}^{2}\left(\left|\tilde{X}_{p}\right|^{2}-\left|X_{p}\right|^{2}\right)+2 \rho_{p} R e\left[Y_{p}\left(X_{p}-\tilde{X}_{p}\right)^{*}\right]\right)\right]\right\}
\end{aligned}
$$

Using the definition of $Y_{p}$ of equation (6.1), and substituting it into equation (6.5) gives

$$
\begin{aligned}
P(\mathbf{X} \rightarrow \tilde{\mathbf{X}} \mid \mathbf{Z}, \rho) \leq & \prod_{p \in \nu} \mathbf{E}\left\{\operatorname { e x p } \left[-\lambda\left(\rho_{p}^{2}\left(\left|\tilde{X}_{p}\right|^{2}-\left|X_{p}\right|^{2}\right)+\right.\right.\right. \\
& \left.\left.\left.2 \rho_{p} \operatorname{Re}\left[\left(\rho_{p} X_{p}+Z_{p}+\eta_{p}\right)\left(X_{p}-\tilde{X}_{p}\right)^{*}\right]\right)\right]\right\}
\end{aligned}
$$

which after some algebra takes on the iorm

$$
\begin{aligned}
P(\mathbf{X} \rightarrow \overline{\mathbf{X}} \mid \mathbf{Z} . \rho) \leq & \prod_{p \in \nu} \mathbf{E}\left\{\operatorname { e x p } \left[-\lambda\left(\rho_{p}^{2}\left(\mid X_{p}-\dot{X}_{r i} i^{\hat{2}}\right)+\right.\right.\right. \\
& \left.\left.\left.2 \rho_{p} R c\left[\eta_{p}\left(X_{p}-\tilde{X}_{p}\right)^{*}\right]+2 \rho_{p} \operatorname{Re}\left[Z_{p}\left(X_{p}-\tilde{X}_{p}\right)^{*}\right]\right)\right]\right\}
\end{aligned}
$$

Noting that the random variables $X_{p}, Z_{p}$, and $\eta_{p}$ are all independent, the expectation 
can be split into a product of expectations

$$
\begin{aligned}
P(\mathbf{X} \rightarrow \tilde{X} \mid Z, \rho) \leq & \prod_{p \in \nu} \mathbf{E}\left\{\exp \left[-\lambda\left(\rho_{p}^{2}\left(\left|X_{p}-\tilde{X}_{p}\right|^{2}\right)\right)\right]\right\} \\
& \mathbf{E}\left\{\exp \left[-\lambda\left(2 \rho_{p} \operatorname{Re}\left[\eta_{p}\left(X_{p}-\tilde{X}_{p}\right)^{*} \mid\right)\right]\right\}\right. \\
& \mathbf{E}\left\{\exp \left[-\lambda\left(2 \rho_{p} \operatorname{Re}\left[Z_{p}\left(X_{p}-\tilde{X}_{p}\right)^{*} \mid\right)\right]\right\}\right.
\end{aligned}
$$

The second expectation involving the AWGN has been evaluated in Chapter 4. Using this result (see equation (4.27)), and factoring out the symbol energy term allows one to rewrite equation (6.8) as

$$
\begin{aligned}
P(\mathbf{x} \rightarrow \tilde{\mathbf{x}} \mid \mathbf{z}, \rho) \leq & \prod_{p \in \nu} \exp \left[-E_{q} \therefore \rho_{p}^{2}\left|x_{p}-\bar{x}_{p}\right|^{2}\left(1-\lambda \sigma^{2}\right)\right] \\
& \mathbf{E}\left\{\exp \left[-2 E_{g} \lambda \rho_{p} \operatorname{Re}\left[z_{p}\left(x_{p}-\tilde{x}_{p}\right)^{+}\right]\right]\right\}
\end{aligned}
$$

in which $\sigma^{2}=N_{0}$ is the variance of the complex AWGN. Following the derivation of the Chernoff bound for the AWGN channel, one can evaluate the last expectation of equation (6.9) by decomposing $z_{p}$ into its real and imaginary parts

$$
\left.\mathrm{E}\left\{\exp \left(-2 \lambda \rho_{p} E_{\mathrm{s}} \operatorname{Re}\left\{z_{p}\left(x_{p}-\tilde{x}_{p}\right)^{*}\right\}\right)\right\}=\int_{-\infty}^{\infty} \int_{-\infty}^{\infty} \exp \left(-2 \lambda \rho_{p} E_{\mathrm{s}} \mid z_{l} d_{l}+z_{Q} d_{Q}\right]\right) .
$$

In equation (6.10) (analogous to the case with just AWGN) the in-phase and quadrature components of $z_{p}$ (i.e. $z_{I}$ and $z_{Q}$ ) are in general dependent and as such the moments must be evaluated jointly (i.e. the density $p\left(z_{l}, z_{Q}\right)$ cannot be split into individual density functions of $z_{l}$ and $z_{Q}$ ). Again, defining the randorn variable: $v=z_{I} d_{I}+z_{Q} d_{Q}$, equation (6.10) can be evaluated using the nethod of moments

$$
\begin{aligned}
\mathbf{E}\left\{\exp \left(-2 \lambda \rho_{p} E, \operatorname{Re}\left\{z_{p}\left(x_{p}-\tilde{x}_{p}\right)^{*}\right\}\right)\right\} & =\int_{-\infty}^{\infty} \exp \left(-2 \lambda \rho_{p} E, v\right) p(v) d v \\
& =\sum_{i=1}^{N_{m}} W_{p_{1}} \exp \left(-2 \lambda \rho_{p} E_{g} \zeta_{p_{v}}\right)
\end{aligned}
$$


in which $\zeta_{p_{1}}$ is the $i^{\text {th }}$ node corresponding to the $i^{\text {th }}$ weight $W_{p_{1}}$. The number of moments used in the weighted summation is $N_{m}$. Substituting the result of equation (6.11) into equation (6.9) gives a pairwise error of

$$
\left.P(\mathrm{x} \rightarrow \overline{\mathrm{x}} \mid \rho) \leq \prod_{p \in \nu} \exp \left[-\lambda \rho_{p}^{2} E_{s}\left(1-\lambda N_{0}\right)\left|x_{p}-\tilde{x}_{p}\right|^{2}\right)\right] \cdot \sum_{i=1}^{N_{m}} W_{p_{t}} \exp \left(-2 \lambda \rho_{p} E_{o} \zeta_{p_{v}}\right)
$$

Again, in order to obtain the tightest possible upper bound on the probability of error, equation (6.12) must be optimized with respect to the Chernofi parameter $\lambda$. which is more easily achieved if $\lambda$ is redefined as $\lambda^{\prime} / N_{0}$. Dropping the primes, gives

$$
\left.P(\mathbf{x}-\tilde{\mathbf{x}} \mid \rho) \leq \prod_{p \in \nu} \exp \left[-\rho_{p}^{2} \frac{E_{s}}{V_{0}} \lambda(1-\lambda)\left|x_{p}-\tilde{s}_{p}\right|^{2}\right)\right] \cdot \sum_{i=1}^{N_{m}} W_{p_{0}} \exp \left(-2 \rho_{p} \lambda \frac{E}{V_{0}} ; p_{v}\right)
$$

The bound of equation (6.13) must still be averaged over the amplitude normalizeri fading random variable $\rho_{p}$. If it is assumed that the data symbols are interleaved to a depth that is much greater than length of the fades. it follows that the random variables $\rho_{p}$ are independent from one symbol period to the next. The calculation of the generalized transfer function bound in the presence of fading requires the evaluation of the branch gains of the state transition diagram. averaged over the SSMA interference and the Rician fading. Consider an arbitrary branch in the sequence being bounded in equation (6.13). Dropping the subscript $p$ without any loss of generality and averaging over the normalized Rician density of [119]

$$
p_{\rho}(\rho)=2 \rho\left(1+K_{f}\right) \exp \left[-K_{f}-\rho^{2}\left(1+K_{f}\right)\right] I_{0}\left(2 \rho \sqrt{K_{f}\left(K_{f}+1\right)}\right)
$$

gives a Chernoff bound of

$$
\begin{aligned}
P(x \rightarrow \tilde{x}) \leq & \int_{0}^{\infty} \operatorname{2\rho }\left(1+K_{f}\right) \exp \left[-K_{f}-\rho^{2}\left(1+K_{f}\right)\right] I_{0}\left(2 \rho \sqrt{K_{f}\left(K_{f}+1\right)} .\right. \\
& \left.\exp \left[-\rho^{2} \frac{E_{s}}{V_{0}} \lambda(1-\lambda)|x-\bar{x}|^{2}\right)\right] \cdot \sum_{i=1}^{N_{m}} W_{i} \exp \left(-2 \rho \lambda \frac{E_{g}}{N_{0}} \zeta_{i}\right) d \rho
\end{aligned}
$$

l'sing the integral identity

$$
I_{0}(x)=\frac{1}{\pi} \int_{0}^{\pi} \exp (x \cos (\theta)) d \theta
$$


equation (6.15) can be expressed as

$$
\begin{aligned}
P(x \rightarrow \tilde{x}) \leq & 2\left(1+K_{f}\right) \exp \left(-K_{f}\right) \sum_{i=1}^{N_{m}} W_{i} \cdot \\
& \int_{0}^{\infty} \rho \exp \left\{-\rho^{2}\left[\left(1+K_{f}\right)+\frac{E_{0}}{N_{0}} \lambda(1-\lambda)|x-\dot{x}|^{2}\right]-2 \rho \lambda \frac{E_{0}}{N_{0}} \zeta_{f}\right\} \\
& \frac{1}{\pi} \int_{0}^{\pi} \exp \left[2 \rho \sqrt{K_{f}\left(K_{f}+1\right)} \cos (\theta)\right] d \theta d \rho
\end{aligned}
$$

Defining

$$
\begin{aligned}
& a=1+K_{f}+\frac{E_{a}}{N_{0}} \lambda(1-\lambda)|x-\dot{x}|^{2} \\
& b_{1}=2 \lambda \frac{E_{s}}{N_{0}} \zeta_{i}-2 \sqrt{K_{f}\left(K_{f}+1\right)} \cos (\theta)
\end{aligned}
$$

equation (6.17) can be expressed as

$$
P(x \rightarrow \tilde{x})=2\left(1+K_{f}\right) \exp \left(-K_{f}\right) \sum_{i=1}^{N_{m}} W_{i} \frac{1}{\pi} \int_{0}^{\pi} \int_{0}^{\infty} \rho \exp \left(-a \rho^{2}-b_{i} \rho\right) d \rho d \theta
$$

The double integral expression of equation (6.20) has no closed form solution, however it can be expressed in the form of the following finite integral (sec Appendix $\left(^{\circ}\right.$ )

$$
P(x \rightarrow \tilde{x})=\left(1+K_{f}\right) \exp \left(-K_{f}\right) \sum_{i=1}^{N_{m}} W_{i}\left[\frac{1}{a}-\frac{1}{\sqrt{\pi}} \int_{0}^{\pi} \frac{c_{i}}{\sqrt{a}} \exp \left(\alpha \cdot c_{i}^{2}\right) \operatorname{erfc}\left(\sqrt{a} \cdot c_{i}\right) d \theta\right]
$$

in which

$$
c_{\mathrm{s}}=\frac{b_{\mathrm{z}}}{2 a}
$$

The Chernoff bound of equation (6.21) can be optimized over $\lambda$ using numerical techniques, for a given value of Euclidean distance $|x-\dot{x}|$. However, siner $\lambda$ is a function of Euclidean distance. the optimization of equation (6.13) with respeet to $\lambda$ is a function of the sequence length $\boldsymbol{N}^{\prime}$. In order to make the evaluation of in transfer function bound tractable. $\lambda$ has been optimized for the minimum branch distauce. and this value of $\lambda$ was employed for all branclues of the transfer function bound. 
This results in a suboptimal Chernoff bound, however the resulting bound is still sufficiently tight to provide useful results.

\subsection{Interuser Interference without CSI}

In a fading channel in which no CSI is available. the outputs of the correlation process in the receiver take on the form (as in the case in which CSI is available)

$$
Y_{p}=\rho_{p} X_{p}+Z_{p}+\eta_{p}
$$

The interuser interference random variable $Z_{p}$ has the same form as was discussed in Section 6.1. When the receiver has no CSI in a Rician fading channel, the metric at the output of the $I$ and $Q$ correiators takes on the form

$$
m\left(Y_{p}, X_{p}\right)=-\left|Y_{p}-X_{p}\right|^{2}
$$

Incorporating the metric of equation (6.24) into the Chernoff bound of equation (4.12) results in (analogous to equation (6.4))

$$
P(\mathbf{X} \rightarrow \overline{\mathbf{X}} \mid \mathbf{Z}, \rho) \leq \prod_{p \in \nu} \mathbf{E}\left\{\exp \left[\lambda\left(m\left(Y_{p}, \tilde{X}_{p}\right)-m\left(Y_{p}, X_{p}\right)\right)\right]\right\}
$$

in which $\nu$ is the set of all $p$ such that $X_{p} \neq \bar{X}_{p}$. Substituting the definition of the metric from equation (6.24) into equation (6.25) gives

$$
\begin{aligned}
P(X \rightarrow \tilde{X} \mid Z . \rho) & \leq \prod_{p \in \nu} E\left\{\exp \left[\lambda\left(-\left|Y_{p}-\tilde{X}_{p}\right|^{2}+\left|Y_{p}-X_{p}\right|^{2}\right)\right]\right\} \\
& =\prod_{p \in \nu} E\left\{\exp \left[-\lambda\left(\left|\dot{X}_{p}\right|^{2}-\left|X_{p}\right|^{2}+2 \operatorname{Re}\left[Y_{p}\left(X_{p}-\tilde{X}_{p}\right)^{*}\right]\right)\right]\right\}
\end{aligned}
$$

l'sing the definition of $Y_{p}$ of equation (6.23), and substituting it into equation (6.26) gives:

$$
P(\mathbf{X} \rightarrow \overline{\mathbf{X}} \mid \mathbf{Z} . \rho) \leq \prod_{p \in \nu} \mathbf{E}\left\{\operatorname { e x p } \left[-\lambda\left(\left|\dot{\mathbf{X}}_{p}\right|^{2}-\left|X_{p}\right|^{2}+\right.\right.\right.
$$




$$
\left.\left.\left.2 R e\left[\left(\rho_{p} X_{p}+Z_{p}+\eta_{p}\right)\left(X_{p}-\dot{X}_{p}\right)^{\bullet}\right]\right)\right]\right\}
$$

which after some algebra takes on the form

$$
\begin{aligned}
P(\mathbf{X} \rightarrow \overline{\mathbf{X}} \mid Z, \rho) \leq & \prod_{p \in \nu} \mathbf{E}\left\{\operatorname { e x p } \left[-\lambda\left(2(\rho-1)\left|X_{p}\right|^{2}+\left|\tilde{X}_{p}\right|^{2}-2 \rho \operatorname{Re}\left(X_{p} \dot{X}_{p}\right)^{*}+\right.\right.\right. \\
& \left.\left.\left.2 \operatorname{Re}\left[\eta_{p}\left(X_{p}-\tilde{X}_{p}\right)^{*}\right]+2 \operatorname{Re}\left[Z_{p}\left(X_{p}-\tilde{X}_{p}\right)^{*}\right]\right)\right]\right\} \\
\leq & \prod_{p \in \nu} \mathbf{E}\left\{\exp \left[-\lambda\left(\left|X_{p}-\tilde{X}_{p}\right|^{2}+2(\rho-1) \operatorname{Re}\left[X_{p}\left(X_{p}-\dot{X}_{p}\right)^{*}\right)\right]\right\}\right. \\
& \mathbf{E}\left\{\exp \left[-2 \lambda \operatorname{Re}\left[\eta_{p}\left(X_{p}-\tilde{X}_{p}\right)^{*}\right]\right]\right\} \\
& \mathbf{E}\left\{\exp \left[-2 \lambda \operatorname{Re}\left[Z_{p}\left(X_{p}-\tilde{X}_{p}\right)^{*}\right]\right]\right\}
\end{aligned}
$$

In equation (6.28) the expectation has been split into a product of three exp. tations since the random variables $X_{p}, Z_{p}$, and $\eta_{p}$ are all independent. The argul . . . of the first expectation in equation $(6.28)$ can be simplified by noting that $\left|X_{p}\right|^{2}=\left|\tilde{X}_{p}\right|^{2}$ for the constant envelope MPSK signal constellations that are to be ronsidered in this study. Under these conditions it follows that

$$
\left|X_{p}-\tilde{X}_{p}\right|^{2}=2 R e\left\{X_{p}\left(X_{p}-\dot{X}_{p}\right)\right\}
$$

Furthermore. the second and third expectations of expation (6.28) are of the same form as those in equation (6.8), except for the lack of the presence of the random variable $\rho$. Thus using the results of equations (6.9) and (6.11), as wrll as the simplification of equation (6.29), one obtains from equation (6.2א)

$$
P(\mathbf{x} \rightarrow \tilde{\mathbf{x}} \mid \rho) \leq \prod_{p \in \nu} \exp \left[-\lambda E_{s}\left|x_{p}-\tilde{s}_{p}\right|^{2}\left(\rho-\lambda N_{0}\right)\right] \cdot \sum_{i=1}^{N_{m}} \mid W_{p_{0}}\left(\cdot x_{p}\right)\left(-2 \lambda l,_{,} \zeta_{p_{0}}\right)
$$

As in Section 6.1, in order to obtain the tighterst possible upper bonnd on the probability of error, equation (6.30) must be optimizerl with respeect to the (ihernoff 
parameter $\lambda$, which is more easily achieved if $\lambda$ is redefined as $\lambda^{\prime} / N_{0}$. Dropping the primes, gives

$$
P(x \rightarrow \tilde{x} \mid \rho) \leq \prod_{p \in \nu} \exp \left[-\lambda \frac{E_{i}}{N_{0}}\left|x_{p}-\tilde{x}_{p}\right|^{2}(\rho-\lambda)\right] \cdot \sum_{i=1}^{N_{m}} W_{p_{1}} \exp \left(-2 \lambda \frac{E_{\theta}}{N_{0}} \zeta_{p_{0}}\right)
$$

A veraging equation (6.31) over the normalized amplitude fading random variable $\rho_{p}$ under the same conditions defined in Section 6.1 gives

$$
\begin{aligned}
P(x \rightarrow \tilde{x}) \leq & \int_{0}^{\infty} 2 \rho\left(1+K_{f}\right) \exp \left[-K_{f}-\rho^{2}\left(1+K_{f}\right)\right] I_{0}\left(2 \rho \sqrt{K_{f}\left(K_{f}+1\right)}\right) \\
& \exp \left[-\lambda \frac{E_{s}}{N_{0}}|x-\tilde{x}|^{2}(\rho-\lambda)\right] \cdot \sum_{i=1}^{N_{m}} W_{i} \exp \left(-2 \lambda \frac{E_{s}}{N_{0}} \zeta_{i}\right) d \rho
\end{aligned}
$$

In equation (6.32) the subscript $p$ has been dropped without loss of generality. Using the integral identity of equation (6.16), allows equation (6.32) to be rewritten as

$$
\begin{aligned}
P(x \rightarrow \dot{x}) \leq & 2\left(1+K_{f}\right) \exp \left(-K_{f}\right) \sum_{i=1}^{N_{m}} W_{i} \exp \left[-\lambda \frac{E_{s}}{N_{0}}\left(2 \zeta_{p_{i}}-\lambda|x-\tilde{x}|^{2}\right)\right] \cdot \\
& \int_{0}^{\infty} \rho \exp \left\{-\rho^{2}\left(1+K_{f}\right)-\rho \lambda \frac{E_{s}}{N_{0}}|x-\dot{x}|^{2}\right\} . \\
& \frac{1}{\pi} \int_{0}^{\pi} \exp \left[2 \rho \sqrt{K_{f}\left(K_{f}+1\right)} \cos (\theta)\right] d \theta d \rho
\end{aligned}
$$

Delining

$$
\begin{aligned}
& a=1+K_{f} \\
& b=\lambda \frac{E_{s}}{\grave{T}_{0}}|x-\dot{x}|^{2}-2 \sqrt{K_{f}\left(K_{f}+1\right)} \cos (\theta)
\end{aligned}
$$

equation (6.33) can be expressed as

$$
\begin{aligned}
P(x \rightarrow \dot{x})= & 2\left(1+K_{f}\right) \exp \left(-K_{f}\right) \sum_{i=1}^{N_{m}} \mid W_{i} \exp \left[-\lambda \frac{E_{s}}{N_{0}}\left(2 \zeta_{p_{t}}-\lambda\right)|x-\tilde{x}|^{2}\right] . \\
& \frac{1}{\pi} \int_{0}^{\pi} \int_{0}^{\infty} \rho \exp \left(-a \rho^{2}-b \rho\right) d \rho d \theta
\end{aligned}
$$

As was the case in Section 6.1 equation (6.36) cannot be evaluated in a closed form 
solution. however it can be expressed in the form of the following finite integral (see Appendix $C$ ) by using the result of equation (C.T)

$$
\begin{aligned}
P(x \rightarrow \dot{x})= & \left(1+K_{f}\right) \exp \left(-K_{f}\right) \sum_{i=1}^{N_{m}} \mid V_{i} \exp \left[-\lambda \frac{E_{\Delta}}{V_{0}}\left(2 \zeta_{m}-\lambda\right)|x-\dot{x}|^{2}\right] \\
& {\left[\frac{1}{a}-\frac{1}{\sqrt{\pi}} \int_{0}^{\pi} \frac{c}{\sqrt{a}} \exp \left(a \cdot c^{2}\right) \operatorname{erfc}(\sqrt{a} \cdot c) d \theta\right] }
\end{aligned}
$$

in which

$$
c=\frac{b}{2 a}
$$

Proceeding as previously, the Chernoff bound of equation (6.37) can be optimized with respect to the parameter $\lambda$ numerically. Furthermore, for the sake of simplicity, the value of $\lambda$ employed to evaluate equation (6.31) was chosen in an identical manner to that described at the conclusion of Section 6.1.

\subsection{Fading channel with Gaussian assumption and CSI}

The derivation of the (hernoff bound for a Rician fading channel assuming ('SI and also assuming that the interuser interference is Caussian, is analogous to the: analysis of Divsalar and Simon[114]. The only difference involves the ralculation of the variance of the Gaussian random variable which is now a combination of the AWGN and the variance of the interuser interference. The derivation of the ("hernoff bound conditioned on the normalized Rician fading amplitude is identical to the derivation in Section 6.1 up to equation (6.9). Lising a Gaussian approximation for the interuser interference random variable $z_{p}$, equation (6.10) ran be rewritten as

$$
\mathbf{E}\left\{\exp \left(-2 \lambda \rho_{p} E_{s} \operatorname{Re}\left\{z_{o}\left(x_{p}-\tilde{x}_{p}\right)^{-}\right\}\right)\right\} \approx \exp \left(2 \lambda^{2} \rho_{p}^{2} E_{s}^{2} \sigma_{z}^{2}\left|x_{p}-\dot{s}_{p}\right|^{2}\right)
$$

in which $\sigma_{z}^{2}=\frac{1}{2} E\left[\left|z_{p}\right|^{2}\right]$. As in the derivation of the (iaussian approximation in all 
AWGN channel it has been assumed that the in-phase and quadrature components of the interusers interference $\left(z_{l}\right.$ ard $\left.z_{Q}\right)$ are independent. Substituting the result of equation (6.39) into equation (6.9) gives the following approximation for the Chernoff bound

$$
P(\mathrm{x} \rightarrow \overline{\mathrm{x}} \mid \rho) \leq \prod_{p \in \nu} \exp \left[-\lambda \rho_{p}^{2} E_{s}\left(1-2 \lambda\left(N_{0} / 2+\sigma_{z}^{2} E_{\bullet}\right)\right)\left|x_{p}-\tilde{x}_{p}\right|^{2}\right]
$$

Optimizing equation (6.40) with respect to the Chernoff parameter $\lambda$ gives a value of

$$
\lambda_{s p t}=\frac{1}{4\left(N_{0} / 2+\sigma_{z}^{2} E_{0}\right)}
$$

Substituting the value of $\lambda_{\text {spt }}$ into equation $(6.40)$ results in

$$
P(\mathbf{x} \rightarrow \overline{\mathbf{x}} \mid \rho) \leq \prod_{p \in \nu} \exp \left\{-\frac{\rho_{p}^{2}\left|x_{p}-\bar{x}_{p}\right|^{2}}{4\left(\frac{v_{p}}{E_{0}}+\mathrm{E}\left[\left|\tilde{z}_{p}\right|^{2}\right]\right)}\right\}
$$

which can be rewritten in the form

$$
P(x-\bar{x} \mid \rho) \leq \exp \left\{-\frac{1}{1\left(\frac{V_{0}}{E_{0}}+E\left[\left|z_{p}\right|^{2}\right]\right)}\right\}^{\sum_{p \epsilon_{\nu}} \rho_{p}^{2}\left|x_{p}-\dot{x}_{p}\right|^{2}}
$$

As in the previous fading analysis assume that the normalized Rician fading random variables $\rho_{n}$, are independent (i.e. through the use of interleaving). In the calculation of the gencralizeri transfer function bound for a particular code in the presence of fading and SSIIA. the branch label gains in an AllGN channel must be replaced with branch label gains averaged over the SSMA interuser interference and the Rician fading. ("hoosing an arbitrary Euclidean distance for the branch gain and dropping the subscript $p$ without any loss of generality, gives a Chernoff bound for a given branch of

$$
\begin{aligned}
P(x \rightarrow \dot{x}) & \leq \mathbf{E}\left\{\exp \left(-\rho^{2} \Lambda_{f} \gamma\right)\right\} \\
& \leq \int_{0}^{\infty} \exp \left(-\rho^{2} \Lambda_{f} \gamma\right) p(\rho) d \rho
\end{aligned}
$$


in which $p(\rho)$ is the probability density function of the normalized Rician fading amplitude. Furthermore

$$
\begin{aligned}
\Lambda_{f} & =|x-\dot{x}|^{2} \text { and } \\
\gamma & =\frac{1}{4\left(\frac{N_{0}}{E_{a}}+E\left[\left|z_{p}\right|^{2}\right]\right)}
\end{aligned}
$$

The expectation of equation (5.44) has been evaluated by Divsalar and Simon(114). For the sake of completeness, the derivation of this result has been reproduced below.

Substituting the normalized Rician amplitude density function into muation (6.14) results in

$$
\begin{aligned}
P(x \rightarrow \tilde{x}) \leq & \int_{0}^{\infty} \exp \left(-\rho^{2} \Lambda_{f} \gamma\right) \\
& 2 \rho\left(K_{f}+1\right) \cdot \exp \left[-\rho^{2}\left(K_{f}+1\right)-K_{f}\right] . \\
& I_{0}\left(2 \rho \sqrt{K_{f}\left(K_{f}+1\right)}\right) d \rho
\end{aligned}
$$

in which $I_{0}(\cdot)$ is the zeroth order modified Bessel function of the lirst kind (ser Appendix $B$ for further details). Using the fact that $I_{0}(x)$ can be expressod as an infinite series of the form

$$
I_{0}(x)=\sum_{n=0}^{\infty} \frac{1}{(n !)^{2}} \cdot\left(\frac{x}{2}\right)^{2}
$$

allows equation (6.4i) to be expressed in the form

$$
\begin{aligned}
P(x \rightarrow \dot{r}) \leq & 2\left(1+K_{f}\right) \exp \left(-K_{f}\right) \cdot \\
& \int_{0}^{\infty} \sum_{n=0}^{\infty} \frac{\rho^{2 n+1}}{(n !)^{2}} \cdot\left[K_{f}\left(K_{f}+1\right)\right]^{n} \cdot \exp \left[-\rho^{2}\left(1+K_{f}+\Lambda_{f} \gamma\right)\right] d \rho
\end{aligned}
$$

Equation (6.49) can be simplified through use of the following integral identity|117]

$$
\int_{0}^{\infty} x^{2 n+1} \exp \left(-o x^{2}\right) d x=\frac{n !}{2 \phi^{n+1}}
$$

Noting that in equation (6.49) $0=1+k_{f}+\Lambda_{f} \gamma$, the pairwise arror can be simplified 
10

$$
\begin{aligned}
P(x \rightarrow i) & \leq 2\left(1+K_{f}\right) \exp \left(-K_{f}\right) \sum_{n=0}^{\infty} \frac{\left(K_{f}\left(K_{f}+1\right)\right]^{n}}{(n !)^{2}} \cdot \frac{n !}{2\left(1+K_{f}+\Lambda_{f} \gamma\right)^{n+1}} \\
& \leq \frac{1+K_{f}}{1+K_{f}+\Lambda_{f} \gamma} \exp \left(-K_{f}\right) \sum_{n=0}^{\infty} \frac{1}{n !} \cdot\left[\frac{K_{f}\left(K_{f}+1\right)}{1+K_{f}+\Lambda_{f} \gamma}\right]^{n} \\
& \leq \frac{1+K_{f}}{1+K_{f}+\Lambda_{f} \gamma} \exp \left(-K_{f}\right) \exp \left[\frac{K_{f}\left(K_{f}+1\right)}{1+K_{f}+\Lambda_{f} \gamma}\right] \\
& \leq \frac{1+K_{f}}{1+K_{l}+\Lambda_{f} \gamma} \exp \left[\frac{-K_{f} \Lambda_{f} \gamma}{1+K_{f}+\Lambda_{f} \gamma}\right]
\end{aligned}
$$

\subsection{Fading channel with Gaussian assumption and no CSI}

The derivation of the Chernoff bound for a Rician fading channel assuming that no ( $\mathrm{SI}$ is present as well as that the interuser interference is Gaussian. is also analogous 1o that of Divsalar and Simoni114!. As in Section 6.3 the only difference involves the -aiculation of the variance of the (iaussian random variable which is now a combination of the AlHGS and the variance of the interuser interference. The derivation of the (hernoff bound conditioned on the normalized Rician fading amplitude when no ('SI is present is identical to the derivation in Section 6.2 up to equation (6.30). I'sing the (iaussian approximation for the interuser interference random variable $Z_{p}$. is expressed in equation (4.32) allows one to write equation (6.30) in the following approximate form

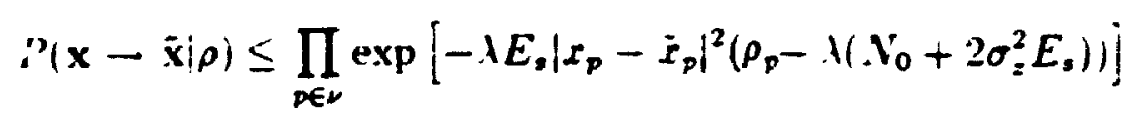

Is in 6.1 and Section 6.2. the parameter $A$ cannot be optimized independently of 
the fading parameter $\rho$. Define

$$
\lambda^{\prime}=\frac{\lambda}{N_{0}+2 \sigma_{\Sigma}^{2} E}
$$

Dropping the primes and substituting equation (6.53) into equation (6.52) gives

$$
\begin{aligned}
P(x \rightarrow \tilde{x} \mid \rho) & \leq \prod_{p \in \nu} \exp \left[-\frac{E_{z}}{N_{0}+2 \sigma_{z}^{2} E_{p}}\left|x_{p}-\dot{x}_{p}\right|^{2} \lambda\left(\rho_{p}-\lambda\right)\right] \\
& \leq \prod_{p \in \nu} \exp \left[-\frac{\left|x_{p}-\dot{x}_{p}\right|^{2}}{\left.\frac{N_{Q}}{\varepsilon_{p}}+\left.\left.E\right|_{p}\right|^{2}\right]} \cdot \lambda\left(\rho_{p}-\lambda\right)\right]
\end{aligned}
$$

As before assume that the normalized Rician fading random variables $\rho_{p}$ are independent from one symbol to the next. Thus the average branch gain for an arbitrary Euclidean distance can be bounded iby (dropping the sulsscript p)

$$
P(x \rightarrow \bar{x}) \leq \exp \left(\lambda^{2} \gamma|x-\bar{x}|^{2}\right) \mathrm{E}\left\{\exp \left(-\rho \lambda \gamma|x-\dot{x}|^{2}\right)\right\}
$$

in which the expectation is over the normalized Rician fading amplitude $p$ and

$$
\gamma=\frac{1}{\left.\left.\frac{N_{0}}{E_{e}}+\mathbf{E} \|\left. z\right|^{2}\right\}\right)}
$$

A veraging over $\rho$ gives

$$
\begin{aligned}
P(x \rightarrow \check{x}) \leq & \left.\exp \left(\lambda^{2} \gamma|x-\tilde{x}|^{2}\right) \int_{0}^{\infty} 2 \rho\left(1+K_{f}\right) \exp \mid-K_{j}-\rho^{2}\left(1+K_{f}\right)\right] \\
& I_{0}\left(2 \rho \sqrt{K_{f}\left(K_{f}+1\right)}\right) \exp \left(-\rho \lambda \gamma|x-\dot{y}|^{2}\right) d \rho
\end{aligned}
$$

Substituting the integral identity of equation (6.16) into rquation (6.50) resislts in

$$
\begin{aligned}
P(x \rightarrow \dot{x}) \leq & 2\left(1+K_{f}\right) \exp \left(\lambda^{2} i|x-\tilde{s}|^{2}-K_{f}\right) . \\
& \int_{0}^{\infty} \rho \exp \left\{-\rho^{2}\left(1+K_{f}\right)-\rho \lambda \gamma|x-\dot{s}|^{2}\right\} . \\
& \frac{1}{\pi} \int_{0}^{\pi} \exp \left[2 \rho \sqrt{K_{f}\left(K_{f}+1\right)} \cos (\theta)\right] d \theta d \rho
\end{aligned}
$$

Following the derivations of Section 6.1 and Sertion 6.2, 
define

$$
\begin{aligned}
& a=1+K_{f} \\
& b=\lambda \gamma|x-\bar{x}|^{2}-2 \sqrt{K_{f}\left(K_{f}+1\right)} \cos (\theta)
\end{aligned}
$$

This allows equation (6.58) to be expressed as

$$
\begin{aligned}
P(x \rightarrow \bar{x})= & 2\left(1+K_{f}\right) \exp \left(\lambda^{2} \gamma|x-\tilde{x}|^{2}-K_{f}\right) \\
& \frac{1}{\pi} \int_{0}^{\pi} \int_{0}^{\infty} \rho \exp \left(-a \rho^{2}-b \rho\right) d \rho d \theta
\end{aligned}
$$

Ising the rosult of cruation (C.T) enables equation (6.61) to be expressed as

$$
\begin{aligned}
P(x \rightarrow \dot{x})= & \left(1+K_{j}\right) \exp \left(\lambda^{2} \gamma|x-\dot{x}|^{2}-K_{f}\right) . \\
& {\left[\frac{1}{a}-\frac{1}{\sqrt{\pi}} \int_{0}^{\pi} \frac{c}{\sqrt{a}} \exp \left(a \cdot c^{2}\right) \operatorname{erfc}(\sqrt{a} \cdot c) d \theta\right] }
\end{aligned}
$$

in which

$$
c=\frac{b}{2 a}
$$

The optimization of cruation $(6.54)$ with respect to $\lambda$ in conjunction with equation (ti.(i2) was carriol out in an identical manner to that described in Section 6.1. 


\section{Chapter 7}

\section{Performance Results in a Rician Fading Channel}

This chapter presents the performance results of the trellis and convolutional codes (described in Chapter 3), in the presence of impairments due to AWGN, IUI from the SSMA and Rician fading. Following the same approach as was used to obtain the results of Chapter 5 , the generalized transfer function Lound algorithm described in Chapter 3 was employed in conjunction witi the Chernoff bounds for pairwise errors analyzed in Chapter 6 . Results for the four cases of Chapter 6 are presenteri below. The performance of the codes is based on the same criteria of expal complexity and throughput as was described in Chapters 2 and 5.

\subsection{Benchmark of Fading Bounds}

Figure 7.1 illustrates the performance of the 2-state 4-PSK corde in a Mician fading, channel both with and without CSI. Curves are plotted for $K$, values of 10.0 and 0.0 (i.e. Rayleigh fading), as well as with no fading. All of these cur oes represant performance bcunds with no ICI from SSMA users. These results are in agrerment. with those of Divsalar and Simon[114]. 


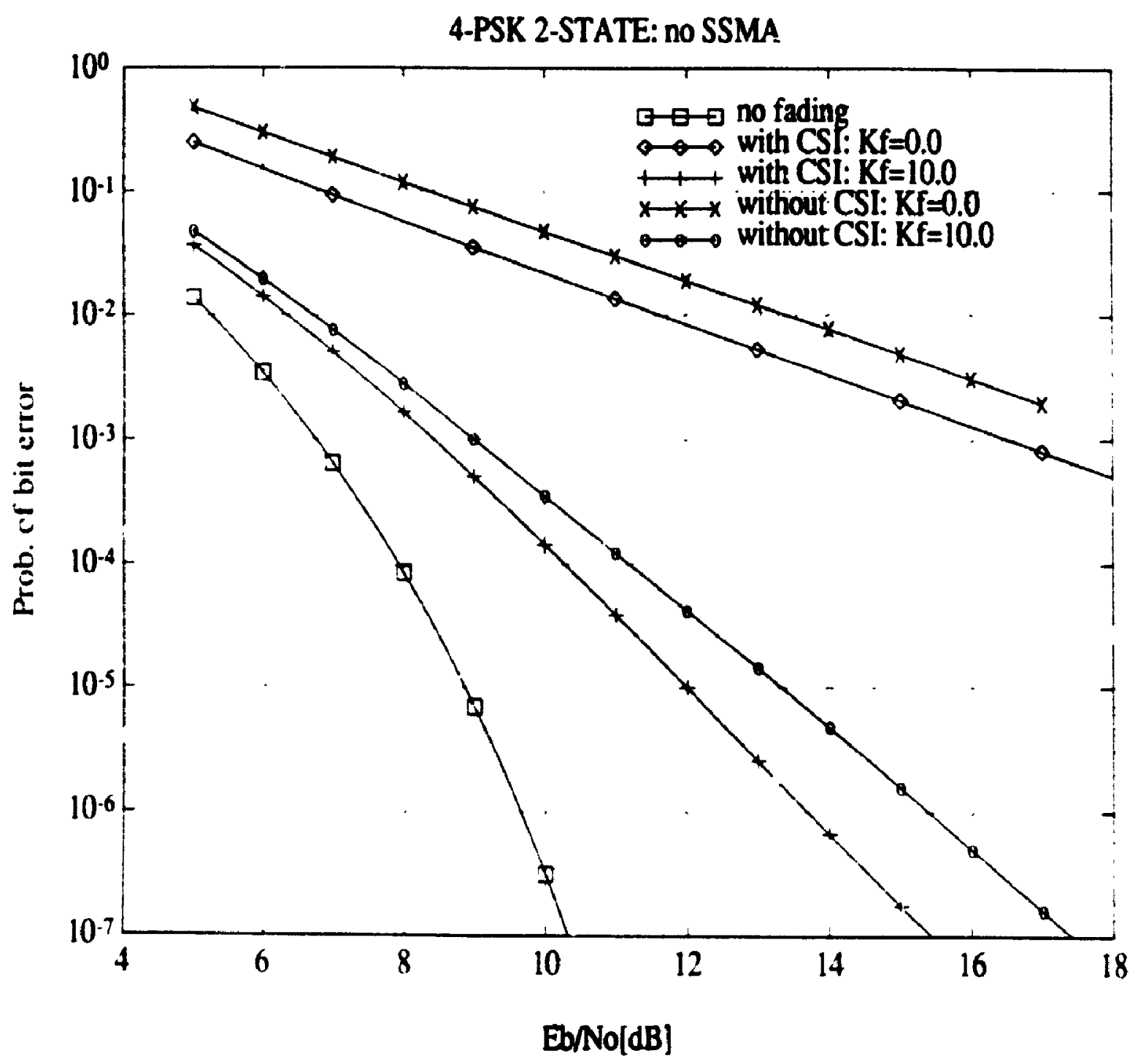

Figure 7.1 : Performance of the 2-state 4-PSK trellis code in Rician fading without SSMA 
Other examples of the performance of coded systems in a Rician fadiug channel without any SSMA are illustrated in Figure 7.2, for the 4-state 4-PSK corte, aud Figure 7.3 for the 4 -state rate $1 / 8$ convolutional code. The important point to note from these figures is that the fading channel alone significantly degrades the performance of the coded systems for the 4-PSK codes, but to a lesser degree for the rate $1 / 8$ corvolutional code. For example under conditions of Rayleigh fading $\left(K_{f}^{\prime}=0.0\right)$, there is about a $2.5 \mathrm{~dB}$ degradation in performance over all $A W G N$ channel for a $P_{e}$ of $10^{-5}$ as opposed to over $14 \mathrm{~dB}$ of degradation for the 4 -state 4-PSK code.

Figures 7.4 to 7.7 illustrate the performance of uncoded BPSK and 4-I'SK operating in a Rician fading channel with and without (SI. It is also assumed that IUI due to $K=20$ SSMA users is present. Gold spreading sequences of length $V_{c}=511$ chips are assumed to be employed by the users. Performance curves are plotted for $K_{f}$ values of $10.0,1.0$, and 0.0 . It ran be observed that the presence of Rician fading and IUI causes significant degradation. Even a moderat.* amount of fading (i.e. $K_{f}=10.0$ ) causes the uncoded BPSK to saturate betwern a $P_{e}$ of $10^{-3}$ and $10^{-4}$. whereas the 4-PSK curve saturates betwen $10^{-2}$ and $10^{-1}$. The presernce of more severe fading (i.e. $K_{f}=1.0$ or 0.0 ) results in saturation at or abovr a $l^{\prime}$. of $10^{-2}$. The important points to note are that for the uncoded case. the presence of CSI provides little improvement is performance, and that the majority of the derradation is due to the fading channel as opposed to the ILI (i.e. compare these results to Figure 5.5 ).

\subsection{Trellis Codes with SSMA in Rician Fading}

Figures 7.8, 7.9. 7.10, 7.11. 7.12, and 7.13 illustrate the perfe rmance of the follo, wing. trellis codes respectively: 1-state 1-PSK with ('SI: 1-state 1-PSK withont 'SI: 1 state 8-PSK with CSI: 1-state X-PSK withont ('SI: I6-state a JSF with ("SI artel 16-state 8-PSK without CSI. Each of the figures has heren ploterd with a sel of 


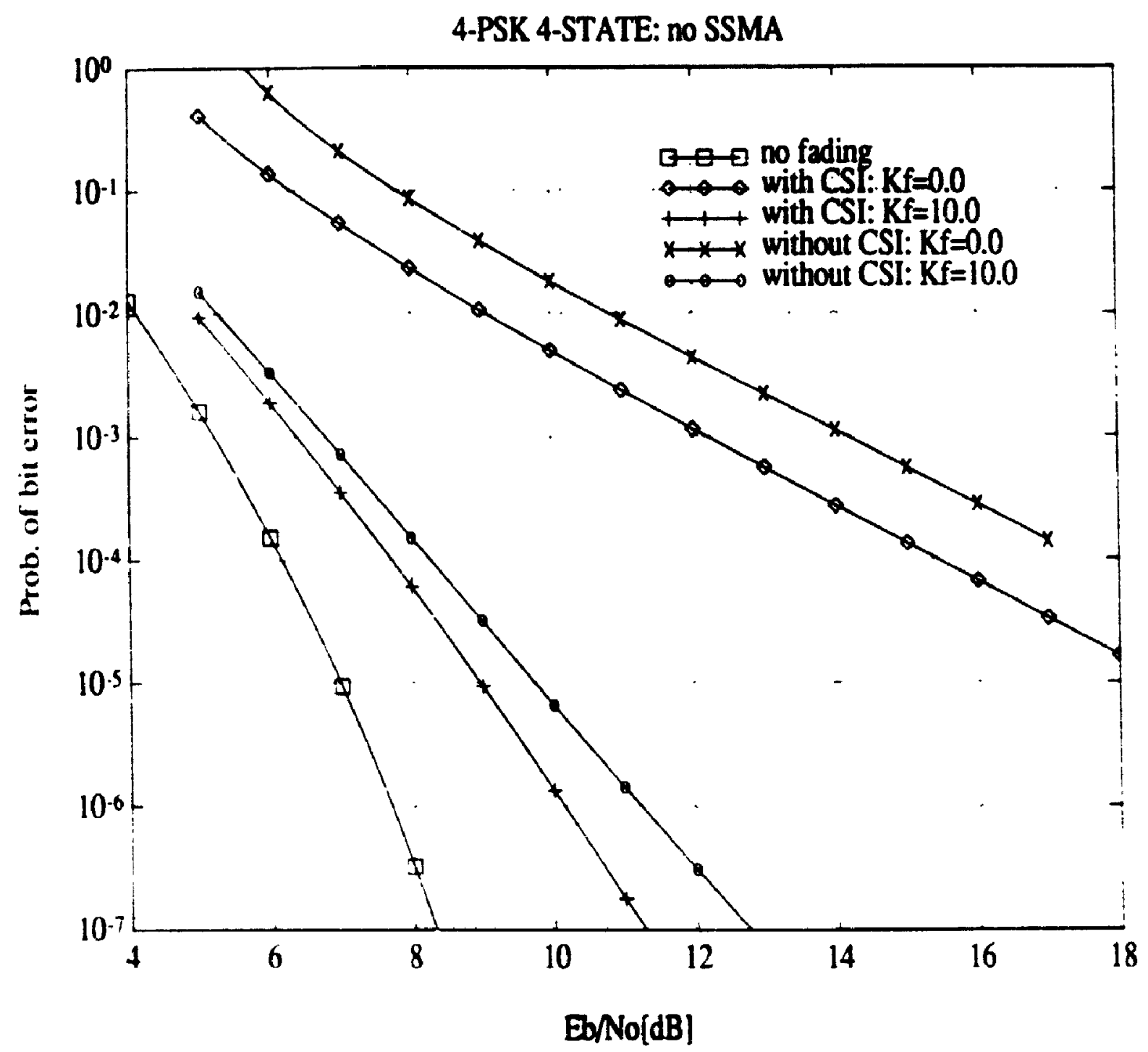

Figure $7: 2:$ Performance of the 4-state 4-PSK trellis code in Rician fading without $\operatorname{SSMA}$ 


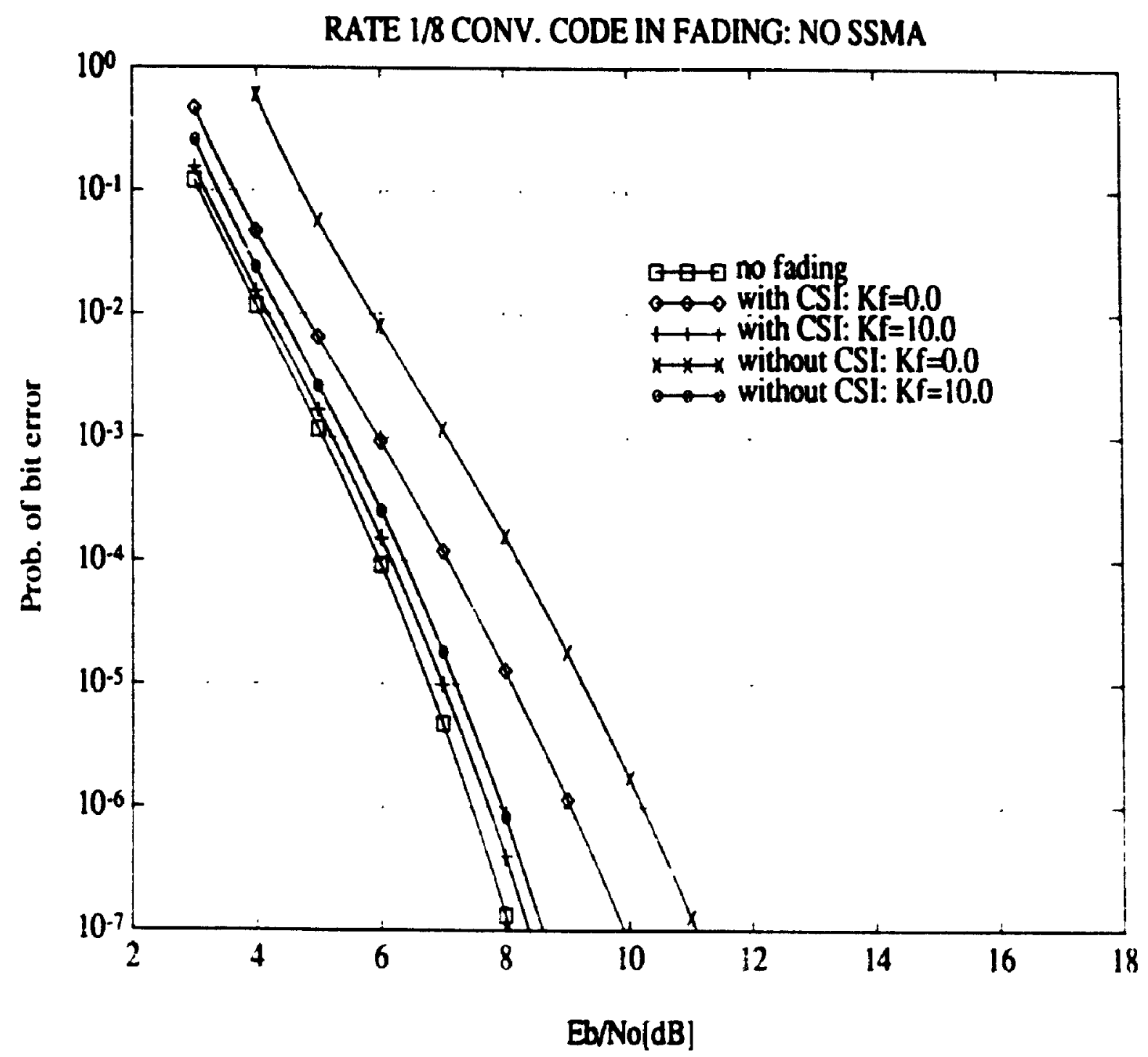

Figure 7.3: Performance of 4-state rate $1 / 8$ convolutional rode in Kirian fading, without SSMA 


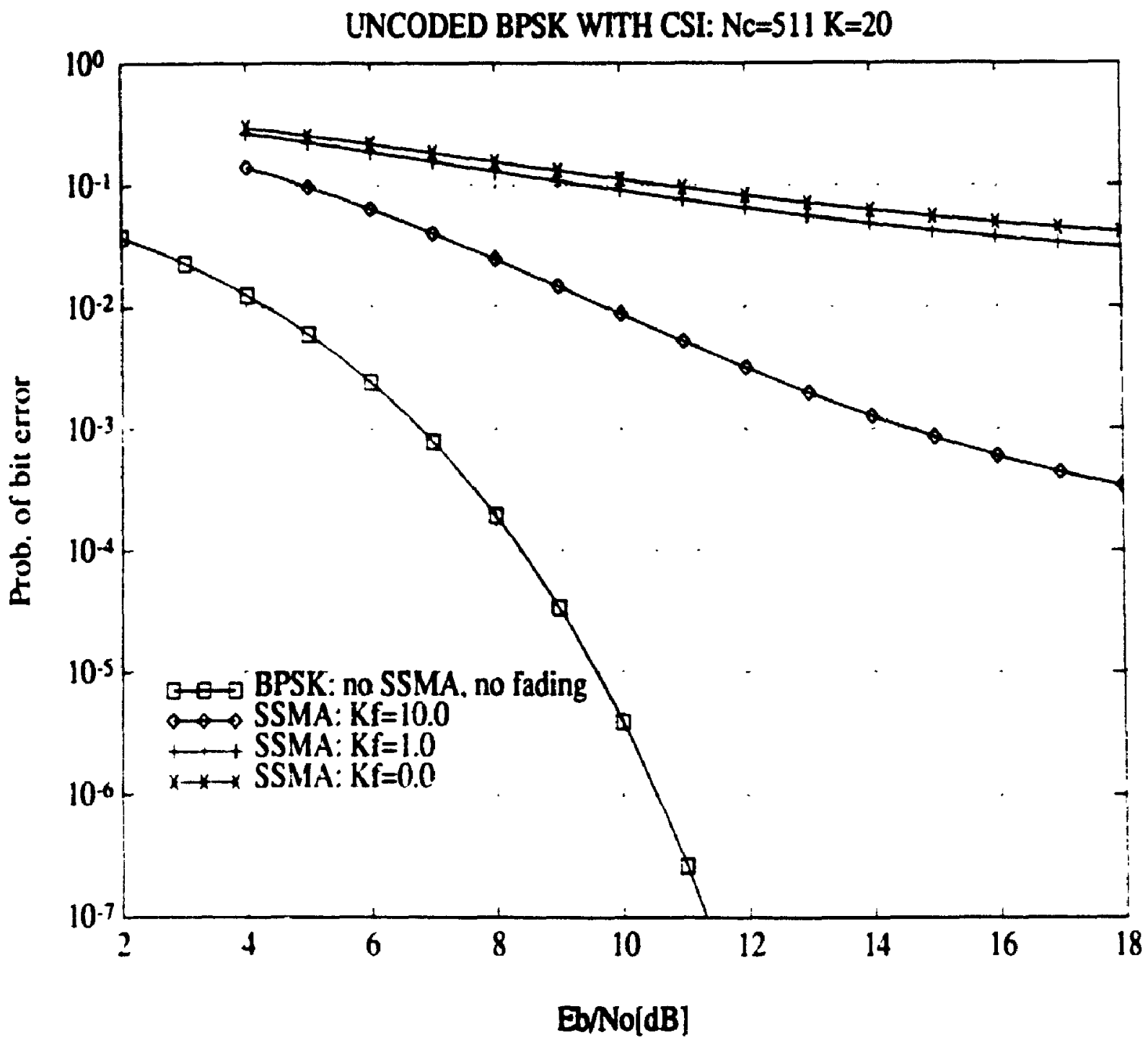

Pigure 7.1 : Performance of uncoded BPSK in Rician fading with CSI 


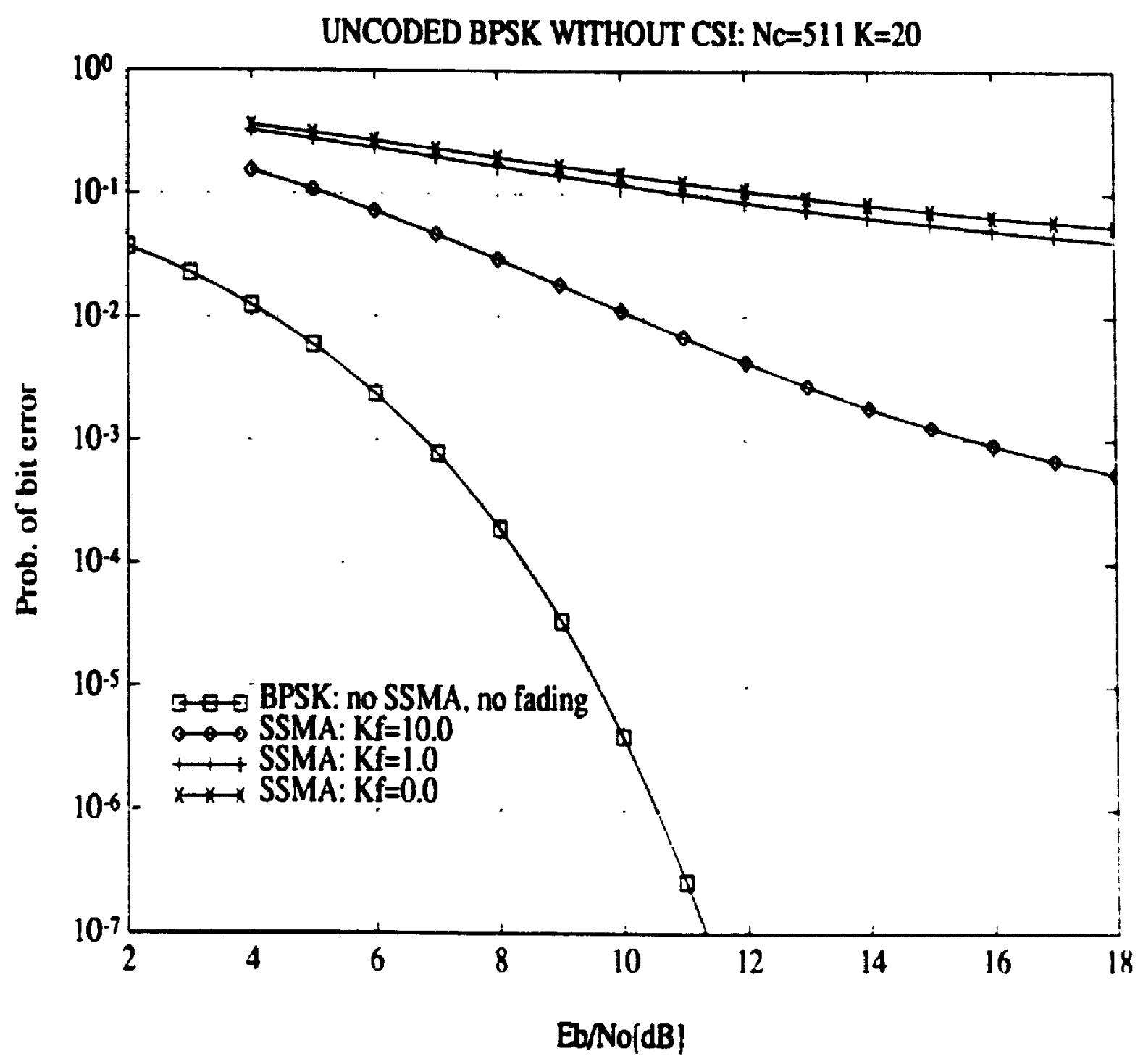

Figure i.5: Performance of uncoded BPSK in Ririan fading without ('SI 


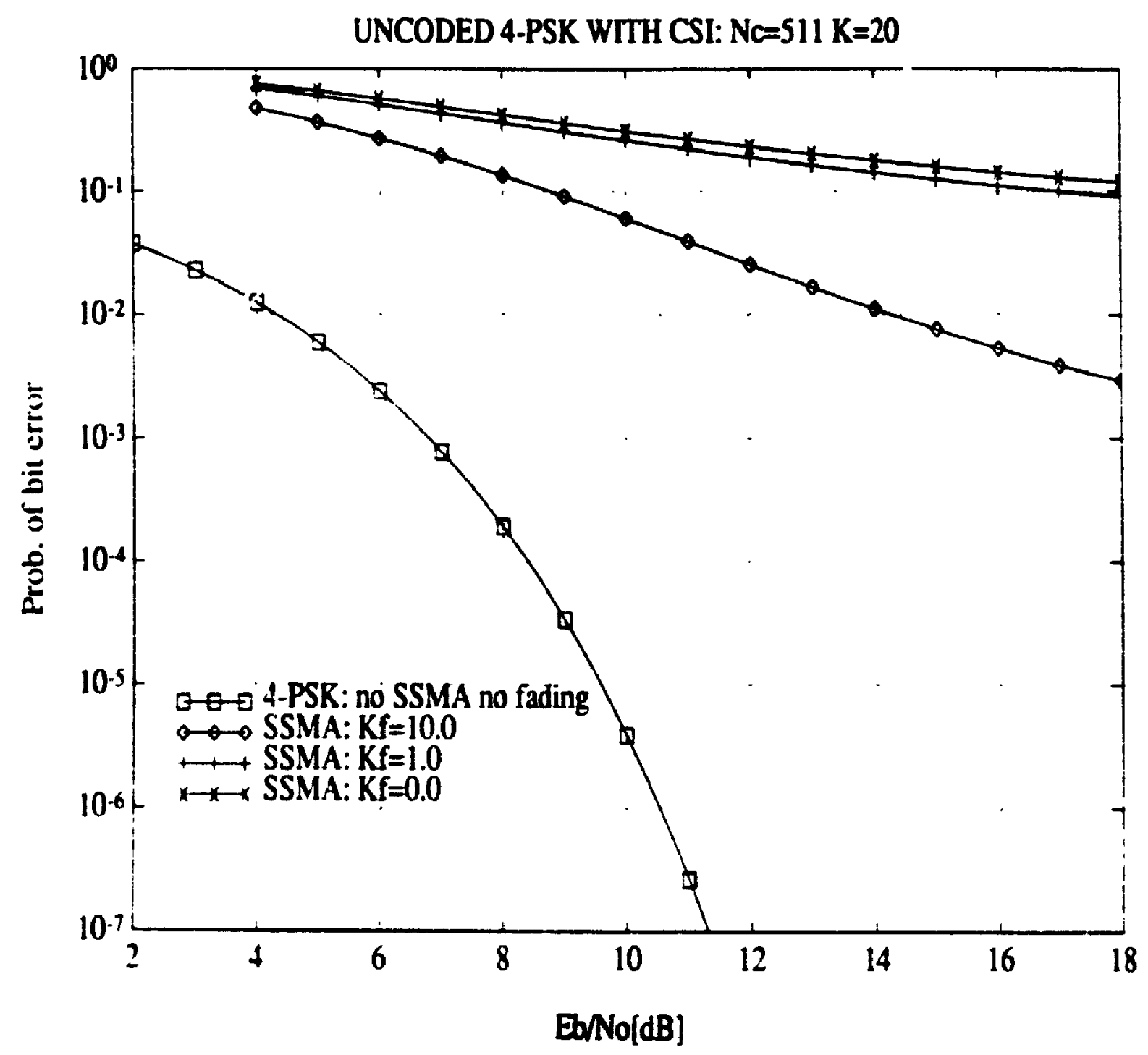

Figure 7.6: P'rformance of uncoded 4-PSK in Rician fading with CSI 


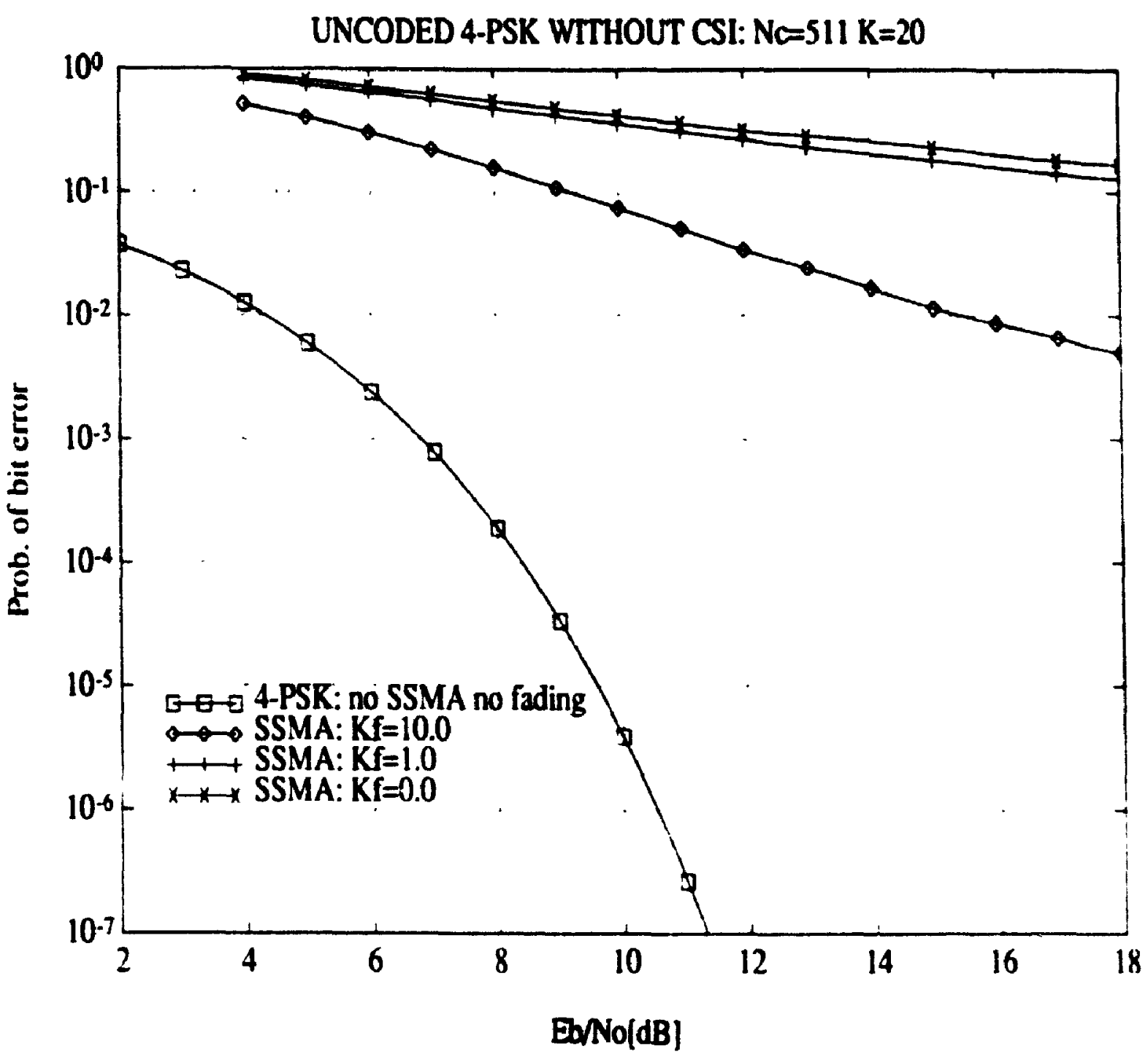

Figure 7.7: Performance of uncoded 4-PSK in Rician fading without ('SI 
curves consisting of the code performance with: no IUI or fading; IUI but no fading; Ilil and fading present with a Riciar fading parameter $K_{f}$ of 10.0, 1.0 and 0.0. All of the curves in which ICI is present assume a SSMA system with $k=20$ users and PN spreading sequences of length $N_{c}=511$. From these curves it is evident that the presence of a fading channel has a very detrimental effect on the BER performance of a trellis coled SSMA system. For example at a BER of $10^{-5}$, the 4-state 4-PSK code operating in a Rician fading channel at $K_{f}=10.0$ with CSI (recali that $K_{f}$ is the ratio of the direct line of sight signal component to the diffuse component), requires an $\frac{F_{b}}{N_{0}}$ of $10 \mathrm{~dB}$, ncarly $2.4 \mathrm{~dB}$ more than is needed when no fading is present. If no ( SSI is present. an additional $1 \mathrm{~dB}$ is required to attain a BER of $10^{-5}$. Furthermore when $k_{f}$ is derreased to 1.0 the BER curve tends toward saturation at a BER of approximately $10^{-4}$ for the case with CSI. At a BER of $10^{-3}$ (with CSI), the degradation in the fading channel is over $7.5 \mathrm{~dB}$ with respect to the performance of the rode having distortion due to AWGN and ILI aione. When no CSI is present, the degradation is almost $10.5 \mathrm{~dB}$ at a BER of $10^{-3}$ when Rician fading is added to the SSMA system.

The \&-PSK trellis codes exhibit at even sharper deg*adation in performance than do the A.PSK codes. in the presence of Rician fad. is This is due in part to the smaller minimum branch distance of the X-PS 1 . s.gnal constellation. When CSI is present the 4-itate 8 -PSk code BER curve saturates at a BER below $10^{-4}$ for $k_{f}=10.0$, whereas for $h_{f}$ values of 1.0 and 0.0 , the BER curves saturate at a BER above 10-2. If the system has no ('SI a vailable, the BER floor is increased as is illust rated in rigure 7.11 .

Increasing the complexity of the 8-PSK code to 16 states results in significant coding gains as is ilustrated in Figure 7.12 . When CSI is present a BER of $10^{-5}$ can now be altaincd at an $\frac{\ddot{z}_{0}}{N_{0}}$ of $11.8 \mathrm{~dB}$ for $K_{j}=10.0$, whereas the curves for $K_{j}$ valuss of 1.0 and 0.0 saturate at approximately $2 \times 10^{-3}$ and $6 \times 10^{-3}$ respectively. If no ('Sl is prosent. an additional $1 . j \mathrm{~dB}$ is required to attain a BER of $10^{-5}$ for 


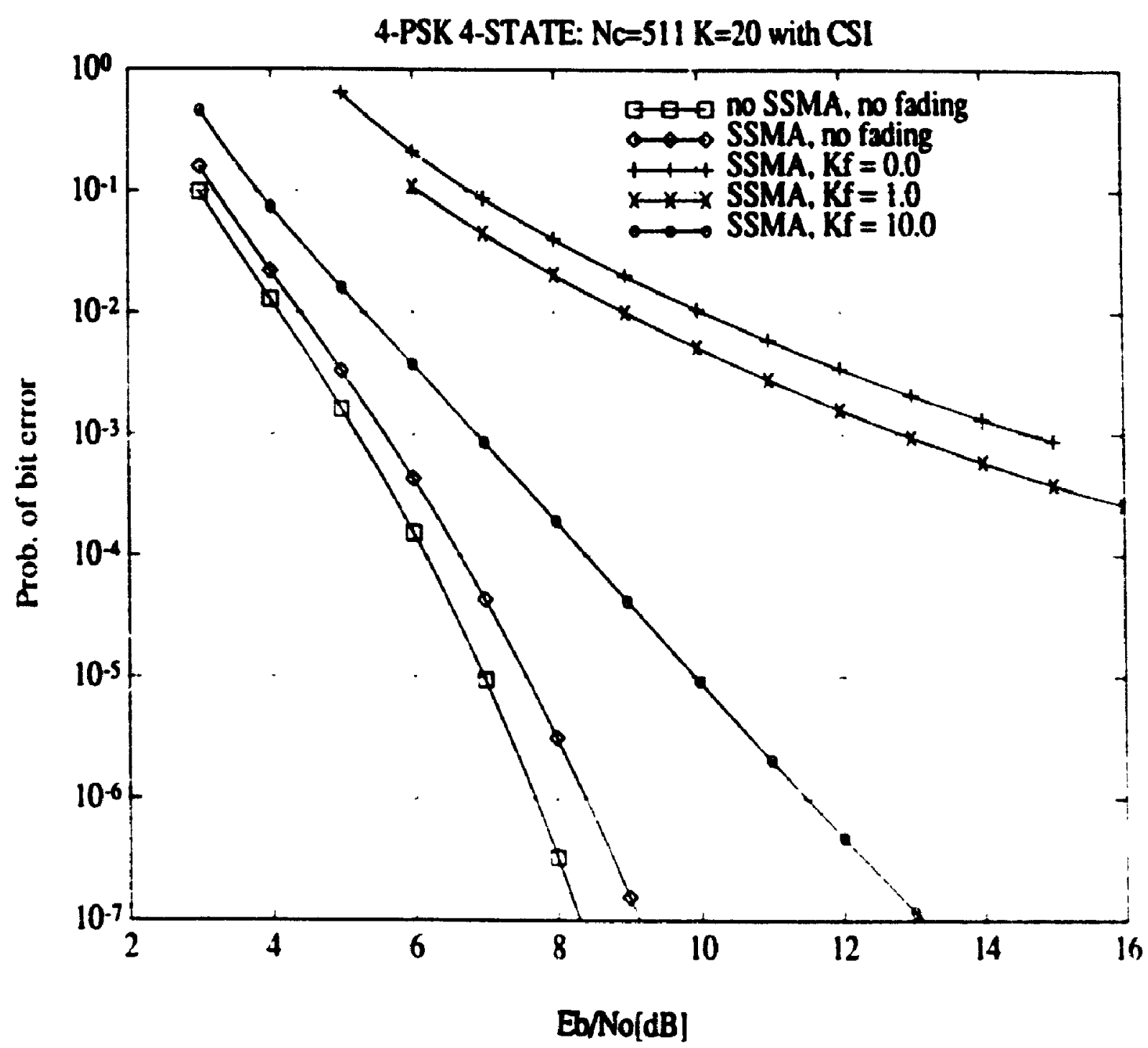

Figure 7.8: Performance of 4-PSK 4-state trellis code for $k=20$ usters in a Miciall fading channel with C.SI for $k_{f}=10.0 .1 .0$ and $0.0\left(N_{f}=.311\right)$. 


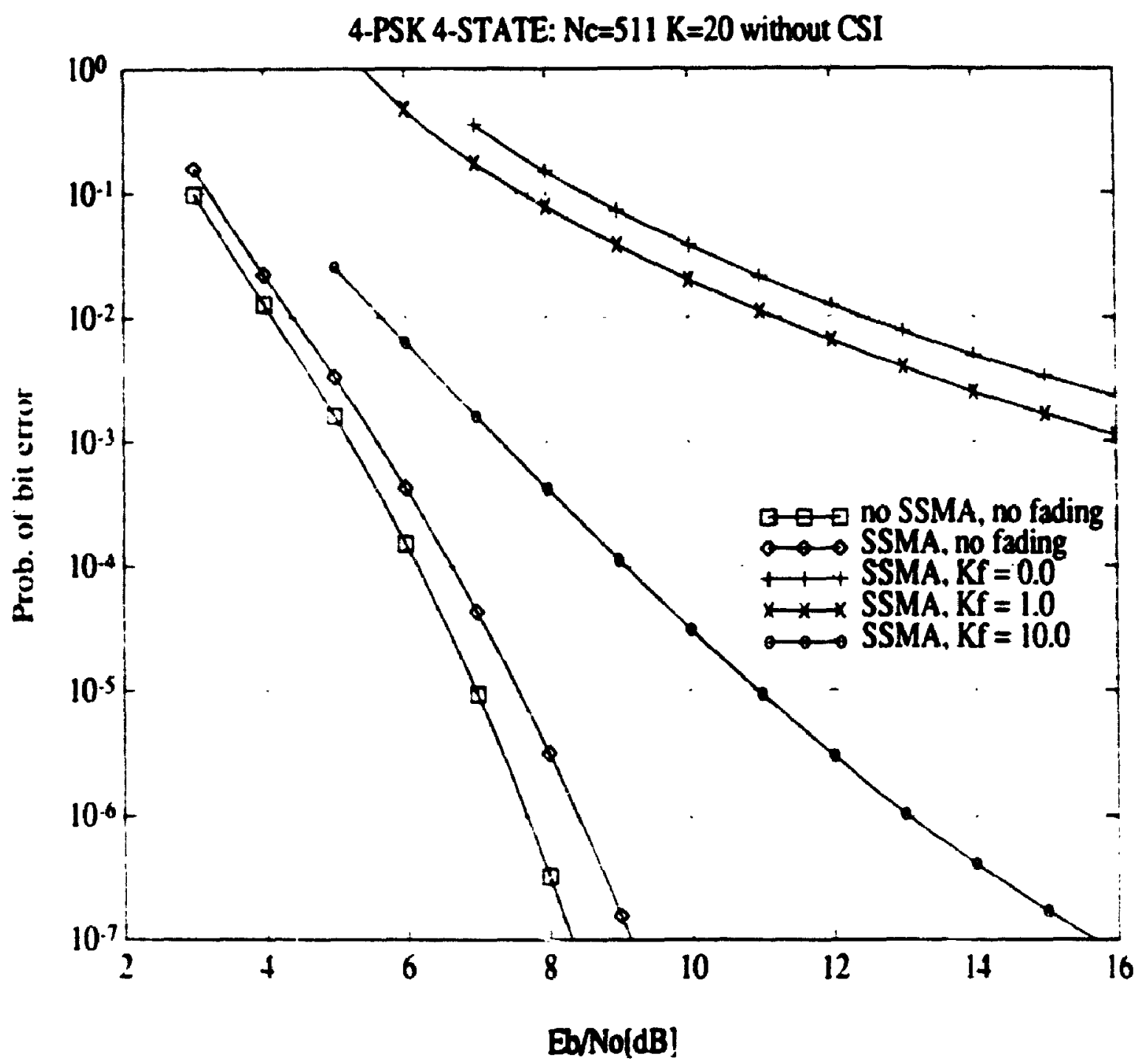

Figure 7.9: Performance of 4-PSK 4-state trellis code for $k=20$ users in a Rician fading channel with no ('SI for $K_{f}=10.0,1.0$ and $0.0\left(X_{i}=511\right)$. 


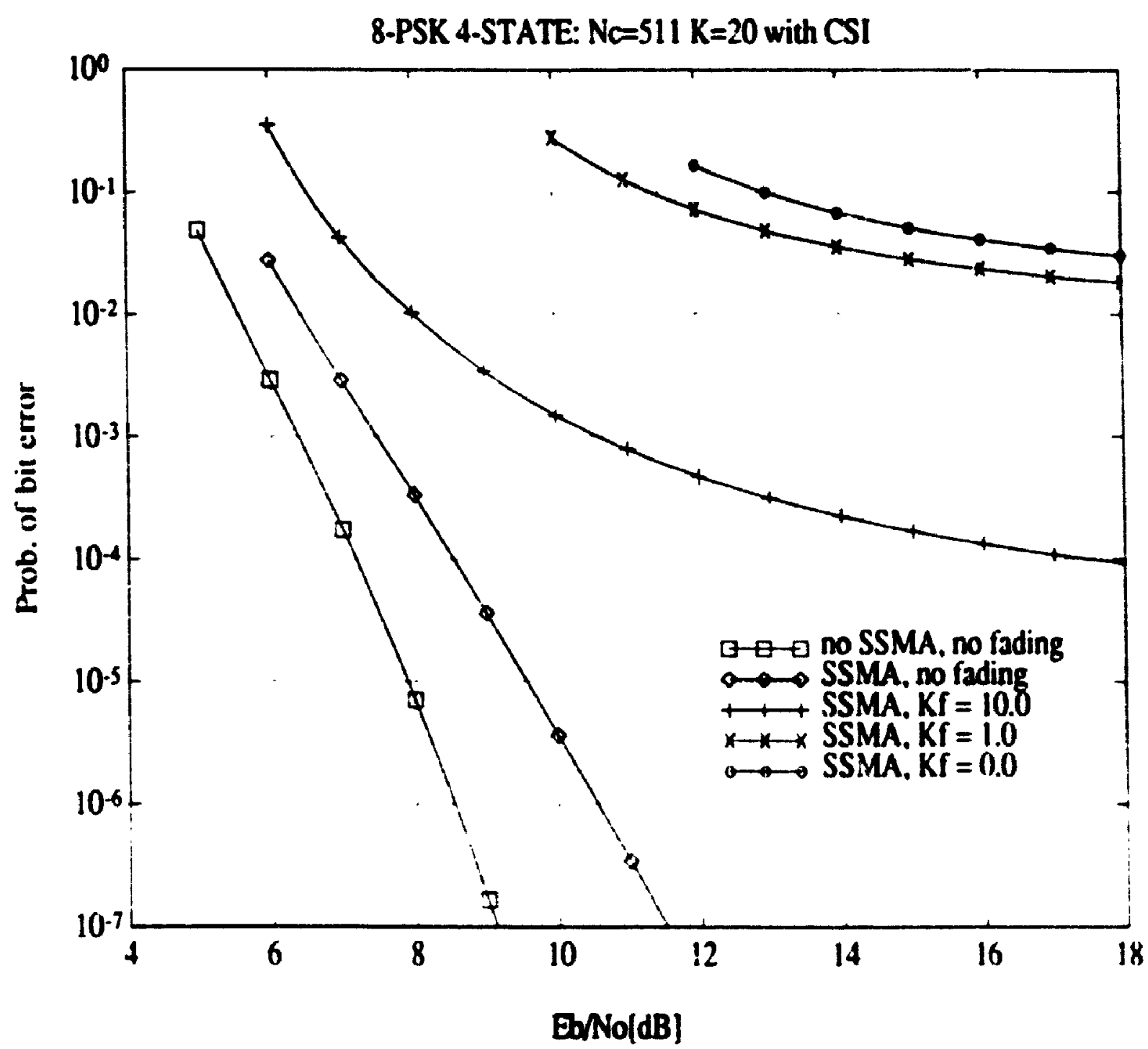

Figure 7.10: Performance of 8 -PSh 4-state $t$ rellis cule for $k=20$ users in a Riciall fading channel with (SI for $\left.K_{f}=10.0 .1 .0 \mathrm{and} 0.0(X)=.11\right)$. 


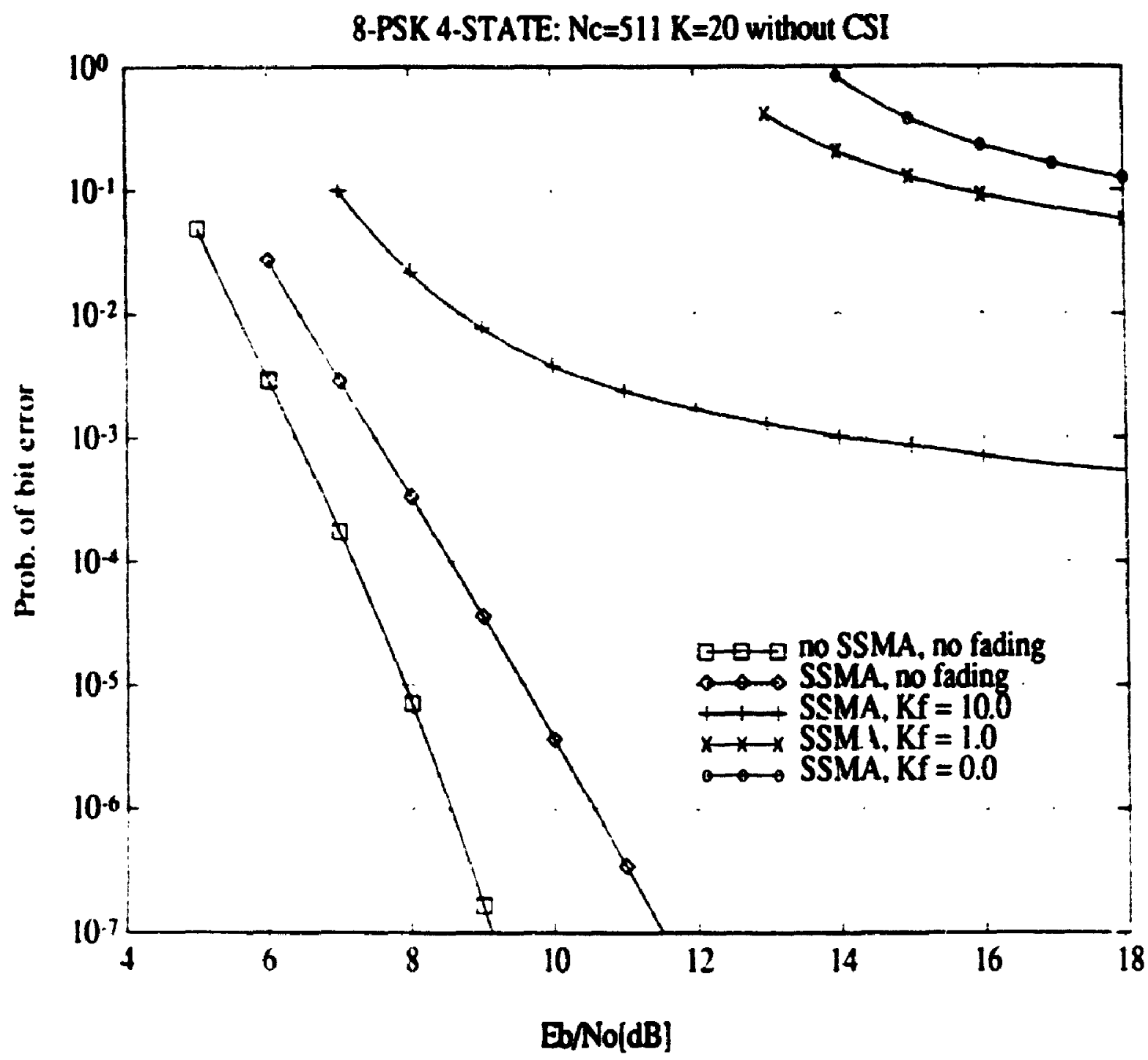

Figure 7.11: Performance of R-PSK 4-state trellis code for $K=20$ users in a Rician fading chanmel with no ('SI for $k_{f}=10.0 .1 .0$ and $0.0\left(V_{c}=511\right)$. 


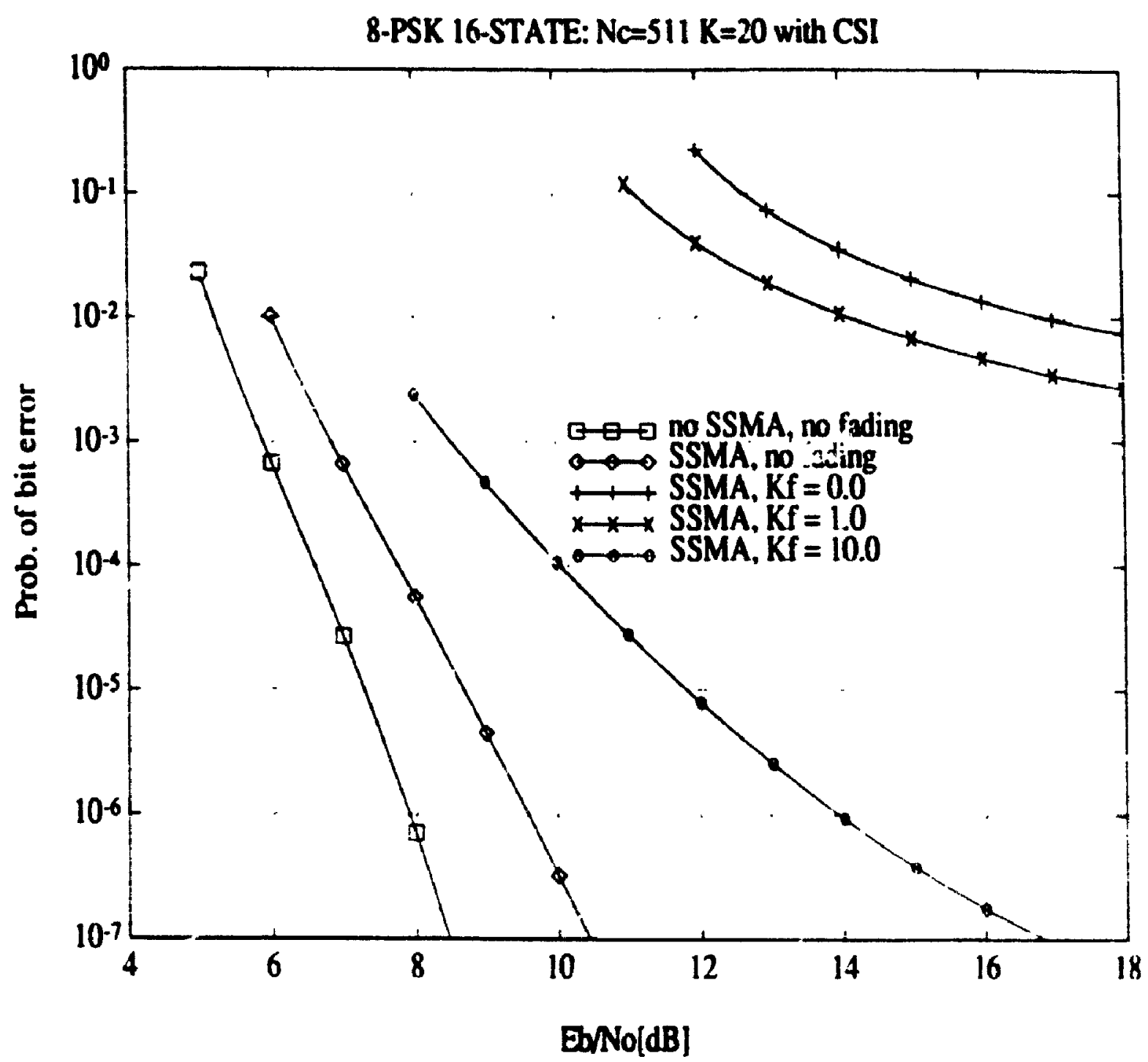

Figure 7.12: Performance of 8-PSK 16-state trellis corde for $k=20$ use-rs in a Rician fading channel with CSI for $K_{f}=10.0$, and $1.0\left(N_{r}=i 11\right)$. 


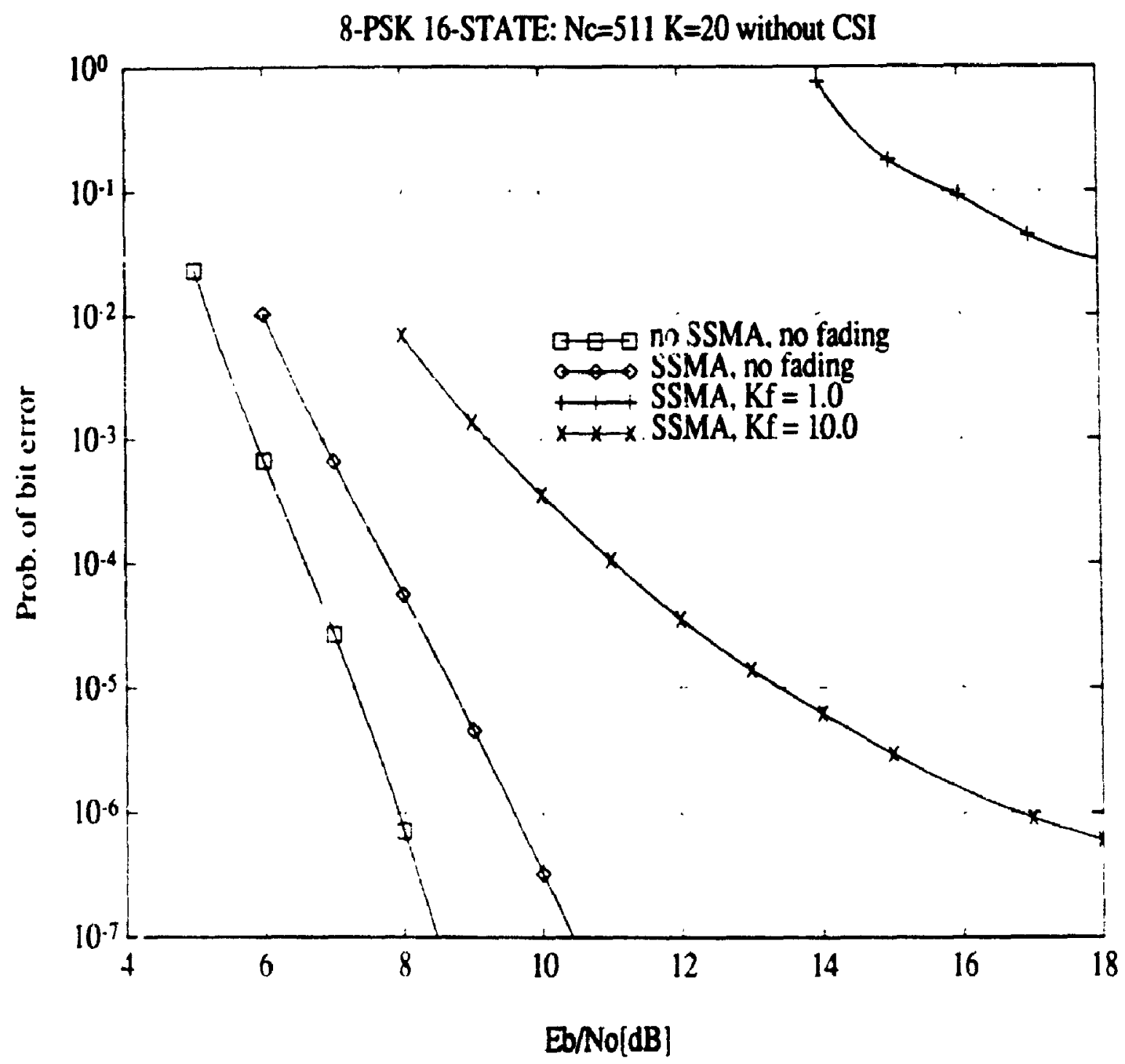

Figure 7.13: Performance of $\mathrm{X}$-PSK 16-state trellis code for $\mathrm{K}=20$ users in Rician iading with no ('SI for $K_{f}=10.0 .1 .0$ and $0.0\left(N_{r}=511\right.$ ). 
$K_{f}=10.0$. In comparison to the 4-PSK trellis code. it can be seen that the 8-Psk code is inferior, even when compared on a basis of equal throughput. For example comparing the 4-state codes at a BER of $10^{-4}$. the 4-PSK code is ti.idls superior to the 8-PSK code even after accounting for the $3 \mathrm{~dB}$ required to attain the same throughput.

\subsection{Convolutional Codes with SSMA in Rician Fading}

The performance of 4 and 16 state rate $\frac{1}{2}, \frac{1}{3}$ and $\frac{2}{2}$ convolutional codes in a SSMA environment. with and without CSI are plotted in Figures 7.14107 .25 . As in the previous section. the code performance is plotted for: no III or fading; III with no fading; ILI with Rician fading rith $k$, values of 10.0 . 1.1 and 0.0 . As in the case of

the results for the AWGN channel of (liapter 5, the rate $\frac{1}{2}$ codes were amploverl in conjunction with Gold spreading codes of length $N=255$ chips, whereas the rate $\frac{1}{8}$ and $\frac{2}{2}$ codes were cmployed in conjunction with ciold spreading code: of langth $V_{c}=63$. All of the codes were assumed to be operating in a SSMA ystente simplioving $K=20$ users.

The same general performance trends that were cheserved for the purformanes of the trellis codes in a fading channel can be observer for the preformance of the convolutional codes in the fading channel. As is expected the systeme ampleyinge channel state information are superior to those without ('SI. Howerer the saturating

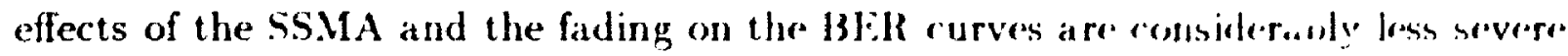
than was observed for the trellis codes. For example comparing the 4-utate rate

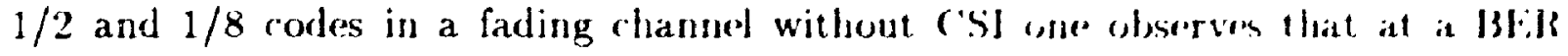
of $10^{-5}$ that the rate $1 / 8$ convolutional code is about 1 d B superior te the rate $1 / 2$ convolutional code for $K_{f}=10.0$. As $K_{j}$ is decreased 10 l. (1) the gain of the rite $1 / 8$ code over the rate $1 / 2$ code increases to $5.4 \mathrm{dth}$. 
RATE 1/2 4-STATE CONV. CODE: Nc $=255 \mathrm{~K}=20$ with CSI

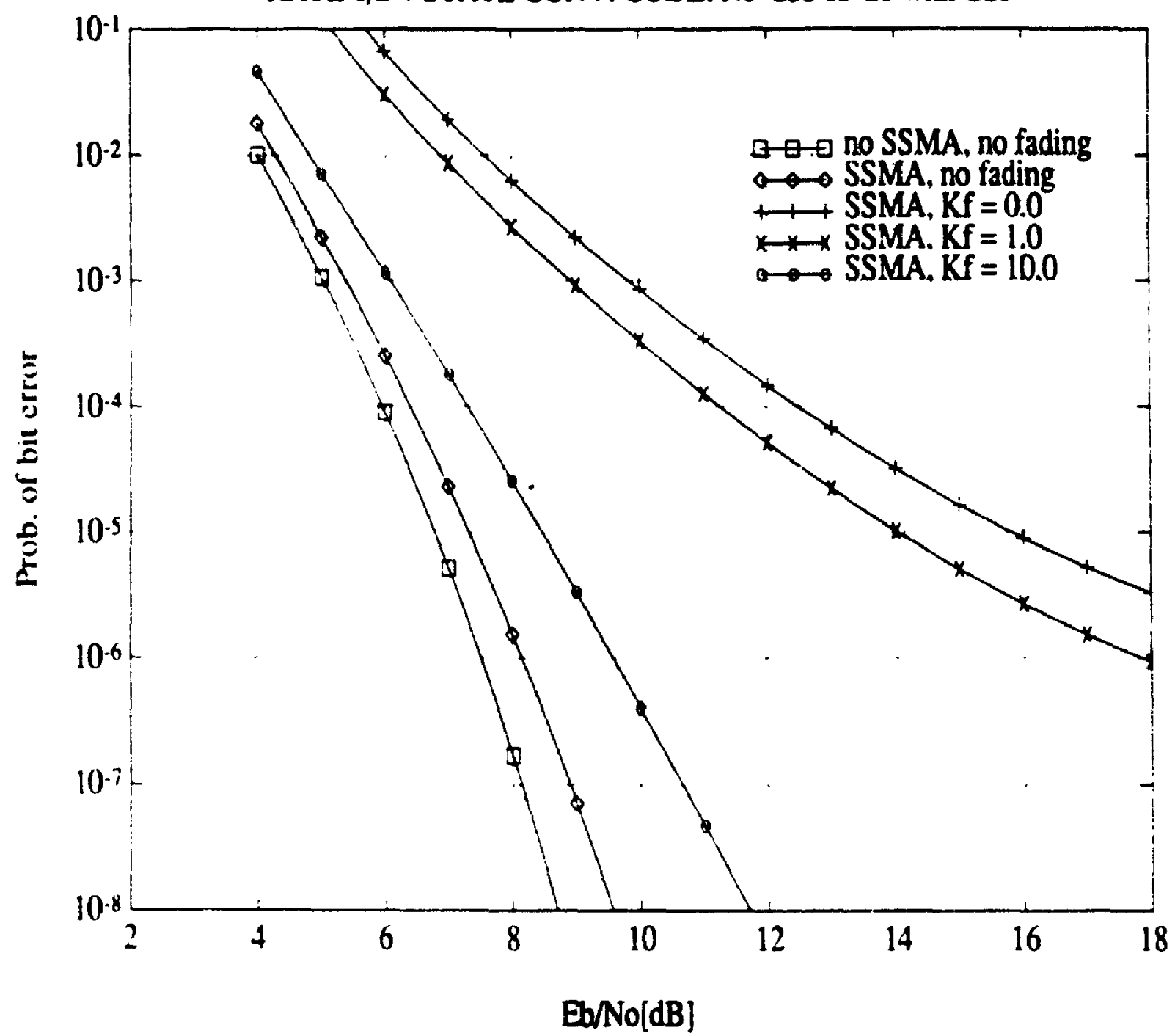

Figure 7.14: Performance of rate $1 / 2$ 4-state convolutional code for $\mathrm{K}=20$ users in Rician fading wilh ('Sl for $h_{f}=10.0,1.0$ and $0.0\left(N_{i}=255\right)$ ). 


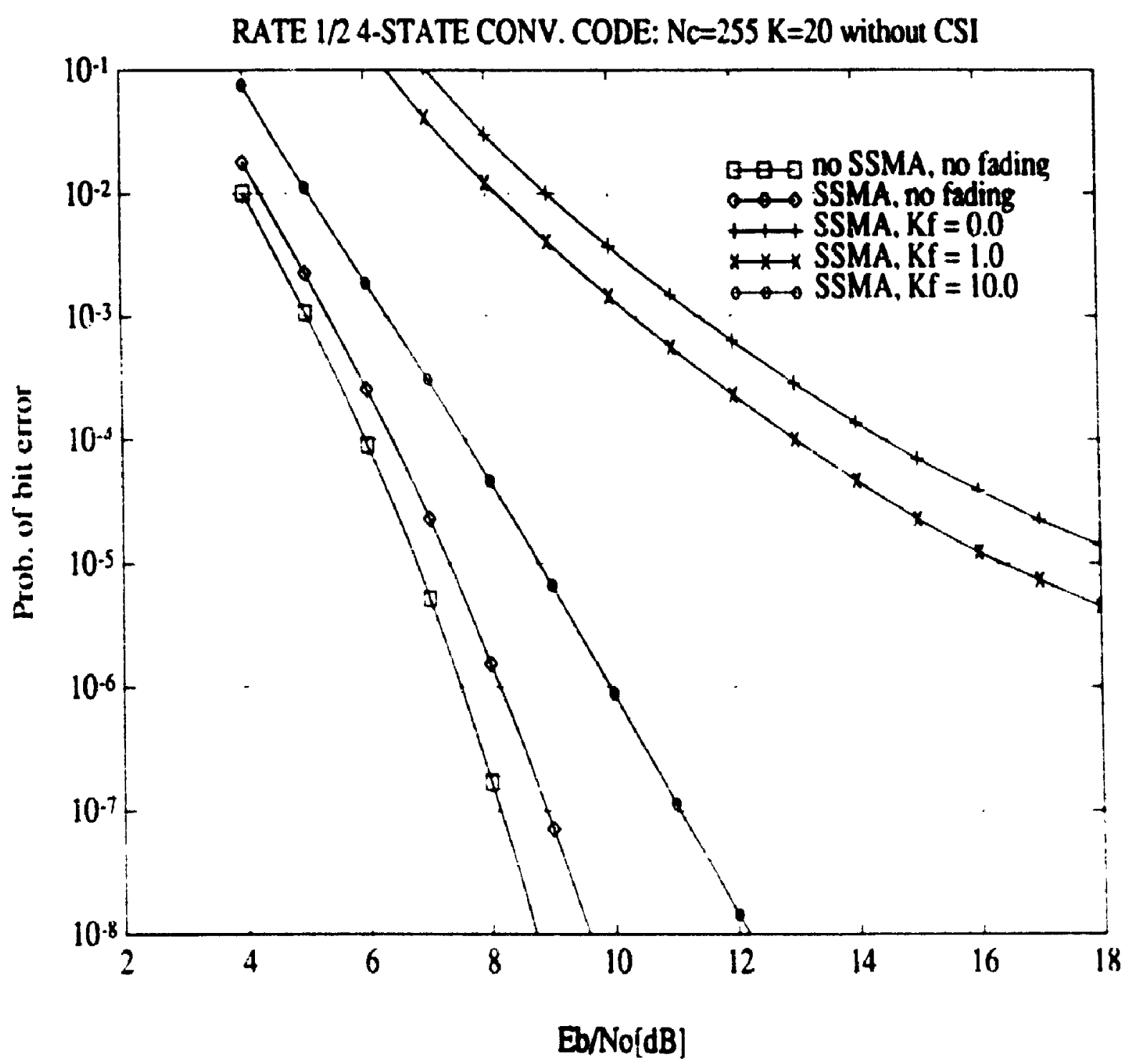

Figure 7.15: Performance of rate $1 / 2$ 4-state convolutional code for $k=20$ users in Rician fading without CSI for $K_{\circ}=10.0,1.0$ and $0.0\left(N_{r}=25 \%\right)$. 


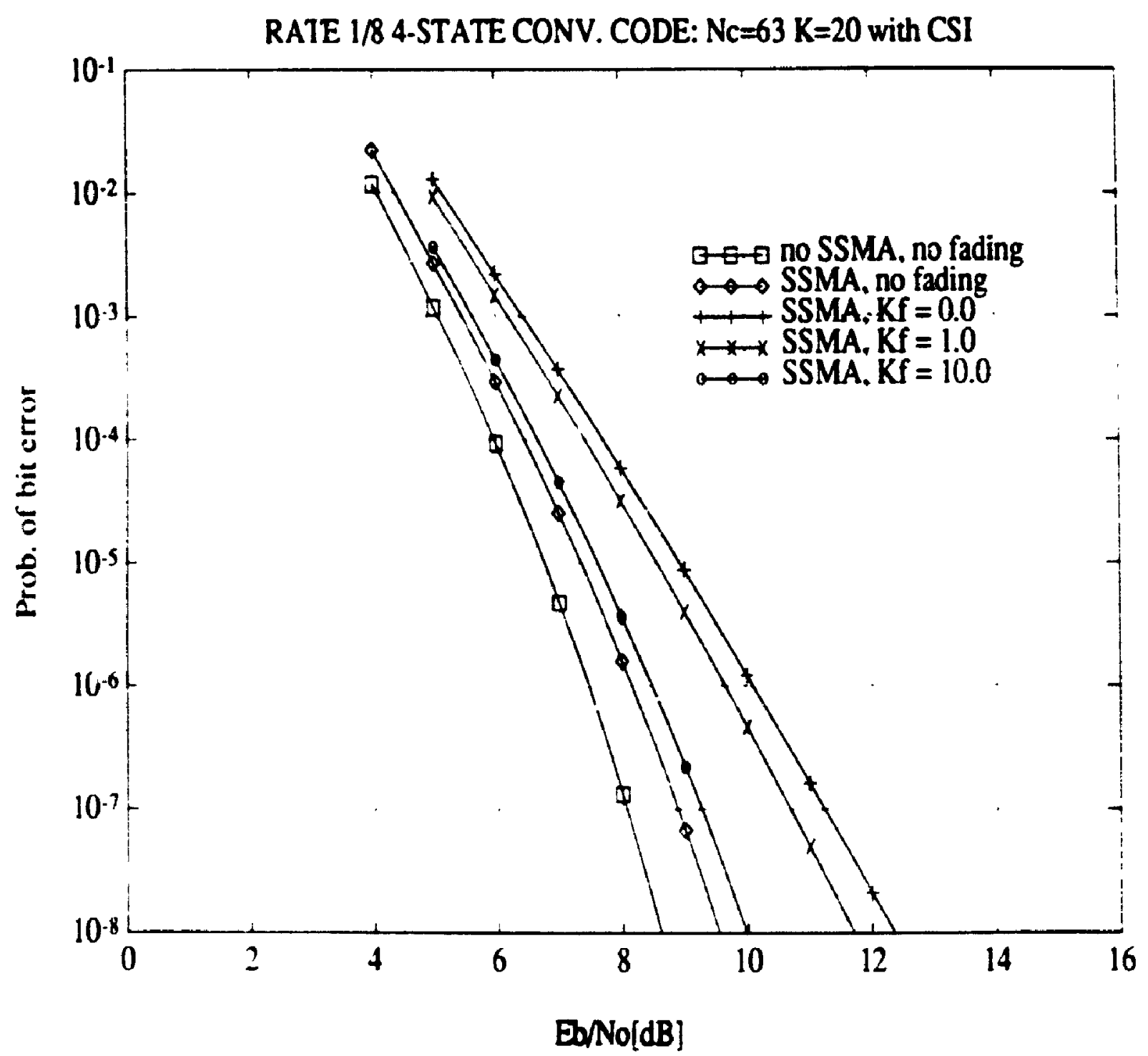

Figure 7.16 : Performance of rate $1 / 8$ 4-state convolutional code for $\mathrm{K}=20$ users in Rician fading with ('S! for $K_{f}=10.0 .1 .0$ and $0.0\left(N_{c}=63\right)$. 


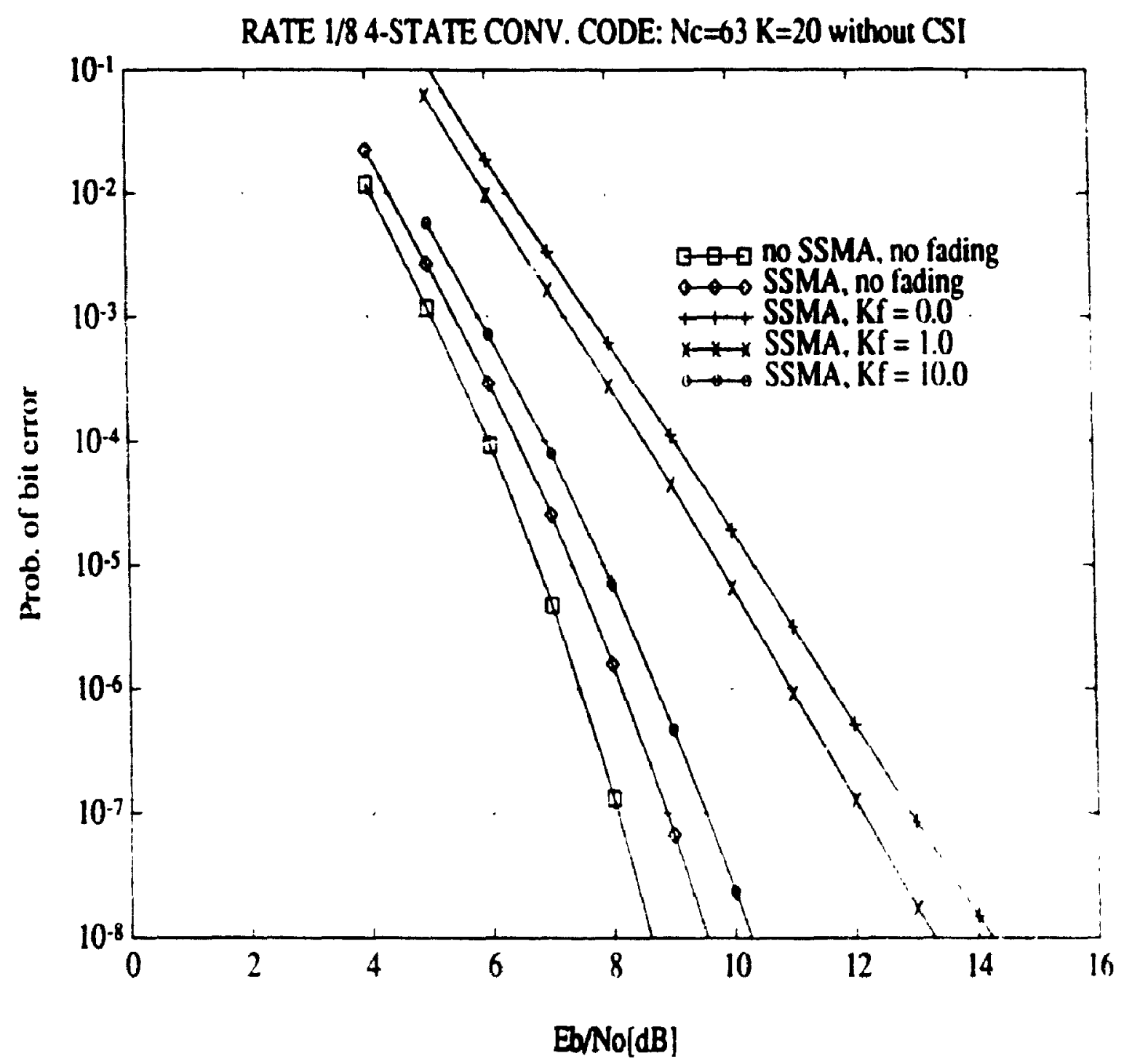

Figure 7.17: Performance of rate 1/8 4-state convolutional code for $k=20$ use.5s in Rician fading without C.SI for $k_{j}=10.0 .1 .0$ and 0.1$)\left(V_{r}=633\right)$. 


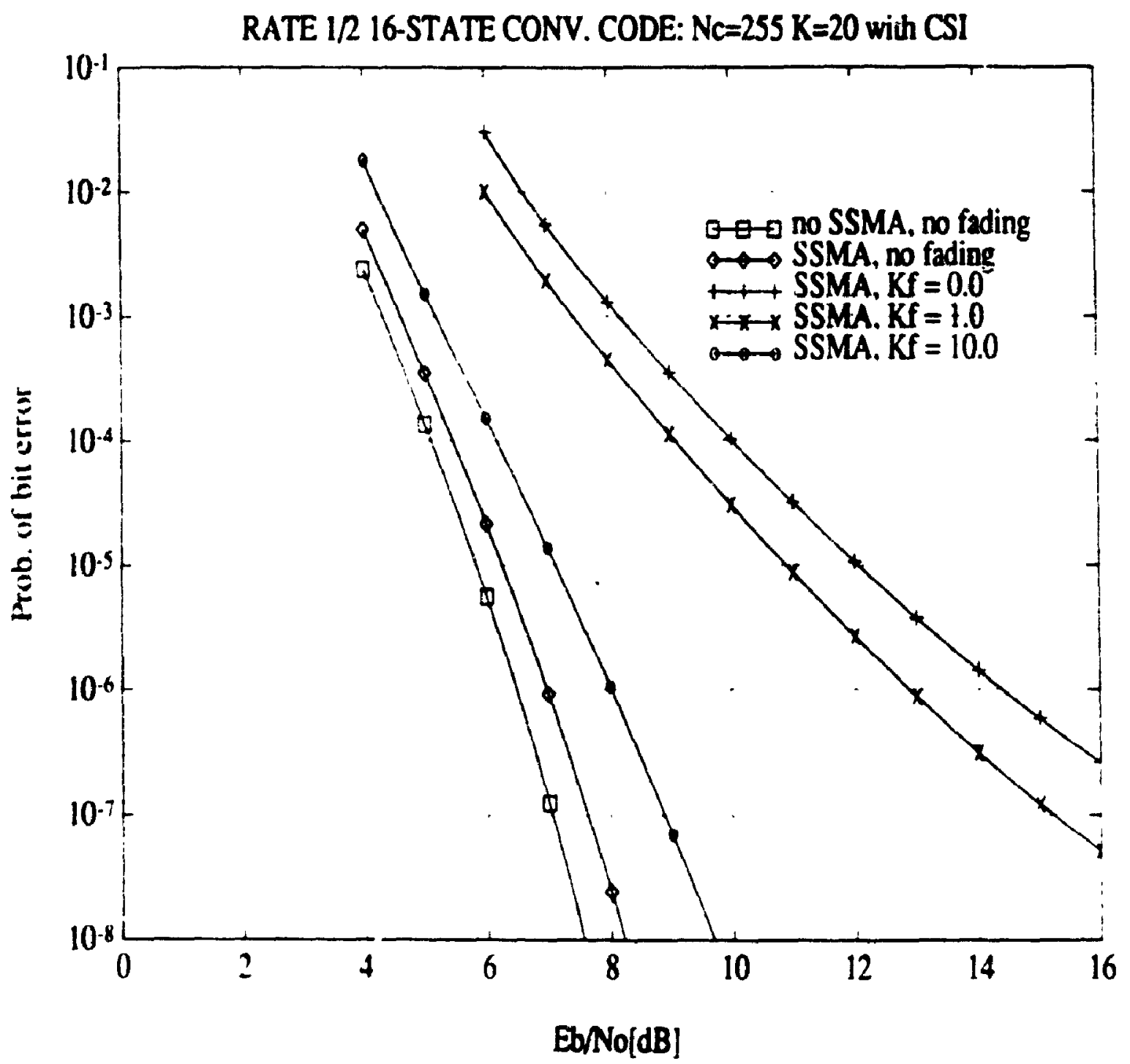

Figure 7.18 : Performance of rate $1 / 216$-state convolutional code for $\mathrm{K}=20$ users in Rician fading with ('Sl for $K_{f}=10.0,1.0$ and $0.0\left(N_{c}=255\right)$. 


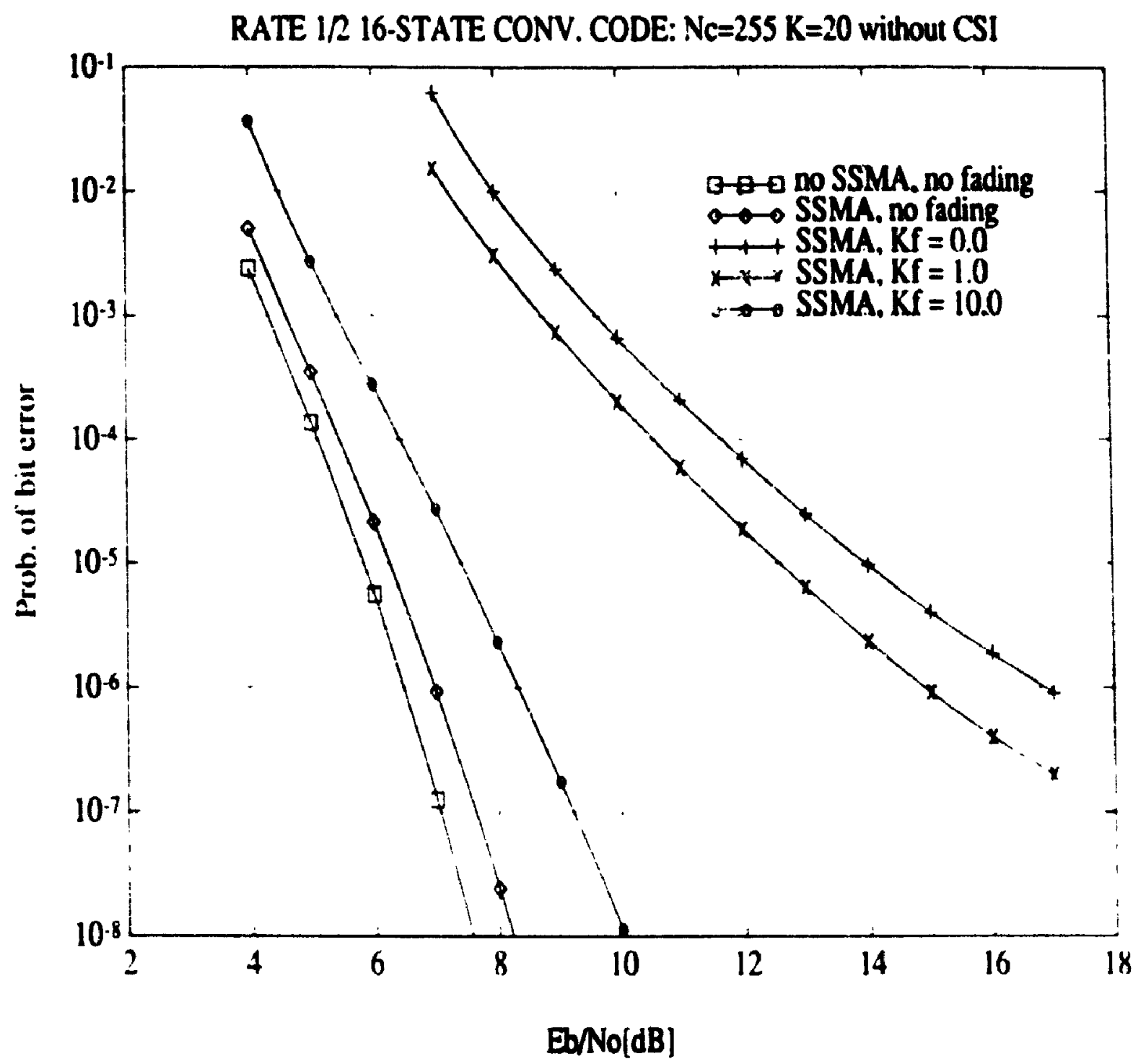

Figure 7.19: Performance of rate 1/2 16-state convolutional code for $k: 20$ use-rs in Ririan fading without CSI for $K_{f}=10.0,1.0$ and $0.0\left(X_{f}=2.5 .5\right)$. 


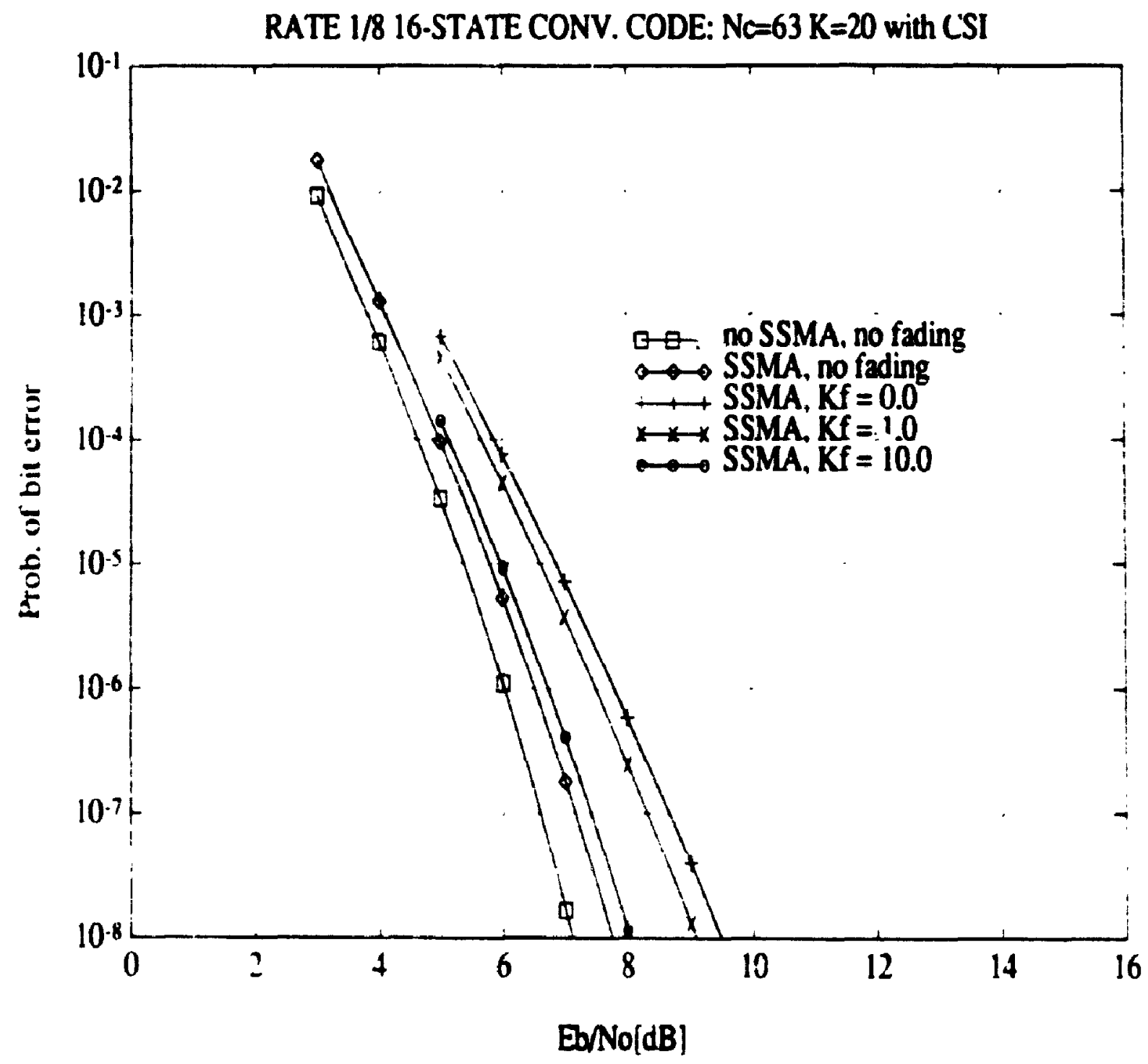

Figure 7.20: Performance of rate 1/8 16-state convolutional code for $k=20$ users in Rician fading with ('SI for $K_{f}=10.0 .1 .0$ and $0.0\left(N_{c}=63\right)$. 


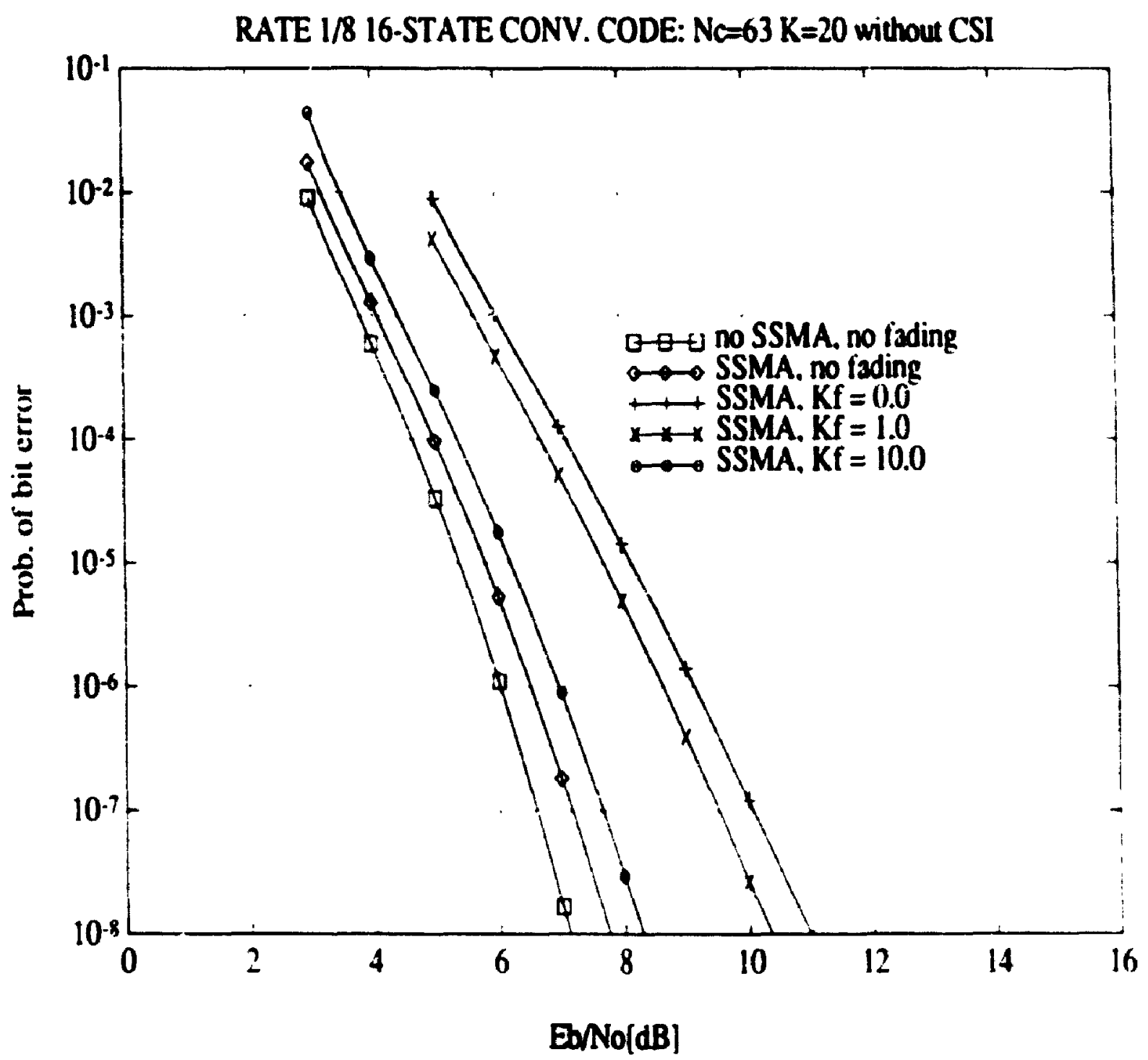

Figure 7.21: Performance of rate $1 / 816$-state convolutional code for $K=20$ use-rs in Rician fading without CSI for $K_{f}=10.0 .1 .0$ and $0.0\left(N_{r}=(; .3)\right.$. 


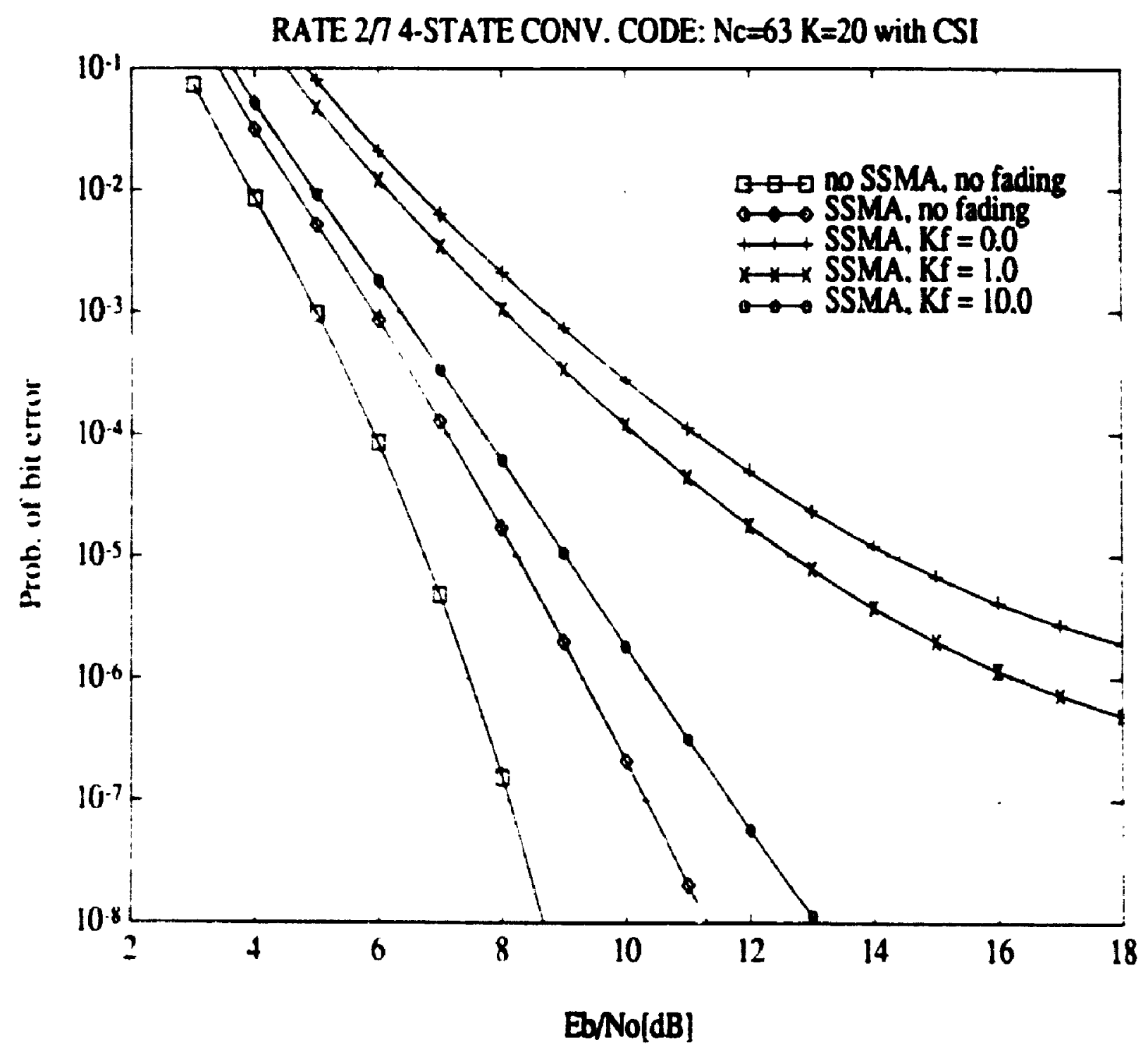

Figure $7: 2:$ : Performance of rate $2 / \hat{\imath}$ 4-state convolutional code for $K=20$ users in Rician fading with ('SI for $K_{f}=10.0 .1 .0$ and $0.0\left(. V_{c}=63\right)$. 


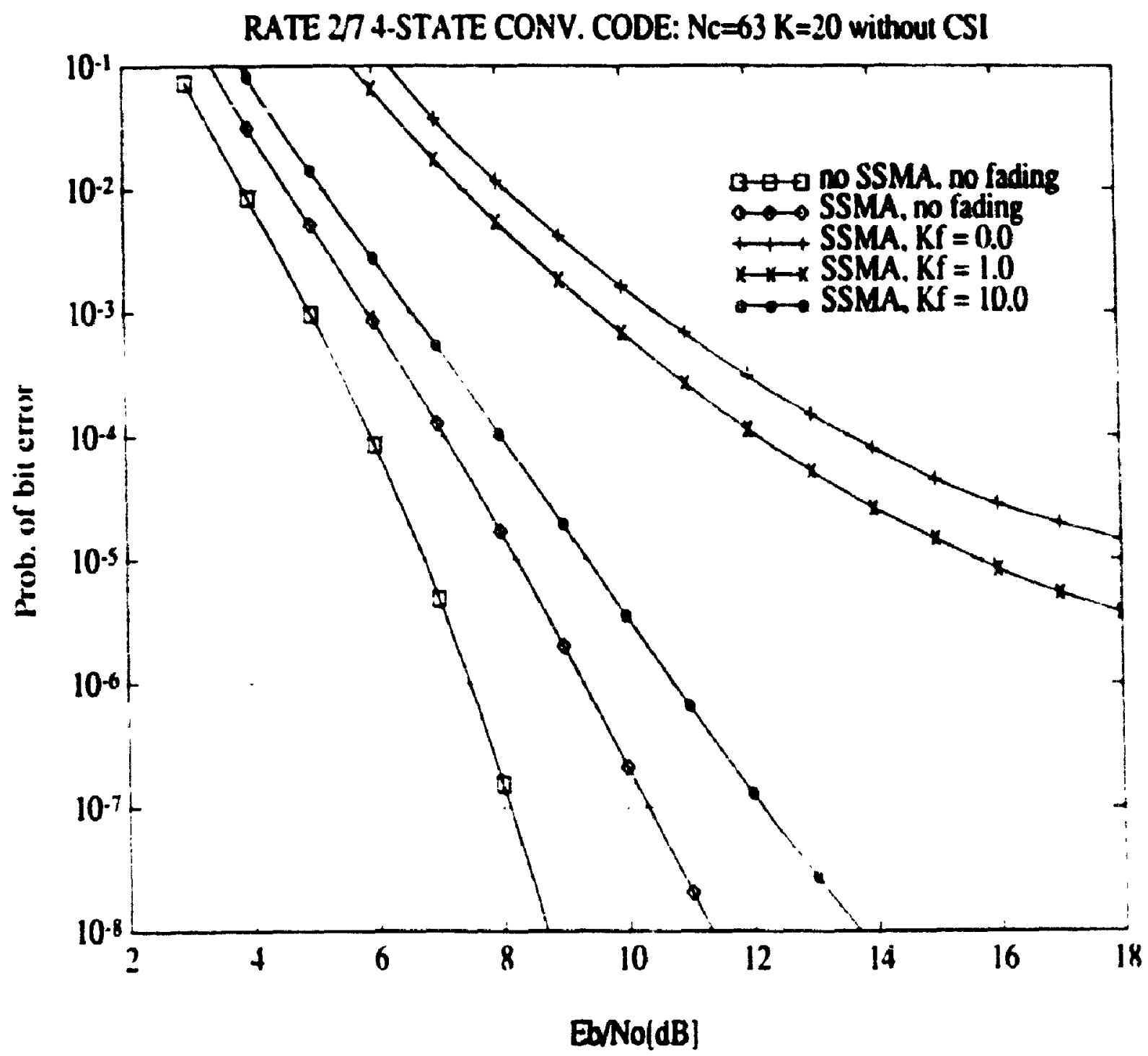

Figure 7.23: Performance of rate 2/ $i$ 4-state convolutional code for $K=2(1)$ users in Rician fading without (CSI for $K_{j}=10.0,1.0$ and $0.0\left(N_{j}=1.3\right)$. 


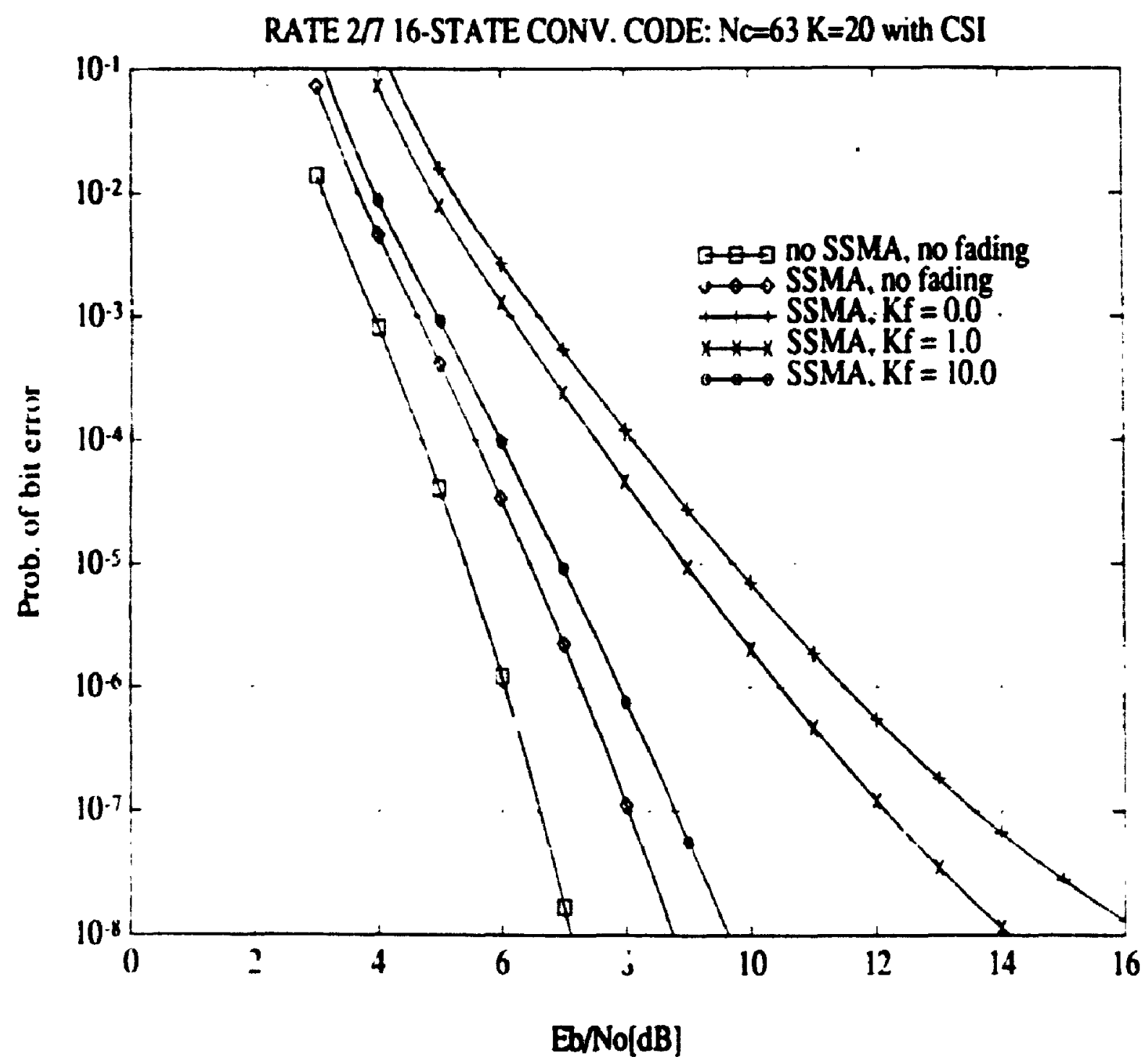

Figure $7: 2$ 1: Performance of rate $2 / 7$ 16-state convolutional code for $K=20$ users in Rician fading with ('SI for $k_{f}=10.0 .1 .0$ and $0.0\left(N_{c}=63\right)$. 


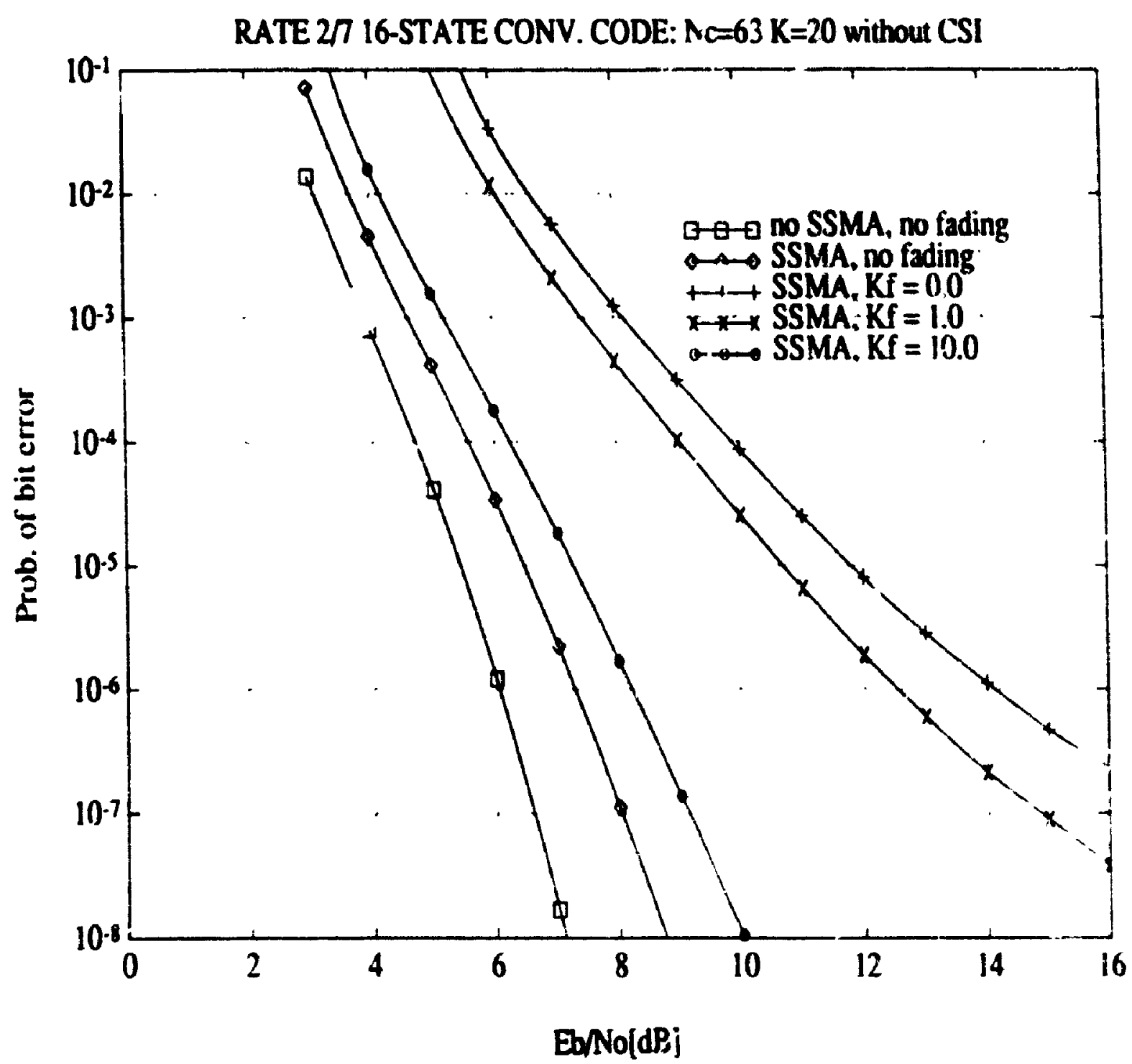

Figure 7.25: Performance of rate $2 / 7$ 16-state convoluticial cisle for $k=20$ is.rs in Rician fading without CSI for $K_{f}=10.0,1.0$ and $0.0(N$. i3). 

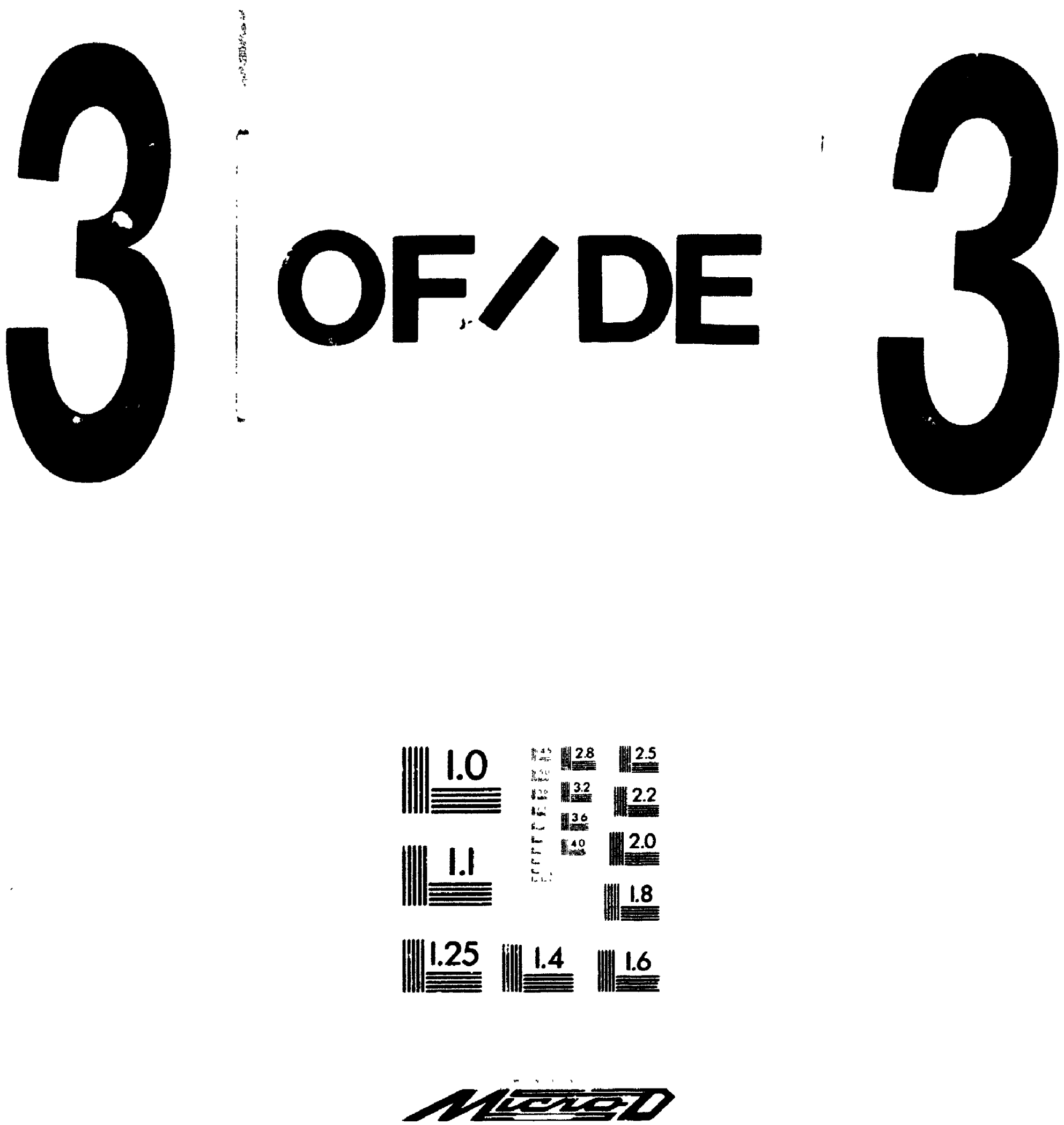
Similar gains in performance between the two codes can be observed under conditions of no CSI. Comparing the BER performance of the rate $1 / 2$ and $1 / \varepsilon$ 4-state: convolutional codes to the 4-state 4-PSK code, one can observe that fo: $\kappa_{f}=10.0$. the rate $1 / 2$ convolutional code provides an additional gain of $1.5 \mathrm{~dB}$ over the 4-PSK code, whereas the rate $1 / 8$ code provides an additional gain of $2.4 \mathrm{~dB}$ over that of the 4-PSK code.

Examining the 16-state rate $1 / 2$ and $1 / 8$ convolutional code peiformance in Fig. ures 7.18 to 7.21 it is again observed that the rate $1 / 8$ code provides superior performance over the rate $1 / 2$ code, with and without CSI. In particular, note that the rate $1 / 8$ exhibits no saturation effect, even for $K_{f}=0.0$, whereas the rate $1 / 2$ code continues to exhibit a trend towards saturation. both with and without ('SI. For example, for $K_{f}=0.0$, an $\frac{E_{b}}{N_{0}}$ of $6.9 \mathrm{~dB}$ is required by the rate $1 / 8$ convolutional code versus the $12.0 \mathrm{~dB}$ required by the rate $1 / 2$ convolutional code.

The performance of the rate $2 / 74$ and 16 state convolutional codes are illustrated in Figures 7.22 to 7.25 . At a BER of $10^{-5}$ with CSI and $K_{J}=10.0$, the 4 -state corde. requires an $\frac{E_{b}}{N_{0}}$ of $9.1 \mathrm{~dB}, 0.8 \mathrm{~dB}$ more than is required to achieve the same BER when no fading is present. Decreasing $K_{f}$ to 1.0 and 0.0 results in a required $\frac{E_{n}}{N_{n}}$ on $12.7 \mathrm{dl}$ and $14.3 \mathrm{~dB}$ respectively to achieve a BER of $10^{-5}$. Similar results are observed for the case in which no CSI is present. For $K_{f}=10.0$ the change in performance from the corresponding case with CSI is approximately a 0.3dB. Lowever for $k$, values of 1.0 or 0.0 the degradation increases to $3-4 \mathrm{~dB}$, depending on the BER desired.

The 16 -state rate $2 / 7$ code exhibits a significant increase in performance over the 4-state code. With CSI present. a BER of $10^{-5}$ can be achieved with an $\frac{v_{6}}{v_{0}}$ of $7.0 \mathrm{~dB}, 9.0 \mathrm{~dB}$ and $5.8 \mathrm{~dB}$ for $K_{f}$ values of 10.0 .1 .0 and 0.0 respectively. For $K_{f}=0.0$ this is an additional coding gain of $4.5 \mathrm{~dB}$ over the corresponding curve for the 4 . state code (i.e. the trend towards saturation is less severe for the higher complexity codec). When no CSI is present the additional roding gain of the 16 -state rate: $2 / 7$ convolutional code over the 4 -state code is even greater. For example for $k_{j}=0.0$ 
there is an $8.2 \mathrm{~dB}$ difference at a BER of $10^{-5}$.

As was the case in the 4 WGN channel, the rate $2 / 7$ convolutional code provides significant gains over the corresponding rate $2 / 3$ trellis coded SSMA system. Specifically, for $K_{f}=10.0$ with CSI the rate $2 / 7$ 16-state convolutional code provides an additional $4.8 \mathrm{~dB}$ of gain than does the 16 -state rate $2 / 3$ trellis code, whereas this increase in gain is $6.1 \mathrm{~dB}$ for $K_{f}=10.0$ and no CSI.

In order to provide an easier comparison of the performance of the various trellis and convolutional codes that have been investigated, Table 7.1 lists the $\frac{E_{b}}{N_{0}}$ required for a BER of $10^{-5}$ for cach of the codes investigated.

\subsection{Gaussian Approximation to IUI in Rician Fading}

The performance of certain of the previously studied trellis and convolutional codes assuming that the IUI can be modelled as a Gaussian random variable is presented below. As was the case for performance in just an AWGN channel, the use of the Gaussian Assumption leads to overly optimistic performance predictions as compared to the Chernoff bound technique. Representative cases of performance with CSI are illustrated in Figures 7.26 to 7.29 ; namely, the 4-PSK 4-state trellis code; the 4-state ratc $1 / 8$ convolutional code; the 16-state 8-PSK trellis code and the 16-state rate $2 / 7$ convolutional code. Similarly, Fig:ures 7.30 to 7.33 illustrate representative cases of performance in a Rician fading chaniel without CSI; namely, the 4-PSK 4-state trellis code; the 8-PSK trellis codes; the 16-state rate 1/8 convolutional code and the 16 -state rate $2 / 7$ convolutional code.

There are several trends that are obscrvable from these results. The degree that the Caussian Approximation curves diverge from the Chernoff bound curves incroases with increasing values of $K_{f}$ as well as with the rate of the codes under consideration. More sperifically the 8-PSK trellis codes exhibit a wider divergence 


\begin{tabular}{|c|c|c|c|c|c|c|c|c|}
\hline \multirow[b]{2}{*}{ code } & \multirow[b]{2}{*}{ rate } & \multirow[b]{2}{*}{$\begin{array}{c}\text { no. } \\
\text { states }\end{array}$} & \multicolumn{3}{|c|}{$E_{b} / N_{0}[\mathrm{~dB}]$ with $\mathrm{CSI}$} & \multicolumn{3}{|c|}{$E_{b} / N_{0}[\mathrm{~dB}]$ without $\mathrm{CSI}$} \\
\hline & & & $K_{f}=10$ & $K_{f}^{\prime}=1$ & $K_{f}=0.0$ & $K_{f}=10$ & $K_{f}=1$ & $K_{l}=11.0$ \\
\hline 4-PSK & $1 / 2$ & 4 & 10.0 & - & - & 11.0 & 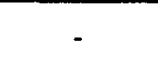 & . \\
\hline conv. & $1 / 2$ & 4 & 8.5 & 14.0 & 16.0 & 8.8 & 16.3 & 19.0 \\
\hline conv. & $1 / 2$ & 16 & 7.2 & 10.9 & 12.0 & 7.4 & 12.6 & 14.0 \\
\hline conv. & $1 / 8$ & 4 & 7.6 & 8.6 & 8.9 & 7.9 & 9.8 & 10.4 \\
\hline conv. & $1 / 8$ & 16 & 6.0 & 6.6 & 6.9 & 6.2 & 7.7 & 8.2 \\
\hline 8-PSK & $2 / 3$ & 4 & - & - & - & - & - & - \\
\hline 8.PSK & $2 / 3$ & 16 & 11.8 & - & - & 13.3 & - & - \\
\hline conv. & $2 / 7$ & 1 & 9.1 & 12.7 & 14.3 & 9.4 & 15.7 & 20.11 \\
\hline conv. & $2 / 7$ & 16 & 7.0 & 9.0 & 9.8 & 7.2 & 10.7 & 11.4 \\
\hline
\end{tabular}

Table i.1: Comparison of trellis and convolutionally coded SSMA at $P_{c}=10^{-i}$ for $K=20$ users in Rician fading, channels 
than do the 4-PSK codes. and a similar trend is observed when comparing the rate $1 / 8$ convolutional code to the rate $2 / 7$ convolutional code performance. Furthermore for the 4-PSK and 8-PSK codes, the accuracy of the Gaussian Approximation is worse for the case with no CSI. Considering all of the above codes, the degree of divergence is roughly 0.5 to $1.0 \mathrm{~dB}$ for $K_{f}=10.0$ and on the order of $1-2 \mathrm{~dB}$ for $K_{f}$ values of 1.0 and 0.0 . 


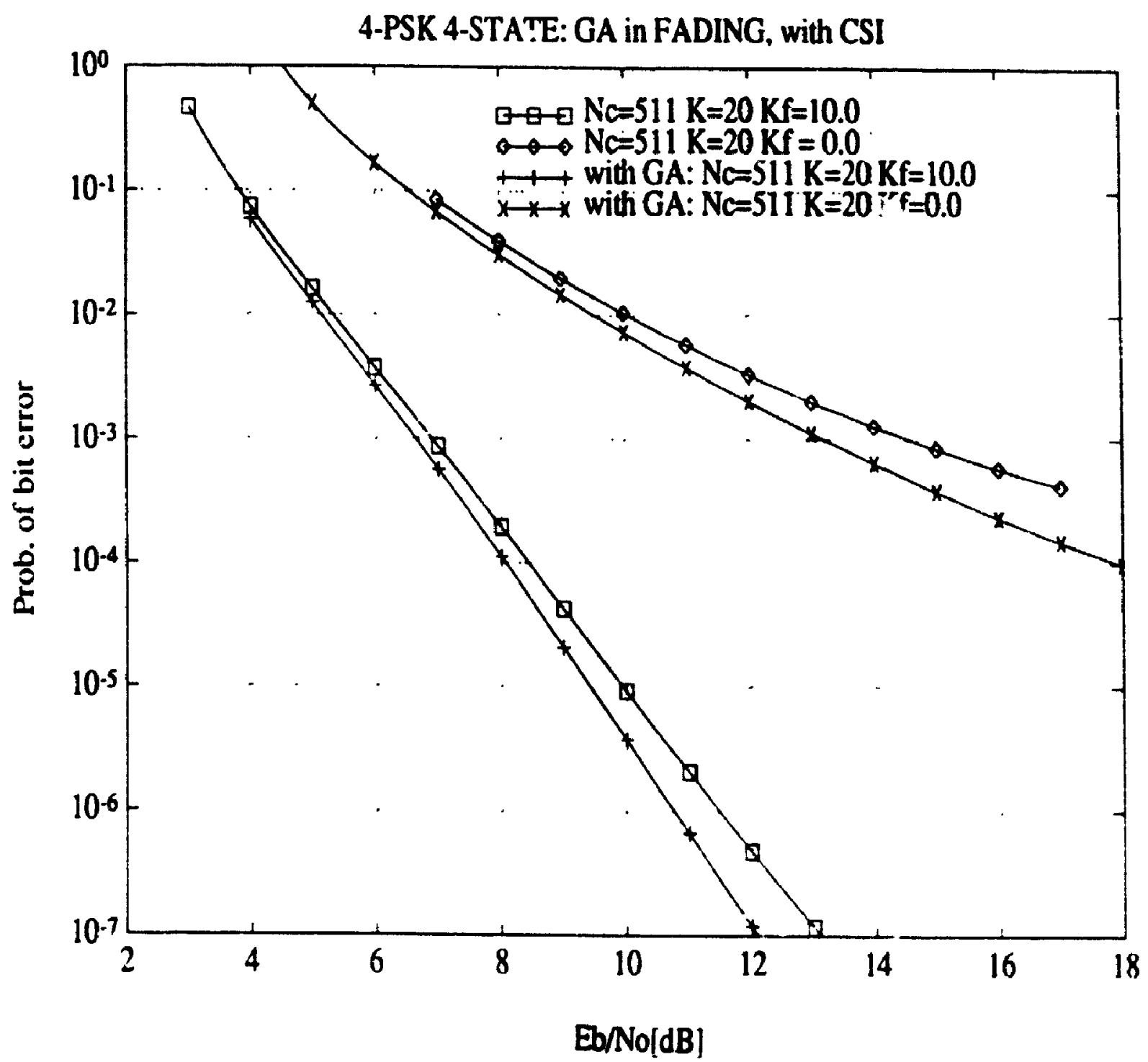

Figure 7.26: Performance of 4-PSK 4-state trellis code in Rician fading with (SI for $\mathrm{K}=20$ users employing the Gaussian Approximation $\left(N_{e}=311\right)$. 


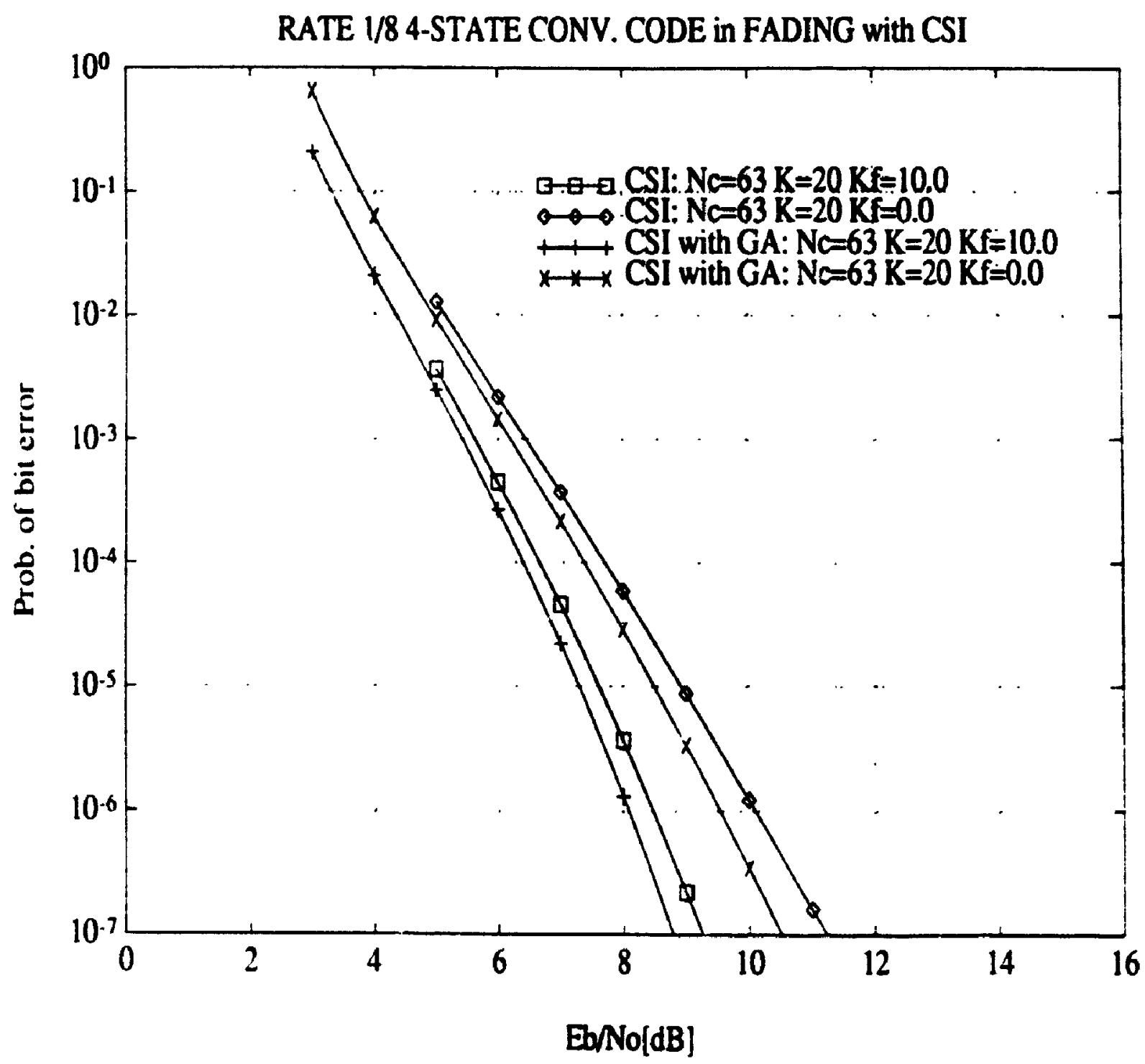

Figure 7.27 : Performance of 4-state rate $1 / 8$ convolutional code in Rician fading with ('SI for $K=20$ users employing the Gaussian Approximation $\left(N_{c}=63\right)$. 


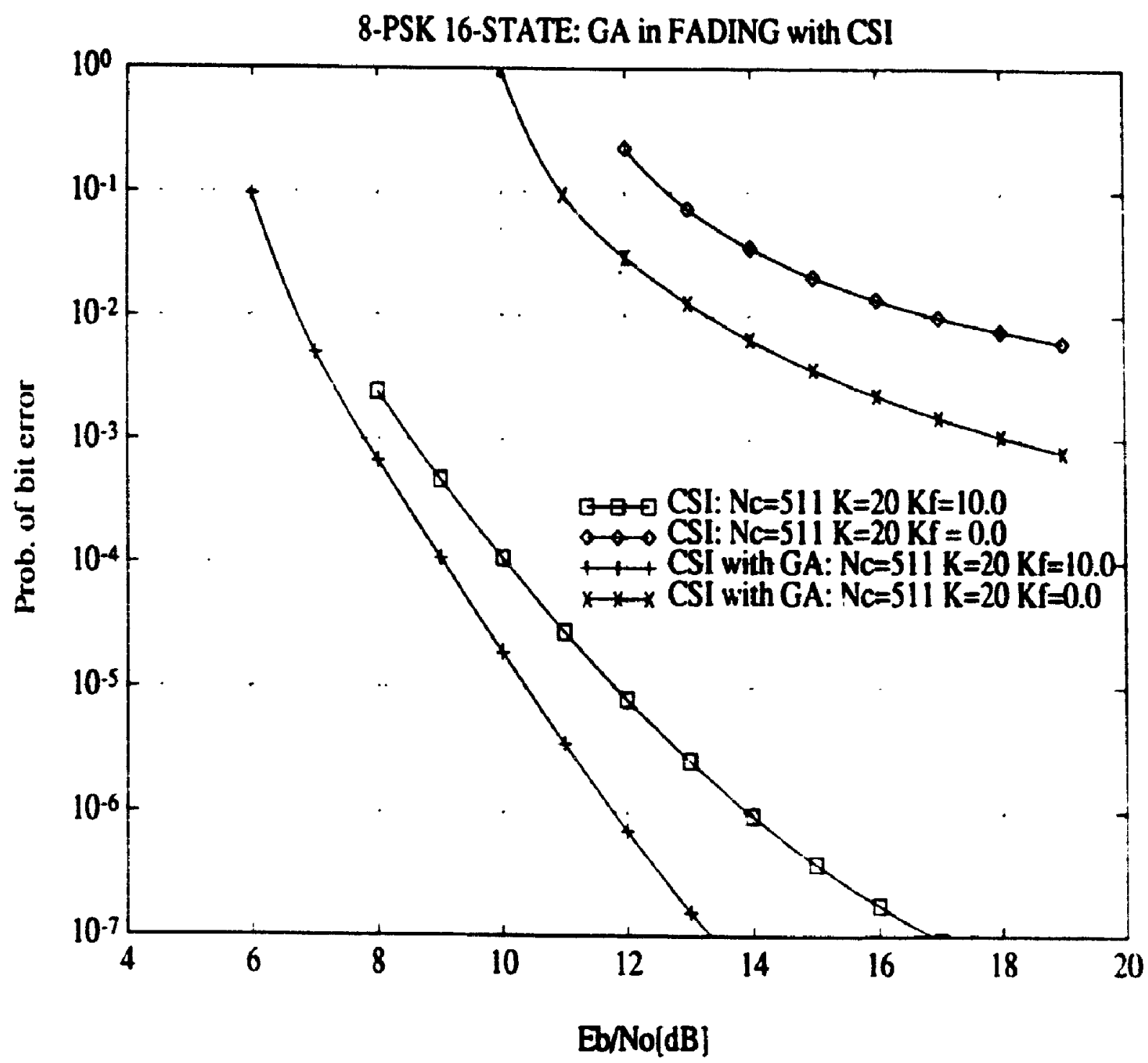

Figure 7.28: Performance of 8-PSK 16-state trellis code in Rician fading with CSI for $\mathrm{K}=20$ users employing the Gaussian Approximation $\left(N_{c}=511\right)$. 


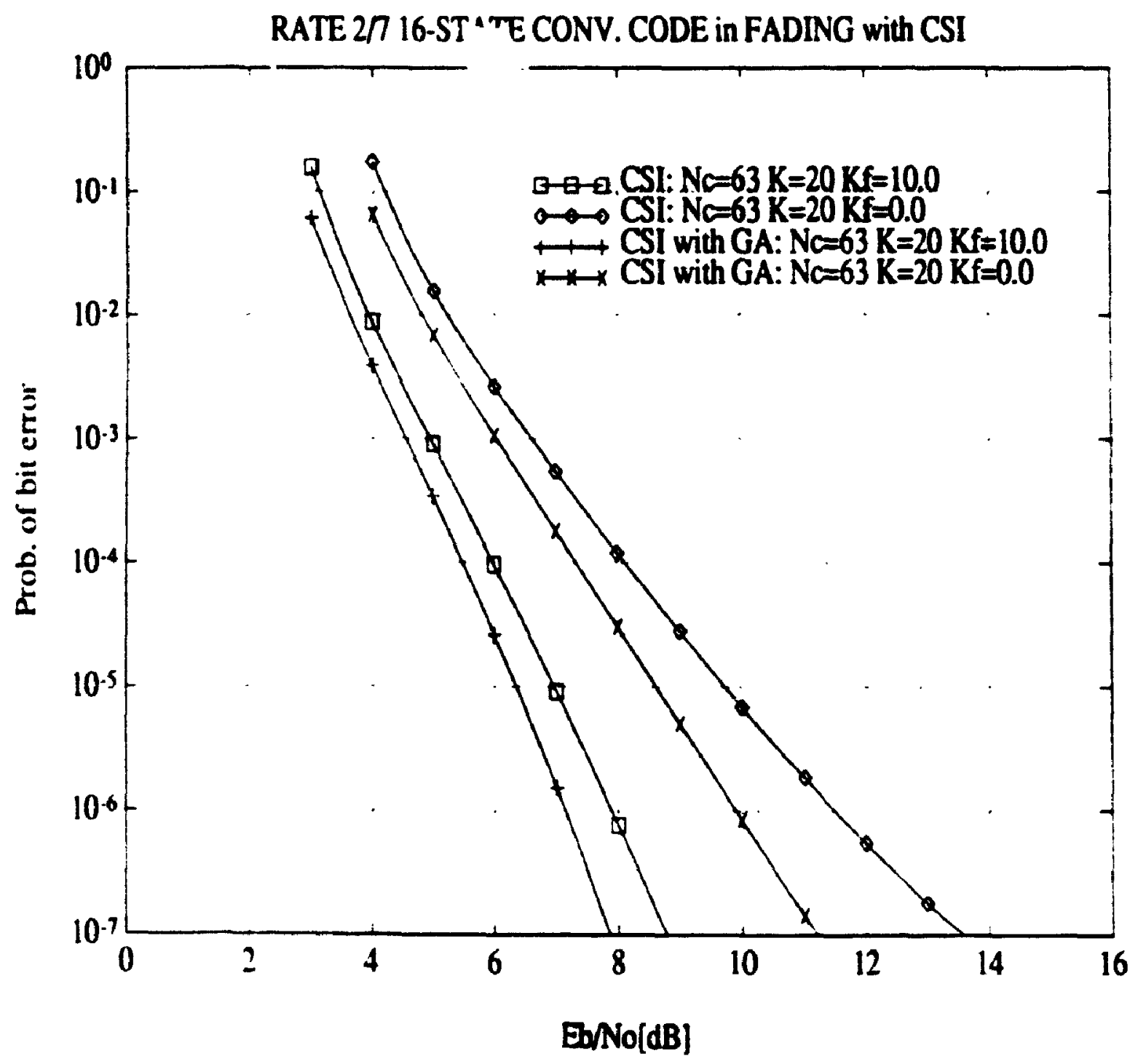

Figure $7.29:$ Performance of 16 -state rate $2 / \bar{i}$ convolutional code in Rician fading with CSl for $k=20$ users employing the Gaussian Approximation $\left(V_{c}=63\right)$. 


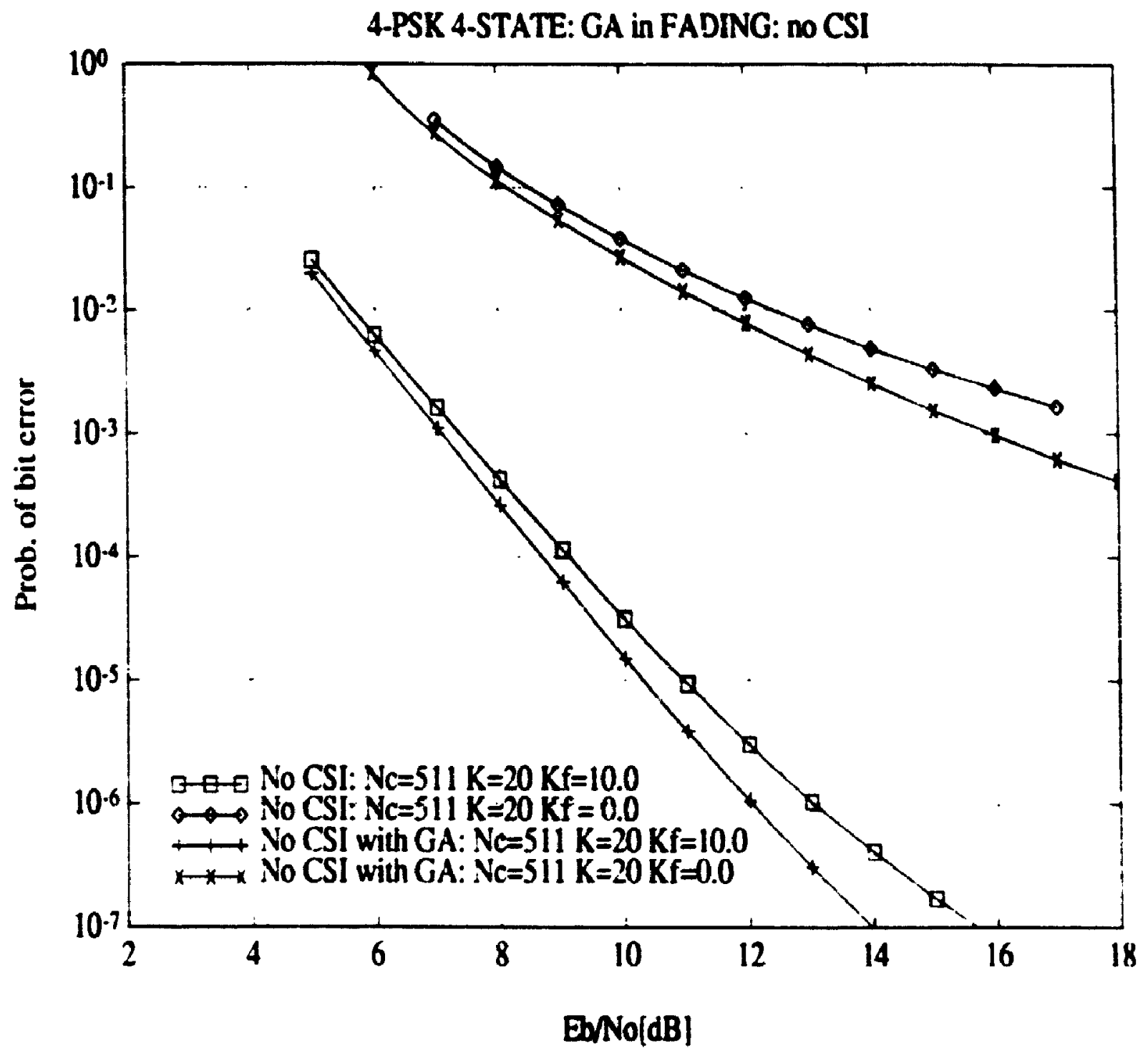

Figure 7.30: Performance of 4-PSK 4-state trellis code in llician Fading without ('SI for $\mathrm{K}=\mathbf{2 0}$ users employing the Gaussian Approximation $\left(V_{c}=511\right)$. 


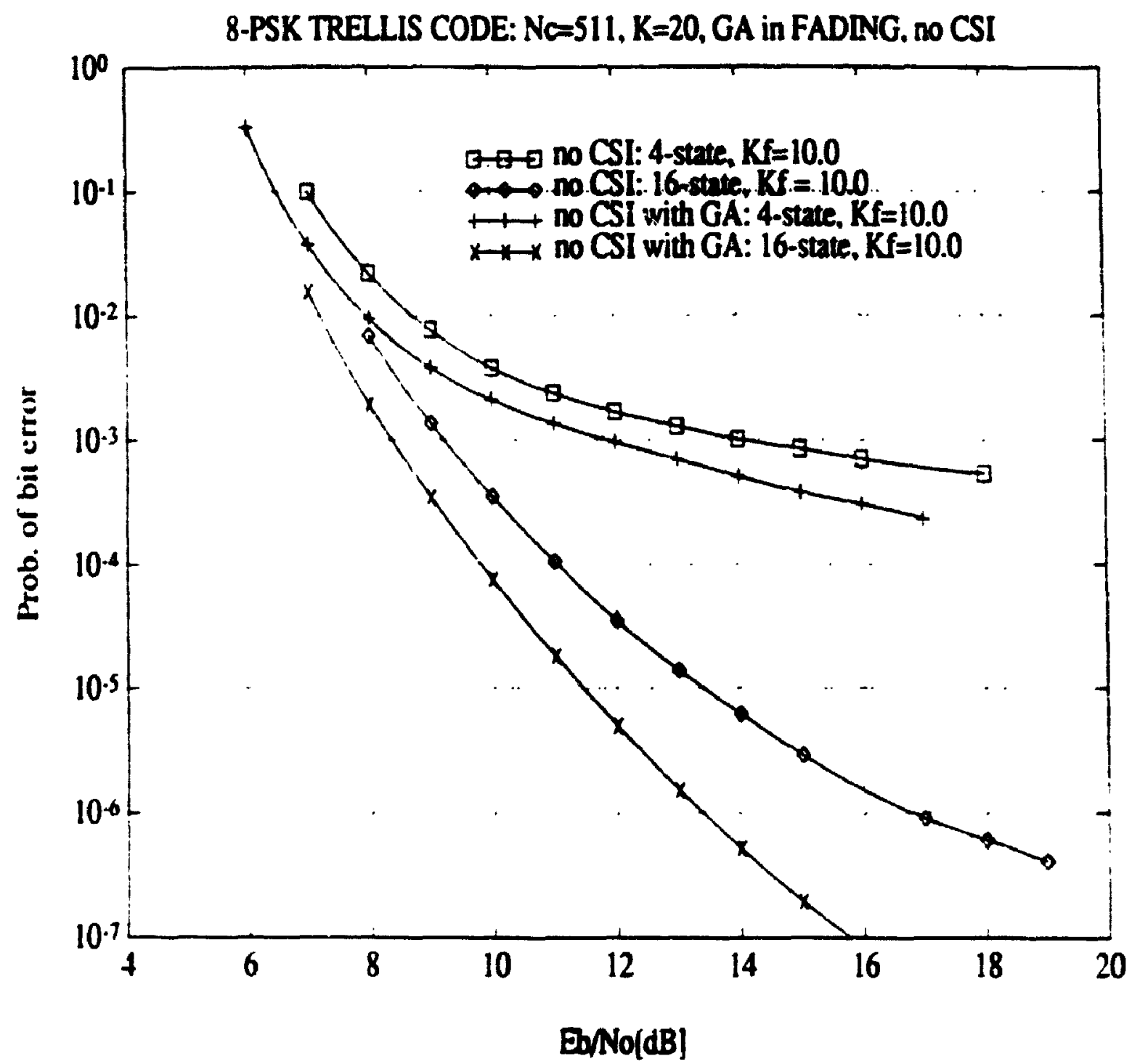

Figure 7.31: Performance of 8-PSK trellis codes in Rician fading without CSI for $K=20$ users employing the Gaussian Approximation $\left(N_{c}=511\right)$. 


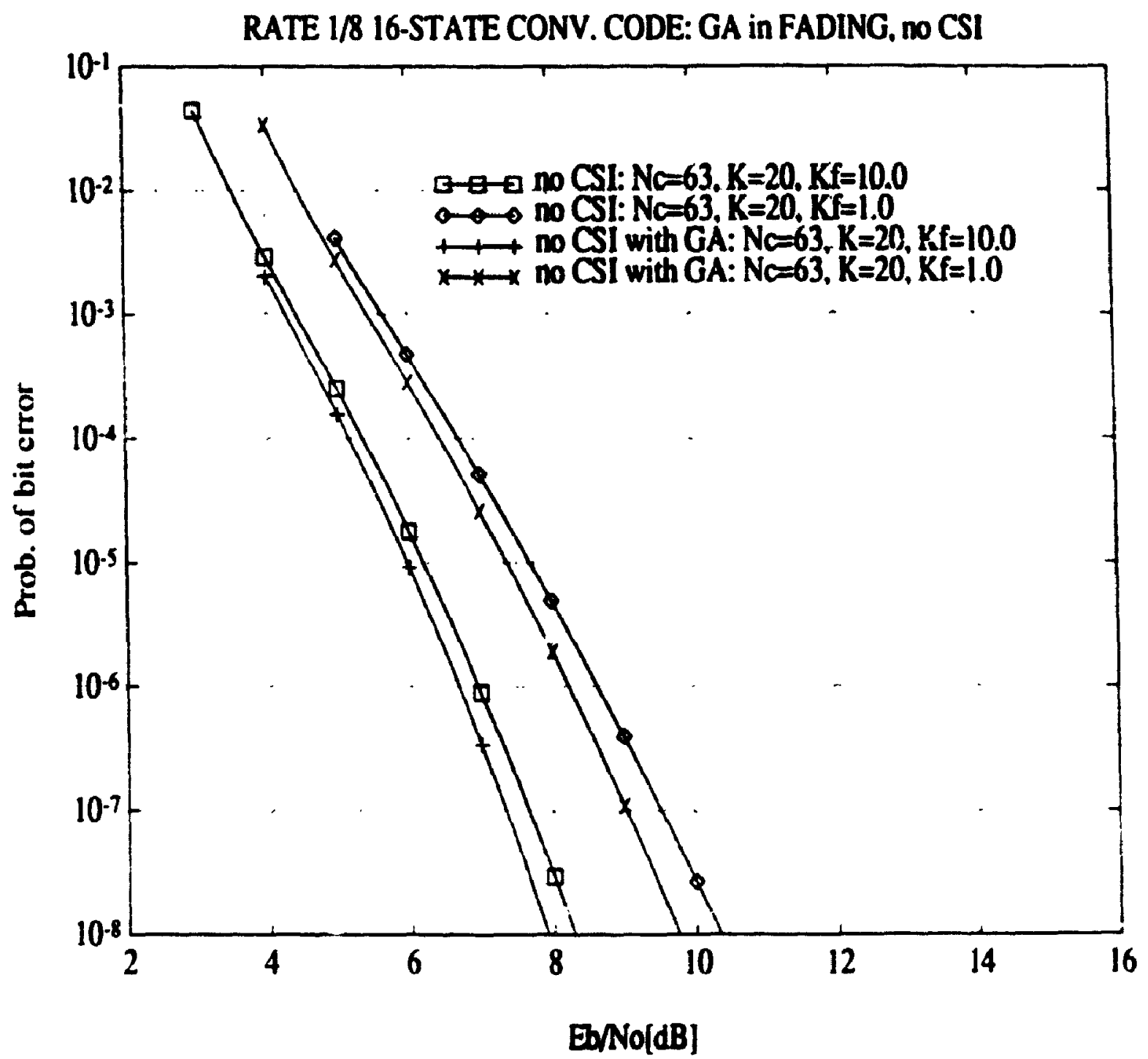

Figure 7.32: Performance of 16-state rate 1/8 convolutional code in Rician fading without CSI for $\mathrm{K}=20$ users employing the (iaussian Approximation $\left(N_{r}=6 ; 3\right)$. 


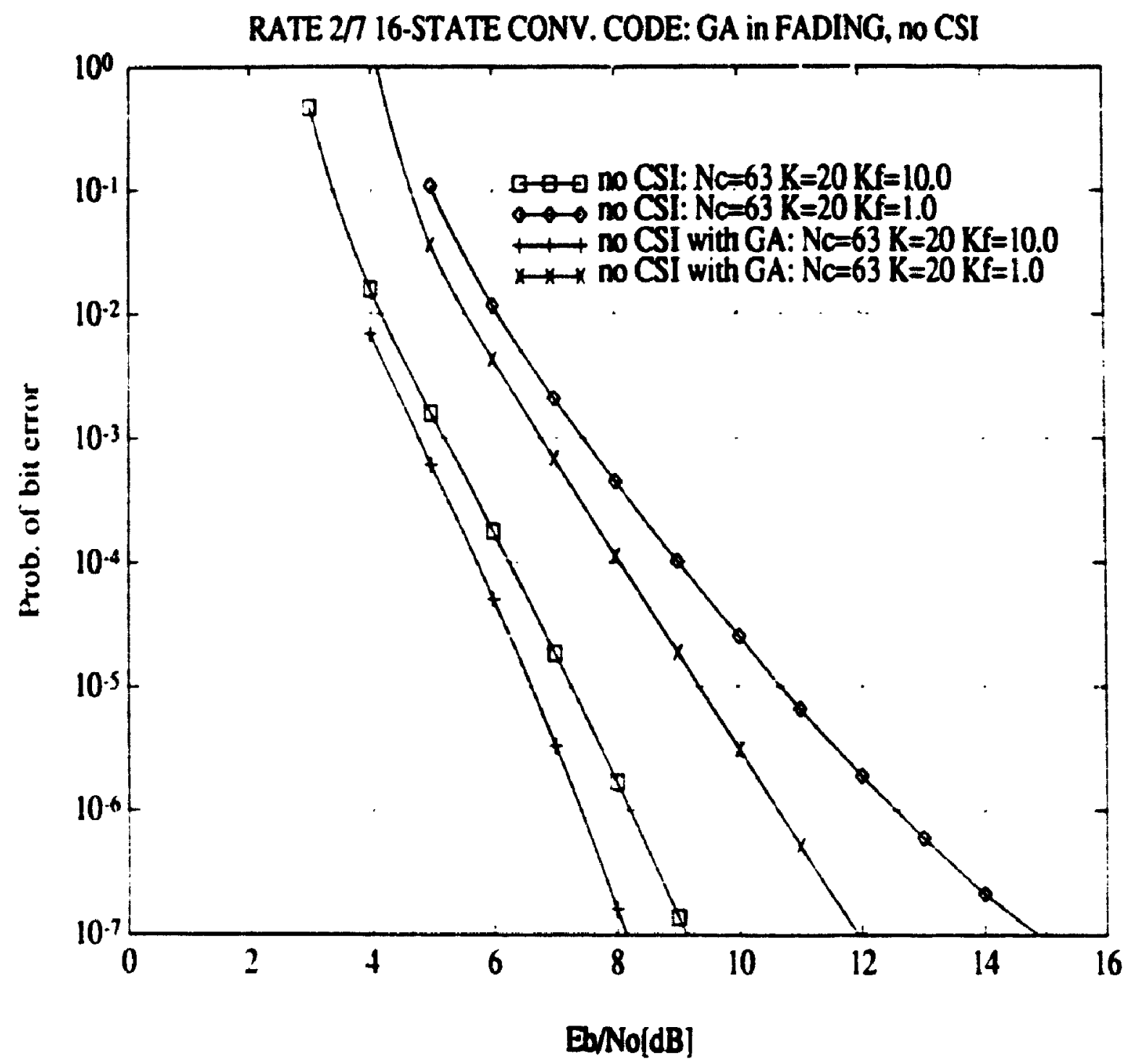

Figure 7.33: Performance of 16-state rate $2 / 7$ convolutional code in Rician fading without ( $S$ l for $K=20$ users employing the Gaussian Approximation $\left(N_{c}=63\right)$. 


\section{Chaptər 8}

\section{Conclusion}

This study has proposed and analyzed a technique through which trellis coding can be applied to an asynchronous SSMA system. The trellis codes that have been investigated for this system are the rate 1/2 4-PSK, rate 2/3 8.PSK and rate $3 / 4$ 16PSK trellis codes. Performance has been investigated for the abrve codes employing 2, 4, 8 and 16 states. The trellis codes were applied to the data information bits, with the PN spreading codes being appied to the coded symbols. (iold spreading codes up to length 511 were employed, and up to 20 users were assumerl to be present in the SSMA system. The receivers in the system were assumed to employ $I$ and $Q$ correlators followed by a Viterbi Algorithm based detertor. The performance of the above systems was upper bounded by employing a Chernoff bound in conjunction with a generalized transfer function bound algorithm which has been previously employ ad to evaluate the performance of trellis codes in an AW iN rhannel. The Chernoff bound was employed to calculate the pairwise errors between a correct symbol sequence and an error sequence from which the code performance can be upper bounded by use of the union bound (i.e. summing over all possible pairwise errors). The effects of interference due to SSMA was accounted for through the useof the method of monents. This ir volved conditioning on the inceruser interference and then averaging over the estimated probatility density function of the III (the. 
density of the IUI was accurately estimated from the moments of the IUI). The components of the IUI were in fact dependent from one symbol to the next, however for this study they were assumed to be independent. In practice this can be achieved by use of a simple interleaver of length 2 . However it was also shown that for short path lengths that this assumption of independence allows one to upper bound the true performance.

The trellis coded system for the above codes was evaluated in an AWGN channel alone and in a channel having both AWGN and Rician fading. For the AWGN noise channel it was shown that the trellis coded SSMA systems could provide gains of over 3.6dB above the uncoded SSMA systems. On the basis of a comparison of equal throughput. the 16-state rate $2 / 3$ 8-PSK code was shown to provide the best performance of the trellis codes investigated. However this may not be true for larger number of users since the 8-PSK code performance degraded more quickly with increasing numbers of users than did the 4-PSK code performance. The above results are all new.

The trellis coded SSMA system was compared to a convolutionally coded SSMA system on the basis of equal throughput and complexity (as measured by the number of states in the code). In order to achieve the same throughput for a fixed chip period the convolutional codes were assumed to employ shorter $P N$ sequences by a factor of $1 / n$ in which $k / n$ is the rate of the convolutional code (the PN sequences were assumed to span one coded symbol of the convolutional code). The performance of rate $1 / 2,1 / 8,2 / 3$ and $2 / 7$ convolutionally coded SSMA systems was evaluated under the same conditions as were the trellis coded systems. The evaluation of the performance of convolutionally coded SSMA systems has been previously analyzed in the open literature. but only using a Gaussian approximation for the interuser interference. The results of this study confirmed those results through a more exact analysis. The results indicate that the low rate convolutional codes (the rate $1 / 8$ and rate $2 / 7$ codes) provide the best performance of the codes investigated. giving gains 
of $5.0 \mathrm{~dB}$ and $5.9 \mathrm{~dB}$ respectively over the corresponding uncoded systems. Thrse low rate convolutional codes also provided roughly an additional $1-2 \mathrm{~dB}$ of improvement. in BER performance than did the comparable trellis code. This is in spite of the fact that the low rate convolutional codes had a larger contribution of IIII due to the shorter PN spreading sequences having poorer cross-correlation properties. 'The' superiority of the low rate convolutional codes over the trellis codes can be related to the larger effective minimum distance that can be achieved with the convolutional codes, whicn more than overcomes the additional iegradation due to IIII.

The performance results for the Rician fading channel exhibited similar crends in performance between the trellis and convolutionally coded SSMA systems. As for the AWGN channel, the low rate convolutional codes provided the best performance in the Rician fading channel. The trellis codes performed poorly in the Rician fadiug channel with all of the performance curves saturating at a BER above $10^{-5}$ except. for $K_{f}=10.0$.

The accuracy of employing the Gaussian approximation for the IUI was also investigated both for the trellis and the convolutional codes. It was found that this approximation was usually 1-2dB overly optimistic in its prediction of code performance as compared to the exact Chernoff bound performance.

\subsection{Future Work}

The trellis codes that were investigated were standard trellis codes that have bern optimized in an $A W G N$ channel (i.e. they maximize the Euclidean distance of the signal space). However these codes are not optimum in a SSMA channel. and as such the fact that these codes were inferior in performance to a conventional convolutionally coded system does not preclude the fact that other trellis codes could be found that provide superior performance. Thus several possible areas of fut ure work with regard to trellis coded SSMA would be to determine the optimum metric for a trellis coded system and employing this metric to search for superior trellis corles. (Mther 
parameters that could be varied in the search for superior trellis codes arc the type of spreading codes employfd, the type of constellation employed (as was pointed out in $\Lambda$ ppendix A, the combination of spreading code and constellation should be jointly optimized) and the fraction of the data symbol that a PN spreading sequence spans. Other areas of possible investigation include trellis coded SSMA systems employing higher order alphabets and multidimensional codes. 


\section{Appendix A}

\section{Evaluation of the Moments of the Interuser Interference}

In order to evaluate the performance of a SSMA system one can use the method of moments algorithm [1][22][23]. For a system which employs trellis codes, one must evaluate the probability of error $f_{c}$. path lengths which vary from one symbol to an infinite number of symbols. The method of moments algorithm requires the evaluation of the moments of the random vector $\mathrm{V}$ of equation (1.10) up to all arbitrary order $N_{m}$ at which a desired accuracy is obtained. Normalizing $V$ with respect to the symbol energy $E$, one obtains

$$
\begin{aligned}
\mathbf{E}\left[v^{m}\right] & =\mathbf{E}\left[\frac{\left(\mathbf{d}^{*}\right)^{T} \mathbf{z}+\left(\mathbf{z}^{*}\right)^{T} \mathbf{d}}{2}\right]^{m} \quad \text { for } \mathrm{m}=0,1,2 \cdots N_{m} \\
& =\mathbf{E}\left[\frac{d_{0}^{*} z_{0}+d_{0} z_{0}^{*}+d_{1}^{*} z_{1}+d_{1} z_{1}^{*}+\cdots+d_{N-1}^{*} z_{N-1}+d_{N-1} z_{N-1}^{*}}{2}\right]^{m}
\end{aligned}
$$

The numerator in the final expression of equation (A.1) can lic expresserl as a multi. 
nomial expansion giving

$$
\begin{aligned}
\mathbf{E}\left[v^{m}\right]= & \frac{1}{2^{m}} \sum \frac{m !}{i_{0} ! q_{0} ! i_{1} ! q_{1} ! \cdots i_{N-1} ! q_{N-1} !} \\
& \cdot\left(d_{0}^{*}\right)^{i_{0}}\left(d_{0}\right)^{q_{0}}\left(d_{1}^{*}\right)^{i_{0}}\left(d_{1}\right)^{q_{1}} \cdots\left(d_{N-1}^{*}\right)^{N_{-1}}\left(d_{N-1}\right)^{q_{N-1}} \\
& \cdot \mathbf{E}\left\{\left(z_{0}\right)^{i_{0}}\left(z_{0}^{*}\right)^{q_{0}}\left(z_{1}\right)^{i_{1}}\left(z_{1}^{*}\right)^{q_{1}} \cdots\left(z_{N-1}\right)^{i_{N-1}}\left(z_{N-1}^{*}\right)^{q_{N-1}}\right\} \\
= & \frac{1}{2^{m}} \sum\left[m ! \cdot \prod_{p=0}^{N-1}\left(\frac{\left(d_{p}^{*}\right)^{i_{p}}\left(d_{p}\right)^{q_{p}}}{i_{p} ! q_{p} !}\right) \mathbf{E}\left\{\prod_{p=0}^{N-1}\left(z_{p}\right)^{i_{p}}\left(z_{p}^{*}\right)^{q_{p}}\right\}\right]
\end{aligned}
$$

in which the summation is over all possible combinations of $i_{0}+q_{0}+i_{1}+q_{1}+\cdots+$ $i_{N-1}+q_{N-1}$ that sum to $m$. Furthermore, since for a given error sequence $d$. the product of the distance factors in equation (A.2) is constant, the expectation in equation (A.2) is taken over the product of the interuser interference terms

$$
\begin{aligned}
\mathcal{Z}\left(z_{p}, z_{p}^{*}\right) & =\prod_{p=0}^{v-1}\left(z_{p}\right)^{z_{p}}\left(z_{p}^{*}\right)^{q_{p}} \\
& =\left[\prod_{p=0}^{N-1}\left(G_{p}\right)^{i_{p}}\left(G_{p}^{*}\right)^{q_{p}}\right] \cdot \exp \left[j \sum_{p=0}^{N-1}\left(i_{p}-q_{p}\right) \beta\right]
\end{aligned}
$$

in which cquation (2.22) was used. Noting that the random variable 3 is uniformly distributed between $[0,2 \pi]$, it follows that the expectation of the exponential in cpuation $(A .3)$ is nonzero only when $\sum_{p=0}^{N-1}\left(i_{p}-q_{p}\right)=0$. in which case the exponential takes on a value of unity. Thus equation (A.2) can be expressed as

$$
\mathbf{E}\left[r^{m}\right]=\sum\left[\frac{m !}{2^{m}} \prod_{p=0}^{v-1}\left(\frac{\left(d_{p}^{*}\right)^{z_{p}}\left(d_{p}\right)^{q_{p}}}{i_{p} ! q_{p} !}\right) \mathbf{E}\left\{\prod_{p=0}^{N-1}\left(G_{p}\right)^{i_{p}}\left(G_{p}^{*}\right)^{q_{p}}\right\}\right]
$$

in which the summation is over all combinations of $i_{0}+q_{0}+i_{1}+q_{1}+\cdots+i_{N-1}+q_{N-1}$ that sum to $m$ and simultaneously satisfy the condition $\sum_{p=0}^{N-1}\left(i_{p}-q_{p}\right)=0$.

The expectation is taken over the product of the random variables $G_{p}$ and $G_{p}^{*}$ which can be defined as

$$
\mathcal{G}\left(G_{p} . G_{p}^{*}\right)=\prod_{p=0}^{N-1}\left\{\left(G_{p}\right)^{\prime p}\left(G_{p}^{*}\right)^{q_{p}}\right\}
$$


Define the real and imaginary parts of the random variable $G_{p}$ as $E_{p}$ and $F_{p}$, the definitions of which can be found in equations (D.9) and (D.10) of Appendix D. The resulting expression for $\mathcal{G}\left(G_{p}, G_{p}^{*}\right)$ becomes

$$
\begin{aligned}
\mathcal{G}\left(G_{p}, G_{p}^{*}\right)= & \prod_{p=0}^{N-1}\left\{\left[\frac{1}{T} \sum_{\substack{k=1 \\
k \neq i}}^{K}\left[E_{p}^{k}+j F_{p}^{k}\right]\right]^{\top p}\right. \\
& \left.\cdot\left[\frac{1}{T} \sum_{\substack{k=1 \\
k \neq i}}^{K}\left[E_{p}^{k}-j F_{p}^{k}\right]\right]^{q p}\right\}
\end{aligned}
$$

Due to the assumed randomness of transmissions from one user to the next, the interuser interference random variables are independent (between users), and as such one may calculate the interference random variable $v^{k}$ due to the $k^{\text {th }}$ user independently of the other users. Once $v^{k}$ is obtained for each of the users, the total interuser interference can be obtainod using the cumulants algorithm[1] based on the fact that

$$
v=\sum_{\substack{k=1 \\ k \neq i}}^{K} v^{k}
$$

Considering equation (A.6) for any interferer $k$, and neglecting the user superseript without any loss of generality, one obtains

$$
\begin{aligned}
& \mathcal{G}\left(G_{p}, G_{p}^{*}\right)=\prod_{p=0}^{. v-1}\left\{\frac{1}{T_{p p}^{p_{p}+q_{p}}}\left[E_{p}+j F_{p}\right]^{{ }_{p} p}\right. \\
&\left.\cdot\left[E_{p}-j F_{p}\right]^{q_{p}}\right\}
\end{aligned}
$$

Expanding equation (A.8) gives

$$
\begin{aligned}
\mathcal{G}\left(G_{p}, G_{p}^{*}\right)= & \prod_{p=0}^{N-1}\left\{\sum_{a_{p}=0}^{i_{p}}\left(\begin{array}{l}
i_{p} \\
a_{p}
\end{array}\right)(j)^{t_{p}-a_{p}} E_{p}^{a_{p}} F_{p}^{i_{p}-a_{p}}\right. \\
& \left.\cdot \sum_{b_{p}=0}^{q_{p}}\left(\begin{array}{l}
q_{p} \\
b_{p}
\end{array}\right)(-J)^{z_{p}-b_{p}} E_{p}^{b_{p}} F_{p}^{q_{p}-b_{p}}\right\}
\end{aligned}
$$




$$
\begin{aligned}
& =\prod_{p=0}^{N-1}\left\{\sum_{c_{p}=0}^{i_{p}} \sum_{b_{p}=0}^{a_{p}}\left(\begin{array}{l}
i_{p} \\
a_{p}
\end{array}\right)\left(\begin{array}{l}
q_{p} \\
b_{p}
\end{array}\right)(j)^{i_{p}+a_{p}-a_{p}-b_{p}}\right. \\
& \text { - } \left.E_{p}^{a_{p}+b_{p}} F_{p}^{i_{p}+a_{p}-a_{p}-b_{p}}\right\} \\
& =\sum_{a_{0}=0}^{2_{0}} \sum_{b_{0}=0}^{q_{0}} \sum_{a_{1}=0}^{i_{1}} \sum_{b_{1}=0}^{q_{1}} \ldots \sum_{a_{N-1}=0}^{i_{N-1}} \sum_{b_{N-1}=0}^{q_{N-1}} \\
& \cdot\left(\begin{array}{l}
i_{0} \\
a_{0}
\end{array}\right)\left(\begin{array}{l}
q_{0} \\
b_{0}
\end{array}\right)\left(\begin{array}{l}
i_{1} \\
a_{1}
\end{array}\right)\left(\begin{array}{l}
q_{1} \\
b_{1}
\end{array}\right) \cdots\left(\begin{array}{l}
i_{N-1} \\
a_{N-1}
\end{array}\right)\left(\begin{array}{l}
q_{N-1} \\
b_{N-1}
\end{array}\right)
\end{aligned}
$$

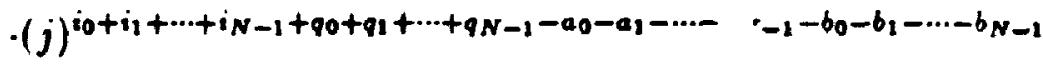

$$
\begin{aligned}
& \cdot \prod_{p=0}^{N-1}\left\{E_{p}^{a p+b_{p}} F_{p}^{a_{p}+a_{p}-a_{p}-b_{p}}\right\}
\end{aligned}
$$

Taking the expectation of equation (A.9) results in

$$
\begin{aligned}
& \mathbf{E}\left[\mathcal{G}\left(G_{p}, G_{p}^{*}\right)\right]=\sum_{a_{0}=0}^{10} \sum_{b_{0}=0}^{90} \sum_{a_{1}=0}^{11} \sum_{b_{1}=0}^{q_{1}} \ldots \sum_{a_{N-1}=c}^{i_{N}-1} \sum_{b_{N-1}=0}^{i_{N}-1} \\
& \cdot\left(\begin{array}{l}
i_{0} \\
a_{0}
\end{array}\right)\left(\begin{array}{l}
q_{0} \\
b_{0}
\end{array}\right)\left(\begin{array}{l}
i_{1} \\
a_{1}
\end{array}\right)\left(\begin{array}{l}
q_{1} \\
b_{1}
\end{array}\right) \cdots\left(\begin{array}{l}
i_{N-1} \\
a_{N-1}
\end{array}\right)\left(\begin{array}{l}
q_{N-1} \\
b_{N-1}
\end{array}\right) \\
& \cdot(-j)^{20+1_{1}+\cdots+1_{N-1}+q_{0}+q_{1}+\cdots+q_{N-1}-a_{0}-a_{1}-\cdots-a_{N-1}-b_{0}-b_{1}-\cdots-b_{N-1}} \\
& \cdot \mathbf{E}\left\{\prod_{p=0}^{N-1}\left(E_{p}^{a_{p}+b_{p}} F_{p}^{t_{p}+a_{p}-a_{p}-b_{p}}\right)\right\}
\end{aligned}
$$

Thus the problem reduces to determining the expectation of the expression on the righthand side of equation (A.10).The expectation of the function of the random variables $E_{p}$ and $F_{p}$ can be evaluated using the definitions of equations (D.9) and (D.10). Defining $\mathcal{F}\left(E_{p} . F_{p}\right)$ as the final product in equation (A.10) gives

$$
\begin{aligned}
\mathbf{E}\left\{\mathcal{F}\left(E_{p}, F_{p}\right)\right\}= & \mathbf{E}\left\{\prod_{p=0}^{N-1}\left[E_{p}^{a_{p}+b_{p}} F_{p}^{i p+a_{p}-a_{p}-b_{p}}\right]\right\} \\
= & \mathbf{E}\left\{\prod _ { p = 0 } ^ { N - 1 } \left[\left(x_{l_{p-1}}^{k} R_{k, 3}\left(\tau^{k}\right)+x_{l_{p}}^{k} \hat{R}_{k, 2}\left(\tau^{k}\right)\right)^{a_{p}+b_{p}}\right.\right.
\end{aligned}
$$




$$
\left.\left.\cdot\left(x_{Q_{p-1}}^{k} R_{k, i}\left(\tau^{k}\right)+x_{Q_{p}}^{k} \dot{R}_{k, 1}\left(\tau^{k}\right)\right)^{t_{p}+q_{p}-a_{p}-b_{p}}\right]\right\}
$$

Defining $u_{p}$ and $v_{p}$ as

$$
\begin{aligned}
& u_{p}=a_{p}+b_{p} \\
& v_{p}=i_{p}+q_{p}-a_{p}-b_{p}
\end{aligned}
$$

equation (A.11) can be expressed as

$$
\begin{aligned}
& \mathbf{E}\left\{\mathcal{F}\left(E_{p}, F_{p}\right\}\right\}=\mathbf{E}\left\{\prod _ { p = 0 } ^ { N - 1 } \left[\sum_{e_{p}=0}^{u_{p}}\left(\begin{array}{l}
u_{p} \\
e_{p}
\end{array}\right)\left(x_{l_{p-1}}^{k}\right)^{e_{p}}\left(x_{I_{p}}^{k}\right)^{u_{p}-e_{p}}\right.\right. \\
& \cdot\left(R_{k, 2}\left(\tau^{k}\right)\right)^{e_{p}}\left(\hat{R}_{k, i}\left(\tau^{k}\right)\right)^{u_{p}-e_{p}} \\
& \text { - } \left.\left.\sum_{f_{p}=0}^{v_{p}}\left(\begin{array}{l}
v_{p} \\
f_{p}
\end{array}\right)\left(x_{Q_{p-1}}^{k}\right)^{f_{p}}\left(x_{Q_{p}}^{k}\right)^{v_{p}-f_{p}}\left(R_{k, 1}\left(\tau^{k}\right)\right)^{f_{p}}\left(\hat{R}_{k, 1}\left(r^{k}\right)\right)^{p_{p}-f_{p}}\right]\right\} \\
& =\mathbf{E}\left\{\prod _ { p = 0 } ^ { N - 1 } \left[\sum_{e_{p}=0}^{u_{p}} \sum_{f_{p}=0}^{u_{p}}\left(\begin{array}{l}
u_{p} \\
e_{p}
\end{array}\right)\left(\begin{array}{l}
v_{p} \\
f_{p}
\end{array}\right)\right.\right. \\
& \cdot\left(x_{I_{p-1}}^{k}\right)^{e_{p}}\left(x_{Q_{p-1}}^{k}\right)^{f_{p}}\left(x_{I_{p}}^{k}\right)^{u_{p}-e_{p}}\left(x_{Q_{p}}^{k}\right)^{1_{p}-f_{p}} \\
& \left.\left.\cdot\left(R_{k, i}\left(\tau^{k}\right)\right)^{e_{p}+f_{p}}\left(\hat{R}_{k, i}\left(\tau^{k}\right)\right)^{u_{p}+v_{p}-e_{p}-f_{p}}\right]\right\} \\
& =\sum_{e_{p}=0}^{u_{0}} \sum_{f_{p}=0}^{u_{0}} \sum_{e_{p}=0}^{u_{1}} \sum_{f_{p}=0}^{u_{1}} \cdots \sum_{e_{p}=0}^{u_{N}-1} \sum_{f_{p}=0}^{v_{N-1}} \\
& \left(\begin{array}{l}
u_{p} \\
e_{0}
\end{array}\right)\left(\begin{array}{c}
v_{p} \\
f_{0}
\end{array}\right)\left(\begin{array}{c}
u_{p} \\
e_{1}
\end{array}\right)\left(\begin{array}{c}
v_{p} \\
f_{1}
\end{array}\right) \cdots\left(\begin{array}{c}
u_{p} \\
e_{N-1}
\end{array}\right)\left(\begin{array}{c}
v_{p} \\
f_{N-1}
\end{array}\right) \\
& \mathbf{E}\left\{\left(x_{I_{-1}}^{k}\right)^{\epsilon_{0}}\left(x_{Q_{-1}}^{k}\right)^{f_{0}} \prod_{p=0}^{N-2}\left[\left(x_{I_{p}}^{k}\right)^{u_{p}-r_{p}+e_{p+1}}\left(x_{Q_{p}}^{k}\right)^{\nu_{p}-f_{p}+f_{p+1}}\right]\right. \\
& \left.\cdot\left(x_{I_{N-1}}^{k}\right)^{u_{N-1}-e_{N-1}}\left(x_{Q_{N-1}}^{k}\right)^{u_{N-1}-e_{N-1}}\right\} \\
& \cdot \mathbf{E}\left\{\prod_{p=0}^{N-1}\left[\left(R_{k, 2}\left(\tau^{k}\right)\right)^{e_{p}+f_{p}}\left(\hat{R}_{k, 1}\left(\tau^{k}\right)\right)^{u_{p}+\nu_{p}-t_{p}-f_{p}}\right]\right\}
\end{aligned}
$$

In the derivation of equation (A.14) the expectation was split into two prodisets, 
based on the fact that the in-phase and quadrature components of the transmitted symbols of the interferers (i.e. $x_{I_{p}}^{k}$ and $x_{Q_{p}}^{k}$ ) are independent of the continuoustime partial cross-correlation functions $R_{k, 8}\left(\tau^{k}\right)$ and $\hat{R}_{k, i}\left(\tau^{k}\right)$. Define the expectation of the first product as $\mathcal{X}\left(x_{l_{p}}^{k}, x_{Q_{p}}^{k}\right)$ and the expectation of the second product as $\mathcal{R}(R, \grave{R})$. Simplifying $\mathcal{R}(R, \hat{R})$ gives

$$
\mathrm{E}\left\{\prod_{p=0}^{N-1}\left[\left(R_{k, 1}\left(\tau^{k}\right)\right)^{e_{p}+f_{p}}\left(\hat{R}_{k, 1}\left(\tau^{k}\right)\right)^{u_{p}+u_{p}-e_{p}-f_{p}}\right]\right\}=\mathrm{E}\left\{\left(R_{k, 1}\left(\tau^{k}\right)\right)^{r^{\prime}}\left(\hat{R}_{k, 1}\left(\tau^{k}\right)\right)^{g^{\prime}}\right\}
$$

in which

$$
\begin{aligned}
r^{\prime} & =\sum_{p=0}^{. v-1}\left[e_{p}+f_{p}\right] \\
s^{\prime} & =\sum_{p=0}^{N-1}\left[u_{p}+v_{p}-e_{p}-f_{p}\right]
\end{aligned}
$$

For a given set of spreading sequences $\left\{a^{(k)}(t)\right\}$ equation $(A .15)$ can be evaluated using numerical techniques. Calculation of $\mathcal{X}\left(x_{I_{p}}^{k}, x_{Q_{p}}^{k}\right)$ depends on the constellation being employed to transmit the data. Expressions for $\boldsymbol{K}\left(x_{l_{p}}^{k}, x_{Q_{p}}^{k}\right)$ are derived in Ippendix E for various commonly employed constellations.

All of the expressions required to calculate the moments of equation (A.1) have now been defined. Recapitulating, the moments of equation $(A .1)$ can be calculated numerically in the following steps. Initially the functions $\mathcal{R}(R, \dot{R})$ and $\boldsymbol{l}\left(x_{l_{p}}^{k}, x_{Q_{p}}^{k}\right)$ can be calculated as defined in equations $(\mathcal{A} .14)$ and $(\lambda .15)$. These results can subsequently be utililized to evaluate $\mathcal{F}\left(E_{p}, F_{p}\right)$ of equation $(A .11)$. Moreover $\mathcal{F}\left(E_{p}, F_{p}\right)$ can be used to determine $\mathcal{G}\left(G_{p}, G_{p}^{*}\right)$ as defined in equation (A.10). Lastly the function $\Xi\left(z_{p} z_{p}^{*}\right)$ is used to evaluate the moments of equation $(A .1)$.

From the above analysis of the moments it can be seen that the interuser interfer'nce is a function of both the cross-correlation properties of the set of PN sequences employed for spreading the transmitted signals (i.e. through the factor $\mathcal{R}(R . \hat{R})$ ). and the signal constellation employed by the trellis code (i.e. the factor $\mathfrak{l}\left(x_{J_{p}}^{k}, x_{Q_{p}}^{k}\right)$ ). 
Thus in order to optimize the performance of the trellis code one must optimize $\mathcal{R}(R, \hat{R})$ in conjunction with $\mathfrak{l}\left(x_{l_{p}}^{k}, x_{Q_{p}}^{k}\right)$.

\section{A.1 Evaluation of Moments Under the Indepen- dence Assumption}

If it is assumed that the interuser interference random variable $z_{p}$ is independent from one symbol to the next. then the evaluation of equation $(A .1)$ is simplified considerably since $V$ is equal to one. Dropping the superscript $p$ without loss of generality and evaluating only even moments (the odd moments are zero since the density function of $Z$ is even), gives

$$
\begin{aligned}
\mathbf{E}\left[v^{2 m}\right] & =\frac{1}{4^{m}} \mathbf{E}\left[\sum_{i=0}^{2 m}\left(\begin{array}{c}
2 m \\
i
\end{array}\right)\left(d^{*} \cdot z\right)^{i}\left(d \cdot z^{-}\right)^{2 m-1}\right] \\
& =\frac{1}{4^{m}} \sum_{i=0}^{2 m}\left(\begin{array}{c}
2 m \\
i
\end{array}\right)\left(d^{*}\right)^{*}(d)^{2 m-i} \mathbf{E}\left[(z)^{2}\left(z^{*}\right)^{2 m-1}\right]
\end{aligned}
$$

Substituting the expression for $z$ of equation (2.21) into equation (A.18) gives

$$
\mathbf{E}\left[1^{2 m}\right]=\frac{1}{4^{m}} \sum_{i=0}^{2 m}\left(\begin{array}{c}
2 m \\
1
\end{array}\right) \mathbf{E}\left[(G)^{3}\left(G^{\prime *}\right)^{2 m-1}\right] \mathbf{E}[\exp (j(2 m-2 i) / \beta)]
$$

Noting that the second expectation in equation $(A .19)$ is nonzero only when $i=m$ enables one to simplify the expression to

$$
\mathbf{E}\left[W^{2 m}\right]=\frac{1}{4^{m}}\left(\begin{array}{c}
2 m \\
m
\end{array}\right)|d|^{2 m} \mathbf{E}\left[\left|C_{i}\right|^{2 m}\right]
$$




\section{Appendix B}

\section{Moments of a Normalized Rician Random Variable}

The corventional Rician density function is expressed as [110]

$$
p_{R}\left(r_{f}\right)=\frac{r_{f}}{\sigma^{2}} \exp \left[-\frac{\left(r_{f}^{2}+s^{2}\right)}{2 \sigma^{2}}\right] I_{0}\left(\frac{r_{f} s}{\sigma^{2}}\right)
$$

in which $s^{2}$ is the power in the direct line of sight path, $2 \sigma^{2}$ is the power in the diffuse component and $I_{0}(\cdot)$ is the zeroth order modified Bessel function of the first kind. Define the normalized random amplitude as $\rho=a \cdot r_{f}$ in which[119]

$$
a^{2}=\frac{1}{s^{2}+2 \sigma^{2}}
$$

Therefore. it follows from probability theory that

$$
\begin{aligned}
P_{\rho}(\rho) & =\frac{1}{a} p_{R}\left(\frac{\rho}{a}\right) \\
& =\sqrt{s^{2}+2 \sigma^{2}} \cdot p_{R}\left(\rho \sqrt{\left.s^{2}+2 \sigma^{2}\right)}\right. \\
& =\rho \frac{\left(s^{2}+2 \sigma^{2}\right)}{\sigma^{2}} \exp \left[\frac{-\rho^{2}\left(s^{2}+2 \sigma^{2}\right)-s^{2}}{2 \sigma^{2}}\right] \cdot I_{0}\left(2 \rho \sqrt{\frac{s^{2}\left(s^{2}+2 \sigma^{2}\right)}{4 \sigma^{2}}}\right)
\end{aligned}
$$

Dedining $K_{f}=\kappa^{2} / 2 \sigma^{2}$. and substituting this value into equation (B.3) gives the 
amplitude normalized form of the Rician density function

$$
p_{\rho}(\rho)=2 \rho\left(K_{f}+1\right) \cdot \exp \left[-\rho^{2}\left(K_{f}+1\right)-K_{f}\right] \cdot I_{0}\left(2 \rho \sqrt{K_{f}\left(K_{f}+1\right)}\right)
$$

In the calculation of the interuser interference random variable $:$ in a Rician fading channel it is necessary to calculate the even moments of $\rho$ of equation (B.1). Since

$$
E\left[\rho^{2 m}\right]=\int_{0}^{\infty} \rho^{2 m} \cdot p_{p}(\rho) d \rho
$$

it follows from equation (B.4) that

$$
E\left[\rho^{2 m}\right]=\int_{0}^{\infty} \rho^{2 m} \cdot 2 \rho\left(K_{f}+1\right) \cdot \exp \left[-\rho^{2}\left(K_{f}+1\right)-K_{f}\right] \cdot I_{0}\left(2 \rho \sqrt{K_{f}\left(K_{f}+1\right)}\right) d \rho
$$

Furthermore, using the fact that[117]

$$
I_{0}(x)=\sum_{n=0}^{\infty} \frac{1}{(n !)^{2}} \cdot\left(\frac{x}{2}\right)^{2}
$$

equation (B.6) becomes

$$
\begin{aligned}
\mathbf{E}\left[\rho^{2 m}\right]= & 2\left(1+K_{f}\right) \exp \left(-K_{f}\right) \cdot \\
& \left.\int_{0}^{\infty} \rho^{2 m+1} \sum_{n=0}^{\infty}\left\{\frac{1}{(n !)^{2}} \cdot \rho^{2 n} ! K_{f}\left(K_{f}+1\right)\right]^{n}\right\}\left(\operatorname{xpp}\left[-\rho^{2}\left(1+K_{f}\right) \mid d \rho\right)\right. \\
= & 2\left(1+K_{f}\right) \exp \left(-K_{f}\right) \cdot \\
& \sum_{n=0}^{\infty}\left\{\left[K_{f}\left(K_{f}+1\right)\right]^{n} \int_{0}^{\infty} \frac{\rho^{2 m+2 n+1}}{(n !)^{2}} \cdot \exp \left[-\rho^{2}\left(1+K_{f}\right)\right] d \rho\right\} \quad(B . X)
\end{aligned}
$$

Using the fact that [117]

$$
\int_{0}^{\infty} x^{2 n+1} \exp \left(-\phi x^{2}\right) d x=\frac{n !}{2 \phi^{n+1}}
$$

equation (B.8) can be simplified to

$$
\mathbf{E}\left[\rho^{2 m}\right]=2\left(1+K_{f}\right) \exp \left(-K_{f}\right) \sum_{n=0}^{\infty} \frac{\left[K_{f}\left(1+K_{f}\right]^{n}\right.}{(n !)^{2}} \cdot \frac{(m+n) !}{2\left(1+K_{f}\right)^{m+n+1}}
$$


Simplifying further gives

$$
\mathbf{E}\left[\rho^{2 m}\right]=\frac{\exp \left(-K_{f}\right)}{\left(1+K_{j}\right)^{m}} \cdot m ! \cdot \sum_{n=0}^{\infty}\left(\begin{array}{c}
m+n \\
n
\end{array}\right) \frac{K_{f}^{n}}{n !}
$$

The expression in equation (B.11) is an iffinite series which is not of practical use if one wishes to evaluate the moments of the Rician density function accurately. However equation (B.11) can be converted to a finite series that can be evaluated, through use of the following combinatorial identity[117]

$$
\left(\begin{array}{c}
m+n \\
p
\end{array}\right)=\left(\begin{array}{c}
m \\
0
\end{array}\right)\left(\begin{array}{l}
n \\
p
\end{array}\right)+\left(\begin{array}{c}
m \\
1
\end{array}\right)\left(\begin{array}{c}
n \\
p-1
\end{array}\right)+\cdots\left(\begin{array}{l}
m \\
p
\end{array}\right)\left(\begin{array}{l}
n \\
0
\end{array}\right)
$$

which allows one to express the combinatorial in equation (B.11) as

$$
\begin{aligned}
\left(\begin{array}{c}
m+n \\
n
\end{array}\right) & =\left(\begin{array}{c}
m \\
0
\end{array}\right)\left(\begin{array}{l}
n \\
n
\end{array}\right)+\left(\begin{array}{c}
m \\
1
\end{array}\right)\left(\begin{array}{c}
n \\
n-1
\end{array}\right)+\cdots\left(\begin{array}{l}
m \\
n
\end{array}\right)\left(\begin{array}{l}
n \\
0
\end{array}\right) \\
& =\left(\begin{array}{c}
m \\
0
\end{array}\right)\left(\begin{array}{l}
n \\
n
\end{array}\right)+\left(\begin{array}{c}
m \\
1
\end{array}\right)\left(\begin{array}{c}
n \\
n-1
\end{array}\right)+\cdots\left(\begin{array}{l}
m \\
m
\end{array}\right)\left(\begin{array}{c}
n \\
n-m
\end{array}\right) \\
& =\sum_{l=0}^{m}\left(\begin{array}{c}
m \\
l
\end{array}\right)\left(\begin{array}{c}
n \\
n-l
\end{array}\right)
\end{aligned}
$$

The last two lines of cyuation (B.13) follow from the fact that $\left(\begin{array}{c}m \\ l\end{array}\right)=0$ for $l>m$. Substituting coguation (B.13) into the second summation of equation (B.11) gives

$$
\begin{aligned}
\sum_{n=0}^{\infty}\left(\begin{array}{c}
m+n \\
n
\end{array}\right) \frac{\kappa_{f}^{n}}{n !} & =\sum_{n=0}^{\infty} \sum_{l=0}^{m}\left(\begin{array}{c}
m \\
l
\end{array}\right)\left(\begin{array}{c}
n \\
n-l
\end{array}\right) \frac{K_{f}^{n}}{n !} \\
& =\sum_{n=0}^{\infty} \sum_{l=0}^{m}\left(\begin{array}{l}
m \\
l
\end{array}\right) \frac{\kappa_{f}^{-l}}{l !} \cdot \frac{K_{f}^{n-l}}{(n-l) !}
\end{aligned}
$$

Noting that the factorial term $(n-l)$ ! is undefined for $n<l$ changes the range of the second summation from $(0 . x)$ to $(l, \infty)$. Redefining $n-l$ as $n$ gives

$$
\sum_{n=0}^{\infty}\left(\begin{array}{c}
m+n \\
n
\end{array}\right) \frac{h_{f}^{\prime}}{n !}==\sum_{l=0}^{m}\left(\begin{array}{c}
n \\
l
\end{array}\right) \frac{K_{f}^{\prime l}}{l !} \cdot \sum_{n=0}^{\infty} \frac{K_{f}^{n}}{n !}
$$




$$
=\exp K_{f} \sum_{l=0}^{m}\left(\begin{array}{c}
m \\
l
\end{array}\right) \frac{K_{f}^{-1}}{l !}
$$

Finally, substituting equation (B.15) into equation (B.1l) gives the desired re.ult. namely

$$
\mathbf{E}\left[\rho^{2 \pi m}\right]=\frac{m !}{\left(1+K_{f}\right)^{m}} \cdot \sum_{l=0}^{m}\left(\begin{array}{c}
m \\
l
\end{array}\right) \frac{K_{f}^{-l}}{l !}
$$

which can be evaluated easily since the number of terms in the summation is mual to the order of the Rician moment that is being calculated. 


\section{Appendix $\mathbf{C}$}

\section{Exponential Integral}

In the derivation of the pairwise error probability for the Rician fading channel it was necessary to evaluate an integral of the form

$$
I=\int_{0}^{\infty} x \exp \left[-\left(a x^{2}+b x\right)\right] d x
$$

('ompleting the square of the guadratic in the arguement of the exponential gives

$$
I=\exp \left(a c^{2}\right) \cdot \int_{0}^{\infty} x \exp \left[-a(x+c)^{2}\right] d x
$$

in which $c=\frac{b}{2 a}$. Sulstituting $z=\sqrt{a}(x+c)$ into equation (C.2) gives an expression of the form

$$
\begin{aligned}
I & =\exp \left(a c^{2}\right) \cdot \frac{1}{\sqrt{a}} \int_{\sqrt{a} \cdot c}^{\infty}\left(\frac{z}{\sqrt{a}}-c\right) \exp \left(-z^{2}\right) d z \\
& =\exp \left(a c^{2}\right) \cdot\left[\frac{1}{a} \int_{\sqrt{a} \cdot c}^{\infty} z \exp \left(-z^{2}\right) d z-\frac{c}{\sqrt{a}} \int_{\sqrt{a} \cdot c}^{\infty} \exp \left(-z^{2}\right) d z\right]
\end{aligned}
$$

Defining the first integral o: the righthand side of equation (C.3) as $I_{1}$ and making the substitution $"=z^{2}$ gives

$$
\begin{aligned}
I_{1} & =\frac{1}{2 a} \int_{a \cdot c^{2}}^{\infty} \exp \left(-c^{\prime}\right) d v \\
& =\frac{1}{2 a} \cdot \frac{1}{\exp \left(a c^{2}\right)}
\end{aligned}
$$


The second integral of equation (C.3) can be recognized as

$$
I_{2}=\frac{c \sqrt{\pi}}{2 \sqrt{a}} \operatorname{erfc}(c \sqrt{a})
$$

in which

$$
\operatorname{erfc}(x)=\frac{2}{\sqrt{\pi}} \int_{x}^{\infty} \exp \left(-z^{2}\right) d z
$$

Combining the results of equations (C.4) and (C.5) gives

$$
I=\frac{1}{2 a}-\frac{c \sqrt{\pi}}{2 \sqrt{a}} \exp \left(a c^{2}\right) \cdot \operatorname{erfc}(c \sqrt{a})
$$




\section{Appendix D}

\section{I and $Q$ Interuser Interference Analysis}

\section{D.1 Derivation of Interference}

This appendix derives the moments of interuser interference by treating the outputs of the $\mathrm{I}$ and $\mathrm{Q}$ channel correlators in Figure 2.8 as seperate random variables (i.e. as opposed to the complex analysis employed in Chapter 2). As will become evident, this analysis tends to be more cumbersome than the complex analysis.

Examining the output of the correlator in the $f^{\text {th }}$ symbol interval (i.e. see equation (2.12)), the I channel can be expressed as

$$
\begin{aligned}
Y_{l_{v}}= & \sqrt{\frac{2}{T}} \int_{0}^{T} r(t) a^{i}(t) \cos \left(\omega_{c} t\right) d t \\
= & 2 \frac{\sqrt{E_{s}}}{T} \int_{0}^{T} \sum_{k=1}^{k}\left[a^{k}\left(t-\tau^{k}\right) b^{k}\left(t-\tau^{k}\right) \cos \left(\omega_{c} t+\theta^{k}(t)+\beta^{k}\right)\right] a^{i}(t) \cos \left(\omega_{c} t\right) d t \\
& \quad+\sqrt{\frac{2}{T}} \int_{0}^{T} n(t) a^{i}(t) \cos \left(\omega_{c} t\right) d t \\
= & \sqrt{E_{s}}\left\{b_{p}^{i} \cos \left(\theta_{p}^{i}\right)+\right.
\end{aligned}
$$




$$
\left.\frac{1}{T} \sum_{\substack{k=1 \\ k \neq i}}^{h}\left[b_{p-1}^{k} R_{k, 4}\left(\tau^{k}\right) \cos \left(\theta_{p-1}^{k}+\beta^{k}\right)+b_{p}^{k} \hat{R}_{k, i}\left(\tau^{k}\right) \cos \left(\theta_{p}^{k}+j^{k}\right)\right]\right\}+\eta_{\text {, }}^{i}
$$

in which $\eta_{I}$ is a sample of a Gaussian process with variance $N_{0} / 2$. Similarly, the output the the quadrature correlator is

$$
\begin{aligned}
& Y_{Q_{0}}^{3}= \sqrt{\frac{2}{T}} \int_{0}^{T} r(t) a^{i}(t) \sin \left(\omega_{c} t\right) d t \\
&= 2 \frac{\sqrt{E_{s}}}{T^{\prime}} \int_{0}^{T} \sum_{k=1}^{K}\left[a^{k}\left(t-\tau^{k}\right) b^{k}\left(t-\tau^{k}\right) \cos \left(\omega_{c} t+\theta^{k}(t)+s^{k}\right)\right] a^{i}(t) \sin \left(\omega_{c} t\right) d t \\
& \quad+\sqrt{\frac{2}{T}} \int_{0}^{T} n(t) a^{2}(t) \sin \left(\omega_{c} t\right) d t \\
&= \sqrt{E_{s}}\left\{b_{p}^{i} \sin \left(\theta_{p}^{i}\right)+\right. \\
&\left.\frac{1}{T} \sum_{\substack{k=1 \\
k \neq i}}^{K}\left[b_{p-1}^{k} R_{k, i}\left(\tau^{k}\right) \sin \left(\theta_{p-1}^{k}+\beta^{k}\right)+b_{p}^{k} \dot{R}_{k, \mathrm{~s}}\left(\tau^{k}\right) \sin \left(\theta_{p}^{k}+\beta^{k}\right)\right]\right\}+\eta_{Q_{p}}^{i}
\end{aligned}
$$

The results of equations (D.1) and and (D.2) are valid if one assumes that $w_{r} \gg T^{-1}$. in which case the higher order frequency terms are negligible. Furthermore it. has been assumed as before that $\tau^{2}=0$ without loss of generality (note that these equations are analogous to equation (2.13)). In equation (D.1) the term $\sqrt{E_{s}} b_{p}^{\mathrm{s}} \cos \left(\theta_{p}^{z}\right)$ is the signal component of the in-phase channel, whereas in equation (D.2) $\sqrt{E_{s}} b_{p}^{i} \sin \left(\theta_{p}^{2}\right)$ is ihe signal component of the quadrature channel (i.e the real and imaginary parts of $\left.X_{p}^{t}\right)$. The expressions within the summations of explations (D.1) and (D.2) are the in phase and quadrature components of the interuser interference. Thus one can define the energy normalized in-phase and puadrature: components of the interuser interference in the $p^{\text {th }}$ symbol of the $i^{\text {th }}$ user as $z_{y_{y}}$ ard 
$z_{Q_{p}}^{\prime}$ respectively

$$
\begin{aligned}
& z_{i_{p}}^{i}=\frac{1}{T} \sum_{\substack{k=1 \\
k \neq 1}}^{K}\left[b_{p-1}^{k} R_{k, i}\left(\tau^{k}\right) \cos \left(\theta_{p-1}^{k}+\beta^{k}\right)+b_{p}^{k} \hat{R}_{k, i}\left(\tau^{k}\right) \cos \left(\theta_{p}^{k}+\beta^{k}\right)\right] \\
& z_{Q_{p}}^{\prime}=\frac{1}{T} \sum_{\substack{k=1 \\
k \neq i}}^{K}\left[b_{p-1}^{k} R_{k, i}\left(\tau^{k}\right) \sin \left(\theta_{p-1}^{k}+\beta^{k}\right)+b_{p}^{k} \hat{R}_{k, i}\left(\tau^{k}\right) \sin \left(\theta_{p}^{k}+\beta^{k}\right)\right]
\end{aligned}
$$

Thus equations (D.1) and and (D.2) become

$$
\begin{aligned}
& Y_{I_{p}}^{i}=\sqrt{E_{s}}\left\{b_{p}^{i} \cos \left(\theta_{p}^{i}\right)+z_{I_{p}}^{i}\right\}+\eta_{I_{p}}^{i} \\
& Y_{Q_{p}}=\sqrt{E_{\varepsilon}}\left\{b_{p}^{i} \sin \left(\theta_{p}^{i}\right)+z_{Q_{p}}^{i}\right\}+\eta_{Q_{p}}^{i}
\end{aligned}
$$

The continuous-time partial cross-correlation functions $R_{k, i}\left(\tau^{k}\right)$ and $\hat{R}_{k, i}\left(\tau^{k}\right)$ are defince in equations (2.11) and (2.15) of Chapter 3. Expanding the expression for $z_{l_{p}}$ in rquation (D.3) one obtains

$$
\begin{aligned}
& z_{i_{p}}^{i}=\frac{1}{T} \sum_{\substack{k=1 \\
k \neq i}}^{K}\{ {\left[\cos \left(\theta_{p-1}^{k}\right) \cos \left(\beta^{k}\right)-\sin \left(\theta_{p-1}^{k}\right) \sin \left(\beta^{k}\right)\right] b_{p-1}^{k} R_{k, z}\left(\tau^{k}\right) } \\
&\left.+\left[\cos \left(\theta_{p}^{k}\right) \cos \left(\beta^{k}\right)-\sin \left(\theta_{p}^{k}\right) \sin \left(\beta^{k}\right)\right] b_{p}^{k} \hat{R}_{k, i}\left(\tau^{k}\right)\right\} \\
&=\frac{1}{T} \sum_{\substack{k=1 \\
k \neq 1}}^{K}\left\{\left[b_{p-1}^{k} \cos \left(\theta_{p-1}^{k}\right) R_{k, 1}\left(\tau^{k}\right)+b_{p}^{k} \cos \left(\theta_{p}^{k}\right) \hat{R}_{k, 1}\left(\tau^{k}\right)\right] \cos \left(\beta^{k}\right)\right. \\
&\left.-\left[b_{p-1}^{k} \sin \left(\theta_{p-1}^{k}\right) R_{k, s}\left(\tau^{k}\right)+b_{p}^{k} \sin \left(\theta_{p}^{k}\right) \hat{R}_{k, 1}\left(\tau^{k}\right)\right] \sin \left(\beta^{k}\right)\right\}
\end{aligned}
$$

Similarily, the expression for $\tilde{Q}_{Q_{p}}^{i}$ in equation (D.4) gives

$$
\begin{aligned}
z_{Q_{p}}^{\prime}=\frac{1}{T} \sum_{\substack{k=1 \\
k \neq 1}}^{K}\{ & \left\{\sin \left(\theta_{p-1}^{k}\right) \cos \left(\beta^{k}\right)+\cos \left(\theta_{p-1}^{k}\right) \sin \left(\beta^{k}\right)\right] b_{p-1}^{k} R_{k, 1}\left(\tau^{k}\right) \\
+ & \left.+\left[\sin \left(\theta_{p}^{k}\right) \cos \left(\beta^{k}\right)+\cos \left(\theta_{p}^{k}\right) \sin \left(3^{k}\right)\right] b_{p}^{k} \hat{R}_{k, i}\left(\tau^{k}\right)\right\}
\end{aligned}
$$




$$
\begin{aligned}
=\frac{1}{T} \sum_{\substack{k=1 \\
k \neq i}}^{K}\left\{\left[b_{p-1}^{k} \sin \left(\theta_{p-1}^{k}\right) R_{k, i}\left(\tau^{k}\right)+b_{p}^{k} \sin \left(\theta_{p}^{k}\right) \hat{R}_{k, i}\left(\tau^{k}\right)\right] \cos \left(\beta^{k}\right)\right. \\
\left.\quad+\left[b_{p-1}^{k} \cos \left(\theta_{p-1}^{k}\right) R_{k, 1}\left(\tau^{k}\right)+b_{p}^{k} \cos \left(\theta_{p}^{k}\right) \hat{R}_{k, 1}\left(\tau^{k}\right)\right] \sin \left(\beta^{k}\right)\right\}
\end{aligned}
$$

Define $E_{p}^{k}$ as

$$
\begin{aligned}
E_{p}^{k} & =b_{p-1}^{k} \cos \left(\theta_{p-1}^{k}\right) R_{k, i}\left(\tau^{k}\right)+b_{p}^{k} \cos \left(\theta_{p}^{k}\right) \hat{R}_{k, i}\left(\tau^{k}\right) \\
& =x_{l_{p-1}}^{k} R_{k, i}\left(\tau^{k}\right)+x_{l_{p}}^{k} \hat{R}_{k, i}\left(\tau^{k}\right)
\end{aligned}
$$

and $F_{p}^{k}$ as

$$
\begin{aligned}
F_{p}^{k} & =b_{p-1}^{k} \sin \left(\theta_{p-1}^{k}\right) R_{k, i}\left(\tau^{k}\right)+b_{p}^{k} \sin \left(\theta_{p}^{k}\right) \hat{R}_{k, 1}\left(\tau^{k}\right) \\
& =x_{Q_{p-1}}^{k} R_{k, i}\left(\tau^{k}\right)+x_{Q_{p}}^{k} \hat{R}_{k, i}\left(\tau^{k}\right)
\end{aligned}
$$

in which $x_{l_{p}}^{k}$ and $x_{Q_{p}}^{k}$ are the in-phase and quadrature components of the $p^{\text {th }}$ transmitted symbol of the $k^{\text {th }}$ interferer (i.e. $x_{p}^{k}$ ). Using equations (D.9) and (D.10) one can rewrite equations (D.T) and (D.8) as

$$
\begin{aligned}
& z_{I_{p}}^{k}=\frac{1}{T} \sum_{\substack{k=1 \\
k \neq i}}^{K}\left[E_{p}^{k} \cos \left(\beta^{k}\right)-F_{p}^{k} \sin \left(\beta^{k}\right)\right] \\
& z_{Q_{p}}^{k}=\frac{1}{T} \sum_{\substack{k=1 \\
k \neq i}}^{K}\left[F_{p}^{k} \cos \left(\beta^{k}\right)+E_{p}^{k} \sin \left(\beta^{k}\right)\right]
\end{aligned}
$$

\section{D.2 Evaluation of the Moments of the Interuser Interference}

The moments of the interuser interference can now be evaluated in in manner similar to that derived for the complex analysis of $A$ ppendix $A$. Rewriting aduation $(\Lambda .1)$ 
in terms of its real and imaginary components gives

$$
\begin{aligned}
E\left[v^{m}\right] & =\mathbf{E}\left[\sum_{p=0}^{N-1} d_{I_{p} z_{I_{p}}}+d_{Q_{p}} z_{Q_{p}}\right]^{m} \quad \text { for } \mathrm{m}=0,1,2 \cdots N_{m} \\
& =\mathrm{E}\left[d_{I_{0}} z_{I_{0}}+d_{Q_{1}} z_{Q_{1}}+d_{I_{0}} z_{I_{0}}+d_{Q_{1}} z_{Q_{1}}+\cdots+d_{I_{N-1}} z_{I_{N-1}}+d_{Q_{N-1}} z_{Q_{N-1}}\right]^{m}
\end{aligned}
$$

The final expression of equation (D.13) is the term of interest. Following the derivation of Appendix A, equation (D.13) can be expressed as a multinomial expansion giving

$$
\begin{aligned}
& \mathbf{E}\left[v^{m}\right]=\sum \frac{m !}{i_{0} ! q_{0} ! i_{1} ! q_{1} ! \cdots i_{N-1} ! q_{N-1} !} \\
& \left(d_{I_{0}}\right)^{i_{0}}\left(d_{Q_{0}}\right)^{30}\left(d_{I_{1}}\right)^{i_{1}}\left(d_{Q_{1}}\right)^{q_{1}} \cdots\left(d_{I_{N-1}}\right)^{i_{N-1}}\left(d_{Q_{N-1}}\right)^{q_{N-1}} \\
& \cdot\left(z_{I_{0}}\right)^{10}\left(z_{Q_{0}}\right)^{p^{\circ}}\left(z_{I_{1}}\right)^{i_{1}}\left(z_{Q_{1}}\right)^{q_{1}} \cdots\left(z_{I_{N-1}}\right)^{z_{N-1}}\left(z_{Q_{N-1}}\right)^{q_{N-1}} \\
& =\sum\left[n ! \cdot \prod_{p=0}^{N-1} \frac{d_{l_{0}}^{i_{0}} d_{Q_{0}}^{q_{0}}}{i_{p} ! q_{p} !} \mathbf{E}\left\{\mathcal{Z}^{\prime}\left(z_{l}, z_{Q}\right)\right\}\right]
\end{aligned}
$$

in which the summation is over all possible combinations of $i_{0}+q_{0}+i_{1}+q_{1}+\cdots+$ $i_{N-1}+q_{N-1}$ that sum to $m$. Furthermore, since for a given error sequence $d$, the function $D\left(d_{I_{p}}, d_{Q_{p}}\right)$ is a constant, the expectation when evaluating equation (D.14) is taken over the product of the IUI terms

$$
\mathcal{Z}^{\prime}\left(z_{1}, z_{Q}\right)=\prod_{p=0}^{N-1}\left(z_{l_{p}}\right)^{i_{p}}\left(z_{Q_{p}}\right)^{q_{p}}
$$

I'sing the definition of $\mathcal{Z}^{\prime}\left(z l, z_{Q}\right)$ and substituting into it the definitions of $z_{I_{p}}$ and $\varepsilon_{p}$ (see equations (D.11) and (D.12)) one obtains for any interferer $k$

$$
\begin{aligned}
& z^{\prime}\left(z_{1}, z_{Q}\right)=\prod_{p=0}^{N-1}\left\{\frac{1}{T^{i_{p}+q_{p}}}\left[E_{p} \cos (\beta)-F_{p} \sin (\beta)\right]^{i_{p}}\right. \\
&\left.\cdot\left[F_{p} \cos (\beta)+E_{p} \sin (\beta)\right]^{q_{p}}\right\}
\end{aligned}
$$


Expanding equation (D.16) gives

$$
\begin{aligned}
& \mathcal{Z}^{\prime}\left(z_{l}, z_{Q}\right)=\prod_{p=0}^{N-1}\left\{\sum_{a_{p}=0}^{i_{p}}\left(\begin{array}{c}
i_{p} \\
a_{p}
\end{array}\right)(-1)^{i_{p}-a_{p}}[\cos (\beta)]^{a_{p}}[\sin (\beta)]^{p_{p}-a_{p}} E_{p}^{a_{r}} F_{p}^{i_{p}-a_{p}}\right. \\
& \left.\cdot \sum_{b_{p}=0}^{q_{p}}\left(\begin{array}{l}
q_{p} \\
b_{p}
\end{array}\right)[\sin (\beta)]^{b_{p}}[\cos (\beta)]^{q_{p}-b_{p}} E_{p}^{b_{p}} F_{p}^{q_{p}-b_{p}}\right\} \\
& =\prod_{p=0}^{N-1}\left\{\sum_{a_{p}=0}^{i_{p}} \sum_{b_{p}=0}^{q_{p}}\left(\begin{array}{l}
i_{p} \\
a_{p}
\end{array}\right)\left(\begin{array}{l}
q_{p} \\
b_{p}
\end{array}\right)(-1)^{i_{p}-a_{p}}[\cos (\beta)]^{a_{p}+q_{p}-b_{p}}[\sin (\beta)]^{i_{p}-a_{p}+b_{p}}\right. \\
& \text { - } \left.E_{p}^{a_{p}+b_{p}} F_{p}^{i_{p}+q_{p}-a_{p}-b_{p}}\right\} \\
& =\sum_{a_{0}=0}^{i_{0}} \sum_{b_{0}=0}^{q_{0}} \sum_{a_{1}=0}^{i_{1}} \sum_{b_{1}=0}^{q_{1}} \cdots \sum_{a_{N-1}=0}^{i_{N-1}} \sum_{b_{N-1}=0}^{q_{N}-1} \\
& \left(\begin{array}{l}
i_{0} \\
a_{0}
\end{array}\right)\left(\begin{array}{l}
q_{0} \\
b_{0}
\end{array}\right)\left(\begin{array}{l}
i_{1} \\
a_{1}
\end{array}\right)\left(\begin{array}{l}
q_{1} \\
b_{1}
\end{array}\right) \cdots\left(\begin{array}{l}
i_{N-1} \\
a_{N-1}
\end{array}\right)\left(\begin{array}{l}
q_{N-1} \\
b_{N-1}
\end{array}\right) \\
& \cdot(-1)^{i_{0}+i_{1}+\cdots+i_{N-1}-a_{0}-a_{1}-\cdots-a_{N-1}} \\
& \cdot \prod_{p=0}^{N-1}\left\{[\cos (\beta)]^{a_{p}+q_{p}-b_{p}}[\sin (\beta)]^{\alpha_{p}-a_{p}+b_{p}}\right\} \\
& \cdot \prod_{p=0}^{N-1}\left\{F_{p}^{a_{p}+b_{p}} F_{p}^{i_{p}+q_{p}-a_{p}-b_{p}}\right\}
\end{aligned}
$$

Taking the expectation of equation (D.17) results in

$$
\begin{aligned}
\mathbf{E}\left[\mathcal{Z}^{\prime}\left(z_{1}, z_{Q}\right)\right]= & \sum_{a_{0}=0}^{i_{0}} \sum_{b_{0}=0}^{q_{0}} \sum_{a_{1}=0}^{i_{1}} \sum_{b_{1}=0}^{q_{1}} \ldots \sum_{a_{N-1}=0}^{i_{N-1}} \sum_{b_{N-1}=0}^{q_{N-1}} \\
& \cdot\left(\begin{array}{c}
i_{0} \\
a_{0}
\end{array}\right)\left(\begin{array}{l}
q_{0} \\
b_{0}
\end{array}\right)\left(\begin{array}{l}
i_{1} \\
a_{1}
\end{array}\right)\left(\begin{array}{l}
q_{1} \\
b_{1}
\end{array}\right) \cdots\left(\begin{array}{c}
i_{N-1} \\
a_{N-1}
\end{array}\right)\left(\begin{array}{l}
q_{N-1} \\
b_{N-1}
\end{array}\right) \\
& \cdot(-1)^{i_{0}+1_{1}+\cdots+i_{N-1}-a_{0}-a_{1}-\cdots-a_{N-1}} \\
& \cdot \mathbf{E}\left\{\prod_{p=0}^{N-1}\left([\cos (\beta)]^{a_{p}+q_{p}-b_{p}}[\sin (y)]^{p_{p}-a_{p}+b_{p}}\right)\right\} \\
& \cdot \mathbf{E}\left\{\prod_{p=0}^{N-1}\left(E_{p}^{a_{p}+b_{p}} F_{p}^{a_{p}+q_{p}-a_{p}-b_{p}}\right)\right\}
\end{aligned}
$$

Note that in equation (D.18) it is not obvious that the expectation is independent 
of the random variable $\beta$. This is another disadvantage of the $I$ and $Q$ approach to analyzing the moments of the interuser interference, as opposed to the complex analysis of Appendix A. In the calculation of the expectation, the fact that the rantom variable $\beta$ is independent of the random variables $E^{p}$ and $F^{p}$ has been exploited. Furthermore it has been assumed that $\beta$ is constant over the length of the sequences of interest. This is valid if $\phi_{k}, \omega_{c}$ and $\tau_{k}$ do not vary significantly with time (sce equation (2.9)). Thus the problem reduces to determining the expectation of the two expressions on the righthand side of equation (D.18). Define the first expectation involving the trigonometric functions sine and $\operatorname{cosine}$ as $\mathcal{C}(\cos \beta \cdot \sin \beta)$. In $\Lambda$ ppendix $\mathrm{F} \mathcal{C}(\cos \beta, \sin \beta)$ is shown to be

$$
E\left\{[\cos (\beta)]^{r}[\sin (, \beta)]^{\prime}\right\}= \begin{cases}\frac{\left(\begin{array}{c}
r \\
r / 2
\end{array}\right) \cdot\left(\begin{array}{c}
s \\
s / 2
\end{array}\right)}{(r+o) / 2 \cdot\left(\begin{array}{c}
(r+s) / 2 \\
s / 2
\end{array}\right)} & \text { for } \mathrm{r} \text { and s even } \\
0 & \text { for } \mathrm{r} \text { or } \mathrm{s} \text { odd }\end{cases}
$$

in winich

$$
\begin{aligned}
& r=\sum_{p=0}^{N-1}\left[a_{p}+q_{p}-b_{p}\right] \\
& s=\sum_{p=0}^{N-1}\left[i_{p}-a_{p}+b_{p}\right]
\end{aligned}
$$

The expectation of the function of the random varibles $E_{p}$ and $F_{p}$ can be evaluated using the definitions of equations (D.9) and (D.10). Defining $\mathcal{F}\left(E_{p}, F_{p}\right)$ as the final product in equation (D.18) allows the analysis to continue as per equation (A.11) of Appendix A. 


\section{Appendix E}

\section{Correlation Moments of Common}

\section{Constellations}

From equation (A.14) it is required to determine the expectation defined by

$$
\begin{aligned}
& \mathcal{X}\left(x_{l_{p}}^{k}, x_{Q_{p}}^{k}\right)=E\left\{\left(x_{I_{-1}}^{k}\right)^{e_{0}}\left(x_{Q_{-1}}^{k}\right)^{f_{0}} \prod_{p=0}^{N-2}\left[\left(x_{l_{p}}^{k}\right)^{u_{p}-e_{p}+e_{p+1}}\left(x_{Q_{p}}^{k}\right)^{v_{p}-f_{p}+f_{p+1}}\right]\right. \\
& \left.\cdot\left(x_{I_{N-1}}^{k}\right)^{u_{N-1}-e_{N-1}}\left(x_{Q_{N-1}}^{k}\right)^{u_{N-1-e N-1}}\right\} \\
& =E\left\{\left(. c_{-1}\right)^{i-1}\left(x_{Q_{-1}}\right)^{q-1}\left(x_{I_{0}}\right)^{x_{0}}\left(x_{Q_{0}}\right)^{70} \cdots\left(x_{I_{N-1}}\right)^{I_{N-1}}\left(x_{Q_{N-1}}\right)^{q_{N-1}}\right\}
\end{aligned}
$$

Note that the interferer superscript in the second line has been dropped without any loss of generality. Furthermore in equation (E.1)

$$
\begin{aligned}
i_{-1} & =e_{-1} \\
q_{-1} & =f_{-1} \\
i_{p} & =u_{p}-\epsilon_{p}+e_{p+1} \text { for } \mathrm{p}=0,1, \cdots, N_{-2} \\
q_{p} & =v_{p}-f_{p}+f_{p+1} \text { for } \mathrm{p}=0,1, \cdots N-2 \\
& \text { and } \\
i_{N-1} & =u_{N-1}-e_{N-1}
\end{aligned}
$$




$$
\tau_{N-1}=v_{N-1}-f_{N-1}
$$

The signal points in equation (E.2) are chosen from the constellation under consideration according to the output of the convolutional coder or the trellis coder. Though this produces some correlation between adjacent signal points, due to the symmetry of the constellations and the coder, the in-phase and quadrature components are chosen with equal steady state prubabilities in any two symbol intervals. Thus for the purposes of averaging, the symbols can be treated as being independent from one symbol period to the next. In this case equation (E.1) can be rewritten as

$$
x^{\prime}\left(x_{l_{p}}^{k}, x_{Q_{p}}^{k}\right)=\prod_{p=-1}^{N-1}\left[E\left\{\left(x_{l_{p}}\right)^{p_{p}}\left(x_{Q_{p}}\right)^{q_{p}}\right\}\right]
$$

Thus the problem reduces to calculating an expectation of the form $E\left\{\left(x_{I_{p}}\right)^{m}\left(x_{Q_{p}}\right)^{n}\right\}$ for $m$ and $n>0$.

\section{E.1 2-PSK Constellation}

Choose an arbitrary 2-PSK constellation with anti-podal signal points at $\left(\frac{1}{\sqrt{2}}, \frac{1}{\sqrt{2}}\right)$ and $\left(\frac{-1}{\sqrt{2}}, \frac{-1}{\sqrt{2}}\right)$. It then follows by inspection that

$$
\mathbf{E}\left\{\left(\boldsymbol{r}_{l_{p}}\right)^{m}\left(x_{Q_{p}}\right)^{n}\right\}=\left(\frac{1}{\sqrt{2}}\right)^{m+n}
$$

if $m+n$ is even. otherwise the expectation is zero.

\section{E.2 4-PSK Constellation}

Figure E.1 illustrates the 4-PSK consteilation with the in-phase and quadrature components appropriately labelled. Due to the symmetry of the constellation, if $m$ or $n$ is odd. then

$$
\mathbf{E}\left\{\left(x_{l_{p}}\right)^{m}\left(x_{Q_{p}}\right)^{n}\right\}=0
$$


If both $m$ and $n$ are even then the expectation takes on the form

$$
\begin{aligned}
E\left\{\left(x_{l_{p}}\right)^{2 m}\left(x_{Q_{r}}\right)^{2 n}\right\} & =\frac{1}{4}\left[\left(\frac{1}{\sqrt{2}}\right)^{2 m}\left(\frac{1}{\sqrt{2}}\right)^{2 n}+\cdots+\left(\frac{-1}{\sqrt{2}}\right)^{2 m}\left(\frac{-1}{\sqrt{2}}\right)^{2 n}\right] \\
& =\frac{1}{2^{m+n}}
\end{aligned}
$$

\section{E.3 8-PSK Constellation}

The 8-PSK constellation with appropriately labelled in-phase and quadrature components is illustrated in Figure E.2. As in the case of the 4.PSK constellation, if $m$ or $n$ is odd. then

$$
\mathbf{E}\left\{\left(x_{p}\right)^{m}\left(x_{Q p}\right)^{n}\right\}=0
$$

Moreover if both $m$ and $n$ are even and nonzero, then as in the 4-PSK case

$$
\begin{aligned}
\mathbf{E}\left\{\left(x_{I_{p}}\right)^{2 m}\left(x_{Q_{p}}\right)^{2 n}\right\} & =\frac{1}{8}\left[\left(\frac{1}{\sqrt{2}}\right)^{2 m}\left(\frac{1}{\sqrt{2}}\right)^{2 n}+\cdots+\left(\frac{-1}{\sqrt{2}}\right)^{2 n}\left(\frac{-1}{\sqrt{2}}\right)^{2 n}\right] \\
& =\frac{1}{8}\left[4 \frac{1}{2^{m}} \frac{1}{2^{n}}\right] \\
& =\frac{1}{2^{m+n+1}}
\end{aligned}
$$

Furthermore, if $\boldsymbol{n}$ is zero then

$$
\begin{aligned}
\mathbf{E}\left\{\left(x_{I_{p}}\right)^{2 m}\right\} & =\frac{1}{x}\left[1+1+4 \times \frac{1}{2^{m}}\right] \\
& =\frac{2^{m-1}+1}{2^{2 n+1}}
\end{aligned}
$$

A similar analysis holds for $\mathbf{E}\left\{\left(x_{Q_{p}}\right)^{2 n}\right\}$ if $m=0$.

\section{E.4 16-PSK Constellation}

The 16-PSK constellation with appropriately labelled in-phase and quadrature components is illustrated in Figure E.3. As in the case of the 4.PSK constellation. if "r 
or $n$ is odd, then

$$
E\left\{\left(x_{l_{p}}\right)^{m}\left(x_{Q_{p}}\right)^{n}\right\}=0
$$

If loth $m$ and $n$ are even and nonzero, then averaging over each of the 16 signal constellation points gives

$$
\begin{aligned}
\mathbf{E}\left\{\left(r_{p}\right)^{2 m}\left(x_{Q_{p}}\right)^{2 n}\right\}= & \frac{1}{16}\left[1 \times\left(\frac{1}{\sqrt{2}}\right)^{2 m}\left(\frac{1}{\sqrt{2}}\right)^{2 n}+\right. \\
& 4 \times\left[\left(\frac{\sqrt{2}+1}{2 \sqrt{2}}\right)^{m} \cdot\left(\frac{\sqrt{2}-1}{2 \sqrt{2}}\right)^{n}+\right. \\
& \left.\left.\left(\frac{\sqrt{2}+1}{2 \sqrt{2}}\right)^{n} \cdot\left(\frac{\sqrt{2}-1}{2 \sqrt{2}}\right)^{m}\right]\right] \\
= & \frac{1}{16}\left[4 \cdot\left(\frac{1}{2}\right)^{m+n}+\frac{4}{(2 \sqrt{2})^{m+n}} \cdot\left[(\sqrt{2}+1)^{m}(\sqrt{2}-1)^{n}+\right.\right. \\
& \left.\left.(\sqrt{2}+1)^{n}(\sqrt{2}-1)^{m}\right]\right] \\
= & \frac{1}{2^{m+n+2}}+\frac{1}{4 \cdot(2 \sqrt{2})^{m+n}} \cdot\left[(\sqrt{2}+1)^{m-n}+(\sqrt{2}-1)^{m-n}\right] \\
& \text { for } m>n
\end{aligned}
$$

If $n=0$, then the expectation takes on the following form

$$
\begin{aligned}
\mathbf{E}\left\{\left(x_{1},\right)^{2 m}\right\} & =\frac{1}{16}\left[2(1)+1 \times\left(\frac{1}{\sqrt{2}}\right)^{2 m}+4 \times\left[\left(\frac{\sqrt{2}+1}{2 \sqrt{2}}\right)^{m}+\left(\frac{\sqrt{2}-1}{2 \sqrt{2}}\right)^{m}\right]\right] \\
& =\frac{1}{16}\left[2+\frac{1}{2^{m-2}}+4 \times\left[\frac{(\sqrt{2}+1)^{m}+(\sqrt{2}-1)^{m}}{(2 \sqrt{2})^{m}}\right]\right]
\end{aligned}
$$

1 similar analy isis holds for $\mathbf{E}\left\{\left(x_{Q_{p}}\right)^{2 n}\right\}$ if $m=0$. 


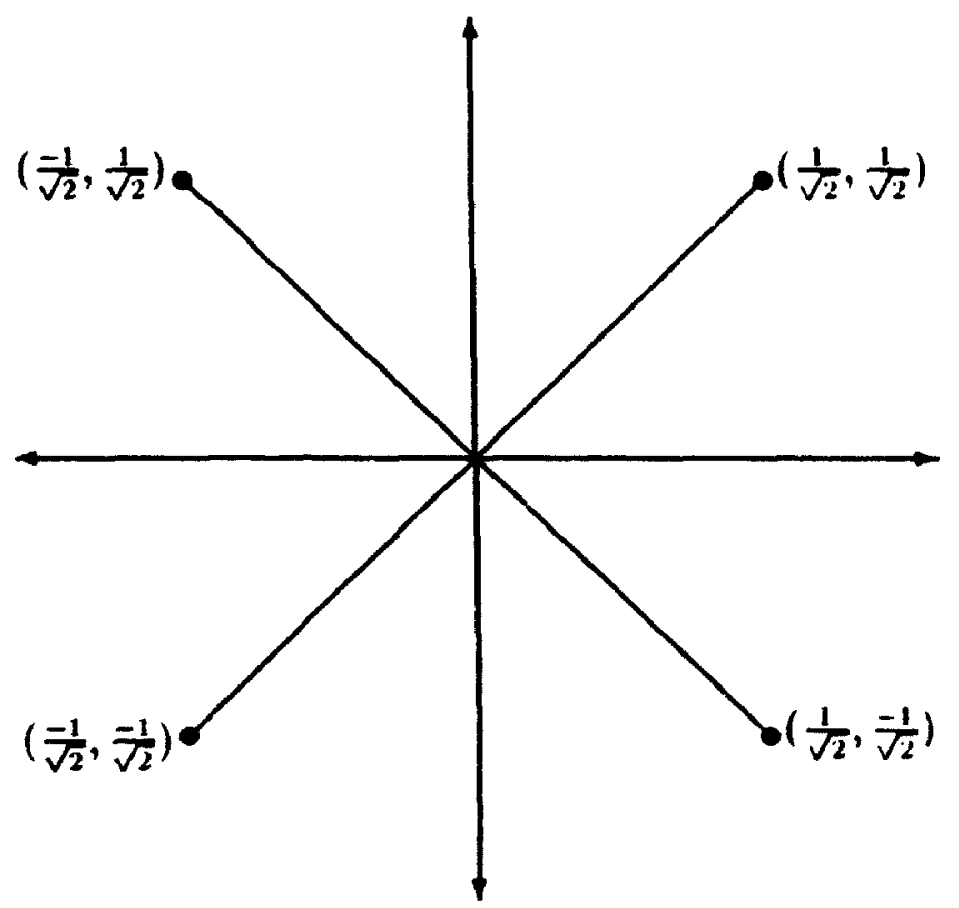

Figure E.1: Signal constellation of 4-PSK with labelled 1 and $Q$ components 


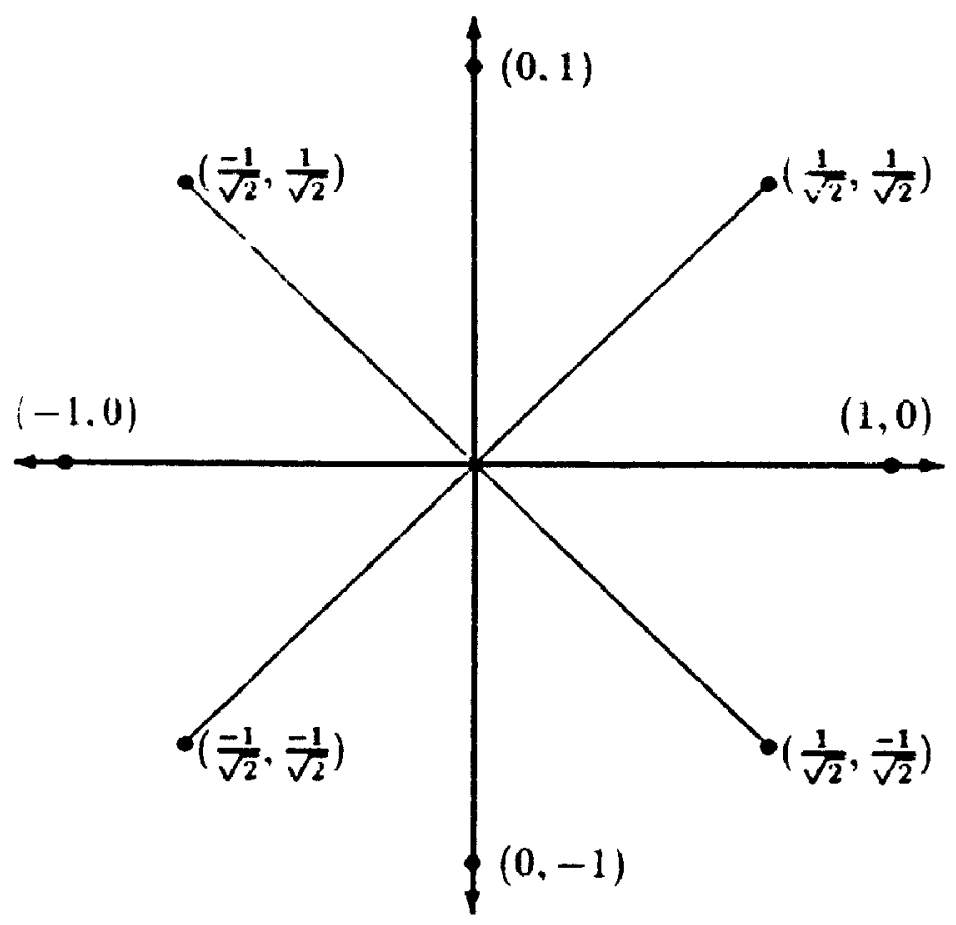

Figure l..2: \&-PSK signal constellation with labelled I and Q components. 


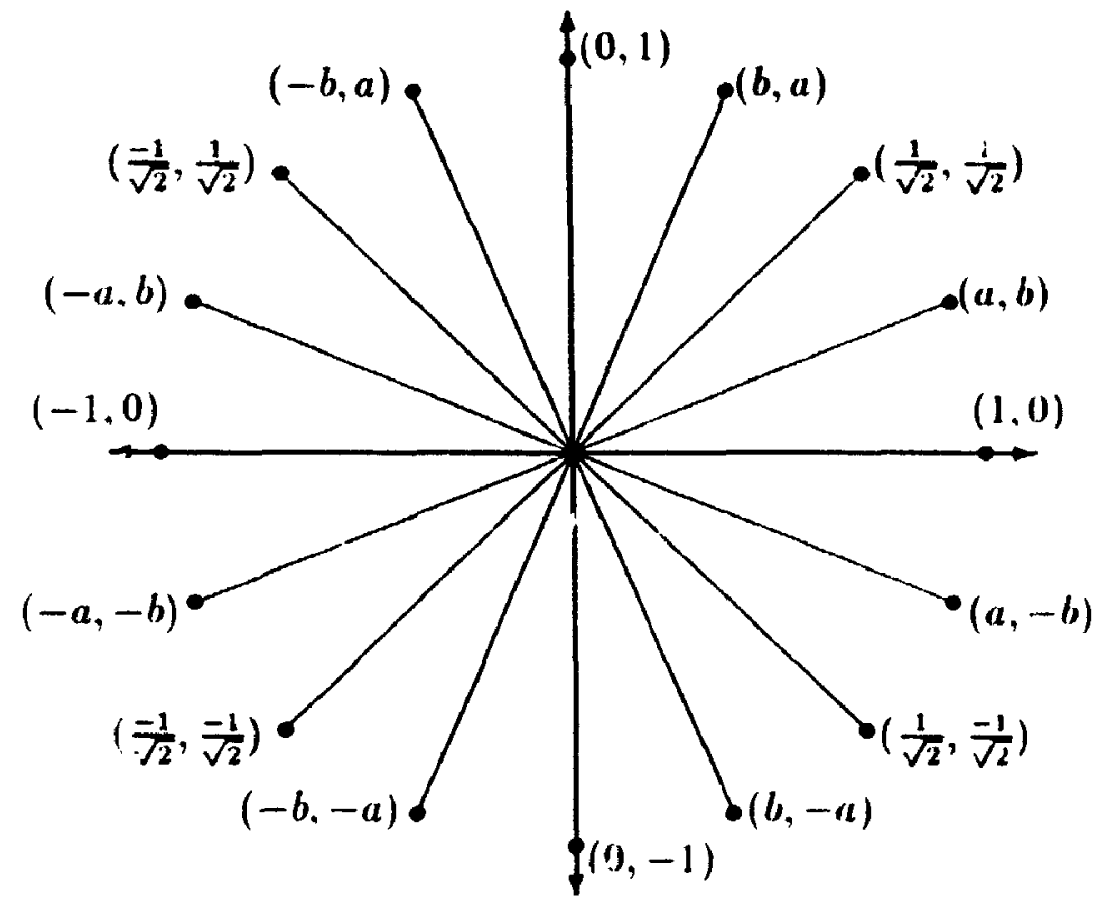

$$
\begin{aligned}
& a=\sqrt{\frac{\sqrt{2}+1}{2 \sqrt{2}}} \\
& b=\sqrt{\frac{\sqrt{2}-1}{2 \sqrt{2}}}
\end{aligned}
$$

Figure E.3: 16-PSK : ignal constellation with labelled I and $Q$ components. 


\section{Appendix $\mathbf{F}$}

\section{Moments of Product of Cosine and Sine Functions}

Consider the expectation

$$
I=\mathbf{E}\left\{[\cos (x)]^{m}[\sin (x)]^{n}\right\}=\frac{\mathrm{i}}{2 \pi} \int_{0}^{2 \pi}[\cos (x)]^{m}[\sin (x)]^{n} d x
$$

From standard integration tables [117] or by using integration by parts

$$
\begin{aligned}
& I=\left\{\begin{array}{l}
\left.\frac{\cos ^{m-1} x \cdot \sin ^{n+1} x}{m+n}\right|_{0} ^{2 \pi}+\frac{m-1}{m+n} \int_{0}^{2 \pi} \cos ^{m-2} x \cdot \sin ^{n} x d x \\
\left.\frac{-\sin ^{r-1} x \cdot \cos ^{m+1} x}{m+n}\right|_{0} ^{2 \pi}+\frac{m-1}{m+n} \int_{0}^{2 \pi} \cos ^{m} x \cdot \sin ^{n-2} x d x
\end{array}\right. \\
& =\left\{\begin{array}{l}
\frac{m-1}{m+n} \int_{0}^{2 \pi} \cos ^{m-2} x \cdot \sin ^{n} x d x \\
\text { or } \frac{m-1}{m+n} \int_{0}^{2 \pi} \cos ^{m} x \cdot \sin ^{n-2} x d x
\end{array}\right.
\end{aligned}
$$

If $m$ or $n$ is odd. repeated application of equation (F.2) results in an integral that has an integrand of a cosine or a sine function raised to an odd exponent, which integrates to zero. If both $m$ and $n$ are even, define

$$
I_{2}=\frac{1}{3 \pi} \int_{0}^{2 \pi}[\cos (x)]^{2 m}[\sin (x)]^{2 n} d x
$$


repeated application of the results of equation (F.2) to equation (F.3) gives

$$
I=\frac{2 m-1}{2 n+2 m} \cdot \frac{2 m-3}{2 n+2 m-2} \cdots \frac{3}{4+2 n} \cdot \frac{1}{2+2 n} \cdot \frac{2 n-1}{2 n} \cdot \frac{2 n-3}{2 n-2} \cdots \frac{3}{4} \cdot \frac{1}{2}
$$

Multiplying the numerator and denominator of $I$ by $2^{m+n} m ! n !$ gives

$$
\begin{aligned}
I & =\frac{(2 m) !(2 n) !}{2^{m+n} m ! n !} \times \frac{1}{2^{m+n}(m+n) !} \\
& =\frac{\left(\begin{array}{c}
2 m \\
m
\end{array}\right)\left(\begin{array}{c}
2 n \\
n
\end{array}\right)}{4^{m+n}\left(\begin{array}{c}
m+n \\
n
\end{array}\right)}
\end{aligned}
$$

Finally, the result quoted in equation (D.19) follows from equation (F.5). 


\section{Appendix G}

\section{Dependence of $z_{I_{p}}$ and $z_{Q_{p}}$}

This appendix proves the dependence of $z_{l_{p}}$ and $z_{Q_{p}}$ on each other. For simplicity and without loss of generality, the users superscripts $k$ have been dropped. The definitions of $z_{I_{p}}$ and $z_{Q_{p}}$ as given in equations (D.11) and (D.12) are repeated below for reference

$$
\begin{aligned}
& z_{I_{p}}=\frac{1}{T}\left[E_{p} \cos (\beta)-F_{p} \sin (\beta)\right] \\
& z_{Q_{p}}=\frac{1}{T}\left[F_{p} \cos (\beta)+E_{p} \sin (\beta)\right]
\end{aligned}
$$

The random variables $z_{p}$ and $z_{Q_{p}}$ are independent if and only if the product of their individual characteristic functions is equal to the joint characteristic function[120]

$$
\mathbf{E}\left\{\exp \left[\omega_{1} z_{l_{p}}+\omega_{2} z_{Q_{p}}\right]\right\}=\mathbf{E}\left\{\exp \left[\omega_{1} z_{p}\right]\right\} \cdot \mathbf{E}\left\{\exp \left[\omega_{2} z_{Q_{p}}\right]\right\}
$$

From equations (G.1)and (G.2), the lefthand side of equation (G.3) is given by (neglerting the factor of $1 / \mathrm{T}$ )

$$
\text { LIIS } \left.=\mathbf{E}\left\{\operatorname{xp}\left(\omega_{1} E_{p}+\omega_{2} F_{p}\right) \cos (\beta)+\left(\omega_{2} E_{p}-\omega_{1} F_{p}\right) \sin (\beta)\right]\right\}
$$




$$
\begin{gathered}
=\mathbf{E}\left\{\operatorname { e x p } \left(\sqrt{\left(\omega_{1} E_{p}+\omega_{2} F_{p}\right)^{2}+\left(\omega_{2} E_{p}-\omega_{1} F_{p}\right)^{2}} .\right.\right. \\
\left.\left.\cos \left(\beta-\tan ^{-1}\left(\frac{\omega_{2} E_{p}-\omega_{1} F_{p}}{\omega_{1} E_{p}+\omega_{2} F_{p}}\right)\right)\right)\right\} \\
=\mathbf{E}\left\{I_{0} \sqrt{\left(\omega_{1}^{2}+\omega_{2}^{2}\right) \cdot\left(\left(E_{p}\right)^{2}+\left(F_{p}\right)^{2}\right)}\right\}
\end{gathered}
$$

in which the last line of equation (G.4) results from taking the expectation with respect to $\beta$, a uniformly distributed random variable. Furthermore from the Taylor series expansion of $I_{0}$ as given in equation (B.7) the lefthand side of equation (G.4) can be written as

$$
\mathbf{E}\left\{\sum_{n=0}^{\infty} \frac{\left(\omega_{1}^{2}+\omega_{2}^{2}\right)^{n}\left(\left(E_{p}\right)^{2}+\left(F_{p}\right)^{2}\right)^{n}}{2^{2 n}(n !)^{2}}\right\}
$$

in which the expectation is now over $E_{p}$ and $F_{p}$.

Proceeding in a similar manner, the righthand side of equation (G.3) is given by

$$
\begin{aligned}
\text { RHS } & =\mathbf{E}\left\{I_{0}\left(\omega_{1} \sqrt{\left(E_{p}\right)^{2}+\left(F_{p}\right)^{2}}\right)\right\} \cdot \mathbf{E}\left\{I_{0}\left(\omega_{2} \sqrt{\left(E_{p}\right)^{2}+\left(F_{p}\right)^{2}}\right)\right\} \\
& =\mathbf{E}\left\{\sum_{n=0}^{\infty} \frac{\omega_{1}^{2 n}\left(\left(E_{p}\right)^{2}+\left(F_{p}\right)^{2}\right)^{n}}{2^{2 n}(n !)^{2}}\right\} \cdot \mathbf{E}\left\{\sum_{n=0}^{\infty} \frac{\omega_{2}^{2 n}\left(\left(E_{p}\right)^{2}+\left(F_{p}\right)^{2}\right)^{n}}{2^{2 n}(n !)^{2}}\right\}
\end{aligned}
$$

which is not equal to the lefthand side as given in equation (G:4). Thus $z_{p}$ and $z_{Q_{p}}$ are dependent. 


\section{References}

[1] M. Kavehrad and P. J. McLane, "Spread spectrum for indoor digital radio." IEEE Communication Magazine, vol. 25. no. 6, pp. 32-40, June 1987.

[2] M. Kavehrad. "Performance of nondiversity Receivers for spread spectrum in indoor wireless communications," ATET Technical Journal, vol. 64, no. 6, pp. 1181-1210, July-August 1985.

[3] M. Kavehrad and P. J. McLane, "Performance of low-complexity channel coding and diversity for spread spectrum in indoor wireless communication," ATET Technical Journal, vol. 64, no. B, pp. 1927-1965, October 1985.

[4] M. Kavehrad and B. Ramamurthi, "Direct sequence spread spectrum with DPSK modulation and diversity for indoor wireless communications," IEEE Transactions on Communications, vol. COM-35, ao. 2, pp. 224-236, February 1987.

[5] M. Kavehrad and G. E. Bodeep, "Design and experimental results for a direct sequence spread spectrum radio using differential phase shift keying modulatioul for indoor wireless communications," IEEE Journal on Selected A reas in Communication, vol. SAC-5, no. 5, pp. 815-825, June 1987.

[6] G. Ungerboeck, "Channel coding with multilevel phase signals," IEEE Transactions on Information Theory, vol. IT-28, no. 1, pp. 55-67, January 1982.

[i] A. R. Calderbank and N. J. A. Sloane, "New trellis codes based on lattices and cosets." IEEE Transactions on Information Theory, vol. IT-33, no. 2, pp. $17 i \cdot 195$, March 1987.

[8] R. J. C. Bultitude, “A comparison of indoor radio propagation characteristics at $910 \mathrm{MHz}$ and $1.75 \mathrm{GHz}, "$ IEEE Journal on Selected Areas in Co'"munications, vol. 7 , no. 1, pp. 20-30. January 1989. 
[9] D. J. Devasirvatham, "Time delay spread and signal level measurements of 850 $\mathrm{MHz}$ radio waves in building environments," IEEE Transactions on Antennas and Propagation, vol. AP-34, pp. 1300-1305, November 1986.

[10] G. L. Turis, "Introduction to spread spectrum anti-multipath techniques and their applications to urban digital radio," Proceedings of JEEE, vol. 68, no. 3 , pp. 328-353, March 1980.

[11] A. A. M. Saleh and R. A. Valenzuela, "A statistical model for indoor multipath propagation," IEEE Journal on Selected Areas in Communication, vol. SAC-5, no. 2, pp. 128-137, February 1987.

[12] M. B. Pursley, "Performance evaluation for phase-coded spread spertrum multiple acces conumunications - Part I: system analysis," IEEE Transactions on Communications, vol. COM-25, no. 8, pp. 795-799, August 1977.

[13] M. B. Pursley and D. V. Sarwate, "Performance evaluation for phase-coded spread spectrum multiple access communications - Part II: code sequence analysis," IEEE Trinsactions on Communications, vol. COM-25, no. 8, pp. 800-803, August 1977.

[14] M. B. Pursley, D. V. Sarwate and W. E. Stark, "Error probability for dircet sequence spread spectrum multiple access communirations -- Part I: upper and lower bounds," IEEE Transactions on Communications, vol. COM-30, no. 5, pp. 975-984, May 1982.

[15] M. B. Pursley, "Spread Spectrum Multiple-Access Communications," Multi-l/ser Communications, G. Longo Ed. Vienna and New York: Springer-Verlag, 1981, pp. 139-199.

[16] E. A. Geraniotis and M. B. Pursley, "Error probability for dirert srequence spread spectrum multiple access communications - Part II: approximations," IEEE Transactions on Communications, vol. COM-30, no. 5, pp. 985-995, May 1982.

[17] M. B. Pursley and F. D. Garber, "Quadriphase spread spectrum multiple access communications," In Conference Record 1978 International r'onference on Communications, pp. 7.3.1-7.3.5

[18] M. B.Pursley, F. D. Garber and J. S. Lehnert, "A nalysis of generalizod quadriphase spread spectrum communications," In Conference Record 1980 International Conference on Communications, pp. 15.3.1-15.3.6. 
[19] M. B. Pursley, “Spread Spectrum Multiple-Access Communications,” Multi-User Communications, G. Longo Ed. Vienna and New York: Springer-Verlag, 1981, pp. 139-199.

[20] D. V. Sarwate, M. B. Pursley and T. U. Basar, "Partial correlation effects in direct-sequence spread spectrum multiple-access communications systems," IEEE Transactions on Communications, vol. COM-32, no. 5, pp. 567-573, May 1984.

[21] K. Yao, "Error probability of asynchronous spread spectrus multiple access communication systems," IEEE Transactions on Communications, vol. COM25, no. 8, pp. 803-809, August 1977.

[22] D. Laforgia, A. Luvison, and V. Zingarelli, "Bit error rate evaluation for spread spectrum multiple access systems," IEEE Transactions on Communications. vol. COM-32, no. 6, pp. 660-667, June 1984.

[23] M. II. Meyers, "Computing the distribution of a random variable via Gaussian quadrature rules,” Bell System Technical Journal, vol. 61, no. 9, pF. 2245-2261, November 1982.

[24] M. Kavehrad and M. Joseph, "Maximum entropy and the method of moments in performance evaluation of digital communication systems," IEEE Transactions on Communications, vo. COM-34, no. 12, pp. 1183-1189, Dec. 1986.

[25] J. S. Lehnert and M. B, Pursley, "Error probability for binary direct sequence spread spectrum communications with random signature sequences," IEEE Transactions on Communications, vol. COM-35, no. 1, pp. 87-98, January 1987.

[26] J. E. Mazo, "Some theoretical observations on spread spectrum communications." Bell System Technical Journal, vol. 58, no. 9, pp. 2013-2023, November 1979.

[27] E. A. Geraniotis, "Direct sequence spread spectrum multiple access communications over nonselective and frequency selective Rician fading channels," IEEE Transactions on Communications, vol. COM-34, no. 8, pp. 756-764, August 1986.

[28] H. Ochsner. "Direct sequence spread spectrum receivers for communication on frequency-selective fading channels," IEEE Journal on Selected Areas in Communication. vol. SAC-5, no. 2, pp. 188-193, February 1987. 
[29] G. L. Turin, "The effects of multipath fading on the performance of direct sequence code division multiple access systems," IEEE Journal on Selerted A reas in Communication, vol. SAC-2, no. 4. pp. 597-603, July 1984.

[30] P. A. Bello, "Characterization of randomly time variant linear channels," IEEE Transactions on Communication Systems, pp. 360-393, December 1963.

[31] F. J. MacWilliams and N. J. A. Sloane, "Pseudo-random sequences and arrays," Proceedings of the IEEE, vol. 64, no. 12, pp. 1715-1730, December 1976.

[32] D. V. Sarwate and M. B. Pursley, "Cross correlation properties of pseudorandom and related sequences," Proceedings of the IEEE, vol. 68, no. 5, pp. 593-619, May 1980.

[33] A. J. Viterbi, "When not to spread spectrum - a sequel," IEEE ('ommuniration Society Magazine, vol. 23, no. 4, pp. 12-17, April 1985.

[34] F. J. MacWilliams and N. J. A. Sloane, The Theory of Error Correrting Codes, North-Holland Publishing Company, New York, 1977.

[35] W. W. Peterson and E. J. Weldon, Error Correcting Codes, MIT Press, Cambridge, Massachusetts, 1972.

[36] M. K. Simon, J. K. Omura, R. A. Scholtz and B. A. Levitt, Spread Spectrum Communications - Volume I, Computer Science Press, Maryland, 1985.

[37] L. R. Welch, "Lower bounds on the maximum cross-correlation of signals." IEEE Transactions on Information Theory, vol. IT-20, no. 3, pp. 397-399, Novernber 1979.

[38] D. V. Sarwate, "Bounds on crosscorrelation and autocorrelation of sequences," IEEE Transactions on Information Theory, vol. IT-25, no. 6, pp. 720-724, May 1984.

[39] V. M. Sidelnikov, -On mutual correlation of sequences," Sovit Math. Dokl., Vol. 12, no. 1, 1971.

[40] M. B. Pursley and H. F. A. Roefs, "Numerical evaluation of correlation parameters for optimal phases of binary shift register sequences," IEEE Transactions on Communications, vol. COM-27, no. 10, pp. 1597-1604. Ortuber 1979.

[41] H. E. Rowe, "Bounds on the number of signals with restricted cross correlation," IEEE Transactions on Communications, vol. COM-30, no. :5, pp. 96f;-974, May 1982. 
[42] R. Gold, "Optimal binary sequences for spread spectrum multiplexing," IEEE Transactions on Information Theory, vol. IT-13, no. 10, pp. 619-621, October 1967.

[43] R. Gold, "Maximal recursive sequences with 3-valued recursive cross-correlation functions," IEEE Transactions on Information Theory, vol. IT-14, no. 1, pp. 154-156, January 1968.

[44] R. C. Dixon, Spread Spectrum Systems, Second Edition, John Wiley and Sons, New York, 1984.

[45] T. Kasami, "Weight distribution of Bose-Chaudhuri-Hocquenghem codes," In Combinatorial Mathematics and Its Applications, Chapel Hill NC: University of North Carolina Press, 1969.

[16] R. I Blahut. Fast Algorithms for Digital Signal Processing, Addison-Wesley Publishi a Co.. New York. 1985.

[47] W. W. Wu. Elements of Digital Satellite Communication - Volume I, Computer Science Press, Maryland, 1984.

[48] J. D. Alanen, D. E. Knuth, "Tables of finite fields," Sankhyā, Series A, vol. 26, pp. 305-328. December 1964.

[49] T. Helleseth, "Some results about the cross-correlation function between two maximal linear sequences," Discrete Mathematics, Vol. 16, pp. 209-232, 1976.

[:00] P. A. N. Briggs and K. R. Godfrey, "Autocorrelation function of a 4-level Msequence." Electronics Lettrrs, vol. 4, pp. 232-233. May 1968.

[51] K. R. Godfrey, "Three level M-sequences," Electronics Letters, vol. 2, no. T, pp. 241-2.13, July 1966 .

[5:] D. H. Cireen and I. S. Taylor. "Irreducible polynomials over composite Galois Fields and their applicaicions in -oding techniques," Proceedings of the IEE, vol. 121, no. 9. pp. 935-939, September 1974.

[53] C. Balza. A. Frornageot and M. Maniere, "Four level pseudorandom sequences," Electronics Leiters, vol. 3, no. 7, pp. 313-315. July 1967.

[5.1] J. A. Chang. "Generation of 5-level maximum length sequences," Electrc rics Letters. vol. 2. pp. 258, 1966. 
[55] R. A. Scholtz and L. R. Welch, "Group characters: sequences with good corre lation properties," IEEE Transactions on Information Theory, vol. IT-2.1, no. 5, pp. 537-545, September 1978.

[56] R. Sivaswamy, "Multiphase complementary codes," IEEE Transactions on Information Theory, vol. IT-24, no. 5, pp. 546-552, September 1978.

[57] A. A. Shaar, C. F. Woodcock and P. A. Davies, "Bounds on the cross-correlation functions of state M-sequences," IEEE Transactions on Communications, vol. COM-35, no.3, pp. 305-312, March 1987.

[58] R. J. McEliece, "On periodic sequences from GF(q)," Journal of Combinatorial Theory, Vol. 10, pp. 80-91, 1971.

[59] M. B. Pursley and D. V. Sarwate, "Evaluation of correlation parameters for periodic sequences.” IEEE Transactions on Information Theory, vol. IT-23, no. 7, pp. 508-513, July 1977.

[60] G. Ungerboeck, "Trellis coded modulation with redundant signal sets: Part 1," IEEE Communications Magazine, vol. 25, no. 2, pp. 5-11, February 198 ?

[61] G. Ungerboeck, "Trellis coded modulation ..ith redundant signal sets: Part II," IEEE Communications Magazine, vol. 25, no. 2, pp. 12-21, February 1987.

[62] A. R. Calderbank and J. E. Mazo, "A new description of trellis codes," IEEE Transactions on Information Theory, vol. IT-30, no. 6, pp. 784-791, November 1984.

[63] G. D. Forney, "Coset codes I: Geometry and classification." IEEE Transartions on Information Theory, vol. I'T-34. no. 5. Part II pp. 1123-1151, September 1988.

[64] G. D. Forney, "Coset codes II: Binary lattices and relatcul codes," IFFE: Transactions on Information Theory, vol. IT -34. no. 5, Part II pp. 1152-1187, Septermber 1988 .

[65] L.-F. Wei, "Rotationally invariant convolutional channel coding with expander signal space - Part I - 180 ", IEEE Journal on Selected Areas in Communications, vol. SAC-2, no. 5, pp. 659-671, September 1984.

[66] L.-F. Wei, "Rotationally invariant convolutional channel coding with expandex] signal space - Part II: Nonlinear codes," IEEE Journal on Silterted A rras in Communications, vol. SAC-2, no. 5, pp. 672-686, Siptember 1984. 
[67] A. Gersho and V. B. Lawrence, "Multidimensional signal constellations for voiceband data transmission," IEEE Journal on Selected Areas in Communications, vol. SAC-2, no. 5, pp. 687-702, September 1984.

[68] A. R. Calderbank and N. J. A. Sloane, "Four dimensional modulation with an eight state trellis code," AT\&T Technical Journal, vol. 64, no. 5, pp. 1005-1017, May-June 1985.

[69] L. F. Wei, "Trellis-coded modulation with multidimensional constellations," IEEE Transactions on Information Theory, vol. IT-33, no. 4, pp. 483-501, July 1987.

[70] G. D. Forney, R. G. Gallager, G. R. Lang, F. M. Longstaff and S. U. Qureshi, "Efficient modulation for band-limited channels," IEEE Journal on Selected Areas in Communications, vol. SAC-2, no. 5, pp. 632-647, September 1984.

[71] E. L. Cusack. "Error control codes for QAM signalling," Electronics Letters. vol. 20. pp. 62-63, 1984.

[72] N. J. A. Sloane. 'Tables of sphere packings and spherical codes.' IEEE Transactions on Information Theory, vol. IT-27, no. 3, pp. 327-338, May 1981.

[i3] N. J. A. Sloane, -The packing of spheres," Scientific American, vol. 250, pp. 116-125, Januir-v 1984.

[74] J. Leech and N. J. .1. Sloane. "Sphere packings and error-correcting codes," Canadian Journal of Mathematics, vol. 23, no. 4, pp. 718-745, 1971.

[i5] E. S. Barnes and N. J. A. Sloane. "New Lattice Packings of Spheres." Canadian Journal of Matherratics. vol. 35, no. 1, pp. 117-130, 1983.

[76] J. Leech. "Notes on sphere packing," Canadian Journal of Mathematics, pp. $251-26 \pi, 1967$.

[7i] A. Bos. J. II. Conway and N. J. A. Sloane. "Further lattici packings in high dimensions," Ifathematika, vol. 29, part 2, pp. 171-180, December 1982.

[78] H. S. M. Coxeter. Regular Polytopes. Dover Publications Inc., New York. 1973.

[79] J. H. Conway and N. J. A. Sloane. "Fast quantizing and decoding algorithms for lattice quantizers and codes." IEEE Transactions on Infornation Theory, vol. IT.28, no. 2. pp. 227-232. March 1982.

[50] A. (iersio. -Asymptotically optimal block quantization." IEEE Transactions on Information Theory, vol. IT-25. no. 4, pp. 373-380. July 1979. 
[81] J. H. Conway and N. J. A. Sloane, "Voronoi regions of lattices, second moments of polytopes and quantization," IEEE Transactions on Information Theory, vol. IT-28, no. 2, pp. 211-226, March 1982.

[82] J. H. Conway and N. J. A. Sloane. "A fast encoding method for lattice codes and quantizers," IEEE Transactions on Information Theory, vol. IT -29, no. 6. pp. 820-825, November 1983.

[83] J. II. Conway and N. J. A. Sloane, "Soft decoding techniques for corles and lattices, including the Golay code and the Leech Lattice." IEEE Trnnsactions on Information Theory, vol. IT-32, no. 1, pp. 41-50, Jant ury 1986.

[84] A. R. Calderbank, J. E. Mazo and V. K. Wei. "Asymptotic upper bounds on the minimum distance of trellis codes," IEEE Transactions on Communirations. vol. COM-33, no. 4, pp. 305-309. April 1985.

[\$5] A. R. Calderbank, J. E. Mazo and II. M. Shapiro, "Upper bounds on the minimum distance of trellis codes," Bell System Technical Journal, vol. 62, no. X. pp. 2617-2646, October 1983.

[86] G. J. Pottie and D. P. Taylor, "Sphere-packing upper bounds on the free distance" of trellis codes." IEEE Transactions on Information Theory, vol. 34, no. 3, pp. 435-447, May 1988.

[87] E. Zehavi and J. K. IVolf, "On the performance evaluation of trellis codes," IEEE Transactions on Information Theory, vol. I'T-33, no. 2, pp. 196i-202, March 1987.

[88] D. Divsalar and M. K. Simon, "Trellis coding with assymetric modnlations," IEEE Transactions on Communications, vol. (COM-3.5. no. 2, pp. 131)-141, February 1987.

[89] E. Biglieri, "High level modulation and coding for nonlinear satellite channels," IEEE Transactions on Communications, vol. COM-32, no. 5, pp. 616-6i26, May 1984.

[90] J. K. Omura and M. K. Simon. "Modulation/Demodulation Lechniques for sated. lite communications - Part IV: Appendices," JPL Publication 8/-73, Pasadena. CA., November 1,1981.

[91] A. J. Viterbi and J. K. Omura. Principles of Digital Cornmunicat ton and Coding, McGraw-Ilill Book Co.. New York, 1979. 
[92] A. J. Viterbi, "Convolutional codes and their performances in communication systems," IEEE Transactions on Communication Technology, vol. COM-19, no.5, pp. 751-772, October 1971.

[9::] M. K. Simon and D. Divsalar, "Combined trellis coding with asymmetric MPSK modulation," JPL Publication 85-24, Pasaderia, CA., May 1,1985.

[94] S. V. Pizzi and S. G. Wilson, "Convolutional a " ${ }^{\prime}$ combined with continuous phase modulation," IEEE Transactions on Communications, vol. COM-3.3, no. 1, pp. 20-29, January 1985 .

[95] (i. J. Pottic and D. P. Taylor, "An approach to Ungerbeeck cocing for rectangular signal sets," IEEE Transactions on Information Theory, vol. IT-33, no. 2. pp. 285-289, March 1987.

[96] (i. J. Pottie and D. P. Taylor, “Multilevel codes based on partitioning," IEEE Transactions on Information Theory. vol. 35, no. 1. pp. 87-98. January 1989.

[9i] J. K. Wolf and G. Ungerboeck. "Trellis coding for partial response channels," IEEE Transactions on Communications, vol. COM-34, no. 8, pp. 765-773, August 1986.

98] M. Kavehrad and C.-E. WV. Sundberg, "Bit error probability of trellis-coded QAM over cross-coupled multidimensional channels," IEEE Transactions on Communications, vol. COM-35, no. 4. pp. 369-381, April 1987.

[99] ['. I'iret. "Bounds for codes over the unit circle." IEEE Transactions on Information Theory, vol. IT-32. no. 6, pp. 750-767, November 1986.

[100] li. Biglieri, "I "ngerloerk codes do not shape the signal power spectrum," IEEE Transactions on Information Theory, vol. IT-32, no. 4, pp. 595-596. July 1986.

[101] S. ( . Wilson. "Rate $5 / 6$ trellis-coded 8-PSK." IEEF Transactions on Commumecations, vol. (O.11-31. no. 10, pp. 1045-1048, October 1986.

[102] S. ( . Wilson, II. A. Sleeper. II, P. J. Scholt ter and M. T. Lyons, "Rate 3/4 convolutional coding of 16-PSK: Code desing and performance study," IEEE Transactions on Communications, ،ol. COM-32, no. 12, pp. 1308-1318, December 198.

[10:3] D. Divalar and M. K. Simon, "Multiple trellis coded modulation (MTCM)," IEIE: Tivensuctoms on Communications vol-36. no. 4. pp. 410-419. A pril 1988. 
[104] M. K. Simon and D. Divsalar. "The performance of trellis coded inultilevel DPSK on a fading mobile satellite channel," Cunference Recond 1987 Intermational Conference on Communications, pp. 21.2.1-21.2.7.

[105] S. G. Wilson and Y. S. Leung, "Trellis-coded phase modulation on Rayleigh channels," Conference Record 1987 International Conference on Communications, pp. 21.3.1-21.3.5.

[106] P. J. McLane, P. H. Wittke, P. K.-M. Ho and C. Loo. "PSK and DPSK trellis codes for fast fading shadowed mobile satellite communication channels," ('onference Recond 1987 International Conference on Communications, pp. 21.1.121.1.6.

[107] G. K. Huth, "Optimization of coded spread spectrum system performance," IEEE Transactions on Communications, vol. COM-25. 110. 8. p1). 763-7T0. August 1977.

[108] P. D. Shaft. "Low rat" convolutional code applications in spread spectrum communications," IEEE Transactions on Communirations, vol. ('OM-25, wo. 8, pp 815-821, August 1977 .

[109] J. M. Wozencraft and I. M. Jacobs, Principles of ("ommuntration Lingincernng. John Wiley and Sons, New York 1965.

[110] J. G. Proakis, Digital Communications, McGraw-Ilill Inc., New York, I9K3.

[111] A. J. Viterbi, "Error bounds for convolutional codes and an asymptotically optimum decoding algorithm," IEEE Transactions on Information Theory, vol. IT-13, no. 2, pp. $260-269$, A pril 1967.

[112] G. D. Forney, Jr., "The Viterbi algorithm," Prorcadings of Ihe H:l:E, vol. (i). no. 3, pp. 268-278. March 1973.

[113] G. D. Forney, Jr., "Convolutional codes: Algebraic structure," HEFE' Trunsartions on Information Theory, vol. IT-16, no. 6, pp. 720-73.

[114] D. Divsalar and M. K. Simon, "Trellis roded modulation for $1 \times()(1-9(j)(1)$ lit/s transmission over a fading mobile satellite channel," IEFE' T'mensartions on Journal on Selected Areas in Communications, vol. JSA( -35, , 11. 2, 11. I62. 175 , February 1987.

[115] D. Divsalar and M. K. Simon. "The design of trellis coded Mll'sk for farling, channels: Performance criteria," IEEE Transactions on Communaratones, vel. COM-36. no. 9. pp. 1004-1012. September 19x8. 
[16] D. Divsalar and M. K. Simon. "The design of trellis coded MPSK for fading channels: Set partitioning for optimum code design," IEEE Transactions on Communications, vol. COM-36, no. 9, pp. 1013-1021, September 1988.

[117] Handbook of Mathematical Functions with Formulas, Graphs and Mathematical Tables. Edited by M. Abramowitz and I. Stegun, U. S. Department of Commerce, National Bureau of Standards. Applied Mathematics Series-55. June 195.5 .

[118] J. A. Heller and I. M. Jacobs, "Convolutional Codes and Space Applications". IEEE Transactions on Communication Technology, vol. COM-19 no. 5, pp. 835848, October 1971.

[119] M. K. Simon and C. C. Wang, "Differential detection of gaussian MSK in a molile radio environment." IEEE Transactions on Vehicular Technology. vol. VT-33. no. 4. pp. 307-320, November 1984.

[120] A. Papoulis, Random l'ariables. and Stochastic Processes. McGraw-Ilill. New York 1965. 

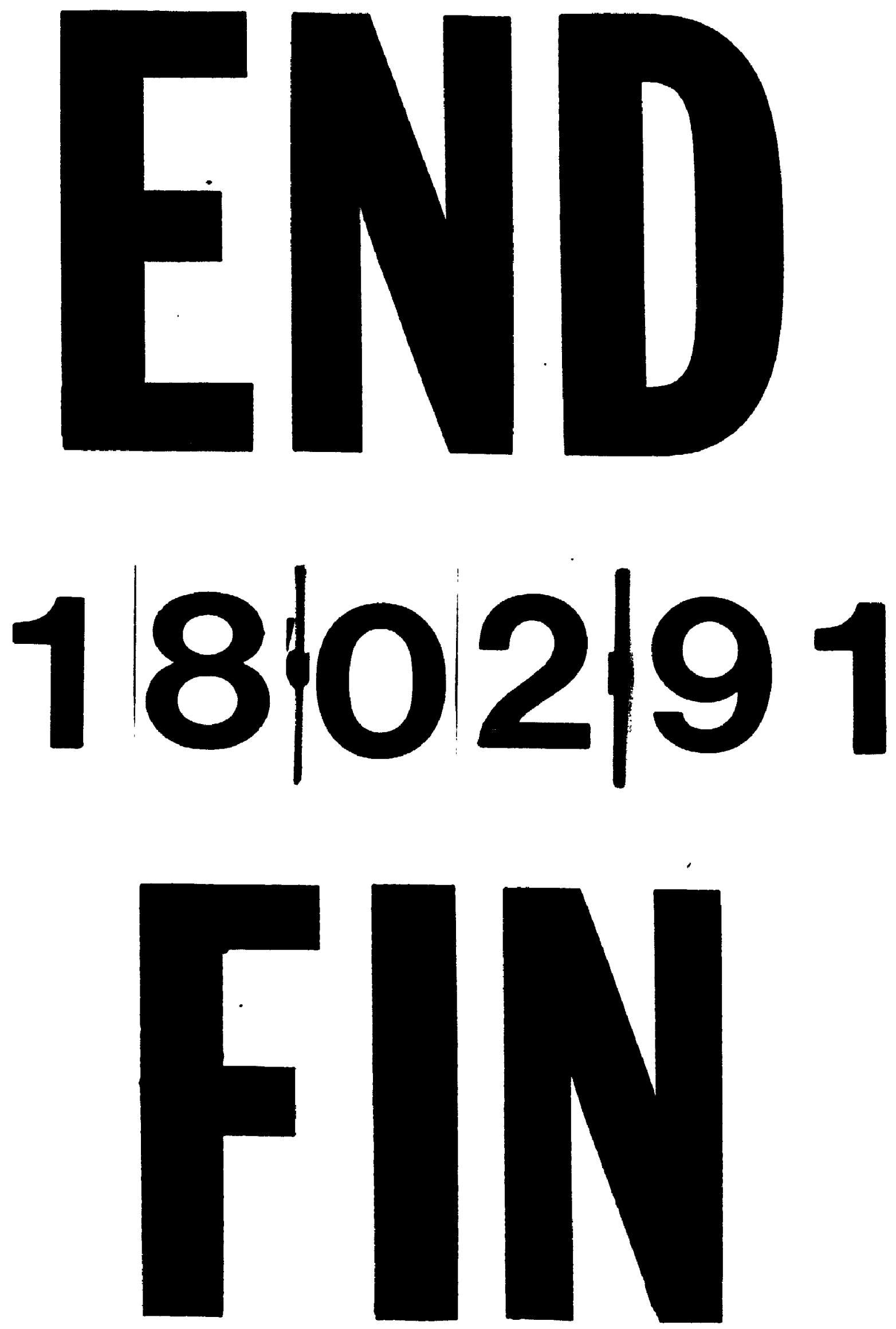